\title{
Asymmetric Hydrosilylation of the $\beta$-Silyl Styrenes Catalyzed by a Chiral Palladium Complex
}

\author{
Yi-Fan Wang, ${ }^{\mathrm{a}}$ Yu-Han He, ${ }^{\mathrm{b}}$ Yan Su, ${ }^{\mathrm{a}}$ Yang Ji, ${ }^{\mathrm{a}}$ and Rui Li*a \\ a State Key Laboratory of High-efficiency Coal Utilization and Green Chemical Engineering, \\ College of Chemistry and Chemical Engineering, Ningxia University, Yinchuan, 750021, China \\ b. College of Chemistry and Chemical Engineering, North Minzu University, Yinchuan 750021, \\ China. \\ Fax: +86(951)206-2323 \\ E-mail: ruili@nxu.edu.cn
}

\section{Supporting Information}

SI-1

SI-2

SI-3

SI-3

SI-4 to SI-6

SI-6

SI-7 to SI-31

SI-32 to SI-111
Table of Contents

General synthetic method for $\beta$-silyl styrenes, chiral $\beta$-hydroxy

silanes and 1,2-chiral bis(silyl) compounds

Synthetic method of racemic $\beta$-hydroxy silane

Determination of the absolute configuration of chiral $\beta$-hydroxy silane

DFT Study of intermediate hydridopalladium B

References

HPLC of chiral $\beta$-hydroxy silanes

Copies of NMR spectra for the key compounds 
Scheme SI-1. General synthetic method for $\beta$-silyl styrenes

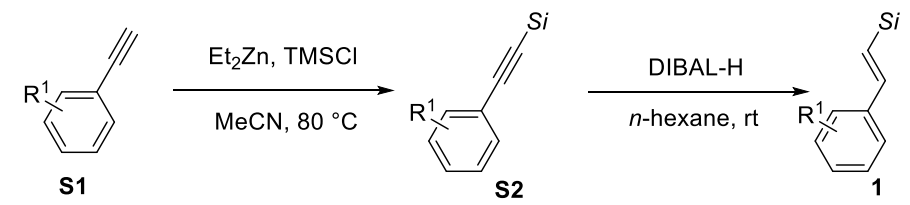

Scheme SI-2. General synthetic method for chiral $\beta$-hydroxy silanes

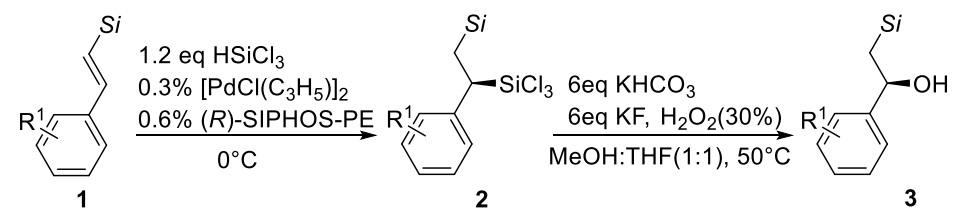

Scheme SI-3. General synthetic method for 1,2-chiral bis(silyl) compounds

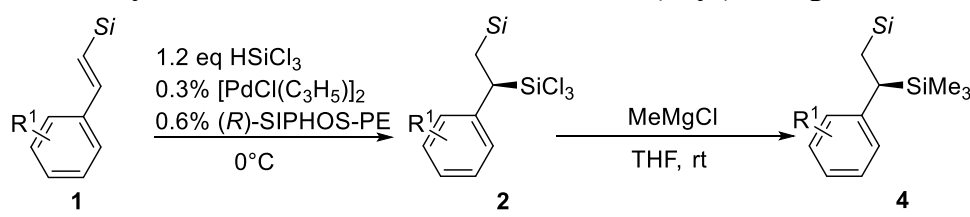

Scheme SI-4. Substrates of $\beta$-silyl styrenes

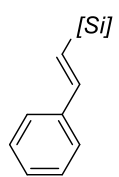

1a: $[\mathrm{Si}]=\mathrm{SiMe}_{3}$ 1b: $[\mathrm{Si}]=\mathrm{SiEt}_{3}$ 1c: $[S i]=\mathrm{SiMe}_{2} \mathrm{Ph}$ 1d: $[\mathrm{Si}]=\mathrm{SiMe}_{2}{ }^{{ }^{\mathrm{B}} \mathrm{Bu}}$ 1e: $[S i]=\mathrm{Si}^{i} \mathrm{Pr}_{3}$

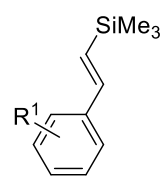

1f: $\mathrm{R}^{1}=2-\mathrm{Me}$ 1g: $\mathrm{R}^{1}=3-\mathrm{Me}$ 1h: $R^{1}=4-M e$ 1i: $R^{1}=4-{ }^{t} \mathrm{Bu}$ $1 \mathrm{j}: \mathrm{R}^{1}=4-\mathrm{OMe}$ 1k: $\mathrm{R}^{1}=2-\mathrm{C}$ 1I: $\mathrm{R}^{1}=3-\mathrm{Cl}$ $1 \mathrm{~m}: \mathrm{R}^{1}=4-\mathrm{Cl}$ 1n: $\mathrm{R}^{1}=4-\mathrm{Br}$ 10: $R^{1}=4-F$ 1p: $\mathrm{R}^{1}=4-\mathrm{CF}_{3}$

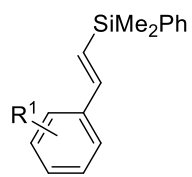

1q: $R^{1}=4-M e \quad 1 v: R^{1}=4-F$

1r: $R^{1}=4-{ }^{t} B u \quad 1 w: R^{1}=4-C_{3}$

1s: $\mathrm{R}^{1}=4-\mathrm{OMe}$

1t: $\mathrm{R}^{1}=4-\mathrm{Cl}$

1u: $\mathrm{R}^{1}=4-\mathrm{Br}$

Table SI-1. Yields of the $\beta$-silyl styrenes, chiral $\beta$-hydroxy silanes and 1,2-chiral bis(silyl) compounds

\begin{tabular}{|c|c|c|c|c|c|}
\hline $\mathbf{1}$ & Yield & $\mathbf{3}$ & Yield & $\mathbf{4}$ & Yield \\
\hline $\mathbf{1 a}$ & $1.6 \mathrm{~g}, 90 \%$ & $\mathbf{3 a}$ & $108.8 \mathrm{mg}, 56 \%$ & $\mathbf{4 a}$ & $227.9 \mathrm{mg}, 91 \%$ \\
\hline $\mathbf{1 b}$ & $3.1 \mathrm{~g}, 94 \%$ & $\mathbf{3 b}$ & $106.4 \mathrm{mg}, 45 \%$ & $\mathbf{4 b}$ & $275.0 \mathrm{mg}, 94 \%$ \\
\hline $\mathbf{1 c}$ & $1.9 \mathrm{~g}, 82 \%$ & $\mathbf{3 c}$ & $110.3 \mathrm{mg}, 43 \%$ & $\mathbf{4 c}$ & $300.1 \mathrm{mg}, 96 \%$ \\
\hline $\mathbf{1 d}$ & $1.7 \mathrm{~g}, 78 \%$ & $\mathbf{3 d}$ & $82.8 \mathrm{mg}, 35 \%$ & $\mathbf{4 d}$ & $272.1 \mathrm{mg}, 93 \%$ \\
\hline $\mathbf{1 e}$ & $2.0 \mathrm{~g}, 77 \%$ & $\mathbf{3 e}$ & $91.9 \mathrm{mg}, 33 \%$ & $\mathbf{4 e}$ & $287.8 \mathrm{mg}, 86 \%$ \\
\hline $\mathbf{1 f}$ & $1.6 \mathrm{~g}, 84 \%$ & $\mathbf{3 f}$ & $102.1 \mathrm{mg}, 49 \%$ & $\mathbf{4 f}$ & $246.1 \mathrm{mg}, 93 \%$ \\
\hline $\mathbf{1 g}$ & $1.5 \mathrm{~g}, 81 \%$ & $\mathbf{3 g}$ & $120.9 \mathrm{mg}, 58 \%$ & $\mathbf{4 g}$ & $238.1 \mathrm{mg}, 90 \%$ \\
\hline $\mathbf{1 h}$ & $1.7 \mathrm{~g}, 91 \%$ & $\mathbf{3 h}$ & $110.4 \mathrm{mg}, 53 \%$ & $\mathbf{4 h}$ & $240.8 \mathrm{mg}, 91 \%$ \\
\hline $\mathbf{1 i}$ & $1.7 \mathrm{~g}, 74 \%$ & $\mathbf{3 i}$ & $120.2 \mathrm{mg}, 48 \%$ & $\mathbf{4 i}$ & $275.9 \mathrm{mg}, 90 \%$ \\
\hline $\mathbf{1 j}$ & $1.5 \mathrm{~g}, 73 \%$ & $\mathbf{3 j}$ & $112.2 \mathrm{mg}, 50 \%$ & $\mathbf{4 j}$ & $260.9 \mathrm{mg}, 93 \%$ \\
\hline $\mathbf{1 k}$ & $1.6 \mathrm{~g}, 76 \%$ & $\mathbf{3 k}$ & $109.8 \mathrm{mg}, 48 \%$ & $\mathbf{4 k}$ & $256.5 \mathrm{mg}, 90 \%$ \\
\hline $\mathbf{1 I}$ & $1.6 \mathrm{~g}, 76 \%$ & $\mathbf{3 l}$ & $118.9 \mathrm{mg}, 52 \%$ & $\mathbf{4 l}$ & $259.3 \mathrm{mg}, 91 \%$ \\
\hline $\mathbf{1 m}$ & $1.5 \mathrm{~g}, 71 \%$ & $\mathbf{3 m}$ & $109.8 \mathrm{mg}, 48 \%$ & $\mathbf{4 m}$ & $259.3 \mathrm{mg}, 91 \%$ \\
\hline $\mathbf{1 n}$ & $1.8 \mathrm{~g}, 72 \%$ & $\mathbf{3 n}$ & $115.0 \mathrm{mg}, 42 \%$ & $\mathbf{4 n}$ & $296.5 \mathrm{mg}, 90 \%$ \\
\hline $\mathbf{1 0}$ & $2.4 \mathrm{~g}, 83 \%$ & $\mathbf{3 o}$ & $106.2 \mathrm{mg}, 50 \%$ & $\mathbf{4 0}$ & $244.4 \mathrm{mg}, 91 \%$ \\
\hline $\mathbf{1 p}$ & $1.7 \mathrm{~g}, 70 \%$ & $\mathbf{3 p}$ & $107.6 \mathrm{mg}, 41 \%$ & $\mathbf{4 p}$ & $286.7 \mathrm{mg}, 90 \%$ \\
\hline $\mathbf{1 q}$ & $1.7 \mathrm{~g}, 68 \%$ & $\mathbf{3 q}$ & $137.7 \mathrm{mg}, 52 \%$ & $\mathbf{4 q}$ & $310.3 \mathrm{mg}, 95 \%$ \\
\hline $\mathbf{1 r}$ & $1.6 \mathrm{~g}, 55 \%$ & $\mathbf{3 r}$ & $175.0 \mathrm{mg}, 56 \%$ & $\mathbf{4 r}$ & $331.8 \mathrm{mg}, 90 \%$ \\
\hline $\mathbf{1 s}$ & $1.7 \mathrm{~g}, 63 \%$ & $\mathbf{3 s}$ & $170.7 \mathrm{mg}, 60 \%$ & $\mathbf{4 s}$ & $304.9 \mathrm{mg}, 89 \%$ \\
\hline $\mathbf{1 t}$ & $1.7 \mathrm{~g}, 63 \%$ & $\mathbf{3 t}$ & $125.1 \mathrm{mg}, 43 \%$ & $\mathbf{4 t}$ & $312.3 \mathrm{mg}, 90 \%$ \\
\hline
\end{tabular}




\begin{tabular}{|c|c|c|c|c|c|}
\hline $\mathbf{1 u}$ & $1.9 \mathrm{~g}, 61 \%$ & $\mathbf{3 u}$ & $147.5 \mathrm{mg}, 44 \%$ & $\mathbf{4 u}$ & $364.1 \mathrm{mg}, 93 \%$ \\
\hline $\mathbf{1 v}$ & $1.7 \mathrm{~g}, 70 \%$ & $\mathbf{3 v}$ & $131.7 \mathrm{mg}, 48 \%$ & $\mathbf{4 v}$ & $297.5 \mathrm{mg}, 90 \%$ \\
\hline $\mathbf{1 w}$ & $1.7 \mathrm{~g}, 57 \%$ & $\mathbf{3 w}$ & $147.9 \mathrm{mg}, 48 \%$ & $\mathbf{4 w}$ & $338.7 \mathrm{mg}, 89 \%$ \\
\hline
\end{tabular}

Figure SI-1. Additional experimental details, materials, and methods, including photographs of experimental setup

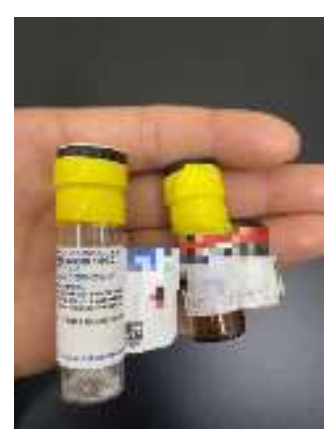

Picture 1 SIPHOS and Palladium

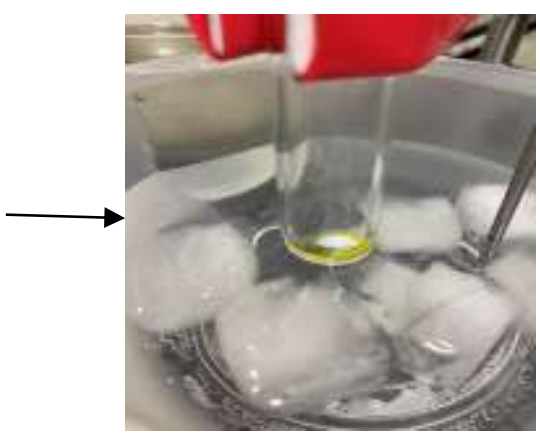

Picture 2 Initial system state of reaction

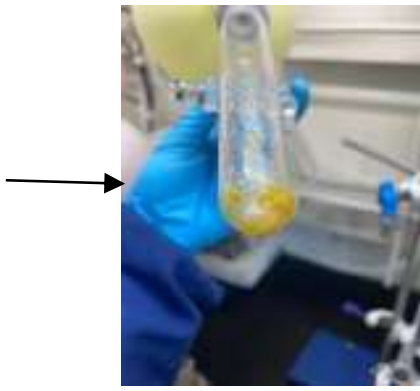

Picture 3 Product of 2a

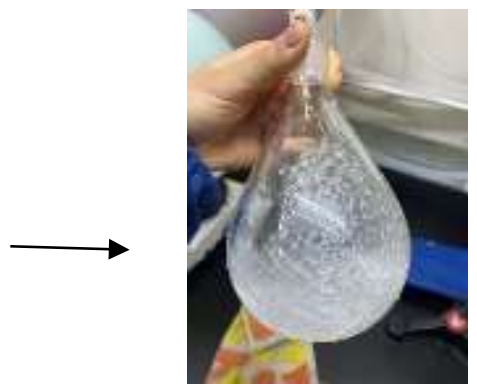

Picture 4 Product of $\mathbf{3 a}$

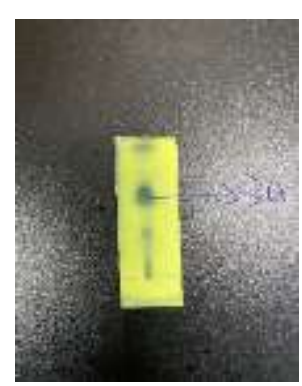

Picture 5 TLC of Tamao-Fleming reaction

Scheme SI-5. Synthesis of racemic $\beta$-hydroxy silane

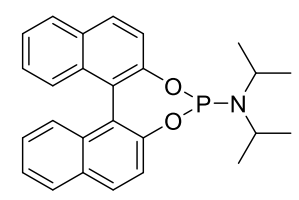

The title compound was prepared according to a literature procedure ${ }^{1}$.

The racemic phenylsilanols were prepared as described the procedure for asymmetric hydrosilylation of ß-silyl stryenes with trichlorosilane.

Scheme SI-6. Determination of the absolute configuration of chiral $\beta$-hydroxy silane

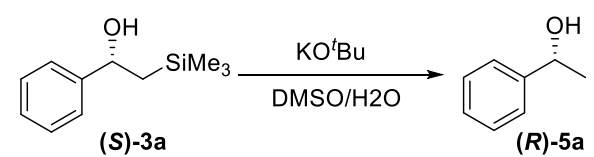

Following the procedure of Ortiz and coworks ${ }^{2}$, a $50 \mathrm{~mL}$ Round-bottomed flask was charged with $5 \%$ solution of potassium tert-butoxide in 19:1 (DMSO/ $\left.\mathrm{H}_{2} \mathrm{O}\right)$ and $261.9 \mathrm{mg}(1.35 \mathrm{mmol})$ of 3a. The reaction mixture was stirred at room temperature for $12 \mathrm{~h}$, water was added and the mixture was 
extracted with ether $(3 \times 20 \mathrm{~mL})$.The combined organic extracts were washed with water and dried over $\mathrm{Na}_{2} \mathrm{SO}_{4}$. The sovlents were removed at reduced pressure. Filtration of the crude over silica gel chromatography with EtOAc/PE $=1 / 30$ gave $98 \mathrm{mg}(60 \%)$ of the $5 \mathbf{a} .{ }^{1} \mathbf{H} \mathbf{~ N M R}\left(\mathrm{CDCl}_{3}, 400 \mathrm{MHz}\right)$ $\delta 7.29-7.42(\mathrm{~m}, 5 \mathrm{H}), 4.91(\mathrm{q}, J=6.4 \mathrm{~Hz}, 1 \mathrm{H}), 2.02(\mathrm{~s}, 1 \mathrm{H}), 1.52(\mathrm{~d}, J=6.4 \mathrm{~Hz}, 3 \mathrm{H}) .{ }^{13} \mathbf{C}$ NMR $\left\{{ }^{1} \mathrm{H}\right\}\left(\mathrm{CDCl}_{3}, 100 \mathrm{MHz}\right.$, plus, APT) $\delta$ (up) 146.0. $\delta$ (down) 128.5, 127.5, 125.4, 70.8, 25.2. The absolute configuration at the stereogenic center of $\mathbf{3 a}$ was assigned as $(S)$ - configuration $\mathrm{S}$ by desilylation of the sample into the known $(R)-1$-phenylethan-1-ol ${ }^{3}$.

\section{DFT Study}

\section{Details of DFT calculations}

We have performed a DFT mechanistic study using Gaussian 09. The structures of intermediate B were optimized at the B3LYP/BSI level in the gas phase, where BSI denotes a basis set 6-311G (d, p) for atoms H, C, N, O, P, N, Cl and Si, SDD for atom Pd.

Figure SI-2. Enantioselective model of Asymmetric Hydrosilylation of the ß-Silyl Styrenes

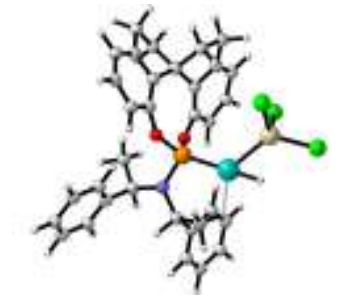

(A) Optimal structures of the hydrido(silyl)-palladium B

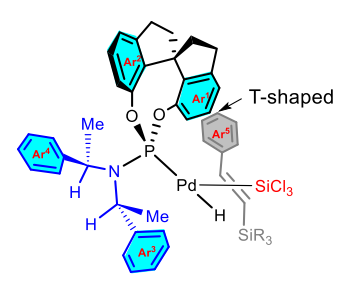

(B) proposed stereochemical model for the hydrosilylation reaction

Hydrido(silyl)-palladium B

\begin{tabular}{llll}
\hline $\mathrm{P}$ & 0.57225500 & 0.05373500 & -0.09115200 \\
$\mathrm{O}$ & 0.51057300 & 1.59324500 & -0.57747600 \\
$\mathrm{O}$ & 0.07869400 & 0.18044100 & 1.42365800 \\
$\mathrm{~N}$ & 2.23644200 & -0.15968100 & 0.05236700 \\
$\mathrm{C}$ & 2.89445500 & -1.41597300 & -0.41825400 \\
$\mathrm{C}$ & 3.02473400 & -2.46664400 & 0.68942800 \\
$\mathrm{C}$ & 4.23451800 & -3.14463600 & 0.89193000 \\
$\mathrm{C}$ & 1.92252900 & -2.81452400 & 1.48189900 \\
$\mathrm{C}$ & 4.34539100 & -4.14273000 & 1.86395900 \\
$\mathrm{H}$ & 5.09684100 & -2.89795700 & 0.27640600 \\
$\mathrm{C}$ & 2.02862200 & -3.81291900 & 2.44927800 \\
$\mathrm{H}$ & 0.97093800 & -2.30838000 & 1.33785600 \\
$\mathrm{C}$ & 3.24031800 & -4.48023800 & 2.64502200 \\
$\mathrm{H}$ & 5.29197200 & -4.65932800 & 2.00374100 \\
$\mathrm{H}$ & 1.16163600 & -4.07443800 & 3.05138800 \\
$\mathrm{H}$ & 3.31803100 & -5.26126400 & 3.39729800 \\
$\mathrm{C}$ & 2.30813800 & -2.11497000 & -1.66784700
\end{tabular}




\begin{tabular}{|c|c|c|c|}
\hline $\mathrm{H}$ & 3.03780400 & -2.82654200 & -2.06760800 \\
\hline $\mathrm{H}$ & 1.46023200 & -2.74643400 & -1.26461700 \\
\hline $\mathrm{H}$ & 2.03535800 & -1.45013200 & -2.50261200 \\
\hline $\mathrm{H}$ & 3.89950000 & -1.10528100 & -0.72303200 \\
\hline $\mathrm{C}$ & 3.16974900 & 0.71029500 & 0.86200200 \\
\hline $\mathrm{H}$ & 3.74913100 & 0.01720700 & 1.49422300 \\
\hline $\mathrm{C}$ & 4.15629000 & 1.46630400 & -0.02153300 \\
\hline $\mathrm{C}$ & 5.52654100 & 1.41036800 & 0.27104800 \\
\hline $\mathrm{C}$ & 3.73860500 & 2.25983500 & -1.09910500 \\
\hline $\mathrm{C}$ & 6.45735600 & 2.11794600 & -0.49403900 \\
\hline $\mathrm{H}$ & 5.86916300 & 0.80796000 & 1.11055900 \\
\hline $\mathrm{C}$ & 4.66579500 & 2.95413000 & -1.87501600 \\
\hline $\mathrm{H}$ & 2.68125900 & 2.32533600 & -1.33691800 \\
\hline $\mathrm{C}$ & 6.02896200 & 2.88693900 & -1.57598300 \\
\hline $\mathrm{H}$ & 7.51493300 & 2.05744600 & -0.24927200 \\
\hline $\mathrm{H}$ & 4.32223400 & 3.55535100 & -2.71368200 \\
\hline $\mathrm{H}$ & 6.74838500 & 3.43330100 & -2.18114300 \\
\hline $\mathrm{C}$ & 2.46300000 & 1.66604200 & 1.83474600 \\
\hline $\mathrm{H}$ & 1.94434700 & 1.12523400 & 2.63366200 \\
\hline $\mathrm{H}$ & 3.23471900 & 2.29430200 & 2.29542100 \\
\hline $\mathrm{H}$ & 1.75135200 & 2.32052400 & 1.32738700 \\
\hline $\mathrm{C}$ & -0.97850200 & 0.31573800 & 2.25366600 \\
\hline $\mathrm{C}$ & -2.01913600 & 1.17513100 & 1.92974800 \\
\hline $\mathrm{C}$ & -1.05379700 & -0.54732200 & 3.35628700 \\
\hline $\mathrm{C}$ & -3.27869600 & 0.87810800 & 2.49868400 \\
\hline $\mathrm{C}$ & -2.25902100 & -0.67469400 & 4.03514400 \\
\hline $\mathrm{H}$ & -0.18894700 & -1.16066000 & 3.59003400 \\
\hline $\mathrm{C}$ & -3.40786300 & -0.01284000 & 3.55607100 \\
\hline $\mathrm{H}$ & -2.33326200 & -1.36168600 & 4.87415200 \\
\hline $\mathrm{H}$ & -4.38504200 & -0.22928800 & 3.98061400 \\
\hline $\mathrm{C}$ & -0.30539500 & 2.65651400 & -0.86450600 \\
\hline $\mathrm{C}$ & 0.06266400 & 3.41624100 & -1.98823600 \\
\hline $\mathrm{C}$ & -1.29959400 & 3.11002200 & 0.01676800 \\
\hline $\mathrm{C}$ & -0.45777200 & 4.69506700 & -2.16103400 \\
\hline $\mathrm{H}$ & 0.79548200 & 3.00137800 & -2.67335100 \\
\hline $\mathrm{C}$ & -1.69148000 & 4.46333400 & -0.10129900 \\
\hline $\mathrm{C}$ & -1.29873700 & 5.24697700 & -1.18024900 \\
\hline $\mathrm{H}$ & -0.16239600 & 5.28444100 & -3.02545900 \\
\hline $\mathrm{H}$ & -1.62712100 & 6.28102200 & -1.25863400 \\
\hline $\mathrm{C}$ & -2.17954500 & 2.46975800 & 1.11100300 \\
\hline $\mathrm{C}$ & -4.38253100 & 1.54070900 & 1.69027400 \\
\hline $\mathrm{H}$ & -5.11191200 & 0.79954100 & 1.34318100 \\
\hline $\mathrm{H}$ & -4.94314300 & 2.28403800 & 2.27563000 \\
\hline $\mathrm{C}$ & -2.50194200 & 4.88626300 & 1.10770500 \\
\hline
\end{tabular}




$\begin{array}{llll}\mathrm{H} & -3.56723400 & 5.00351700 & 0.85360000 \\ \mathrm{H} & -2.16117100 & 5.84771700 & 1.51064700 \\ \mathrm{C} & -2.27676500 & 3.71123300 & 2.07803100 \\ \mathrm{H} & -1.31342800 & 3.82804100 & 2.58971700 \\ \mathrm{C} & -3.61752400 & 2.20519700 & 0.52542400 \\ \mathrm{Pd} & -0.25184900 & -1.67740700 & -1.49715100 \\ \mathrm{H} & -0.81108500 & -2.85215700 & -2.45126000 \\ \mathrm{Si} & -2.53040100 & -2.17055700 & -1.55859600 \\ \mathrm{Cl} & -3.31687500 & -2.05302400 & 0.39208000 \\ \mathrm{Cl} & -3.37149300 & -3.99102500 & -2.18594300 \\ \mathrm{Cl} & -3.49463300 & -0.75521100 & -2.76284400 \\ \mathrm{H} & -3.51814900 & 1.50555300 & -0.31397000 \\ \mathrm{H} & -4.10025600 & 3.11301100 & 0.14606900 \\ \mathrm{H} & -3.04775900 & 3.61884800 & 2.84846000\end{array}$

\section{References:}

(1) Ma, X. F. I.; Hazelden, R.; Langer, T.; Munday, R. H.; J. F. Bower. Enantioselective aza-heck cyclizations of N-(Tosyloxy)carbamates: synthesis of pyrrolidines and piperidines. J. Am. Chem. Soc. 2019, 141, 3356.

(2) Larson, G. L.; Prieto, J. A.; Ortiz, E. The preparation and chemistry of (S)-(1-naphthyl) phenylmethylsilylmethyllithium: stereochemistry at silicon in the elimination of $\beta$ hydroxysilanes, Tetrahedron., 1988, 44, 3781.

(3) Jensen, J. F.; Svendsen, B. Y.; Cour, T. V.; Pedersen, H. L.; Johannsen, M. Highly enantioselective hydrosilylation of aromatic alkenes. J. Am. Chem. Soc. 2002, 124, 4558. 


\section{HPLC of $\beta$-hydroxy silane}<smiles>CCCC(O)c1ccccc1</smiles>

Racemic sample:

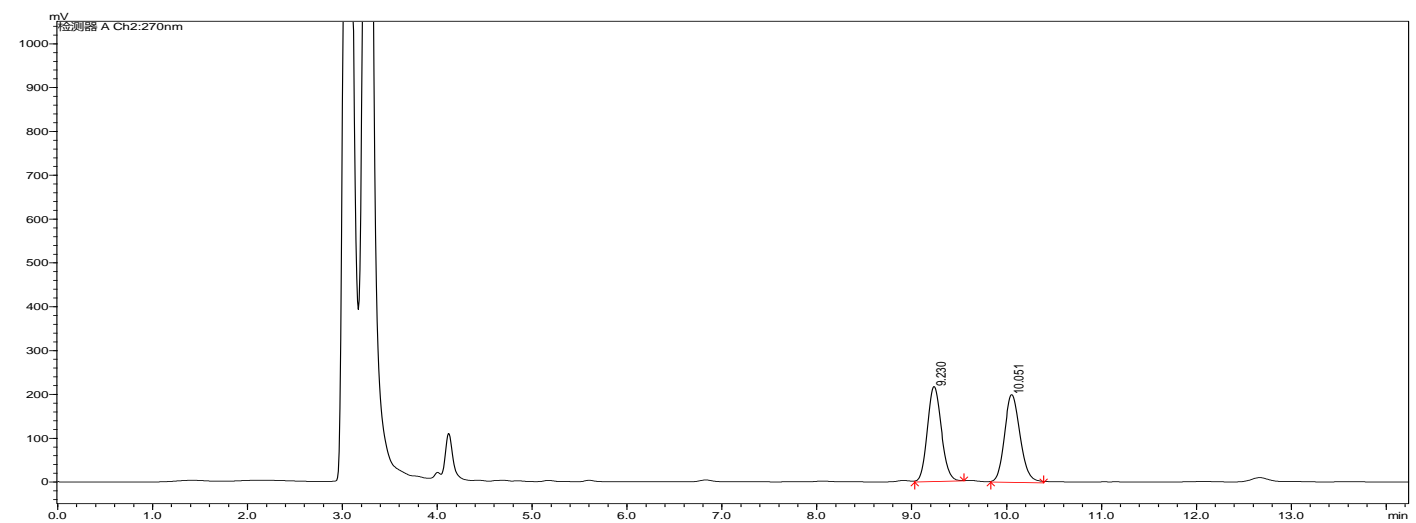

\begin{tabular}{|l|l|l|l|}
\hline Time & Conc. & Area & Height \\
\hline 9.230 & 49.70849 & 2227364 & 216993 \\
\hline 10.051 & 50.25631 & 2300026 & 199720 \\
\hline
\end{tabular}

Enantio-enriched sample:

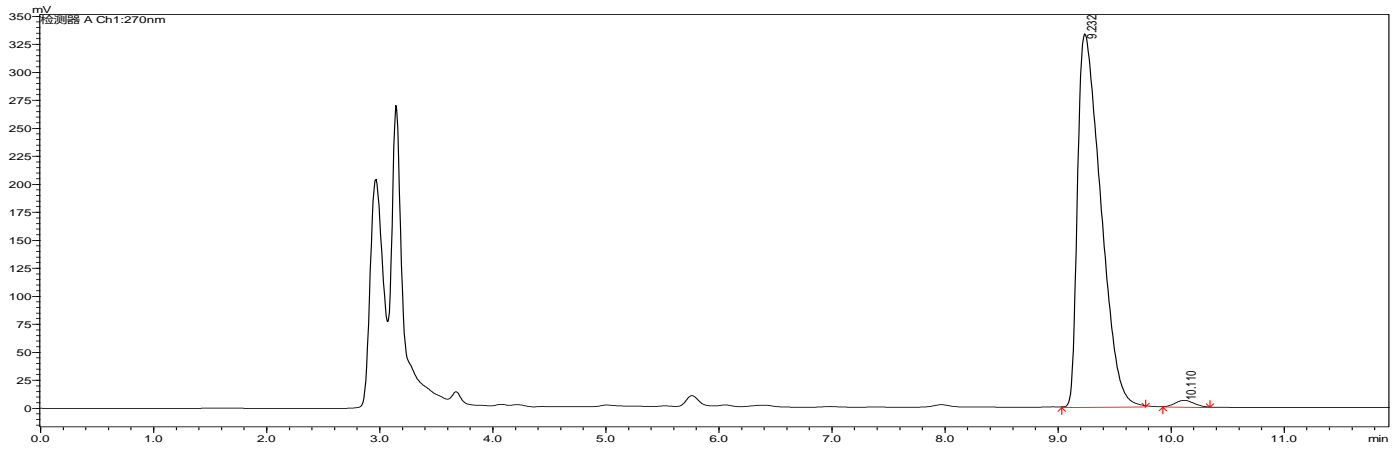

\begin{tabular}{|l|l|l|l|}
\hline Time & Conc. & Area & Height \\
\hline 9.232 & 98.85748 & 6560953 & 333713 \\
\hline 10.110 & 1.04252 & 74589 & 6175 \\
\hline
\end{tabular}

$98 \%$ ee

Column: AD-H;

Eluent: $n$-Hexane: $i$-PrOH = 98:2;

Flow rate: $1 \mathrm{~mL} / \mathrm{min}$;

Pressure: $3.8 \mathrm{Mpa}$;

Wavelength: $260 \mathrm{~nm}$;

Temperature: $35^{\circ} \mathrm{C}$ 


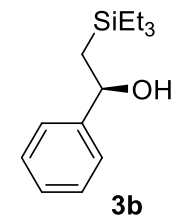

Racemic sample:

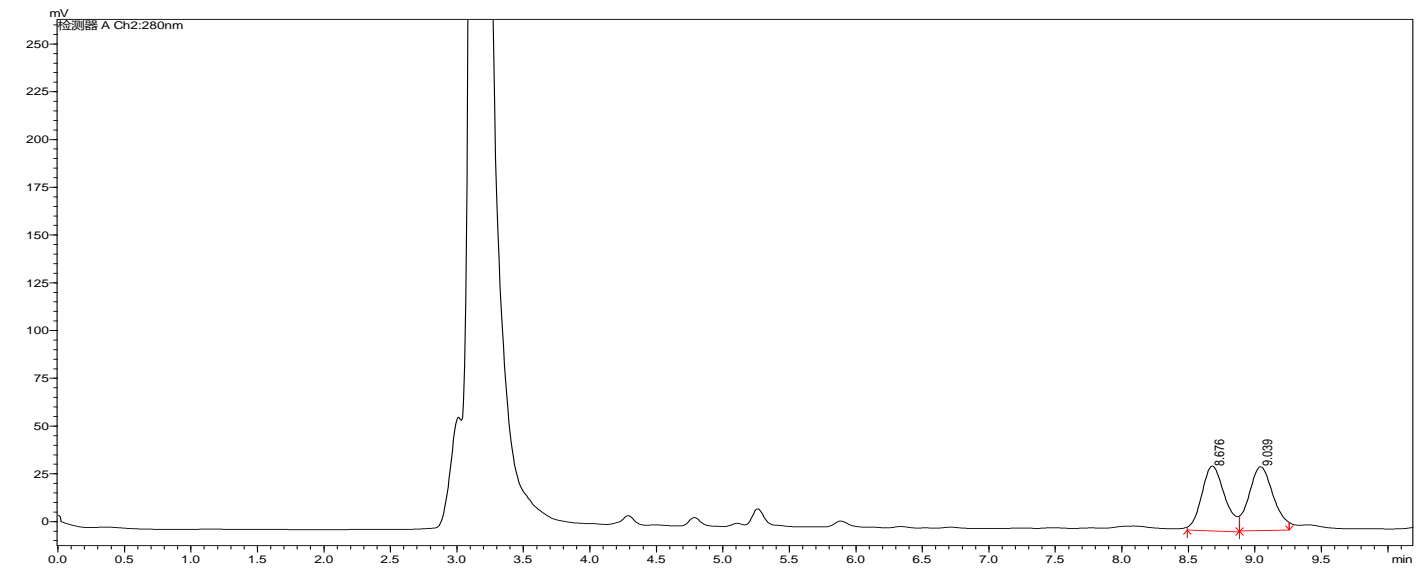

\begin{tabular}{|l|l|l|l|}
\hline Time & Conc. & Area & Height \\
\hline 8.676 & 49.71407 & 415441 & 33887 \\
\hline 9.039 & 50.28593 & 420220 & 33410 \\
\hline
\end{tabular}

Enantio-enriched sample:

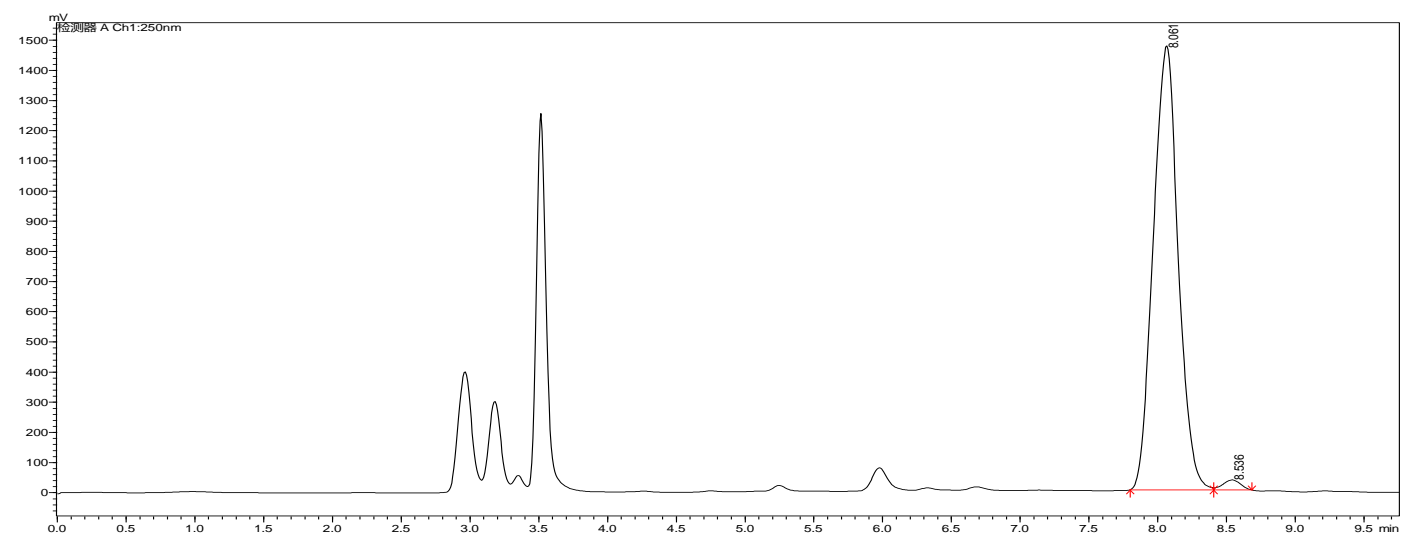

\begin{tabular}{|l|l|l|l|}
\hline Time & Conc. & Area & Height \\
\hline 8.061 & 98.44851 & 18566950 & 1472357 \\
\hline 8.536 & 1.55149 & 292603 & 33217 \\
\hline
\end{tabular}

Column: AD-H;

Eluent: $n$-Hexane: $i-\mathrm{PrOH}=98: 2$;

Flow rate: $1 \mathrm{~mL} / \mathrm{min}$;

Pressure: 3.9 Mpa;

Wavelength: $260 \mathrm{~nm}$;

Temperature: $35^{\circ} \mathrm{C}$ 


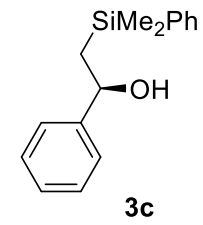

Racemic sample:

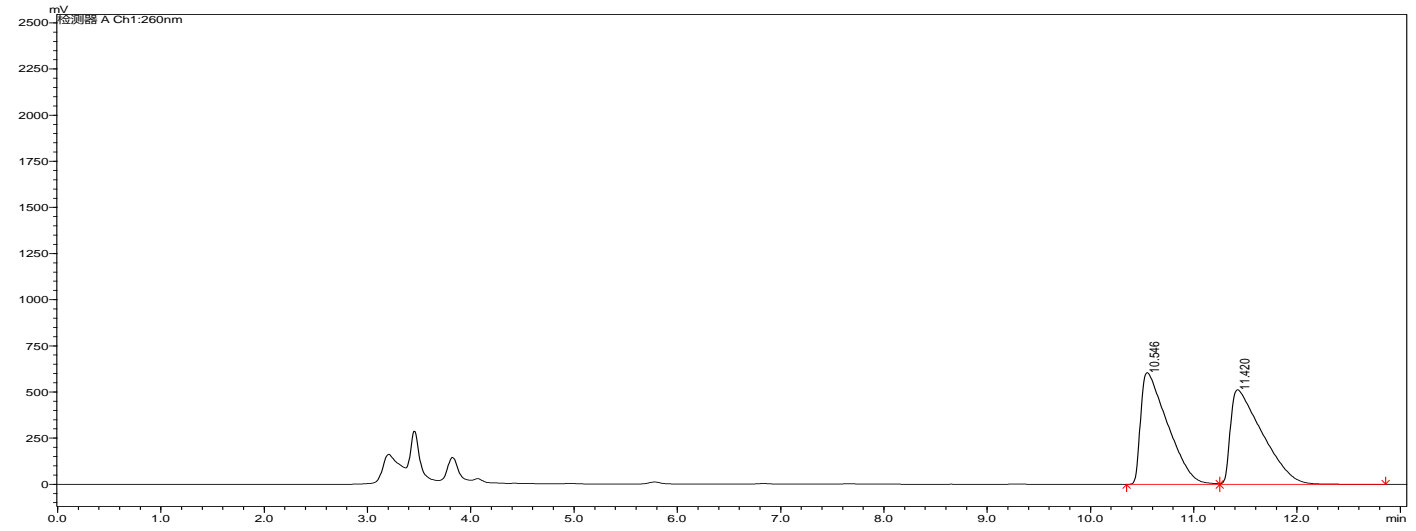

\begin{tabular}{|l|l|l|l|}
\hline Time & Conc. & Area & Height \\
\hline 10.546 & 49.95729 & 10952008 & 605327 \\
\hline 11.420 & 50.04271 & 10970733 & 512740 \\
\hline
\end{tabular}

Enantio-enriched sample:

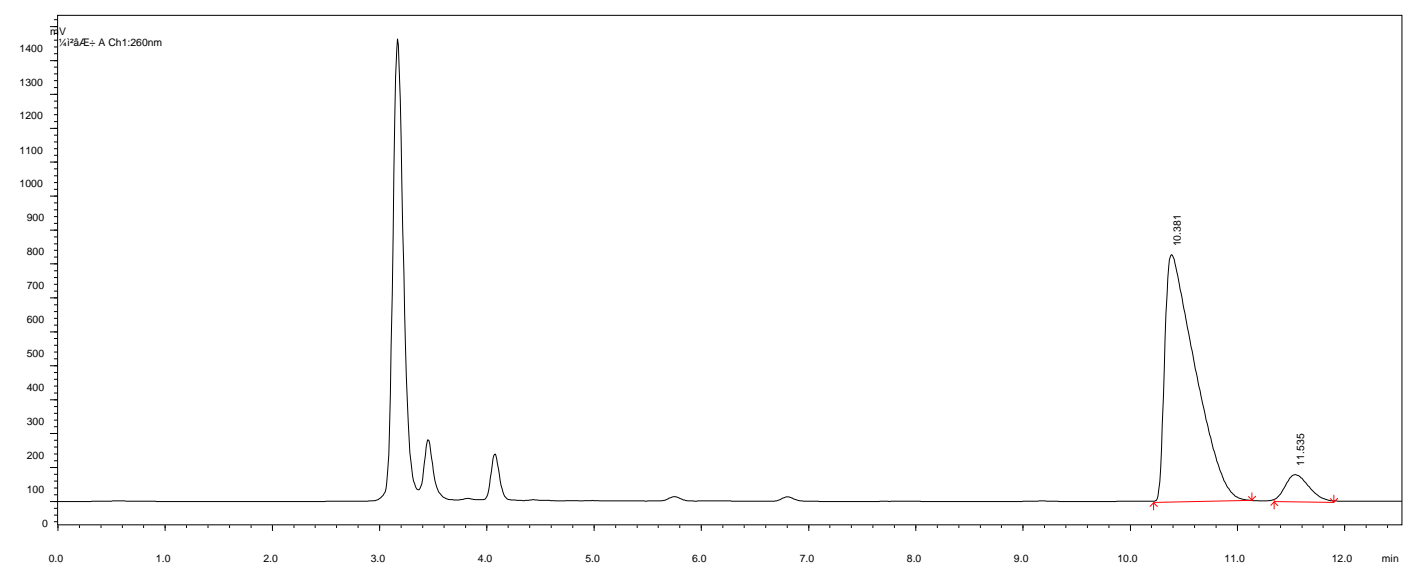

\begin{tabular}{|l|l|l|l|}
\hline Time & Conc. & Area & Height \\
\hline 10.381 & 91.91851 & 14372785 & 729566 \\
\hline 11.535 & 8.08149 & 1263657 & 79726 \\
\hline
\end{tabular}

$84 \%$ ee

Column: AD-H;

Eluent: $n$-Hexane: $i$-PrOH $=98: 2$;

Flow rate: $1 \mathrm{~mL} / \mathrm{min}$;

Pressure: $3.7 \mathrm{Mpa}$;

Wavelength: $260 \mathrm{~nm}$;

Temperature: $35^{\circ} \mathrm{C}$ 


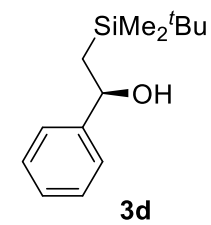

Racemic sample:

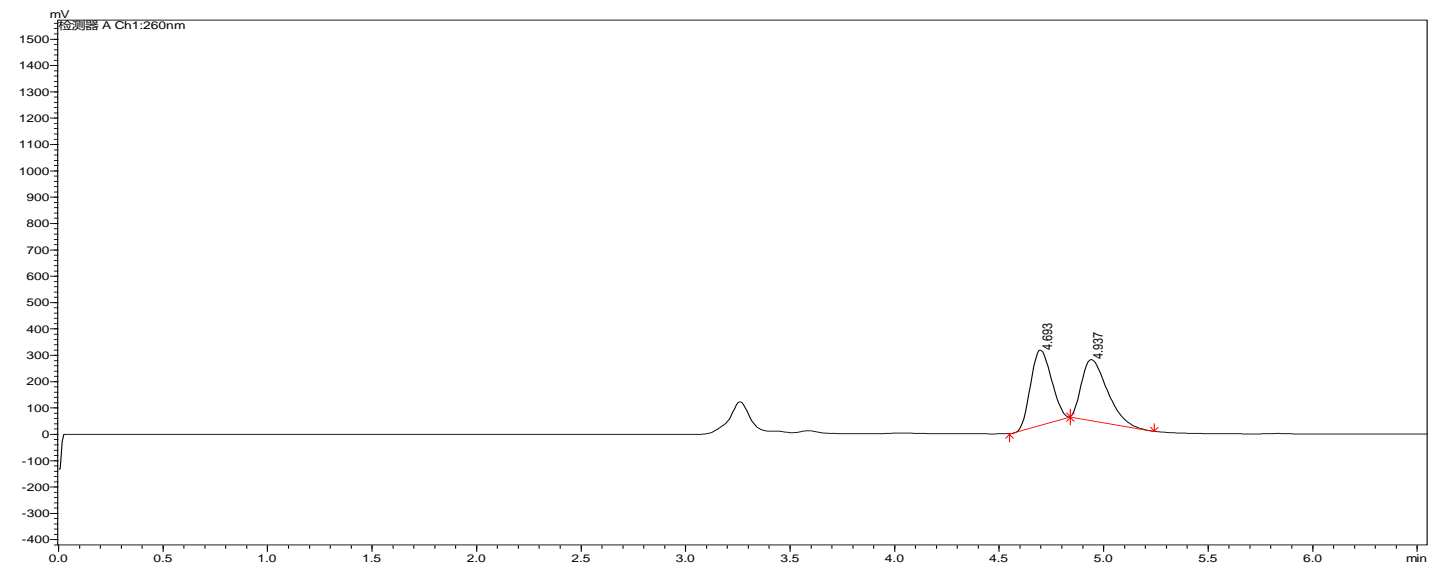

\begin{tabular}{|l|l|l|l|}
\hline Time & Conc. & Area & Height \\
\hline 4.695 & 50.32513 & 2002205 & 287427 \\
\hline 4.939 & 49.67487 & 1976334 & 231378 \\
\hline
\end{tabular}

Enantio-enriched sample:

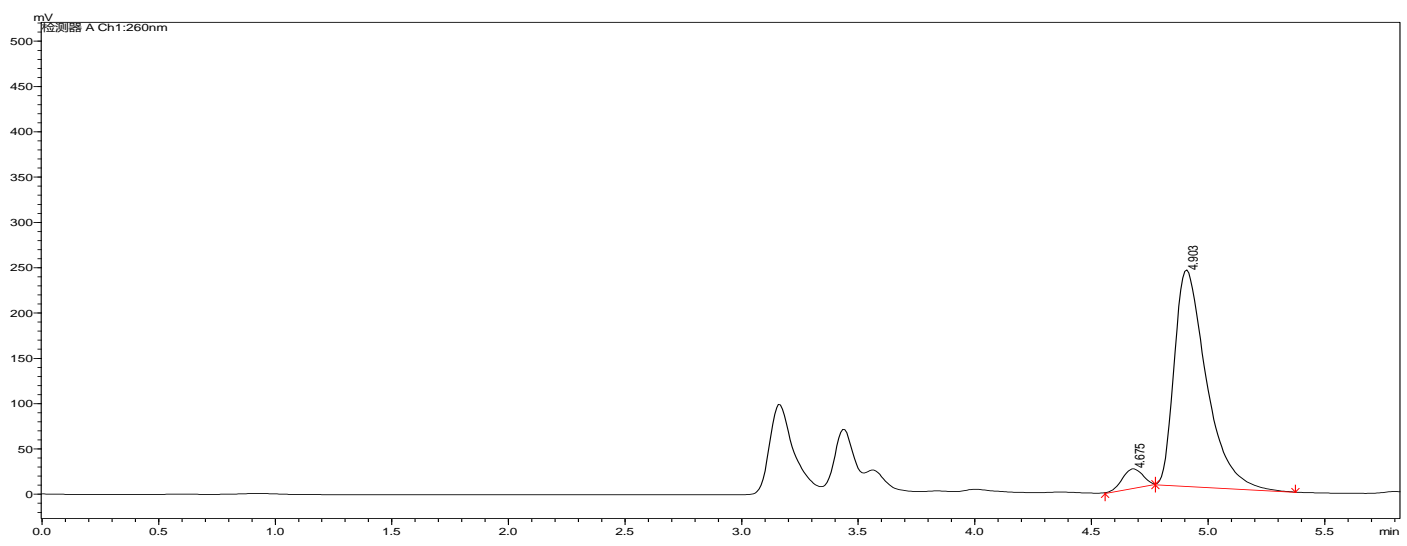

\begin{tabular}{|l|l|l|l|}
\hline Time & Conc. & Area & Height \\
\hline 4.675 & 5.64226 & 136761 & 21717 \\
\hline 4.903 & 94.35784 & 2287138 & 238911 \\
\hline
\end{tabular}

$89 \%$ ee

Column: AY-H;

Eluent: $n$-Hexane: $i$-PrOH $=98: 2$;

Flow rate: $1 \mathrm{~mL} / \mathrm{min}$;

Pressure: $3.7 \mathrm{Mpa}$;

Wavelength: $260 \mathrm{~nm}$;

Temperature: $35^{\circ} \mathrm{C}$ 


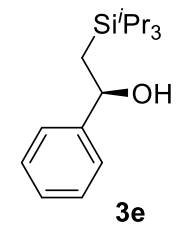

Racemic sample:

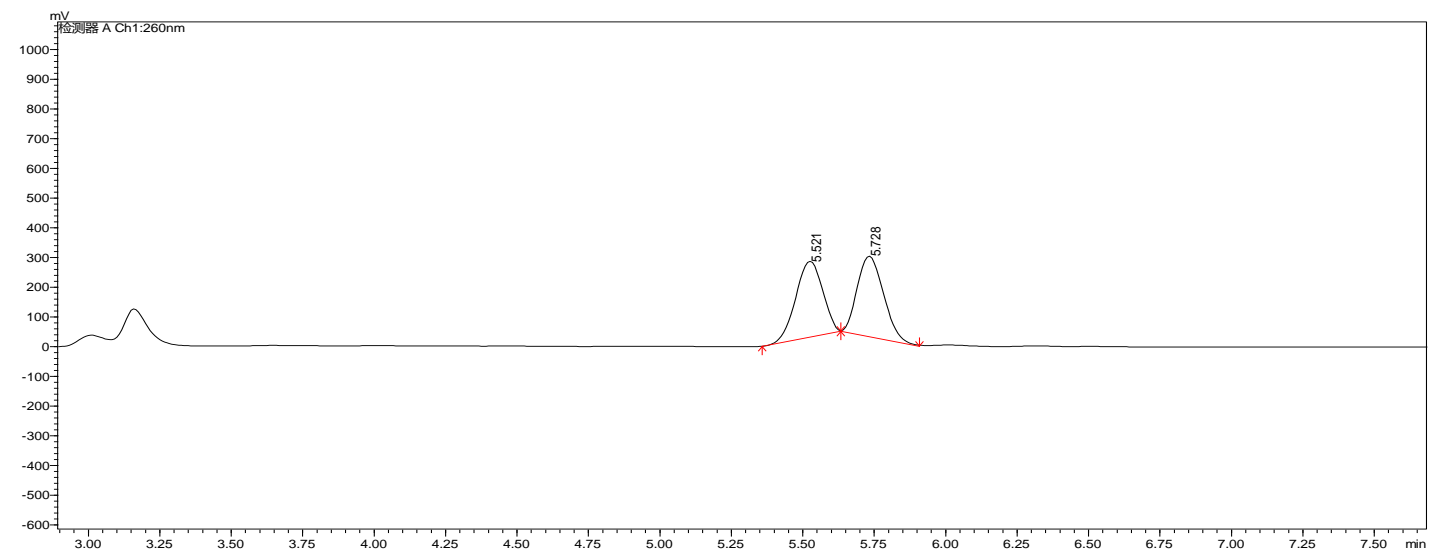

\begin{tabular}{|l|l|l|l|}
\hline Time & Conc. & Area & Height \\
\hline 5.521 & 49.98116 & 1682464 & 255907 \\
\hline 5.728 & 50.38751 & 1731127 & 269795 \\
\hline
\end{tabular}

Enantio-enriched sample:

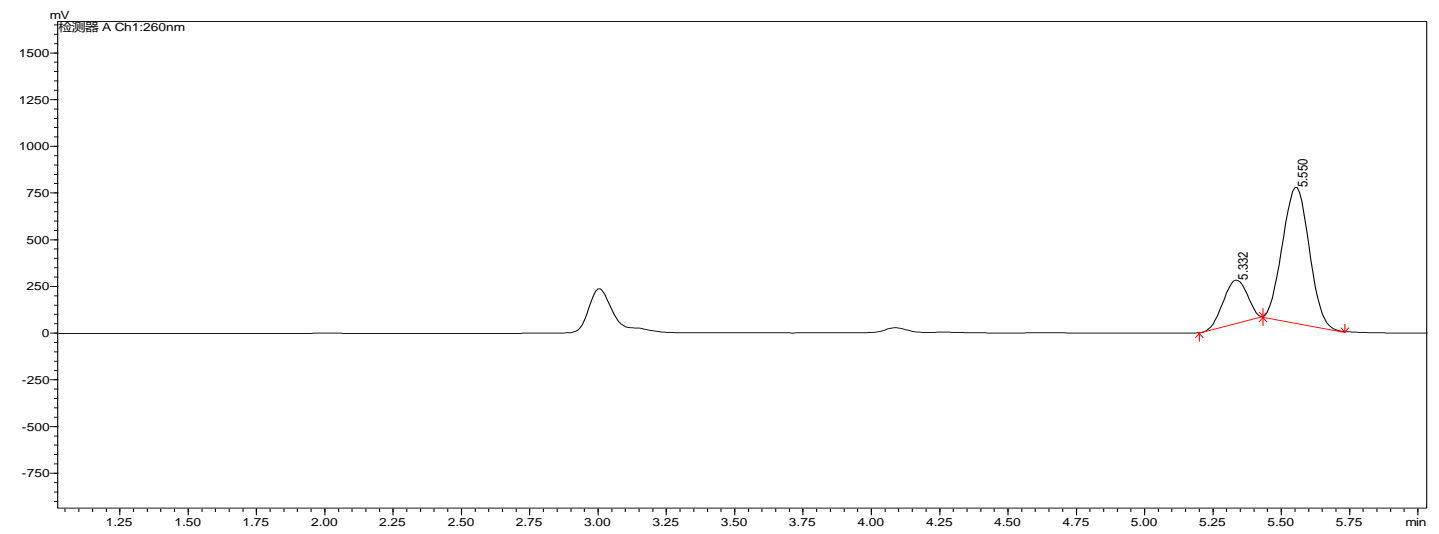

\begin{tabular}{|l|l|l|l|}
\hline Time & Conc. & Area & Height \\
\hline 5.332 & 22.54734 & 1437779 & 233883 \\
\hline 5.550 & 77.45266 & 4938934 & 725554 \\
\hline
\end{tabular}

Column: AD-H;

Eluent: $n$-Hexane: $i$-PrOH $=98: 2$;

Flow rate: $1 \mathrm{~mL} / \mathrm{min}$;

Pressure: 3.7 Mpa;

Wavelength: $260 \mathrm{~nm}$;

Temperature: $35^{\circ} \mathrm{C}$ 


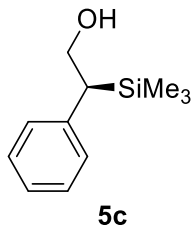

Racemic sample:

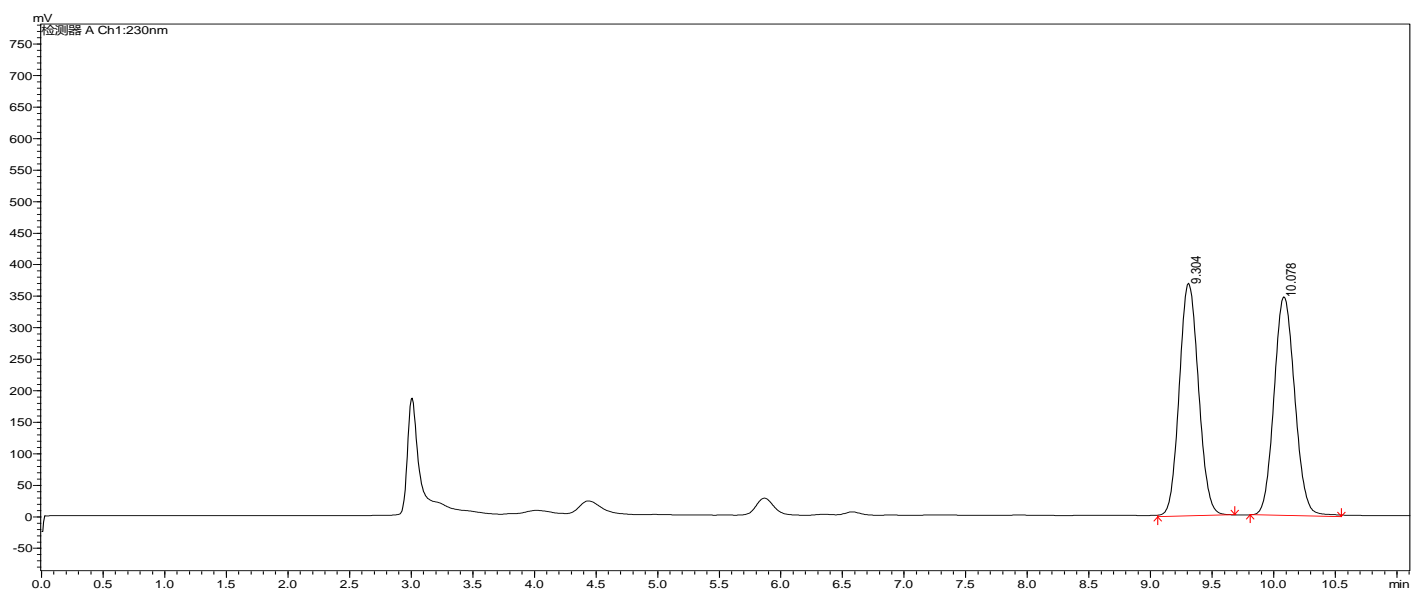

\begin{tabular}{|l|l|l|l|}
\hline Time & Conc. & Area & Height \\
\hline 9.304 & 49.66903 & 4019409 & 369258 \\
\hline 10.078 & 50.33097 & 4072975 & 346861 \\
\hline
\end{tabular}

Enantio-enriched sample:

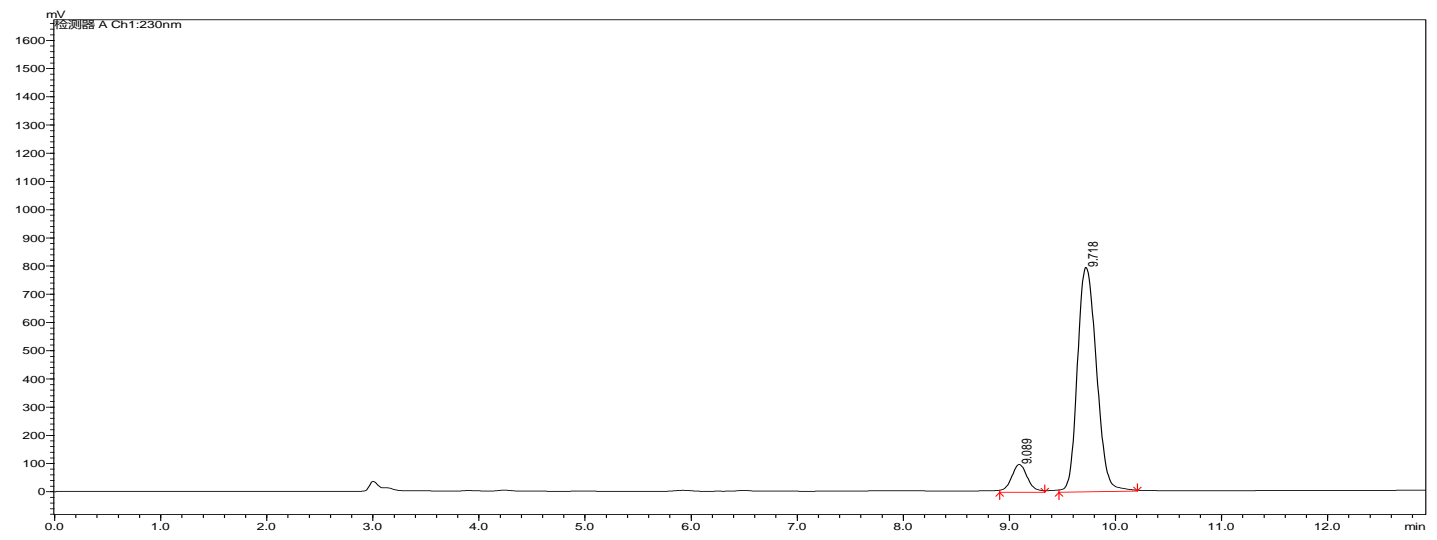

\begin{tabular}{|l|l|l|l|}
\hline Time & Conc. & Area & Height \\
\hline 9.089 & 9.42842 & 1036888 & 98064 \\
\hline 9.718 & 90.57158 & 9960588 & 795769 \\
\hline
\end{tabular}

Column: AD-H;

Eluent: $n$-Hexane: $i$-PrOH $=98: 2$;

Flow rate: $1 \mathrm{~mL} / \mathrm{min}$;

Pressure: 3.7 Mpa;

Wavelength: $240 \mathrm{~nm}$;

Temperature: $35^{\circ} \mathrm{C}$ 


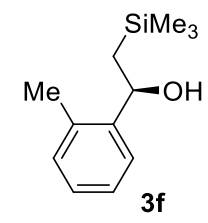

Racemic sample:

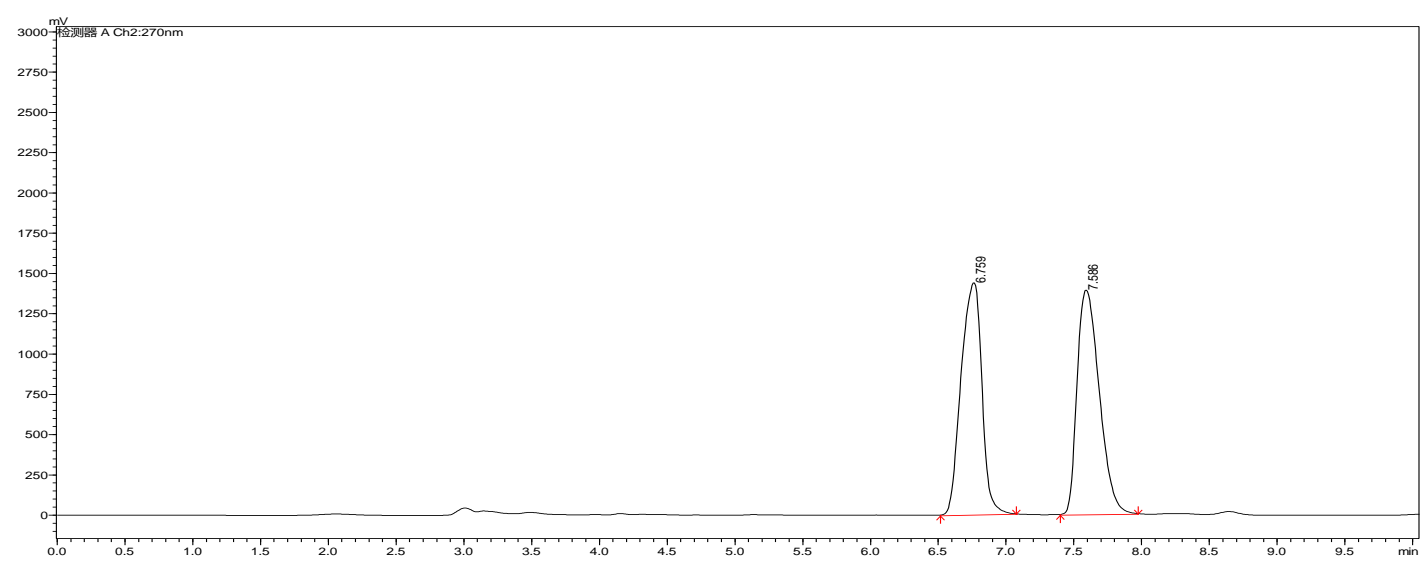

\begin{tabular}{|l|l|l|l|}
\hline Time & Conc. & Area & Height \\
\hline 6.757 & 49.03942 & 1114610 & 107865 \\
\hline 7.583 & 50.66058 & 1158276 & 103591 \\
\hline
\end{tabular}

Enantio-enriched sample:

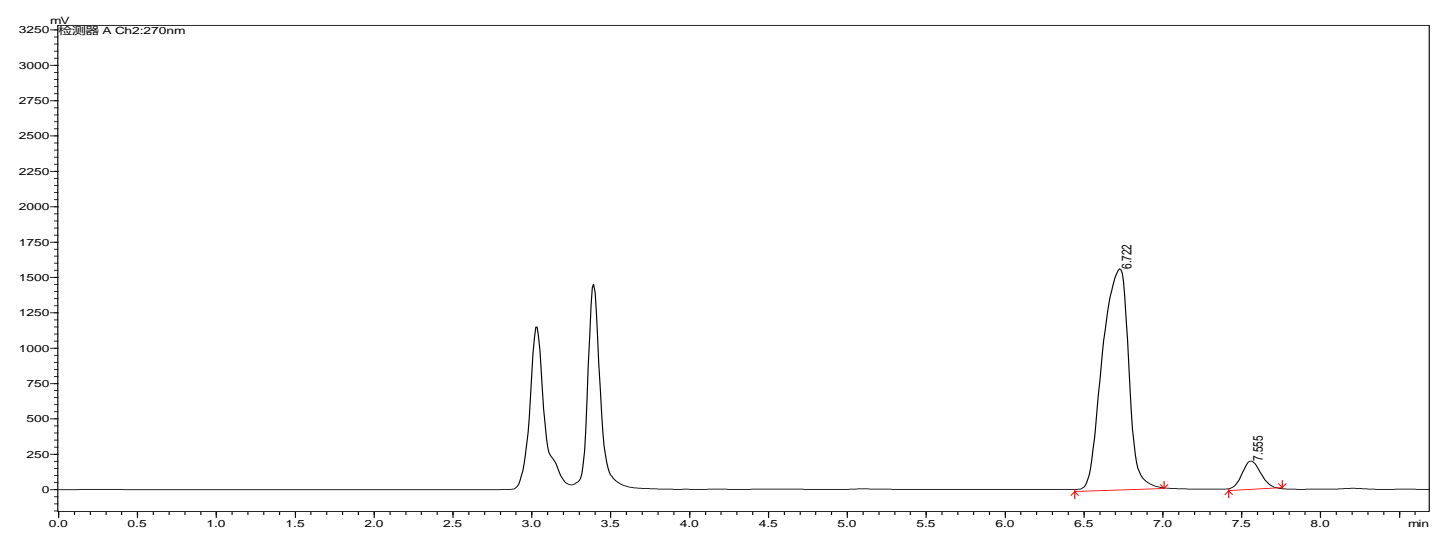

\begin{tabular}{|l|l|l|l|}
\hline Time & Conc. & Area & Height \\
\hline 6.722 & 91.60969 & 18137332 & 1563596 \\
\hline 7.555 & 8.39031 & 1661155 & 200435 \\
\hline
\end{tabular}

$83 \%$ ee

Column: AD-H;

Eluent: $n$-Hexane: $i$-PrOH $=98: 2$;

Flow rate: $1 \mathrm{~mL} / \mathrm{min}$;

Pressure: $4.1 \mathrm{Mpa}$;

Wavelength: $270 \mathrm{~nm}$;

Temperature: $35^{\circ} \mathrm{C}$ 
<smiles>Cc1cccc(C(O)COC(C)(C)C)c1</smiles>

Racemic sample:

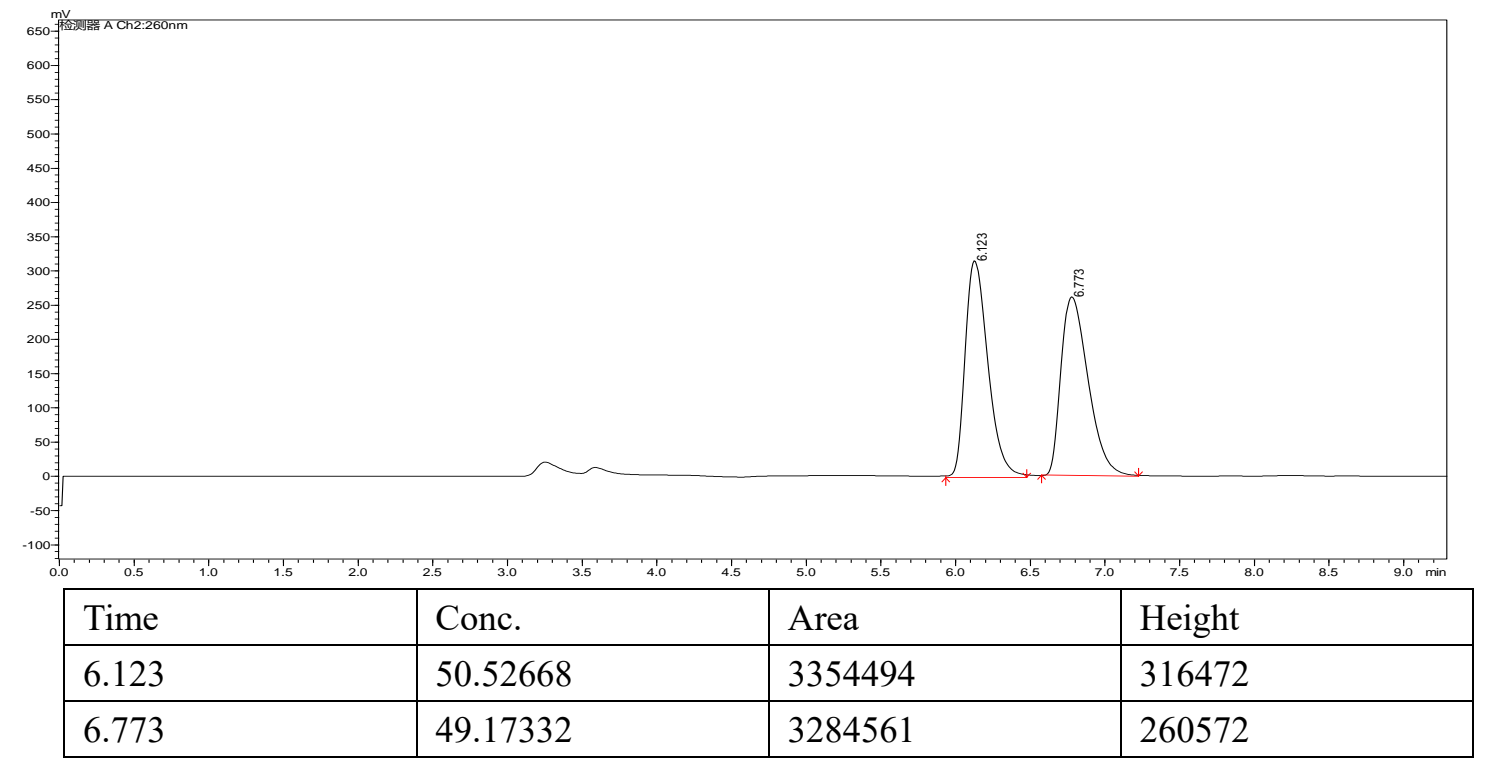

Enantio-enriched sample:

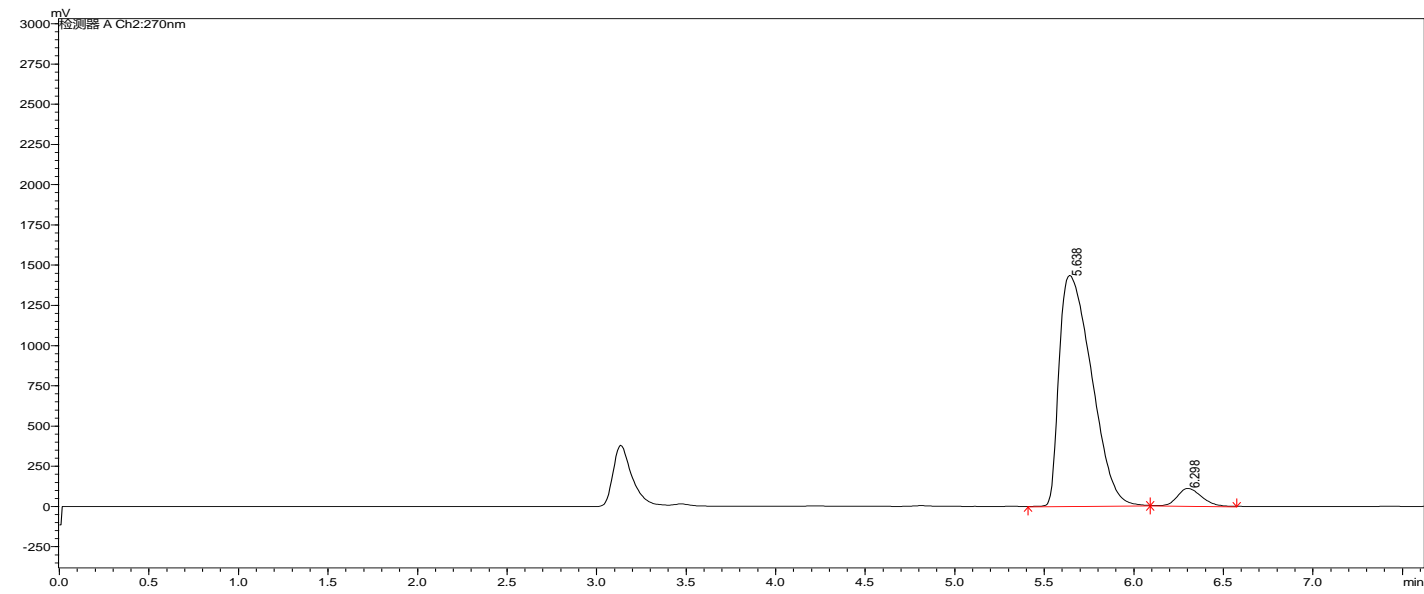

\begin{tabular}{|l|l|l|l|}
\hline Time & Conc. & Area & Height \\
\hline 5.638 & 94.33044 & 20117623 & 1436602 \\
\hline 6.298 & 4.36956 & 1088927 & 111546 \\
\hline
\end{tabular}

Column: AS-H;

Eluent: $n$-Hexane: $i$-PrOH $=98: 2$;

Flow rate: $1 \mathrm{~mL} / \mathrm{min}$;

Pressure: $4.2 \mathrm{Mpa}$;

Wavelength: $270 \mathrm{~nm}$;

Temperature: $35^{\circ} \mathrm{C}$ 


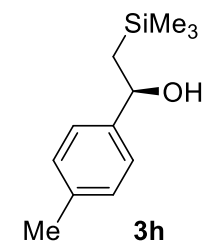

Racemic sample:

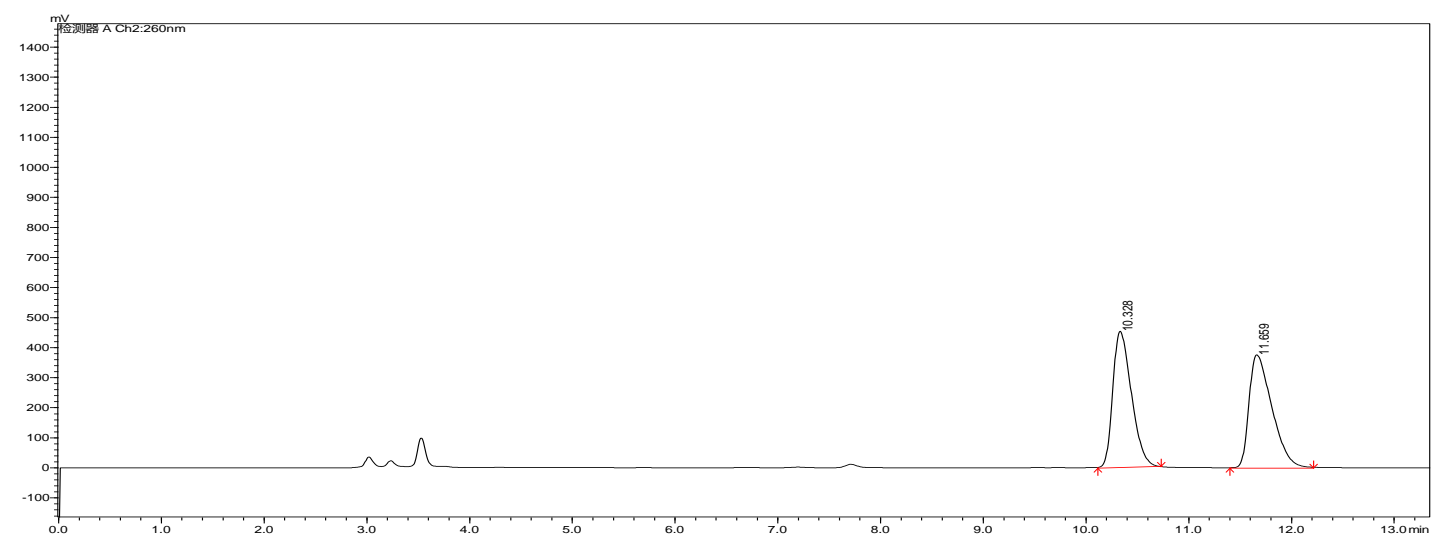

\begin{tabular}{|l|l|l|l|}
\hline Time & Conc. & Area & Height \\
\hline 10.328 & 49.84127 & 5726270 & 453496 \\
\hline 11.659 & 50.15873 & 5879167 & 376922 \\
\hline
\end{tabular}

Enantio-enriched sample:

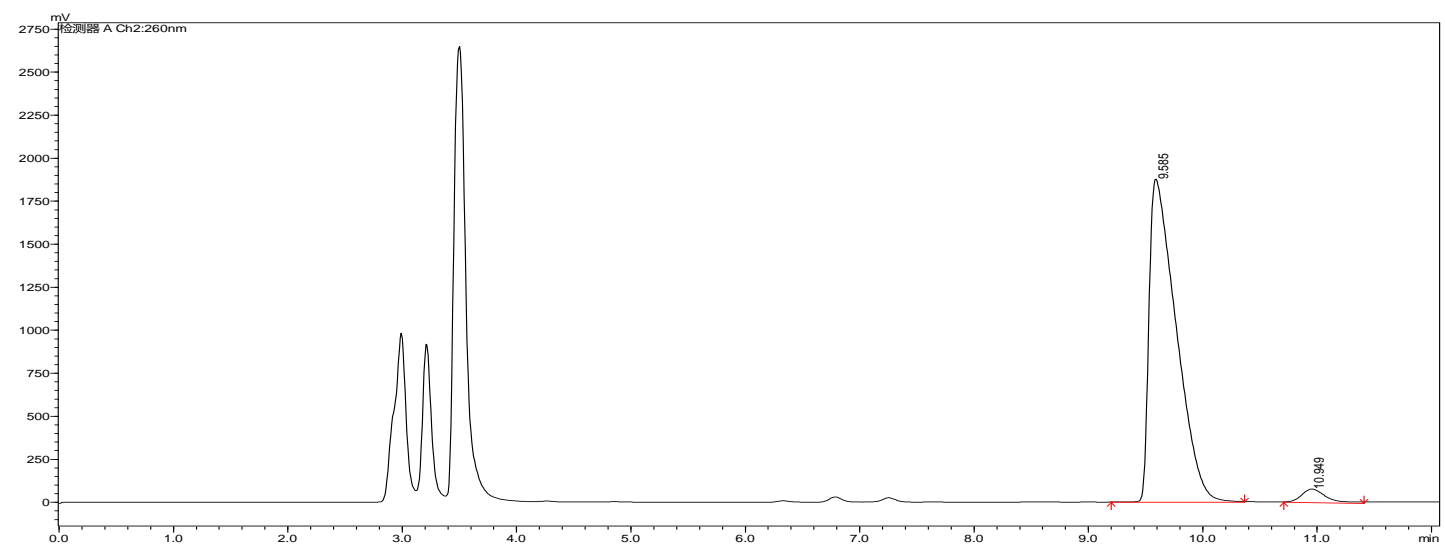

\begin{tabular}{|l|l|l|l|}
\hline Time & Conc. & Area & Height \\
\hline 9.585 & 96.24815 & 30893795 & 1878476 \\
\hline 10.949 & 3.75185 & 1204272 & 78933 \\
\hline
\end{tabular}

$93 \%$ ee

Column: AD-H;

Eluent: $n$-Hexane: $i$-PrOH $=98: 2$;

Flow rate: $1 \mathrm{~mL} / \mathrm{min}$;

Pressure: 4.1 Mpa;

Wavelength: $270 \mathrm{~nm}$;

Temperature: $35^{\circ} \mathrm{C}$ 


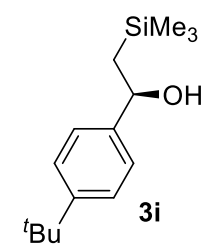

Racemic sample:

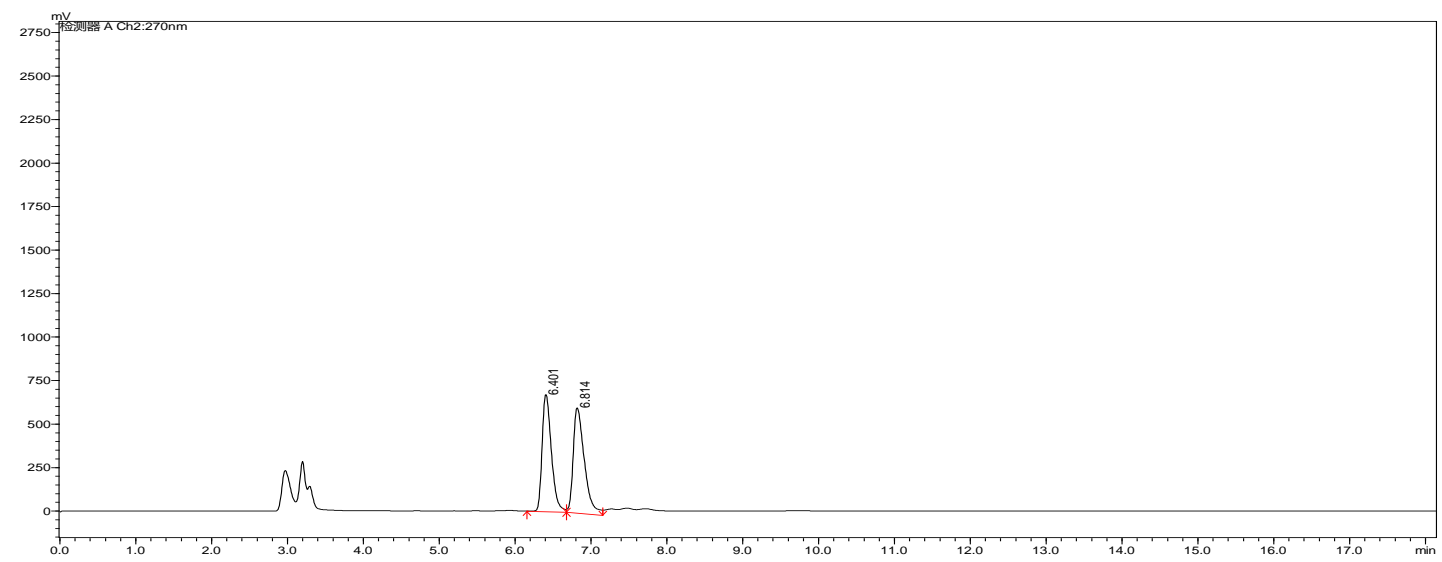

\begin{tabular}{|l|l|l|l|}
\hline Time & Conc. & Area & Height \\
\hline 6.401 & 49.06119 & 5763601 & 673055 \\
\hline 6.814 & 50.93881 & 6228614 & 606175 \\
\hline
\end{tabular}

Enantio-enriched sample:

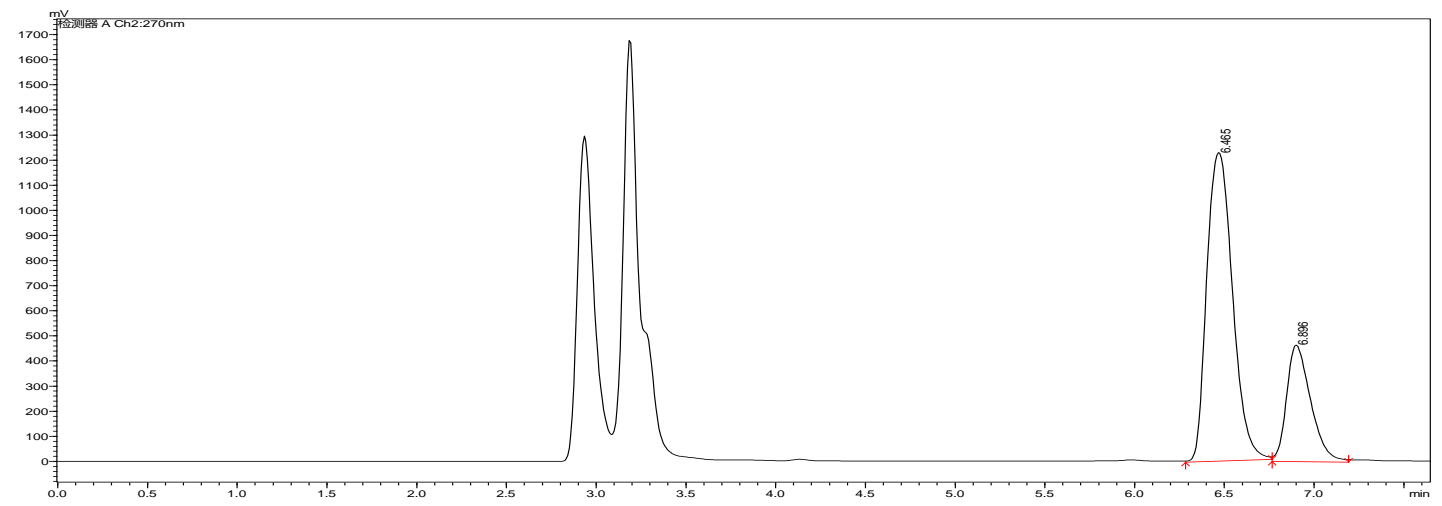

\begin{tabular}{|l|l|l|l|}
\hline Time & Conc. & Area & Height \\
\hline 6.465 & 73.87126 & 12254133 & 1229443 \\
\hline 6.896 & 26.12874 & 4334366 & 463745 \\
\hline
\end{tabular}

$48 \%$ ee

Column: AD-H;

Eluent: $n$-Hexane: $i$-PrOH $=98: 2$;

Flow rate: $1 \mathrm{~mL} / \mathrm{min}$;

Pressure: $4.0 \mathrm{Mpa}$;

Wavelength: $270 \mathrm{~nm}$;

Temperature: $35^{\circ} \mathrm{C}$ 


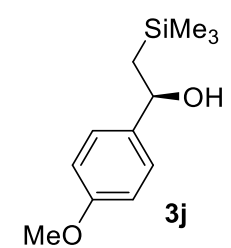

Racemic sample:

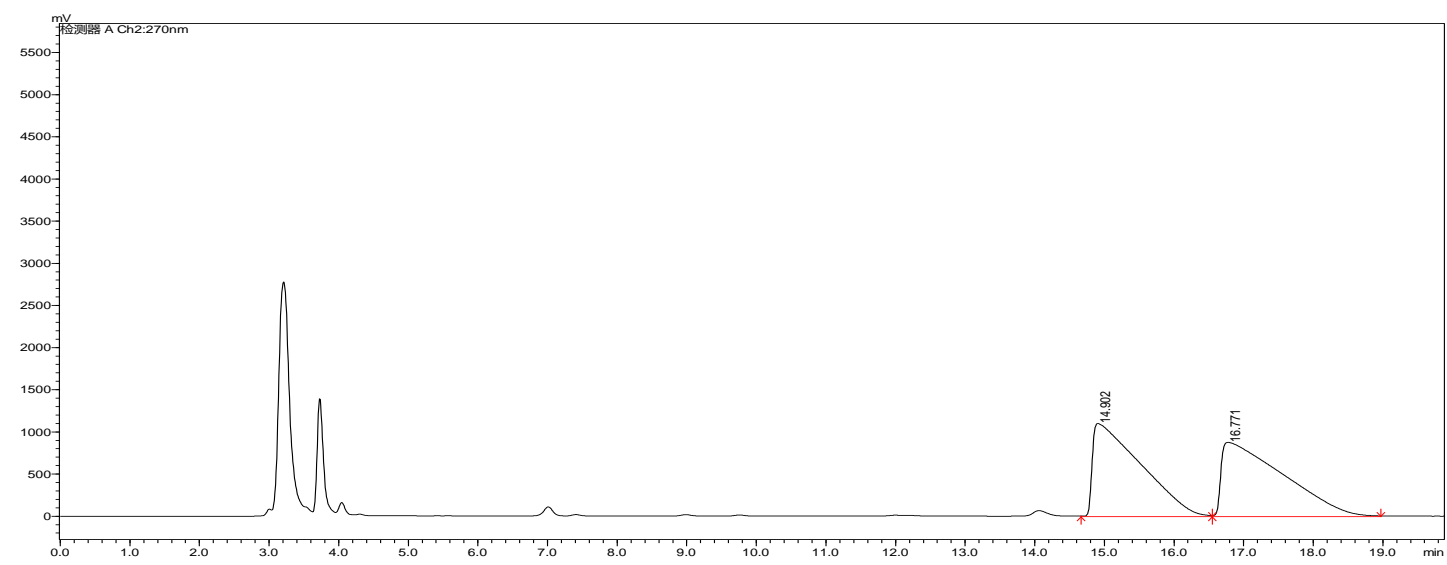

\begin{tabular}{|l|l|l|l|}
\hline Time & Conc. & Area & Height \\
\hline 14.902 & 49.43464 & 53423259 & 1105605 \\
\hline 16.771 & 50.06536 & 54645215 & 881200 \\
\hline
\end{tabular}

Enantio-enriched sample:

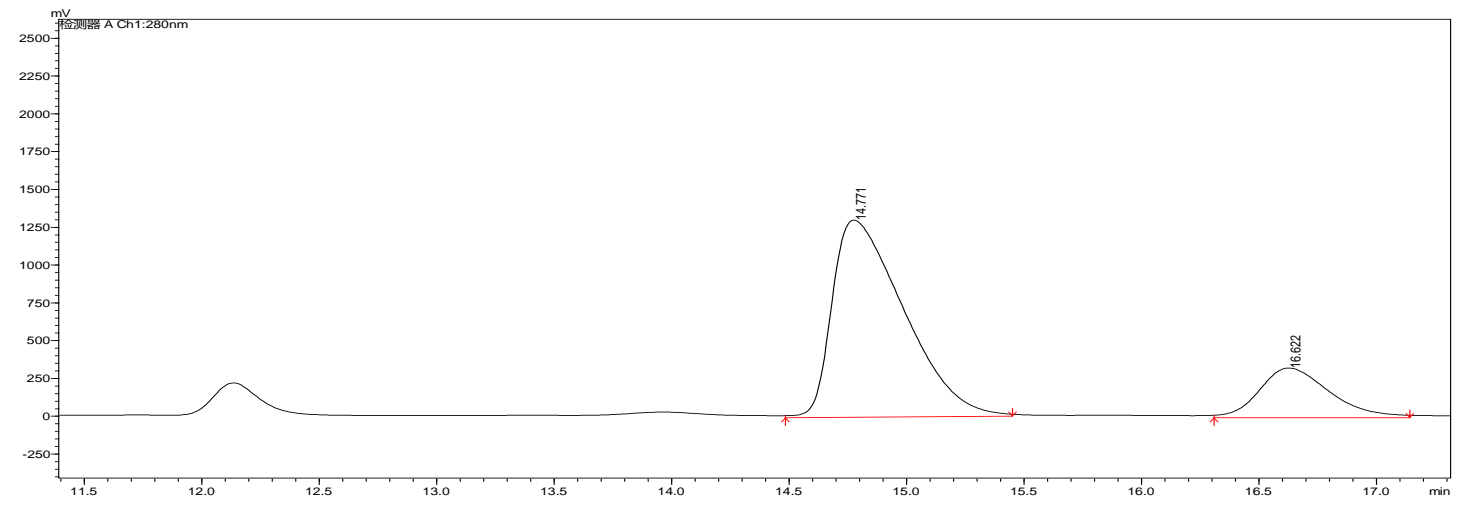

\begin{tabular}{|l|l|l|l|}
\hline Time & Conc. & Area & Height \\
\hline 14.771 & 82.47320 & 27673849 & 1304161 \\
\hline 16.622 & 17.52680 & 6908313 & 329039 \\
\hline
\end{tabular}

Column: AD-H;

Eluent: $n$-Hexane: $i$-PrOH $=98: 2$;

Flow rate: $1 \mathrm{~mL} / \mathrm{min}$;

Pressure: $4.2 \mathrm{Mpa}$;

Wavelength: $270 \mathrm{~nm}$;

Temperature: $35^{\circ} \mathrm{C}$ 


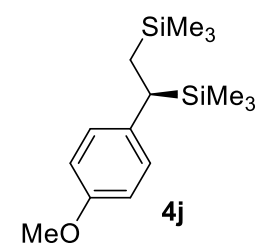

Racemic sample:

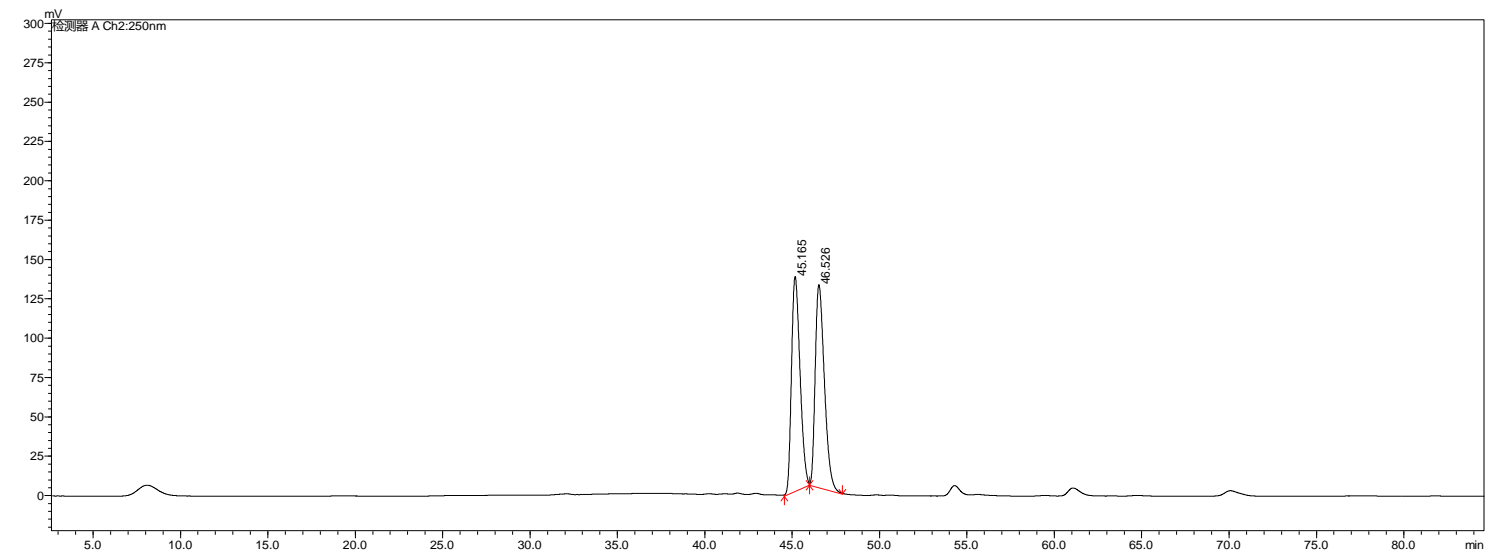

\begin{tabular}{|l|l|l|l|}
\hline Time & Conc. & Area & Height \\
\hline 45.163 & 49.94704 & 28420545 & 863671 \\
\hline 46.525 & 50.05296 & 28480815 & 813750 \\
\hline
\end{tabular}

Enantio-enriched sample:

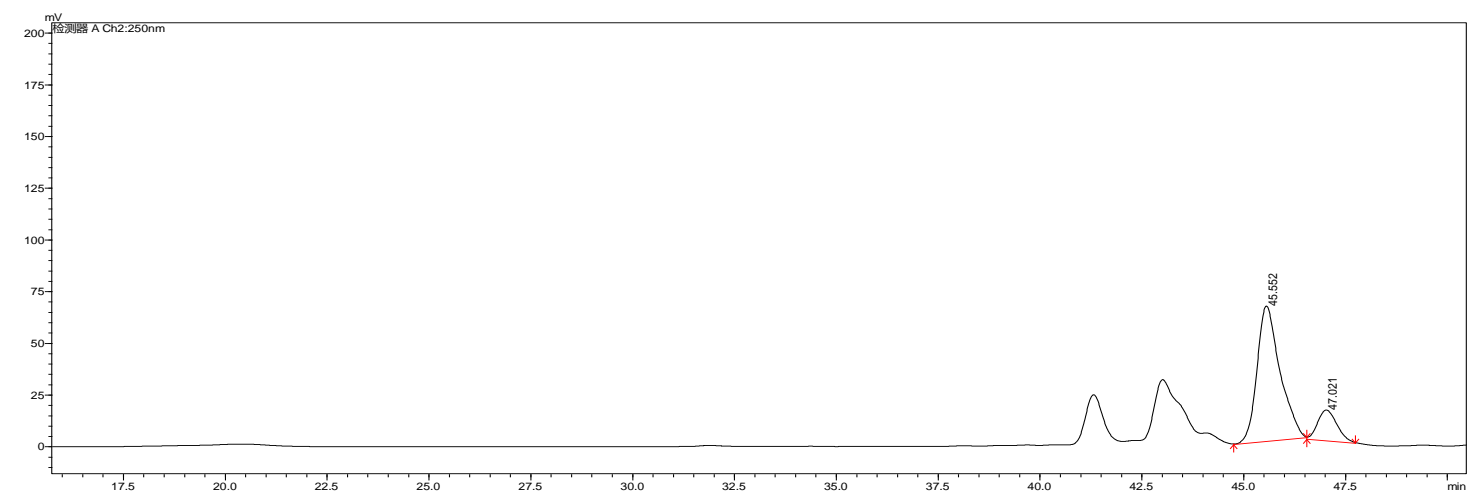

\begin{tabular}{|l|l|l|l|}
\hline Time & Conc. & Area & Height \\
\hline 45.552 & 84.02534 & 2576865 & 65474 \\
\hline 47.021 & 15.97466 & 489906 & 14825 \\
\hline
\end{tabular}

Column: OD-H*3;

Eluent: $n$-Hexane: $i-\mathrm{PrOH}=0.3: 0.0$;

Flow rate: $0.3 \mathrm{~mL} / \mathrm{min}$;

Pressure: $3.1 \mathrm{Mpa}$;

Wavelength: $250 \mathrm{~nm}$;

Temperature: $16^{\circ} \mathrm{C}$ 


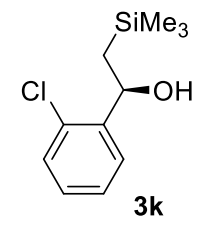

Racemic sample:

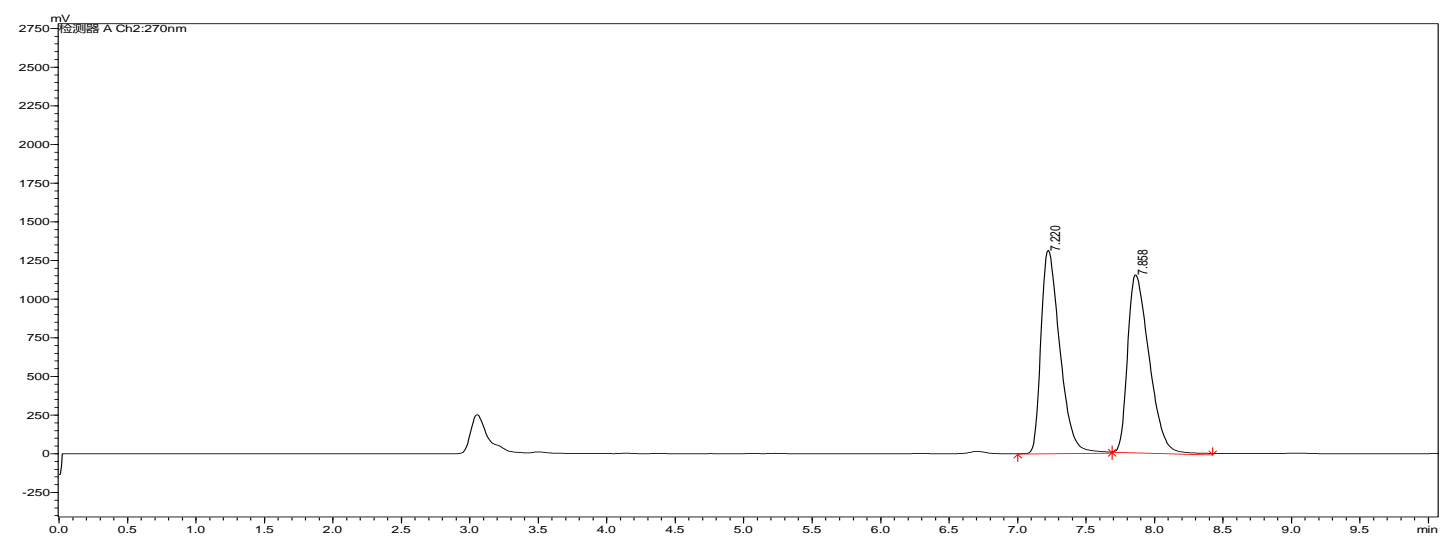

\begin{tabular}{|l|l|l|l|}
\hline Time & Conc. & Area & Height \\
\hline 7.220 & 49.94979 & 12670608 & 1316478 \\
\hline 7.858 & 50.05021 & 12696082 & 1150478 \\
\hline
\end{tabular}

Enantio-enriched sample:

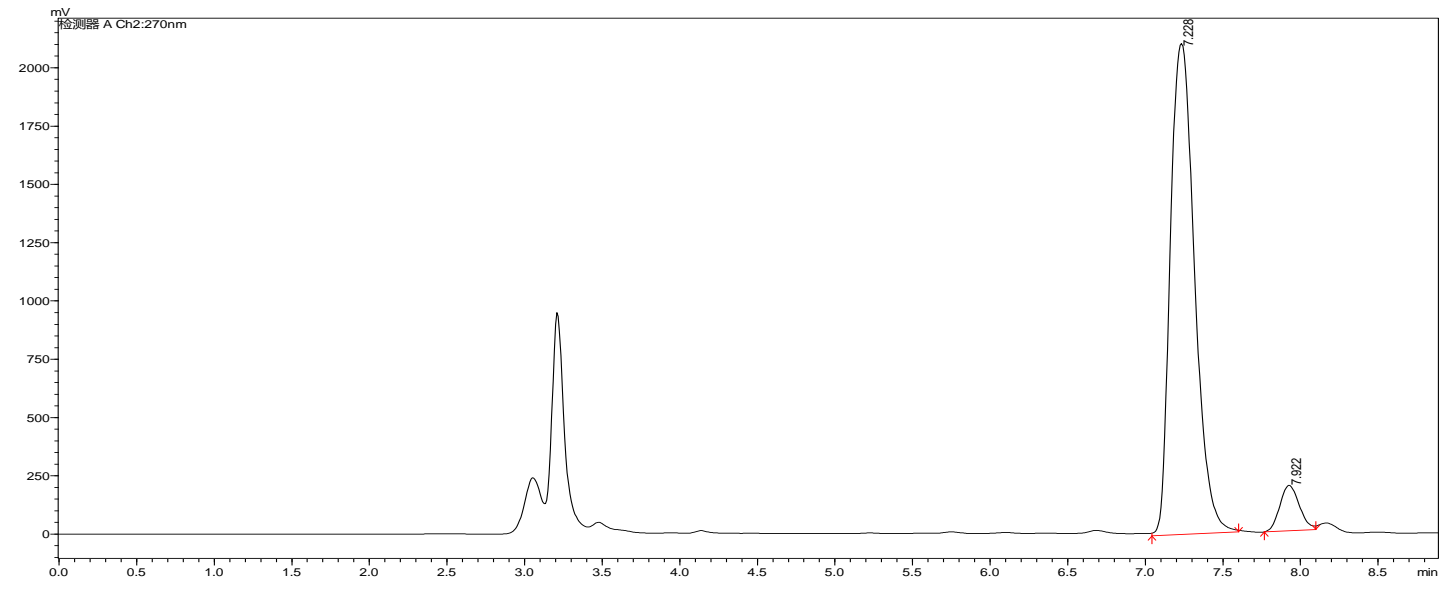

\begin{tabular}{|l|l|l|l|}
\hline Time & Conc. & Area & Height \\
\hline 7.228 & 93.03323 & 22904413 & 2106034 \\
\hline 7.922 & 6.96677 & 1715192 & 194783 \\
\hline
\end{tabular}

$86 \%$ ee

Column: AD-H;

Eluent: $n$-Hexane: $i$-PrOH $=98: 2$;

Flow rate: $1 \mathrm{~mL} / \mathrm{min}$;

Pressure: $4.0 \mathrm{Mpa}$;

Wavelength: $270 \mathrm{~nm}$;

Temperature: $35^{\circ} \mathrm{C}$ 


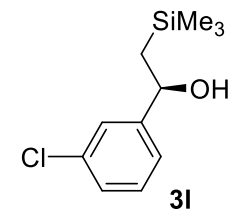

Racemic sample:

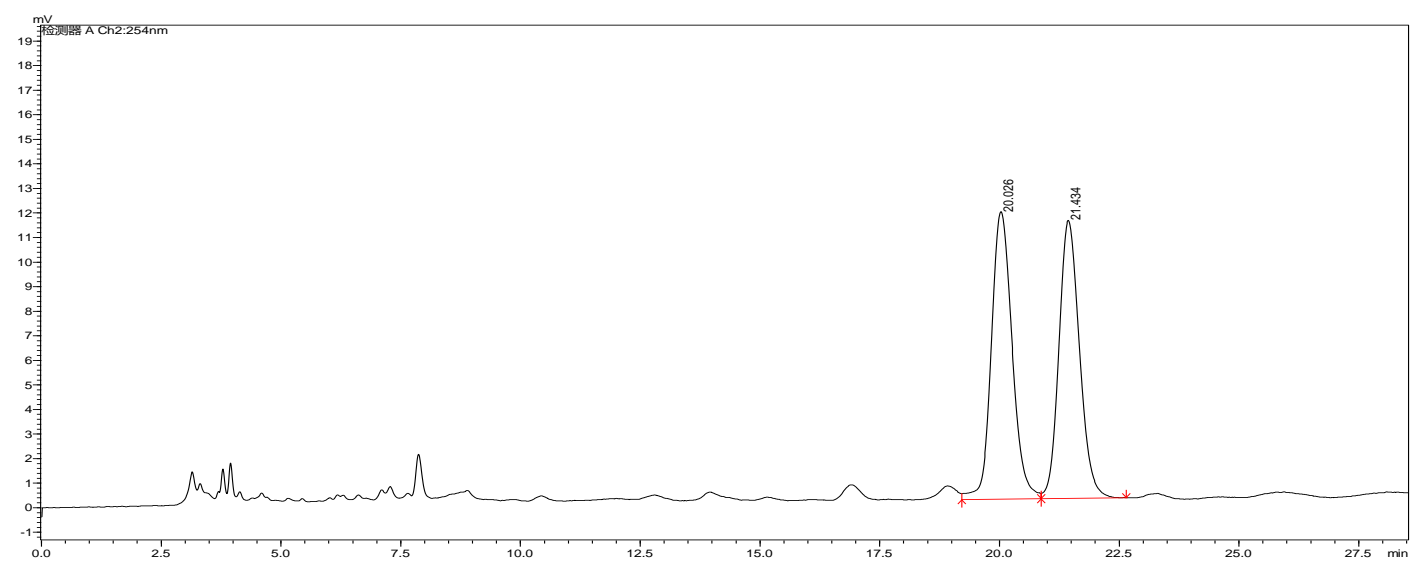

\begin{tabular}{|l|l|l|l|}
\hline Time & Conc. & Area & Height \\
\hline 20.024 & 50.04332 & 501653 & 16571 \\
\hline 21.431 & 49.95673 & 481145 & 16115 \\
\hline
\end{tabular}

Enantio-enriched sample:

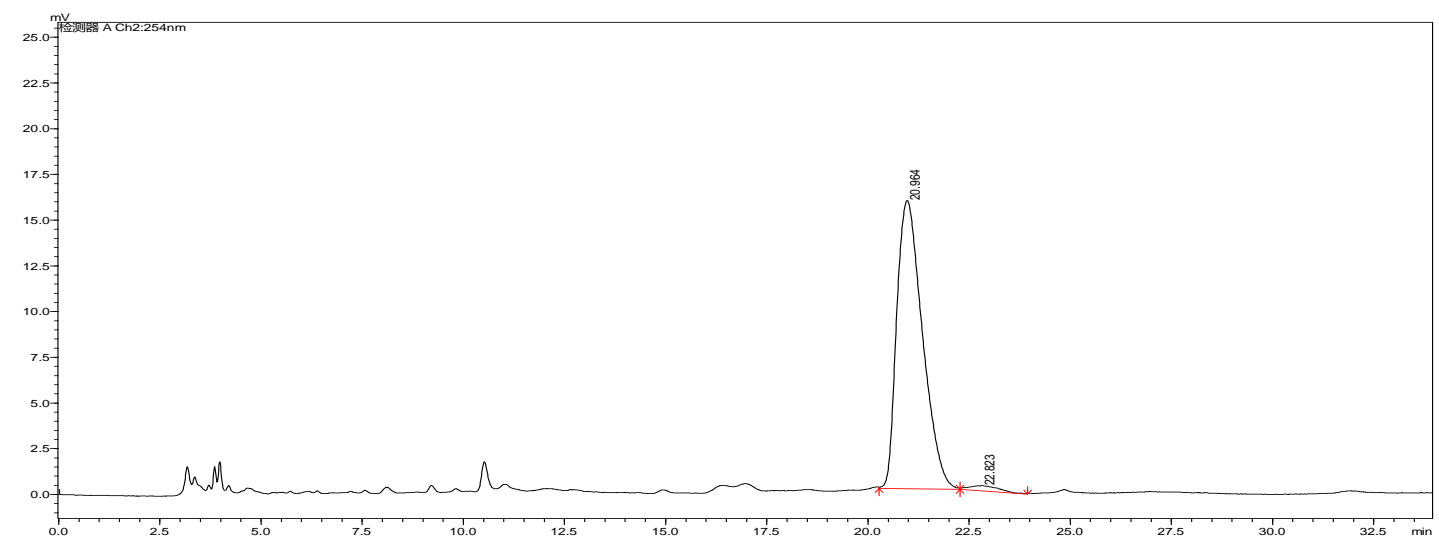

\begin{tabular}{|l|l|l|l|}
\hline Time & Conc. & Area & Height \\
\hline 20.964 & 97.84885 & 696691 & 15767 \\
\hline 22.823 & 2.15127 & 15317 & 299 \\
\hline
\end{tabular}

$96 \%$ ee

Column: AD-H;

Eluent: $n$-Hexane: $i$-PrOH $=99.5: 0.5$;

Flow rate: $1 \mathrm{~mL} / \mathrm{min}$;

Pressure: $3.8 \mathrm{Mpa}$;

Wavelength: $260 \mathrm{~nm}$;

Temperature: $35^{\circ} \mathrm{C}$ 


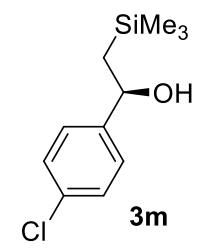

Racemic sample:

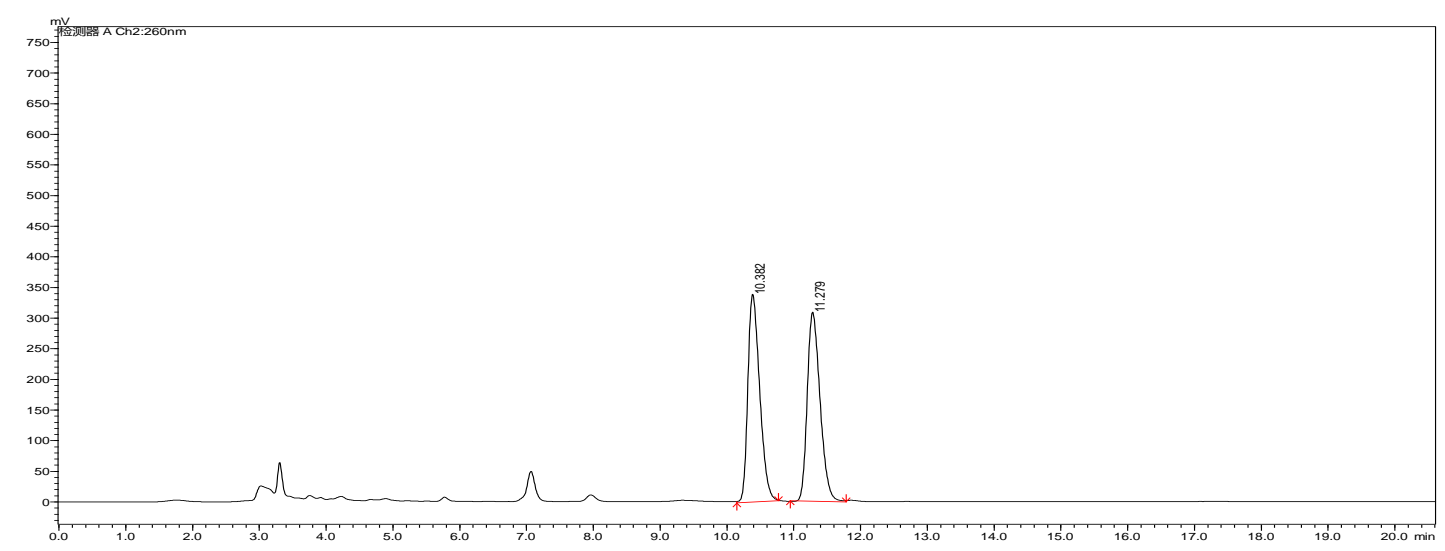

\begin{tabular}{|l|l|l|l|}
\hline Time & Conc. & Area & Height \\
\hline 10.382 & 50.45888 & 4187376 & 339095 \\
\hline 11.279 & 49.94112 & 4111216 & 308503 \\
\hline
\end{tabular}

Enantio-enriched sample:

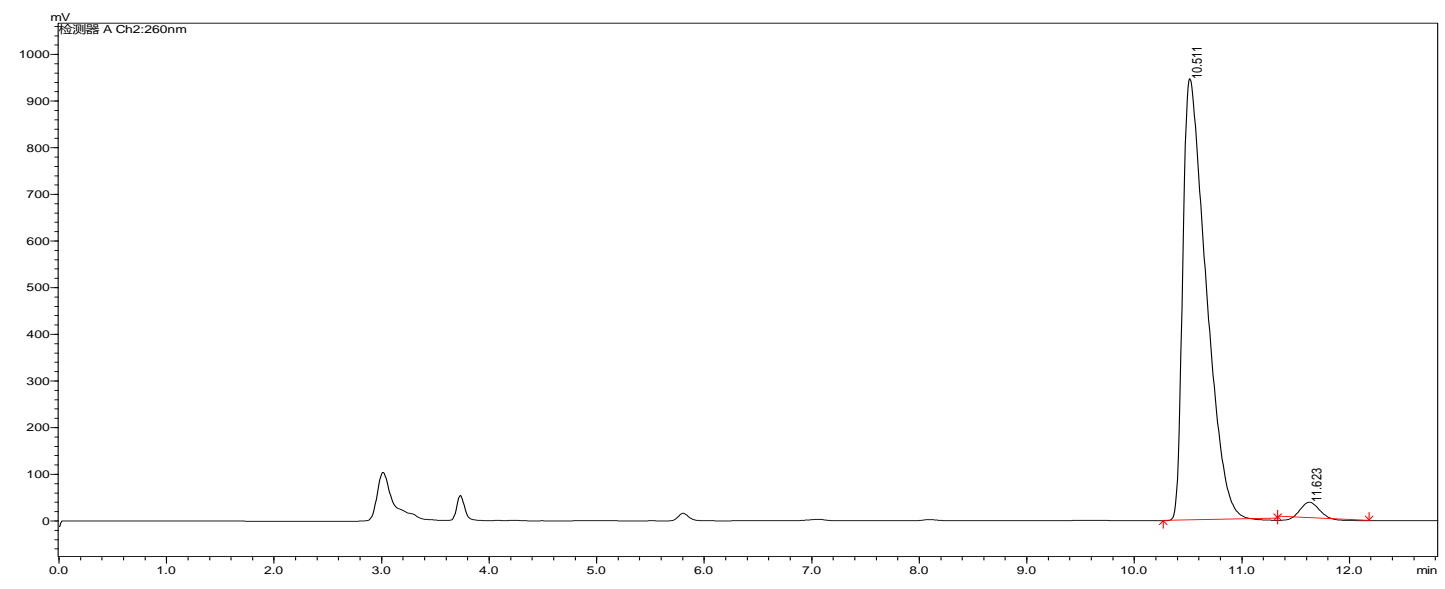

\begin{tabular}{|l|l|l|l|}
\hline Time & Conc. & Area & Height \\
\hline 10.511 & 98.09658 & 14148918 & 945515 \\
\hline 11.623 & 1.90342 & 274538 & 32869 \\
\hline
\end{tabular}

Column: AD-H;

Eluent: $n$-Hexane: $i$-PrOH $=98: 2$;

Flow rate: $1 \mathrm{~mL} / \mathrm{min}$;

Pressure: 3.7 Mpa;

Wavelength: $260 \mathrm{~nm}$;

Temperature: $35^{\circ} \mathrm{C}$ 


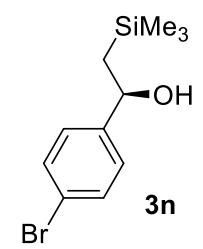

Racemic sample:

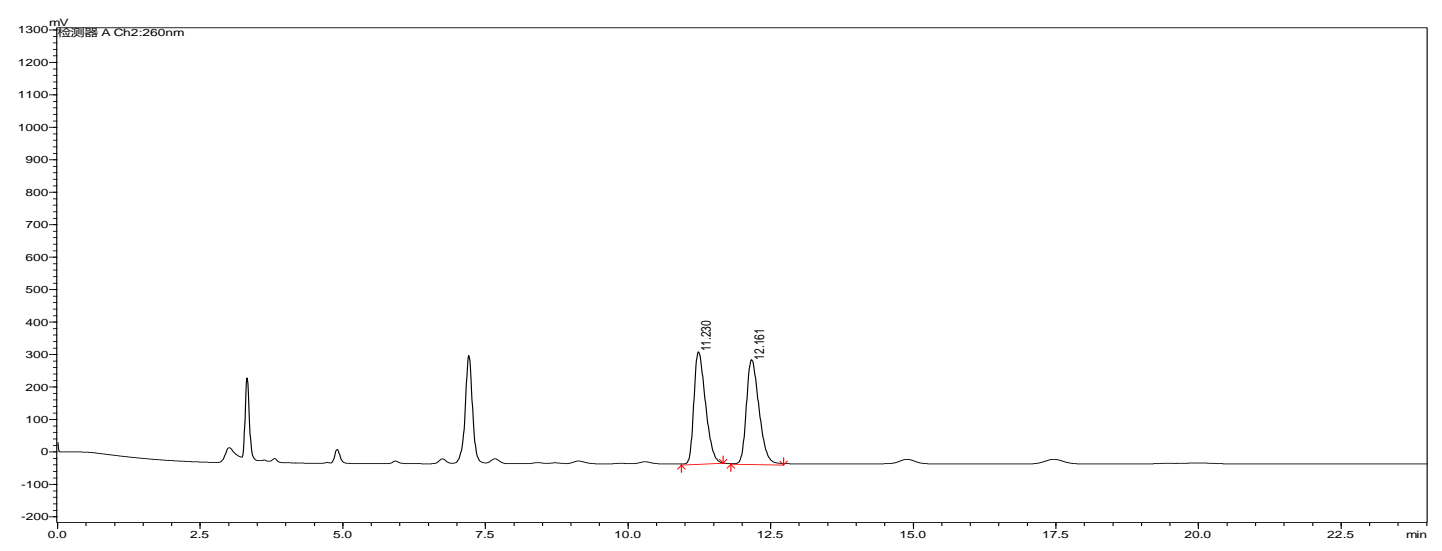

\begin{tabular}{|l|l|l|l|}
\hline Time & Conc. & Area & Height \\
\hline 11.228 & 50.07884 & 4707547 & 329760 \\
\hline 12.160 & 49.92116 & 4692725 & 305747 \\
\hline
\end{tabular}

Enantio-enriched sample:

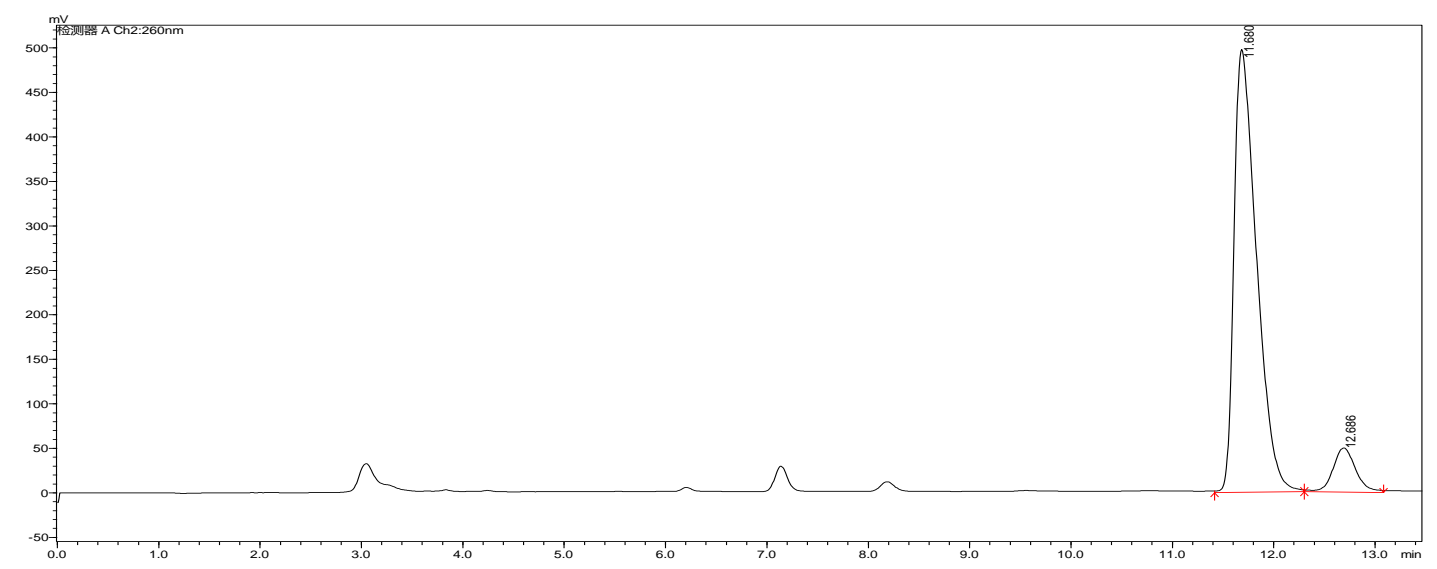

\begin{tabular}{|l|l|l|l|}
\hline Time & Conc. & Area & Height \\
\hline 11.680 & 90.85398 & 7682669 & 497598 \\
\hline 12.686 & 9.14602 & 773393 & 49479 \\
\hline
\end{tabular}

$82 \%$ ee

Column: AD-H;

Eluent: $n$-Hexane: $i$-PrOH $=98: 2$;

Flow rate: $1 \mathrm{~mL} / \mathrm{min}$;

Pressure: $3.7 \mathrm{Mpa}$;

Wavelength: $260 \mathrm{~nm}$;

Temperature: $35^{\circ} \mathrm{C}$ 


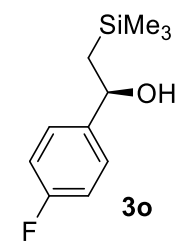

Racemic sample:

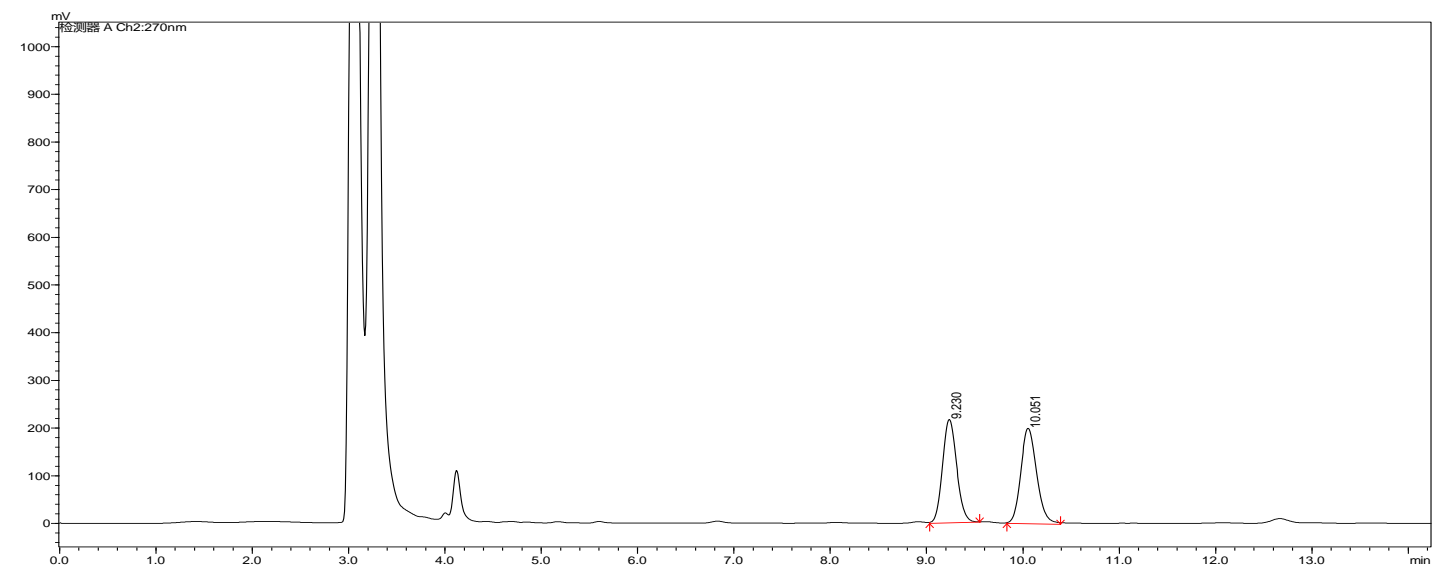

\begin{tabular}{|l|l|l|l|}
\hline Time & Conc. & Area & Height \\
\hline 9.230 & 49.19753 & 2227364 & 216993 \\
\hline 10.051 & 50.30247 & 2300026 & 199720 \\
\hline
\end{tabular}

Enantio-enriched sample:

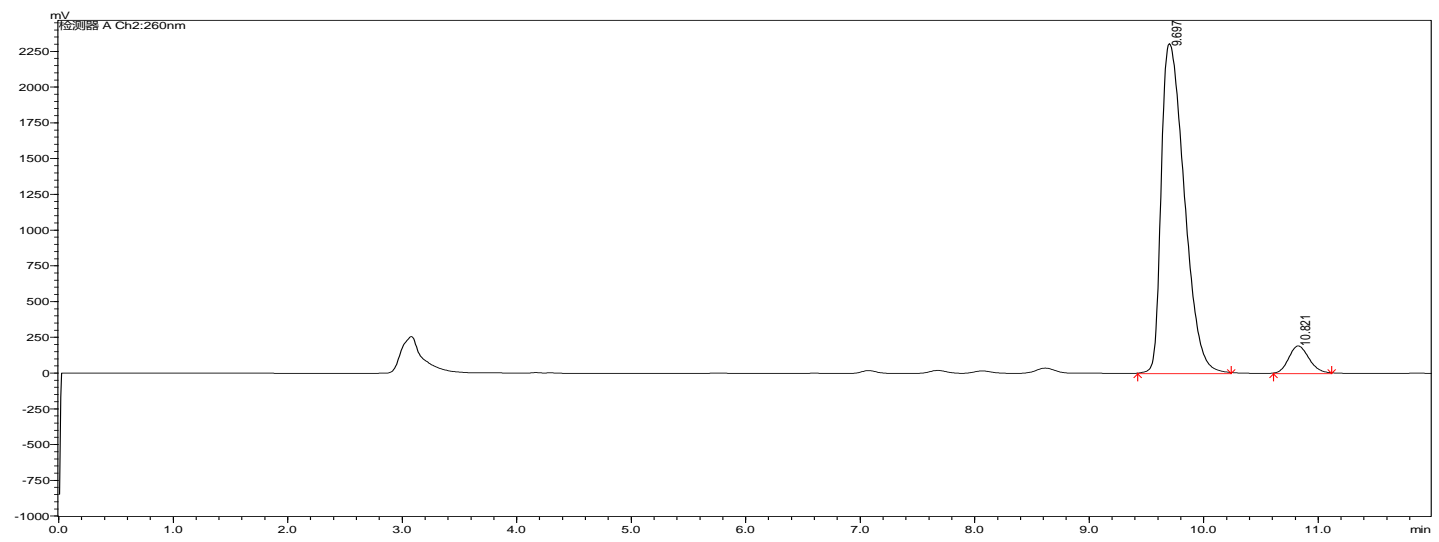

\begin{tabular}{|l|l|l|l|}
\hline Time & Conc. & Area & Height \\
\hline 9.697 & 92.97562 & 32386882 & 2309105 \\
\hline 10.821 & 7.02438 & 2446854 & 194645 \\
\hline
\end{tabular}

$86 \%$ ee

Column: AD-H;

Eluent: $n$-Hexane: $i$-PrOH $=98: 2$;

Flow rate: $1 \mathrm{~mL} / \mathrm{min}$;

Pressure: $3.8 \mathrm{Mpa}$;

Wavelength: $260 \mathrm{~nm}$;

Temperature: $35^{\circ} \mathrm{C}$ 


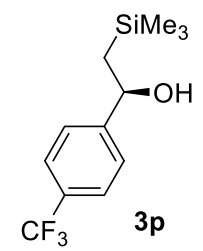

Racemic sample:

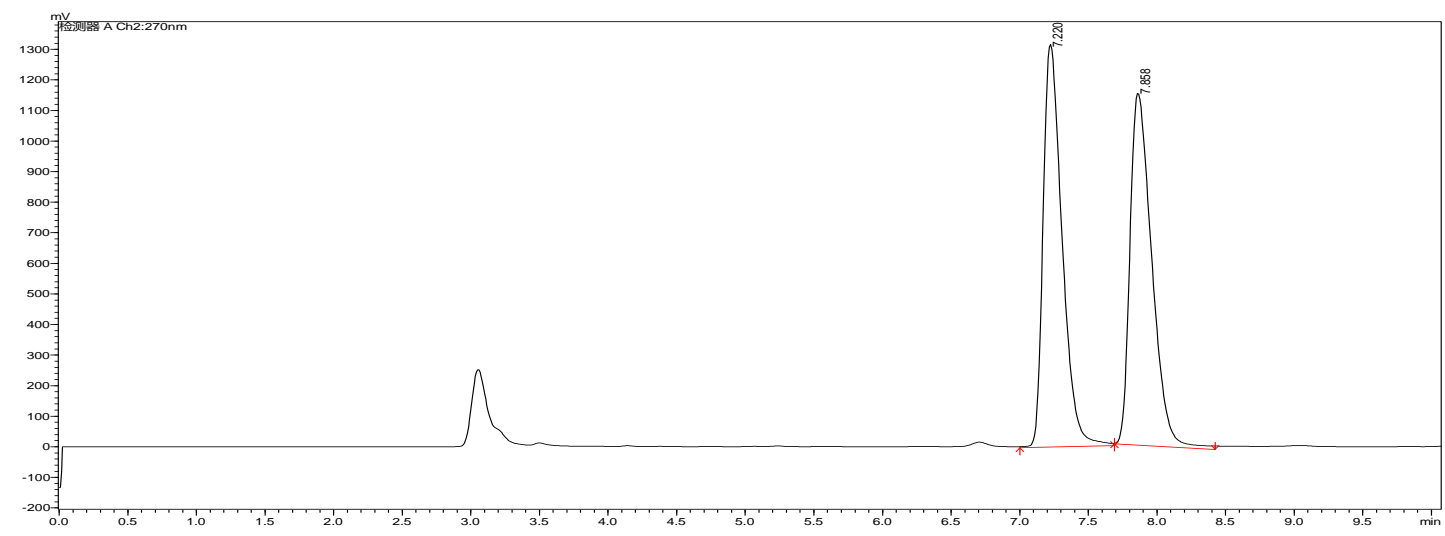

\begin{tabular}{|l|l|l|l|}
\hline Time & Conc. & Area & Height \\
\hline 7.220 & 49.94979 & 12670608 & 1316478 \\
\hline 7.858 & 50.05021 & 12696082 & 1150478 \\
\hline
\end{tabular}

Enantio-enriched sample:

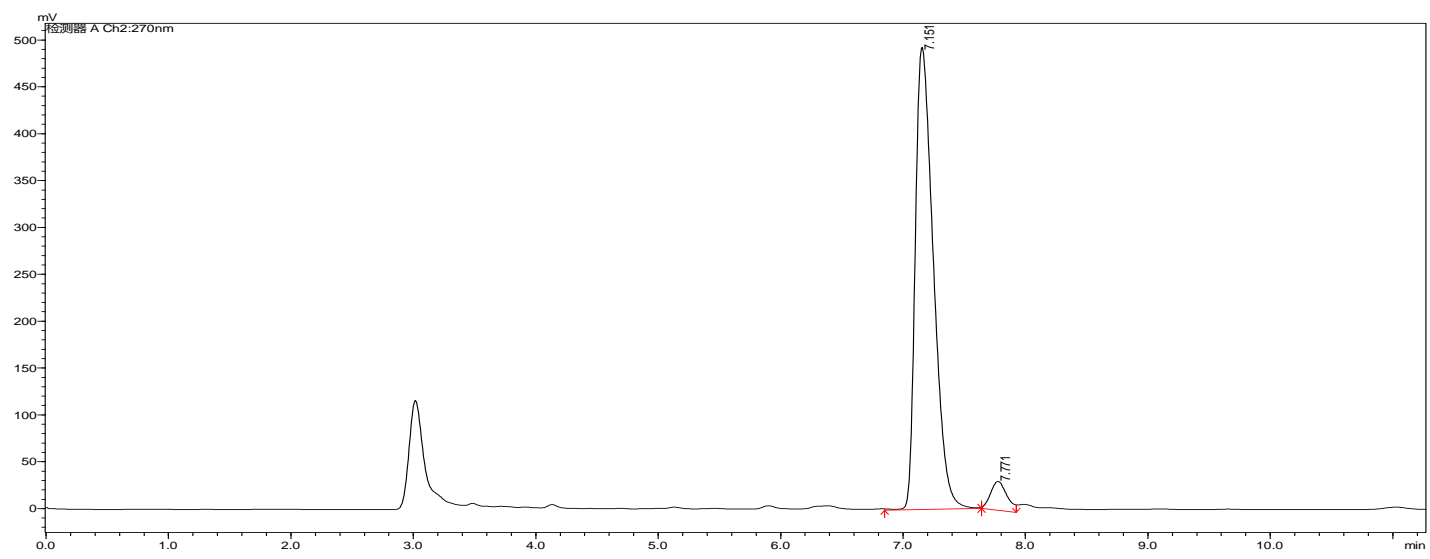

\begin{tabular}{|l|l|l|l|}
\hline Time & Conc. & Area & Height \\
\hline 7.151 & 94.99590 & 5448917 & 493365 \\
\hline 7.771 & 5.10410 & 288436 & 30701 \\
\hline
\end{tabular}

$90 \%$ ee

Column: AD-H;

Eluent: $n$-Hexane: $i$-PrOH $=98: 2$;

Flow rate: $1 \mathrm{~mL} / \mathrm{min}$;

Pressure: 4.1 Mpa;

Wavelength: $270 \mathrm{~nm}$;

Temperature: $35^{\circ} \mathrm{C}$ 


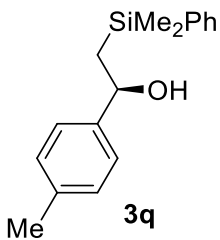

Racemic sample:

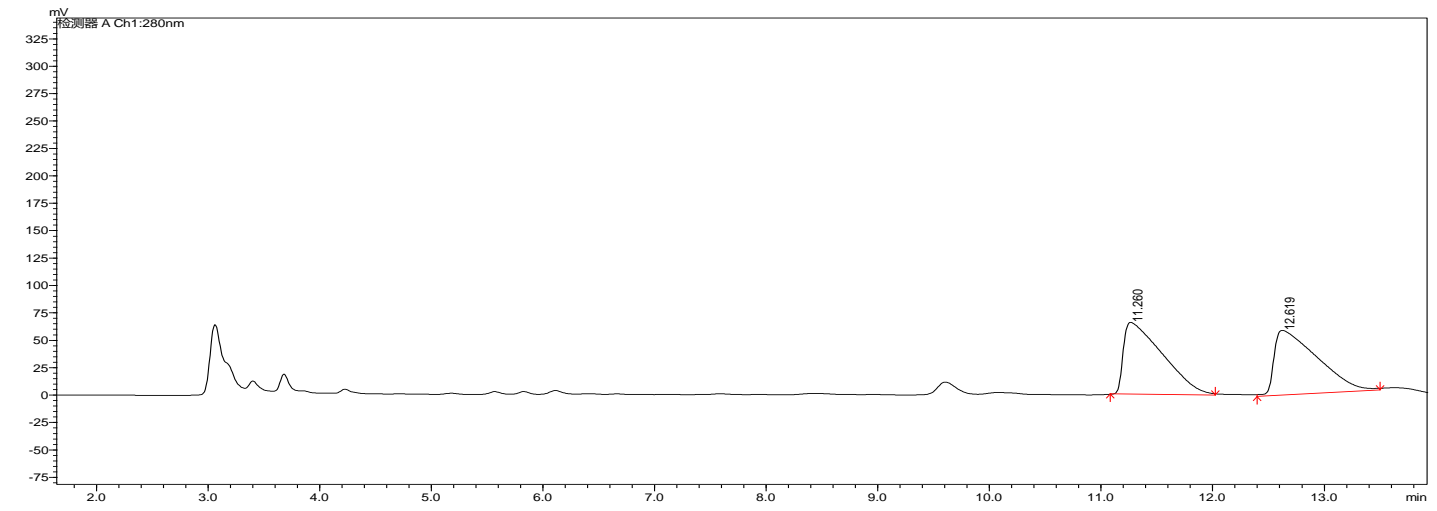

\begin{tabular}{|l|l|l|l|}
\hline Time & Conc. & Area & Height \\
\hline 11.260 & 50.06843 & 1584298 & 65373 \\
\hline 12.619 & 49.93157 & 1579968 & 58944 \\
\hline
\end{tabular}

Enantio-enriched sample:

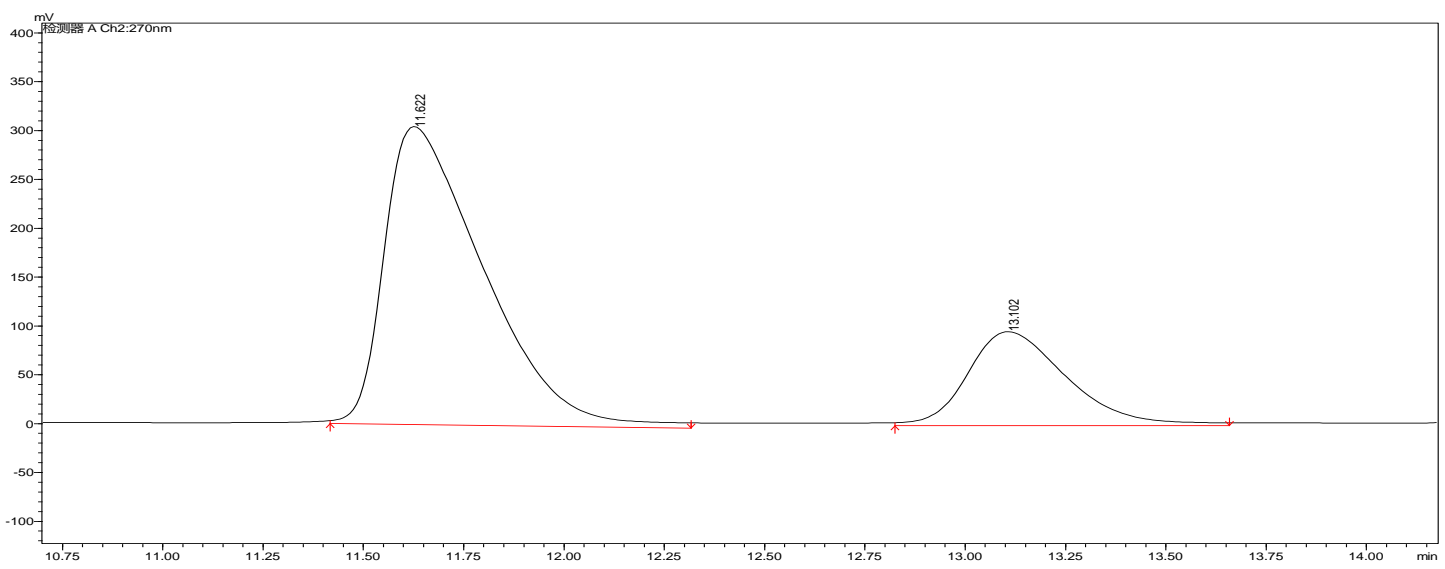

\begin{tabular}{|l|l|l|l|}
\hline Time & Conc. & Area & Height \\
\hline 11.622 & 79.88482 & 5239218 & 305181 \\
\hline 13.102 & 20.21518 & 1669882 & 96363 \\
\hline
\end{tabular}

$60 \%$ ee

Column: AD-H;

Eluent: $n$-Hexane: $i$-PrOH $=97: 3$;

Flow rate: $1 \mathrm{~mL} / \mathrm{min}$;

Pressure: $4.0 \mathrm{Mpa}$;

Wavelength: $270 \mathrm{~nm}$;

Temperature: $35^{\circ} \mathrm{C}$ 


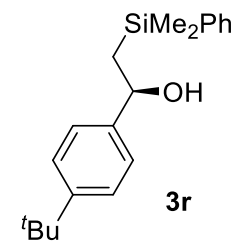

Racemic sample:

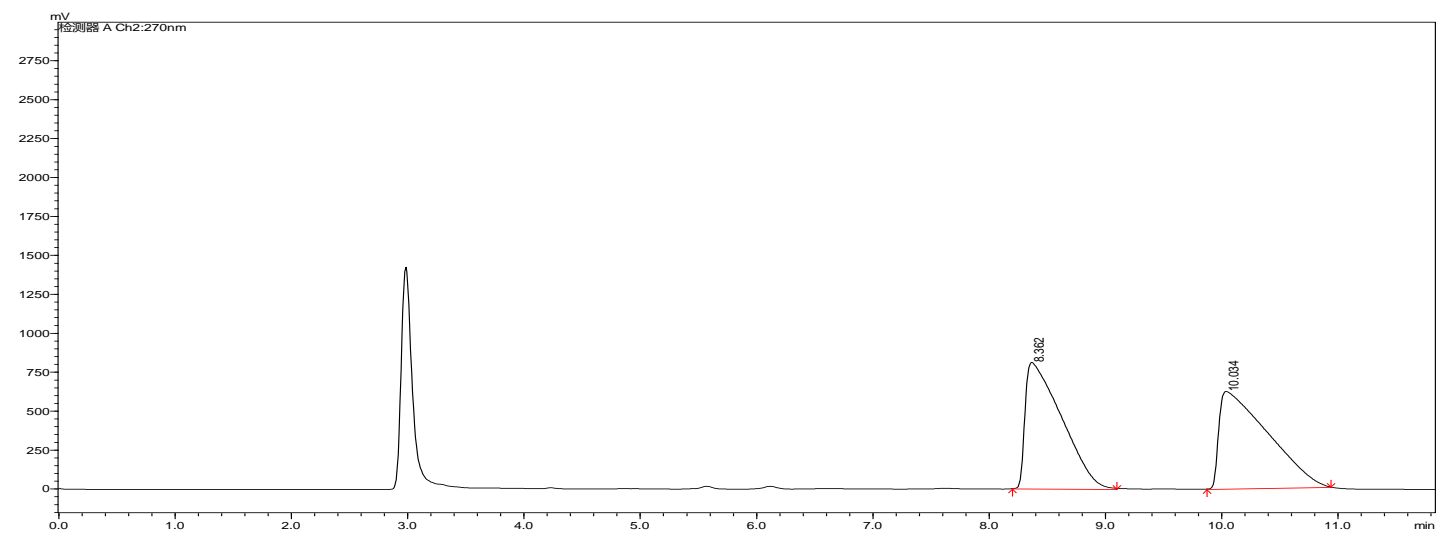

\begin{tabular}{|l|l|l|l|}
\hline Time & Conc. & Area & Height \\
\hline 8.362 & 49.09888 & 17712916 & 812980 \\
\hline 10.034 & 50.60112 & 18363096 & 628508 \\
\hline
\end{tabular}

Enantio-enriched sample:

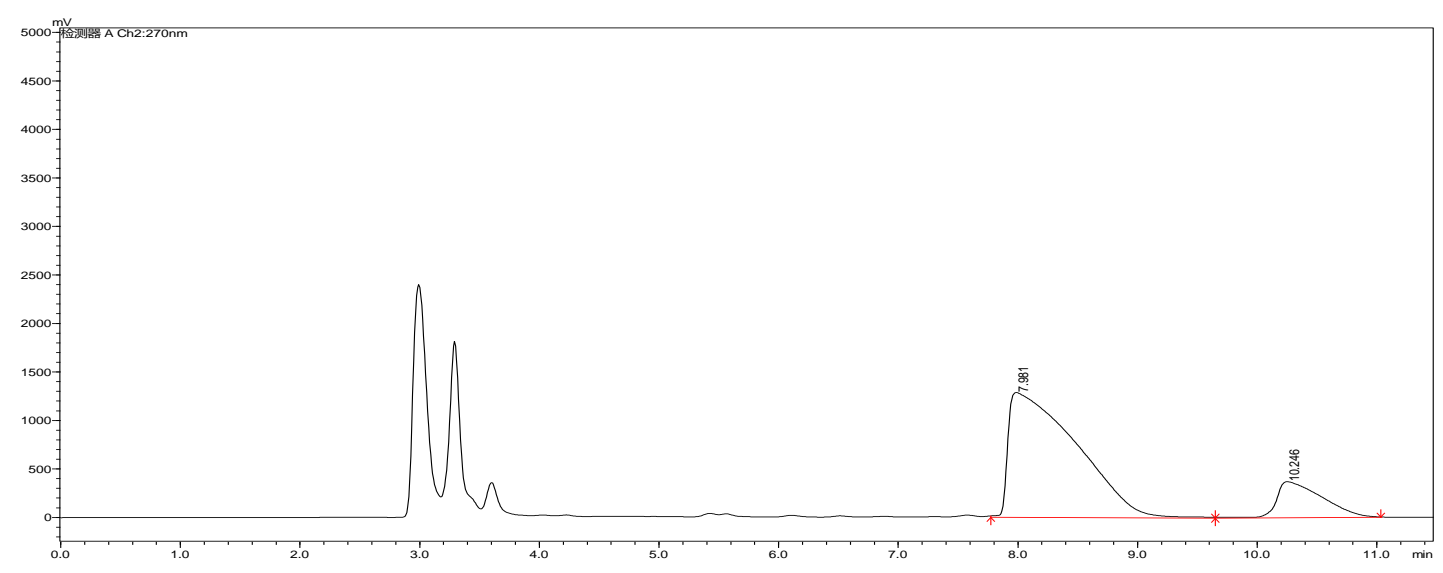

\begin{tabular}{|l|l|l|l|}
\hline Time & Conc. & Area & Height \\
\hline 7.981 & 83.74217 & 51023217 & 1286626 \\
\hline 10.246 & 16.25783 & 9905720 & 372614 \\
\hline
\end{tabular}

$67 \%$ ee

Column: AD-H;

Eluent: $n$-Hexane: $i$-PrOH $=97: 3$;

Flow rate: $1 \mathrm{~mL} / \mathrm{min}$;

Pressure: 4.1 Mpa;

Wavelength: $270 \mathrm{~nm}$;

Temperature: $35^{\circ} \mathrm{C}$ 


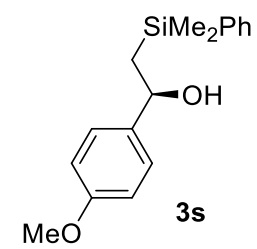

Racemic sample:

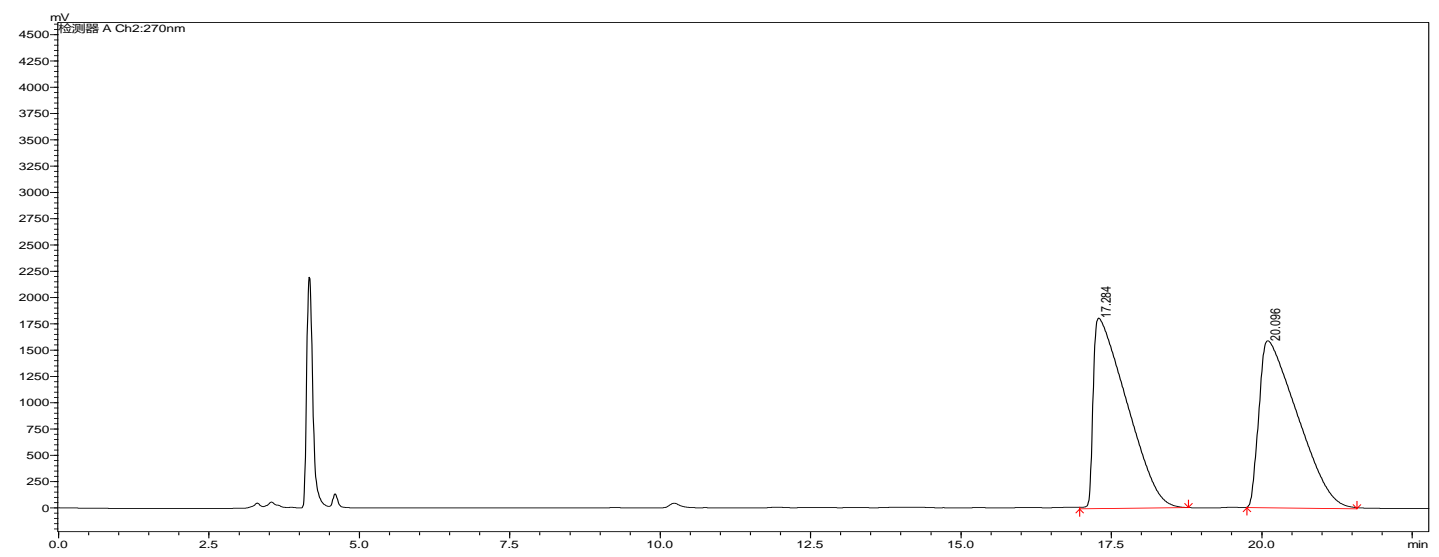

\begin{tabular}{|l|l|l|l|}
\hline Time & Conc. & Area & Height \\
\hline 17.284 & 49.72108 & 69849141 & 1810214 \\
\hline 20.096 & 50.27892 & 70632818 & 1588323 \\
\hline
\end{tabular}

Enantio-enriched sample:

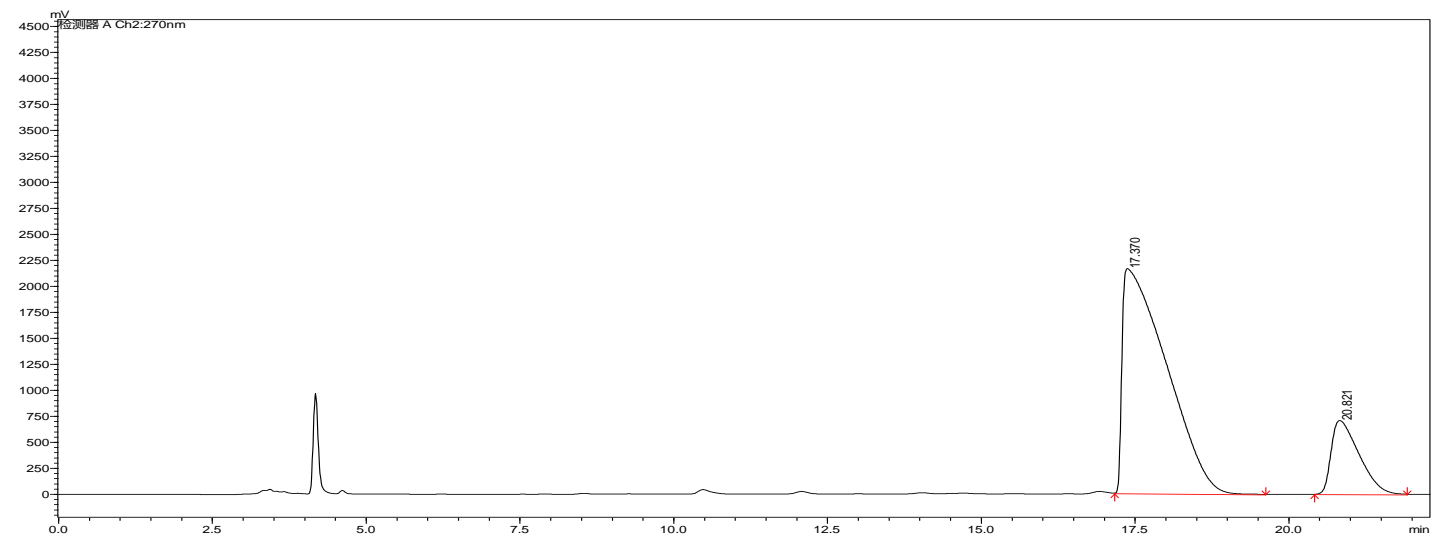

\begin{tabular}{|l|l|l|l|}
\hline Time & Conc. & Area & Height \\
\hline 17.370 & 82.68217 & 108626108 & 2165052 \\
\hline 20.821 & 17.31783 & 22751798 & 713691 \\
\hline
\end{tabular}

$65 \%$ ee

Column: AD-H;

Eluent: $n$-Hexane: $i$-PrOH $=97: 3$;

Flow rate: $1 \mathrm{~mL} / \mathrm{min}$;

Pressure: 4.1 Mpa;

Wavelength: $270 \mathrm{~nm}$;

Temperature: $35^{\circ} \mathrm{C}$ 


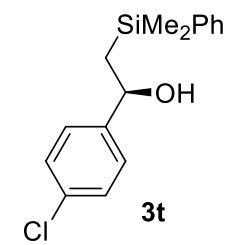

Racemic sample:

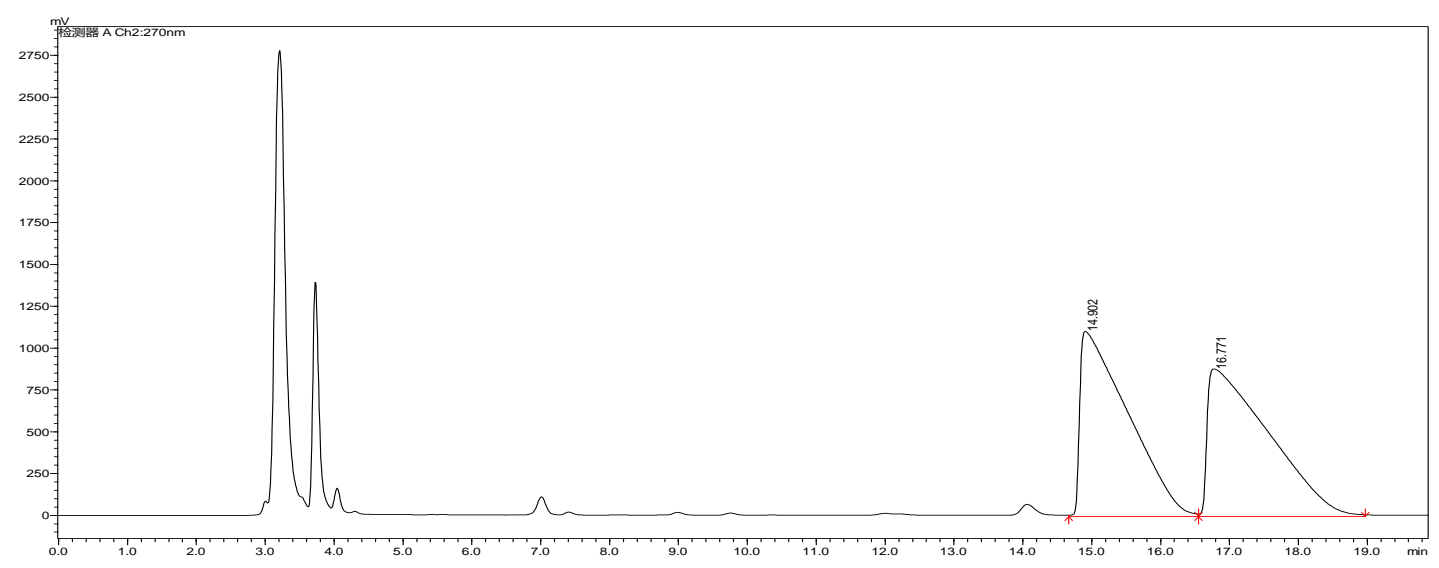

\begin{tabular}{|l|l|l|l|}
\hline Time & Conc. & Area & Height \\
\hline 14.902 & 49.43464 & 53423259 & 1105605 \\
\hline 16.771 & 50.06536 & 54645215 & 881200 \\
\hline
\end{tabular}

Enantio-enriched sample:

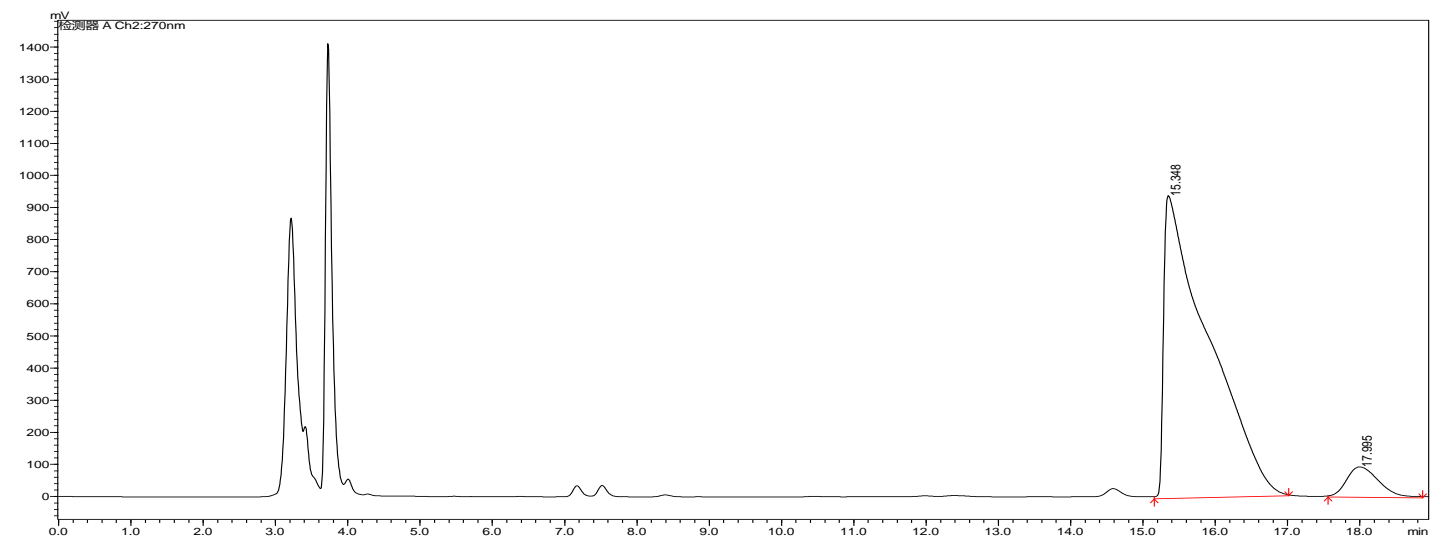

\begin{tabular}{|l|l|l|l|}
\hline Time & Conc. & Area & Height \\
\hline 15.348 & 93.25121 & 40645136 & 942998 \\
\hline 17.995 & 6.74879 & 2941577 & 94204 \\
\hline
\end{tabular}

$87 \%$ ee

Column: AD-H;

Eluent: $n$-Hexane: $i$-PrOH $=98: 2$;

Flow rate: $1 \mathrm{~mL} / \mathrm{min}$;

Pressure: 4.0 Mpa;

Wavelength: $270 \mathrm{~nm}$;

Temperature: $35^{\circ} \mathrm{C}$ 


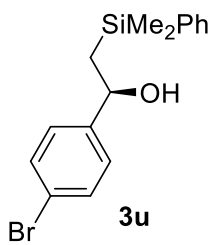

Racemic sample:

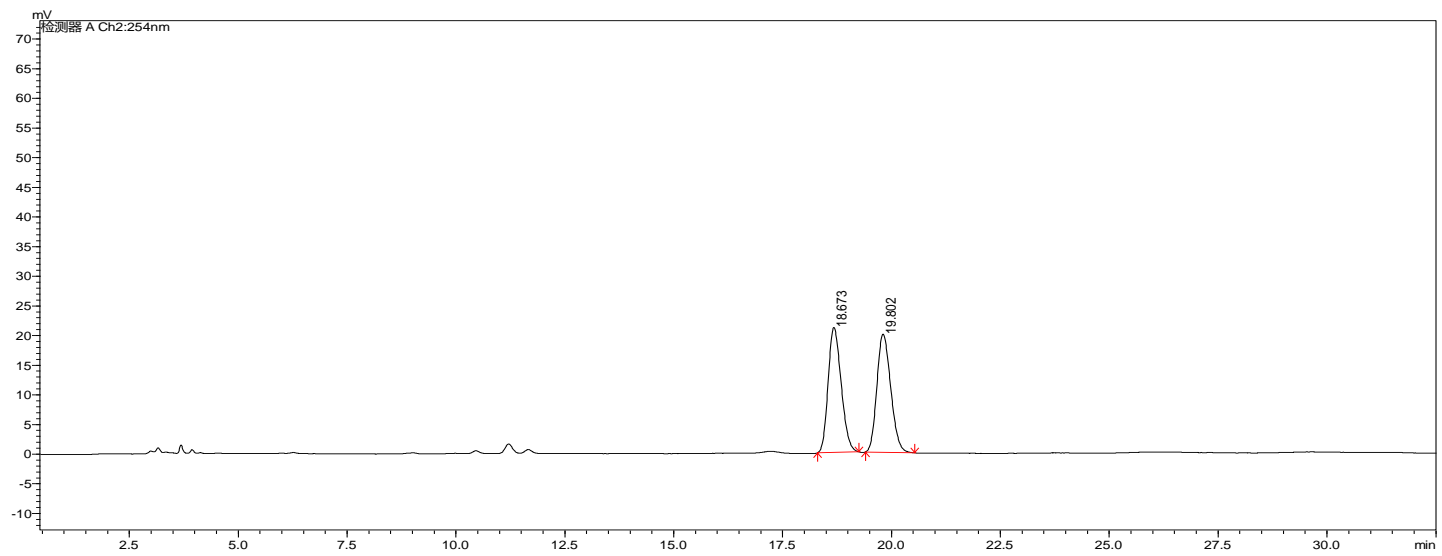

\begin{tabular}{|l|l|l|l|}
\hline Time & Conc. & Area & Height \\
\hline 19.607 & 49.82708 & 1742904 & 73079 \\
\hline 21.350 & 50.17292 & 1697841 & 67183 \\
\hline
\end{tabular}

Enantio-enriched sample:

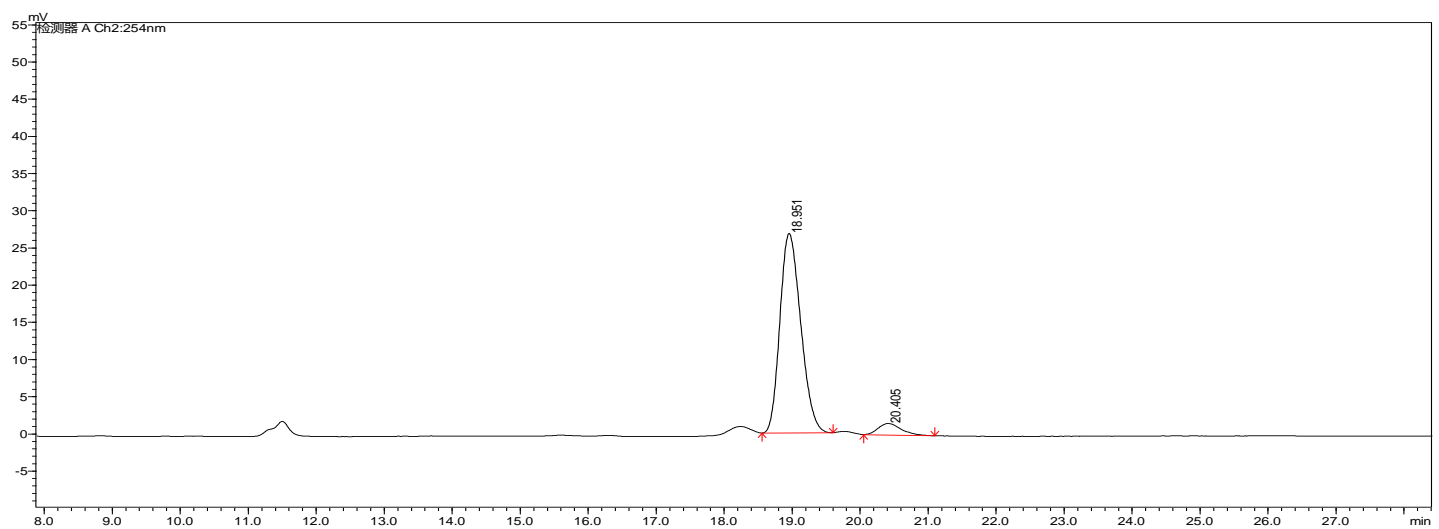

\begin{tabular}{|l|l|l|l|}
\hline Time & Conc. & Area & Height \\
\hline 18.949 & 94.43120 & 781372 & 36661 \\
\hline 20.408 & 5.56880 & 46079 & 2045 \\
\hline
\end{tabular}

$90 \%$ ee

Column: AD-H;

Eluent: $n$-Hexane: $i$-PrOH $=99: 1$;

Flow rate: $1 \mathrm{~mL} / \mathrm{min}$;

Pressure: 4.0 Mpa;

Wavelength: $270 \mathrm{~nm}$;

Temperature: $35^{\circ} \mathrm{C}$ 


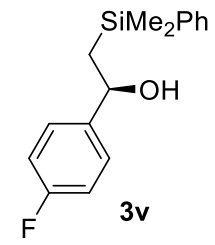

Racemic sample:

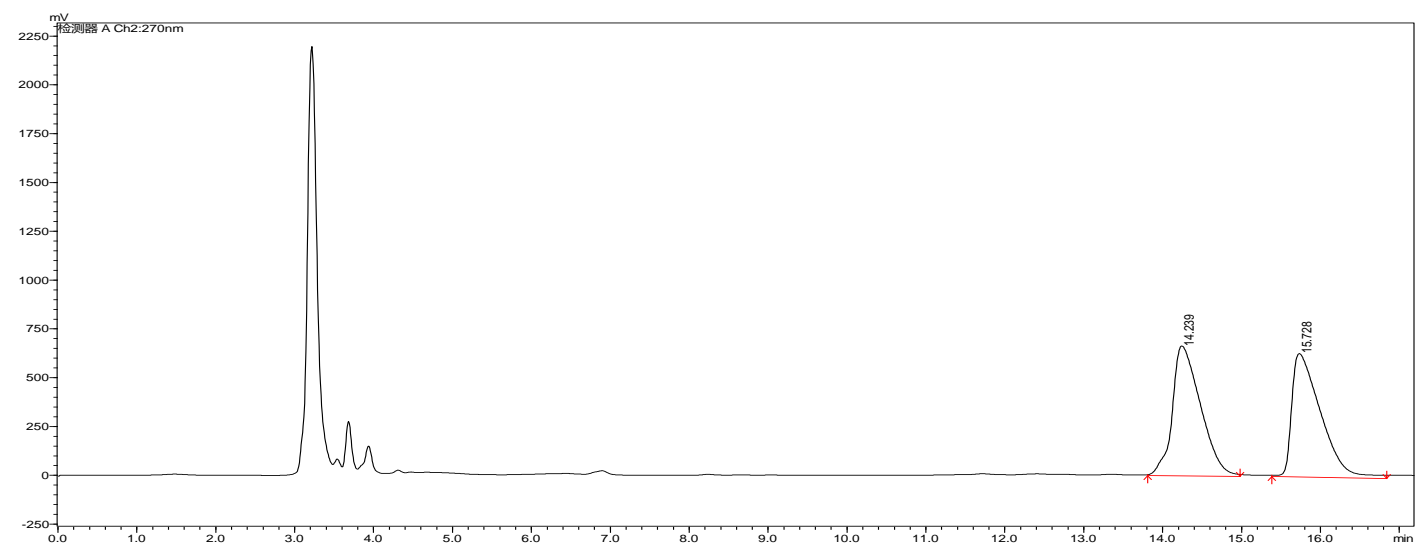

\begin{tabular}{|l|l|l|l|}
\hline Time & Conc. & Area & Height \\
\hline 14.239 & 50.08718 & 16653566 & 665944 \\
\hline 15.728 & 49.51282 & 16137317 & 632252 \\
\hline
\end{tabular}

Enantio-enriched sample:

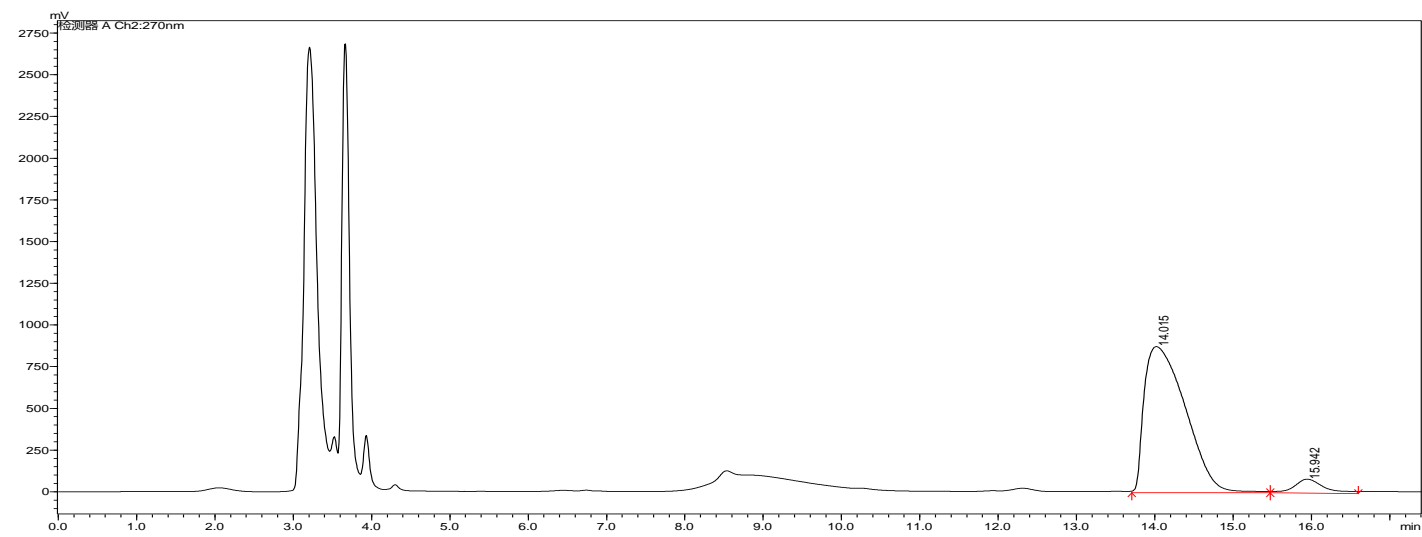

\begin{tabular}{|l|l|l|l|}
\hline Time & Conc. & Area & Height \\
\hline 14.015 & 93.56993 & 31994762 & 875351 \\
\hline 15.942 & 6.43007 & 2198661 & 83166 \\
\hline
\end{tabular}

$87 \%$ ee

Column: AD-H;

Eluent: $n$-Hexane: $i$-PrOH $=98: 2$;

Flow rate: $1 \mathrm{~mL} / \mathrm{min}$;

Pressure: 4.1 Mpa;

Wavelength: $270 \mathrm{~nm}$;

Temperature: $35^{\circ} \mathrm{C}$ 


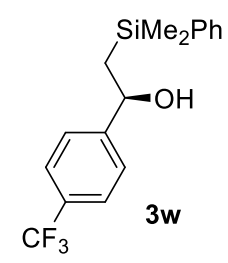

Racemic sample:

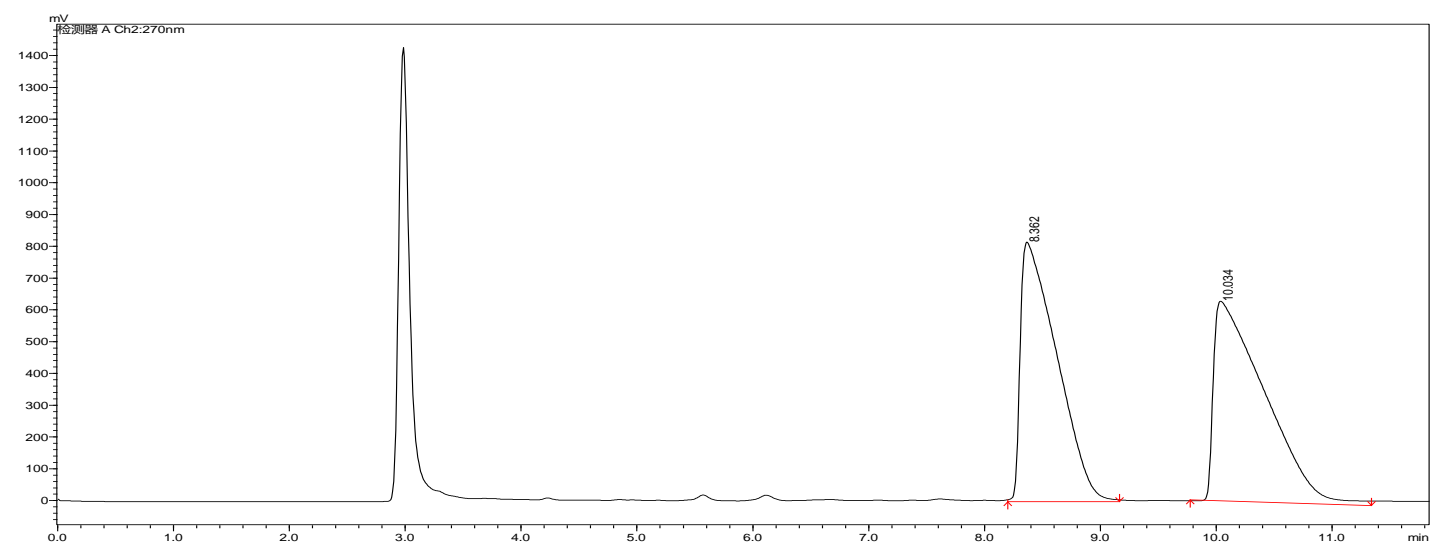

\begin{tabular}{|l|l|l|l|}
\hline Time & Conc. & Area & Height \\
\hline 8.362 & 49.15591 & 17874798 & 816954 \\
\hline 10.034 & 50.84409 & 19243801 & 627721 \\
\hline
\end{tabular}

Enantio-enriched sample:

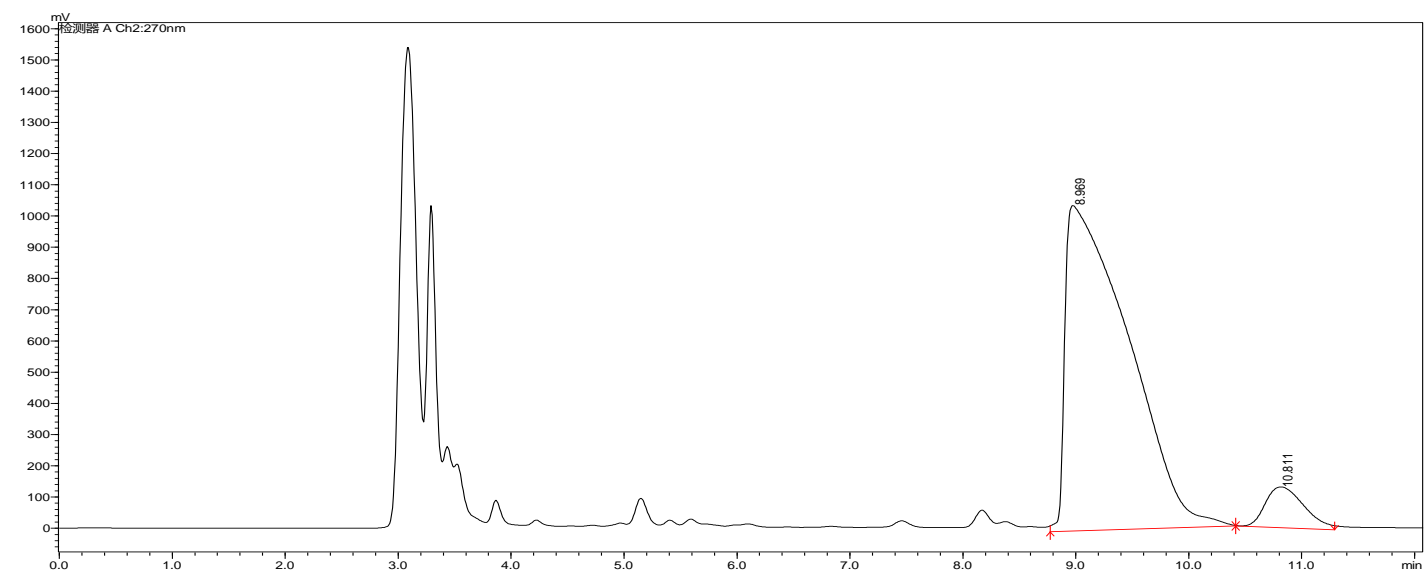

\begin{tabular}{|l|l|l|l|}
\hline Time & Conc. & Area & Height \\
\hline 8.969 & 92.86127 & 40565830 & 1042945 \\
\hline 10.811 & 7.13873 & 3118507 & 130425 \\
\hline
\end{tabular}

$86 \%$ ee

Column: AD-H;

Eluent: $n$-Hexane: $i$-PrOH $=97: 3$;

Flow rate: $1 \mathrm{~mL} / \mathrm{min}$;

Pressure: $4.0 \mathrm{Mpa}$;

Wavelength: $270 \mathrm{~nm}$;

Temperature: $35^{\circ}$ 


\section{Copies of NMR spectra for the key compounds}

${ }^{1} \mathrm{H}$ NMR of $1 \mathbf{a}\left(400 \mathrm{MHZ}, \mathrm{CDCl}_{3}\right)$

ำกำ

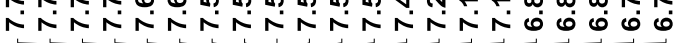
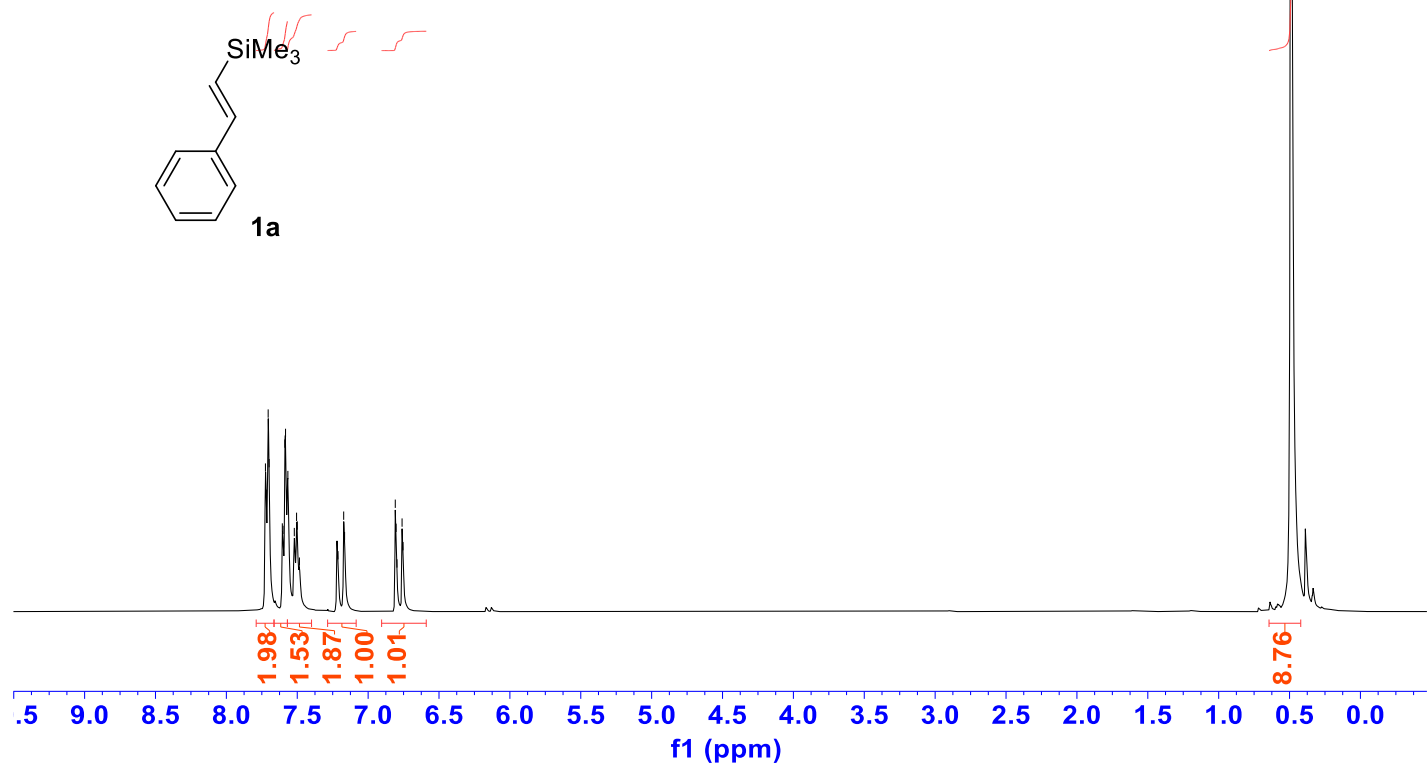

${ }^{13} \mathrm{C}\left\{{ }^{1} \mathrm{H}\right\} \mathrm{NMR}$ of 1a $\left(100 \mathrm{MHZ}, \mathrm{CDCl}_{3}\right)$

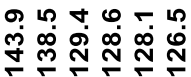

ำำำ

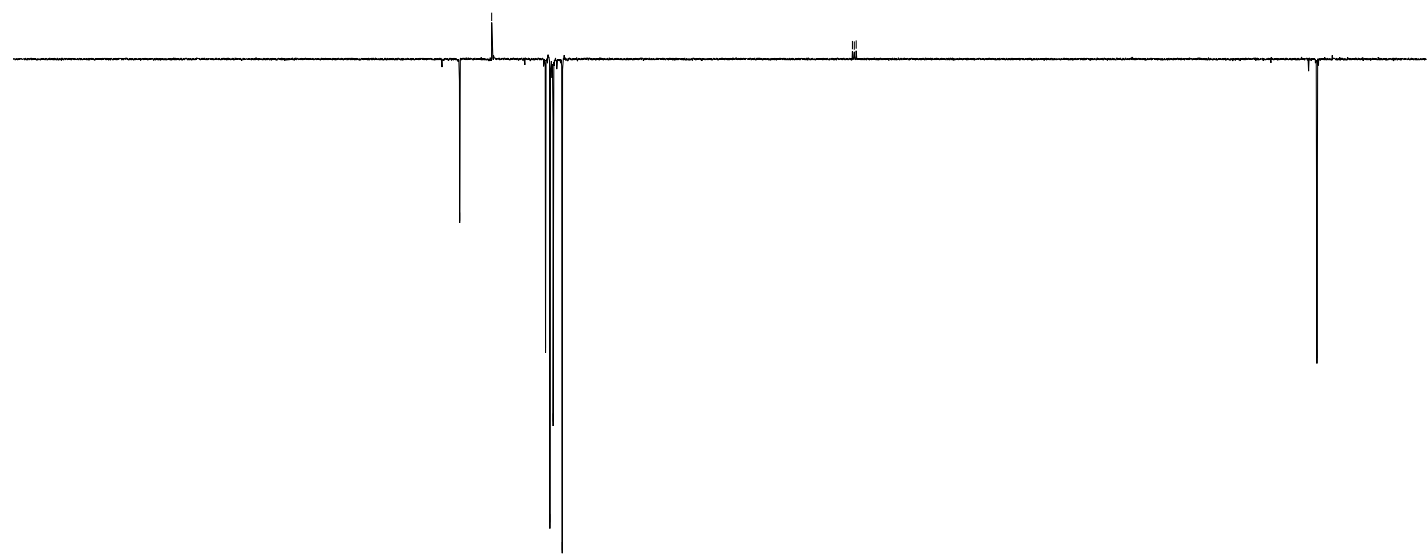

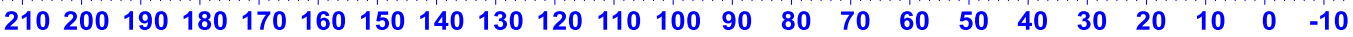
f1 (ppm) 
${ }^{1} \mathrm{H} \mathrm{NMR}$ of $\mathbf{1 b}\left(400 \mathrm{MHZ}, \mathrm{CDCl}_{3}\right)$

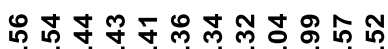

NNNNNNNobo

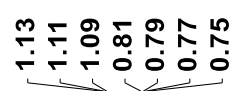

$\sum_{1 b}^{S_{1 b}}$

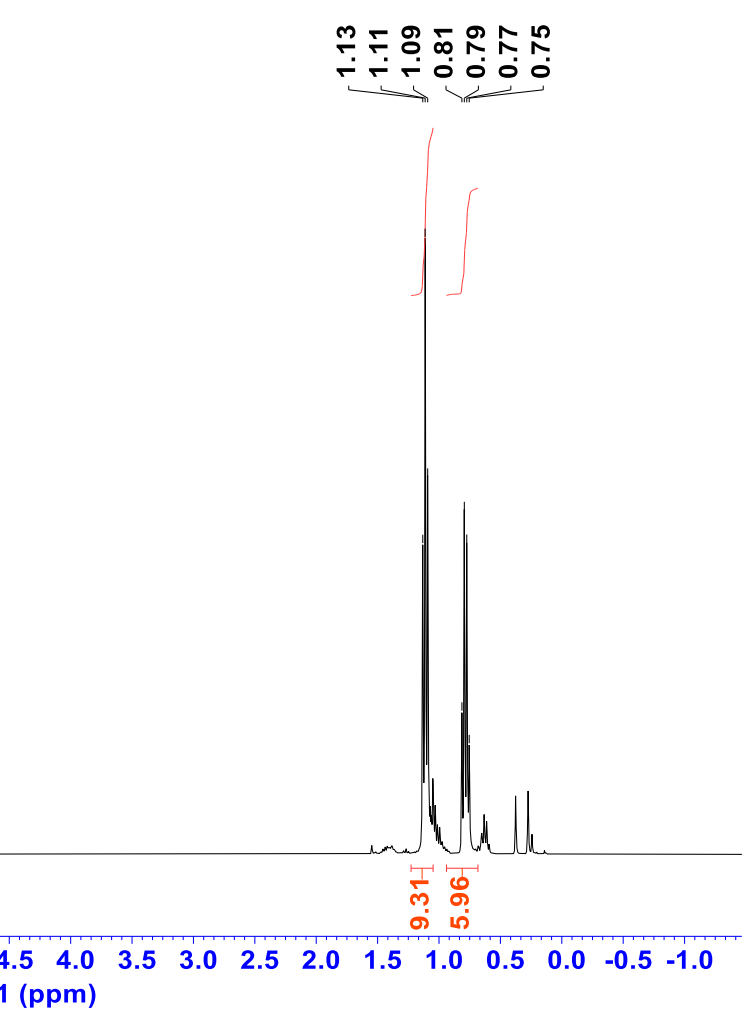

${ }^{13} \mathrm{C}\left\{{ }^{1} \mathrm{H}\right\}$ NMR of $\mathbf{1 b}\left(100 \mathrm{MHZ}, \mathrm{CDCl}_{3}\right)$

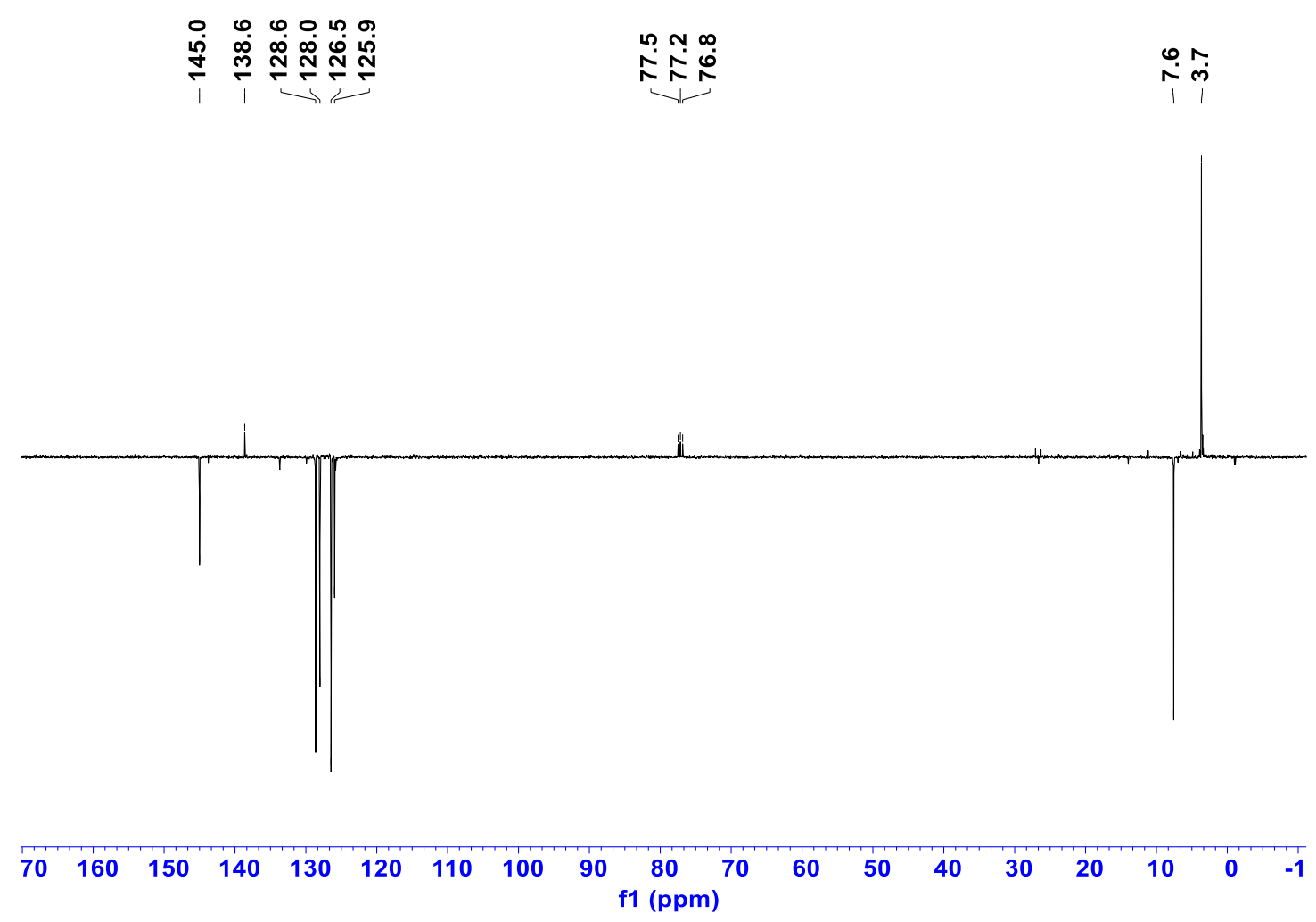

SI-33 
${ }^{1} \mathrm{H}$ NMR of $1 \mathrm{c}\left(400 \mathrm{MHZ}, \mathrm{CDCl}_{3}\right)$

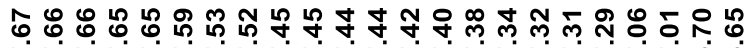

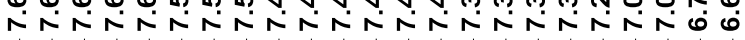
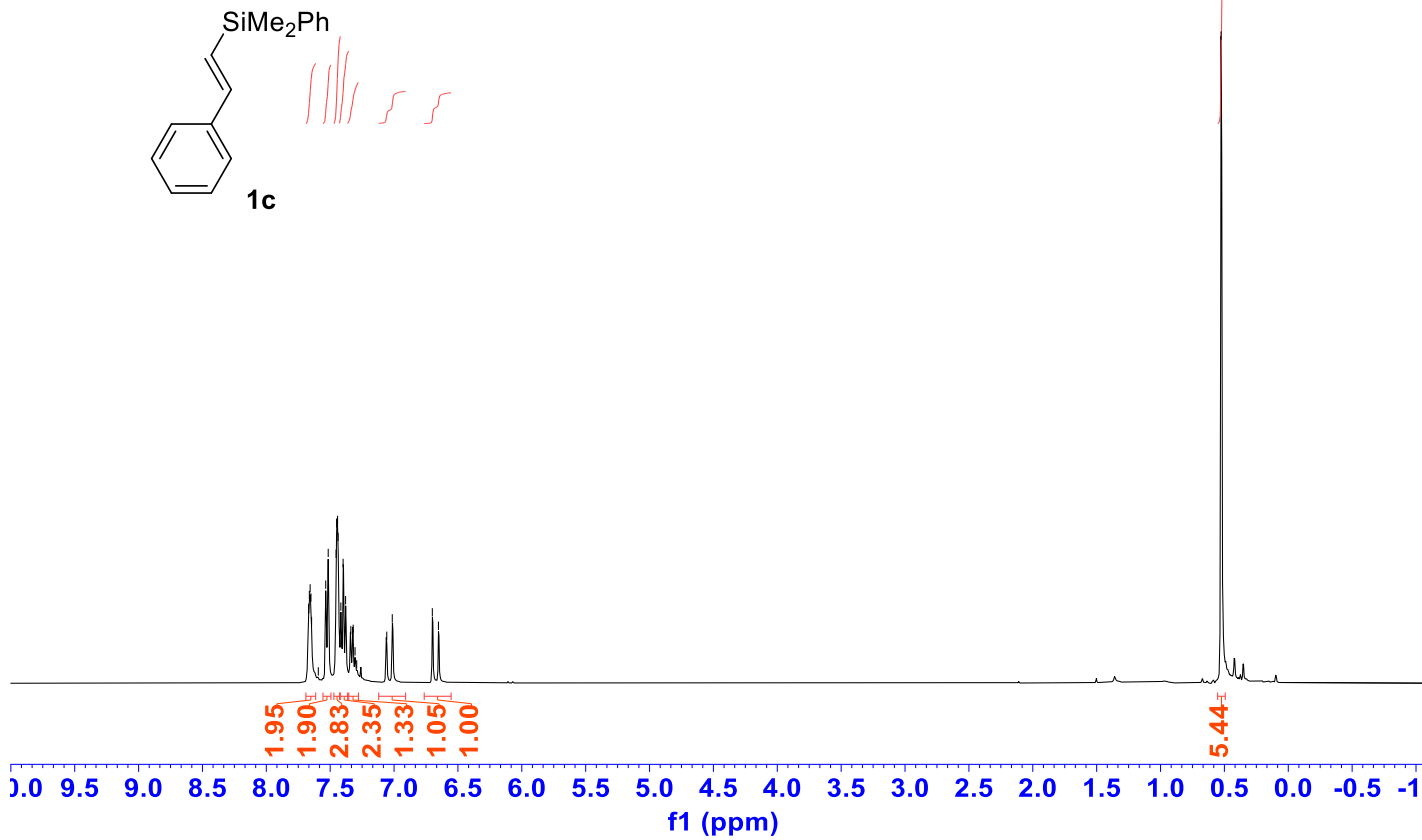

${ }^{13} \mathrm{C}\left\{{ }^{1} \mathrm{H}\right\}$ NMR of $1 \mathbf{c}\left(100 \mathrm{MHZ}, \mathrm{CDCl}_{3}\right)$

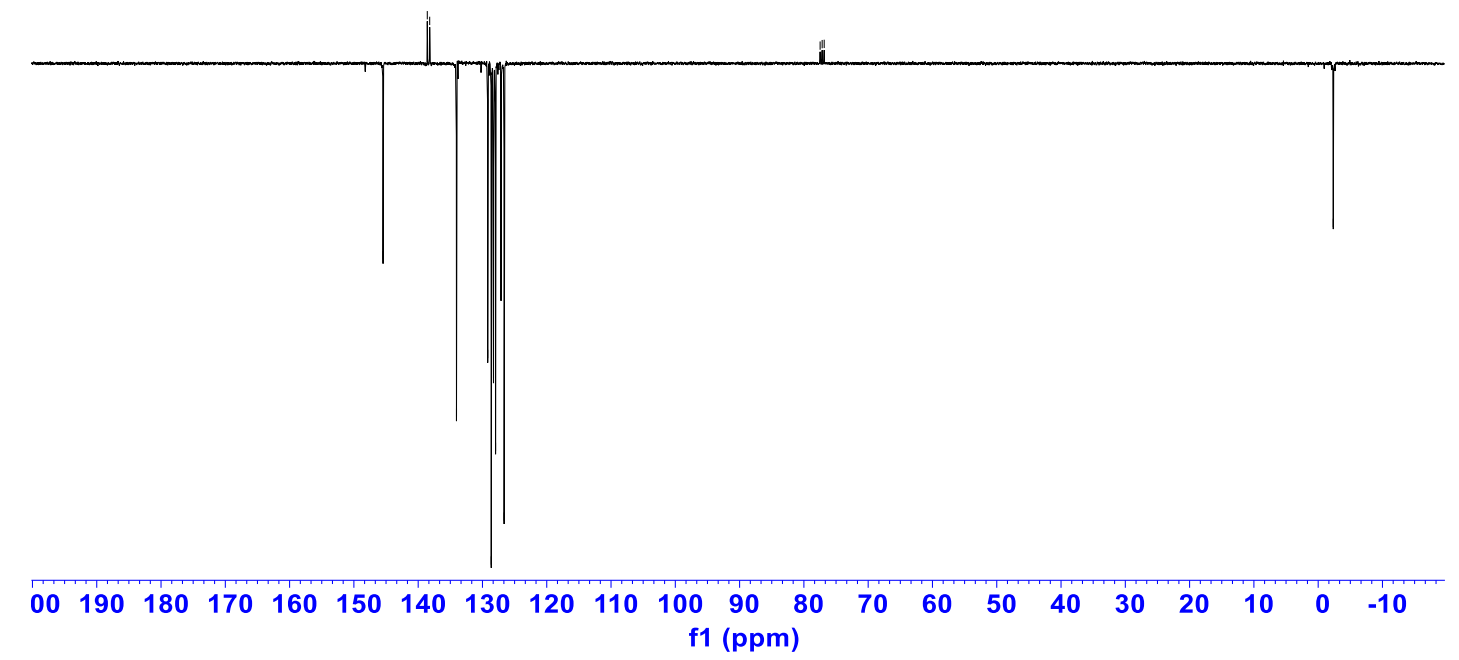


${ }^{1} \mathrm{H} \mathrm{NMR}$ of $\mathbf{1 d}\left(400 \mathrm{MHZ}, \mathrm{CDCl}_{3}\right)$

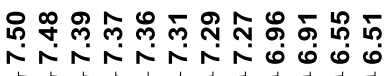
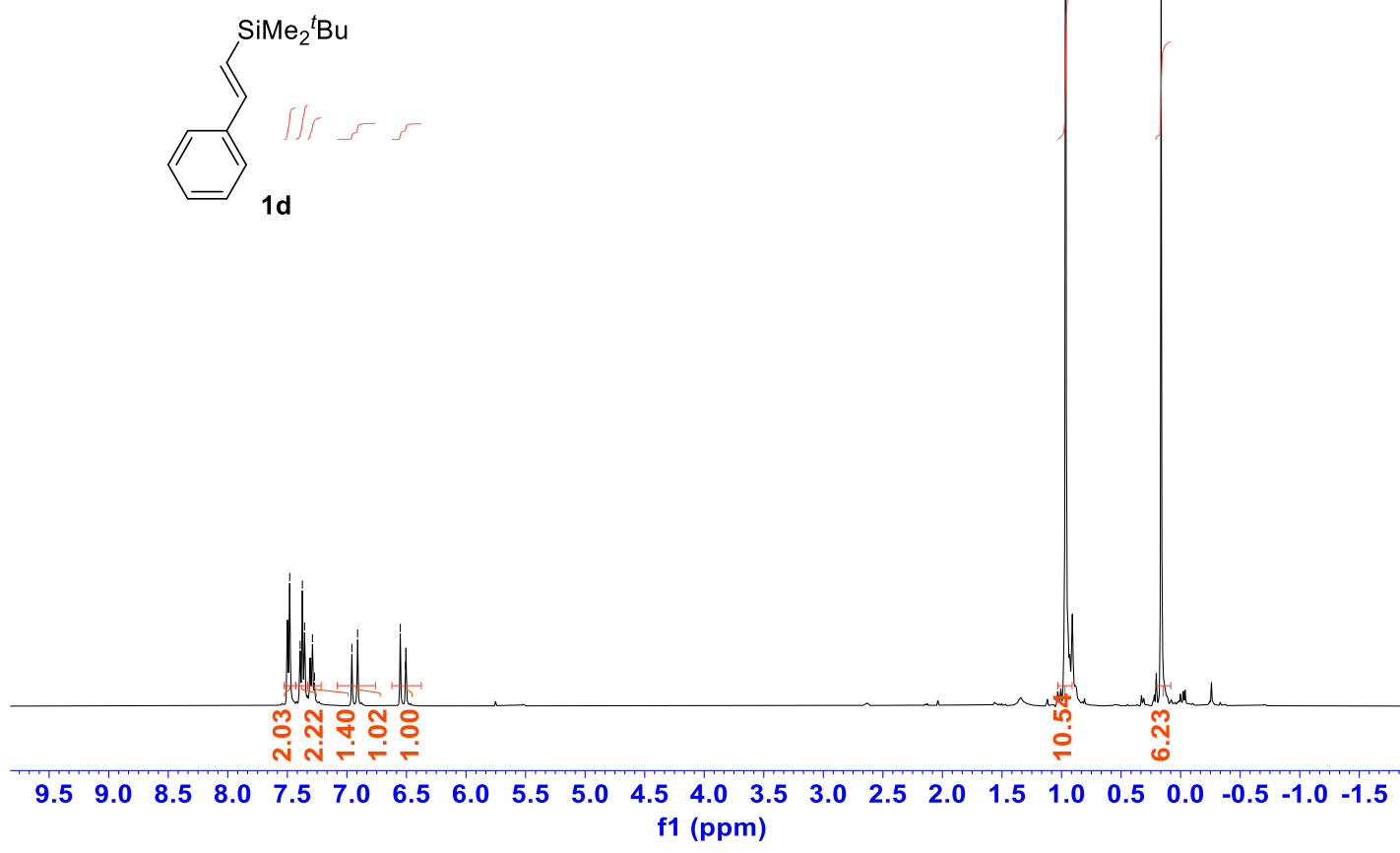

${ }^{13} \mathrm{C}\left\{{ }^{1} \mathrm{H}\right\}$ NMR of $1 \mathbf{d}\left(100 \mathrm{MHZ}, \mathrm{CDCl}_{3}\right)$

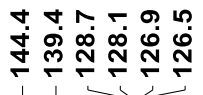

กำ゚ฺ

ஸे

พุ

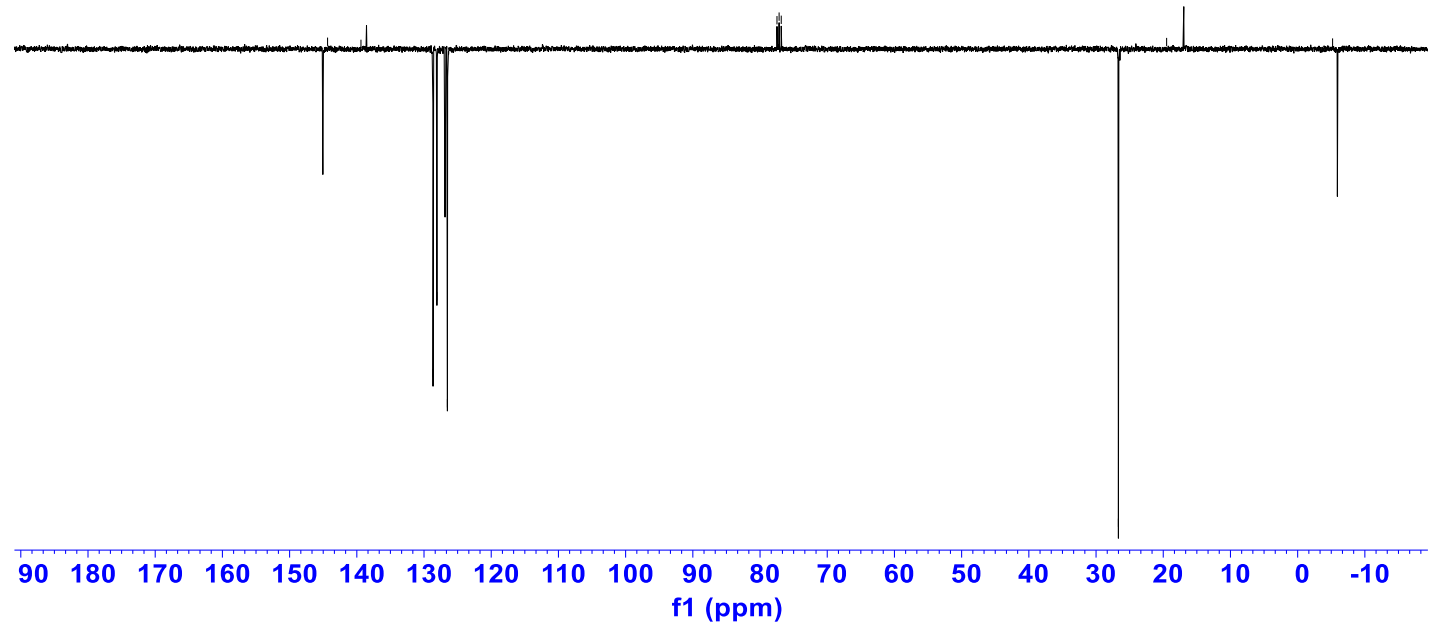


${ }^{1} \mathrm{H}$ NMR of $1 \mathrm{e}\left(400 \mathrm{MHZ}, \mathrm{CDCl}_{3}\right)$

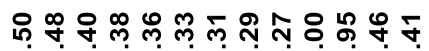

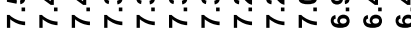

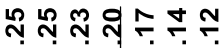
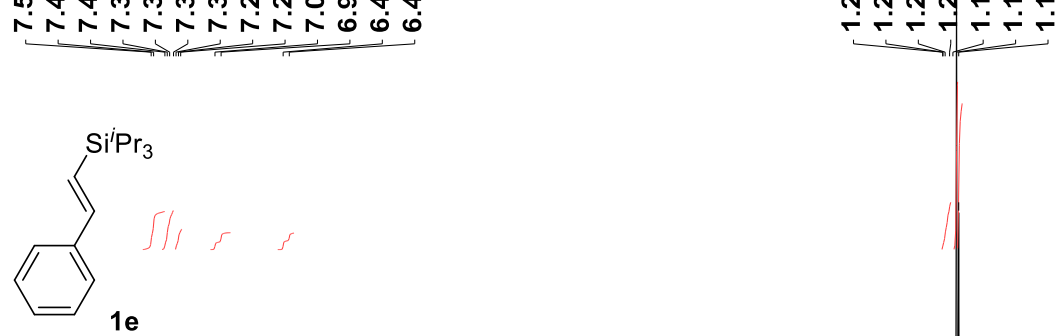

-

我

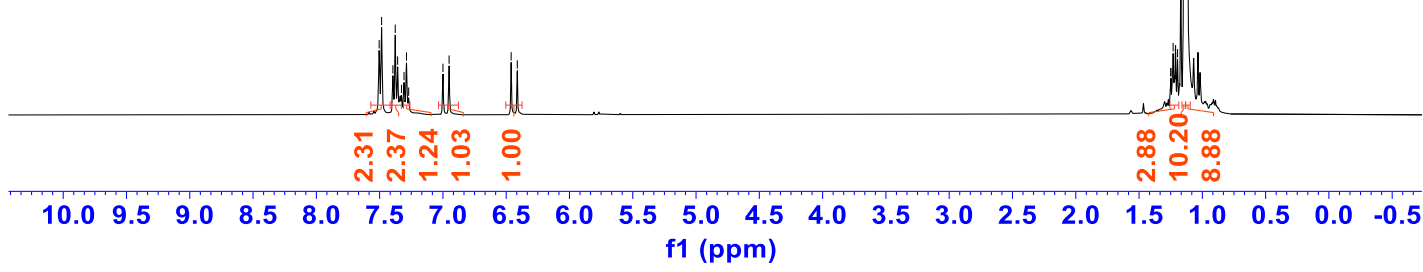

${ }^{13} \mathrm{C}\left\{{ }^{1} \mathrm{H}\right\} \mathrm{NMR}$ of $1 \mathrm{e}\left(100 \mathrm{MHZ}, \mathrm{CDCl}_{3}\right)$

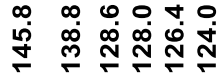

م

$\stackrel{\infty}{\infty} \underset{1}{\circ}$

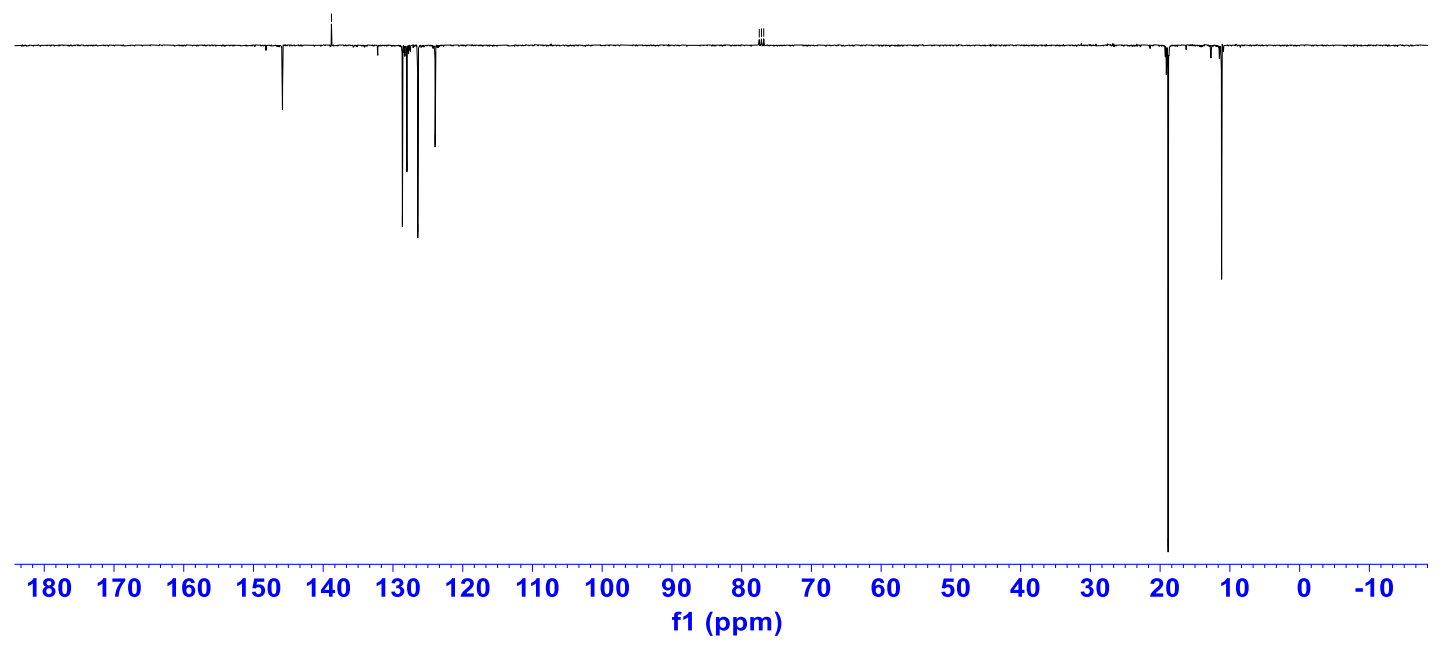


${ }^{1} \mathrm{H}$ NMR of $\mathbf{1 f}\left(400 \mathrm{MHZ}, \mathrm{CDCl}_{3}\right)$

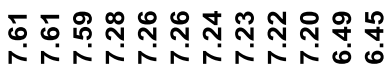

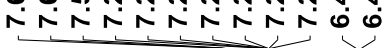

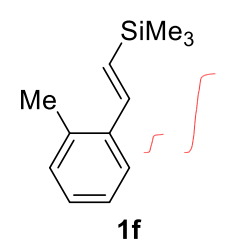

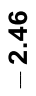

$1 f$

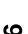

$+2$
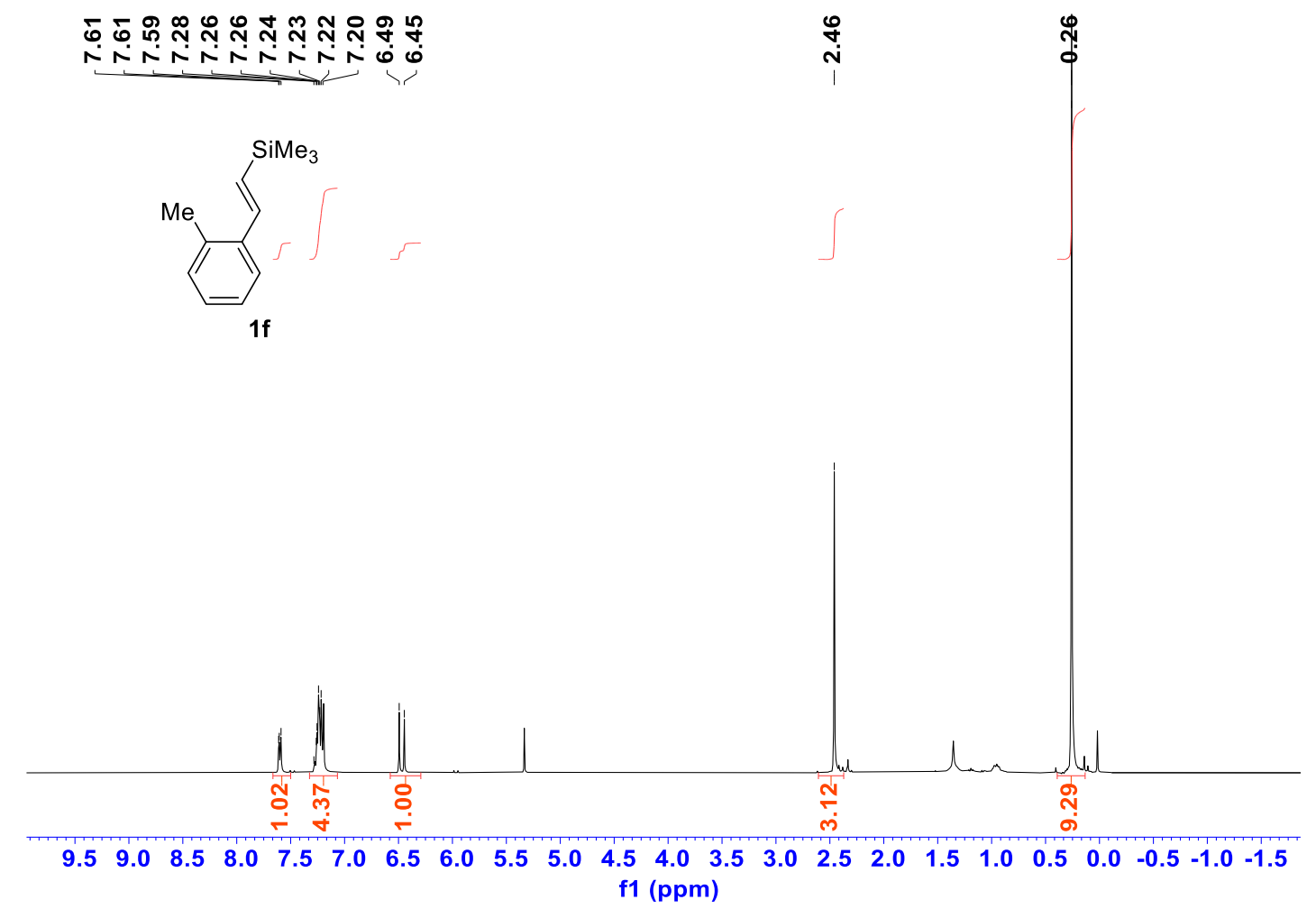

${ }^{13} \mathrm{C}\left\{{ }^{1} \mathrm{H}\right\}$ NMR of $\mathbf{1 f}\left(100 \mathrm{MHZ}, \mathrm{CDCl}_{3}\right)$

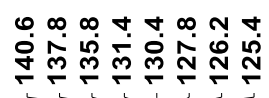

กิ๊

$\stackrel{5}{i} \quad \frac{0}{i}$

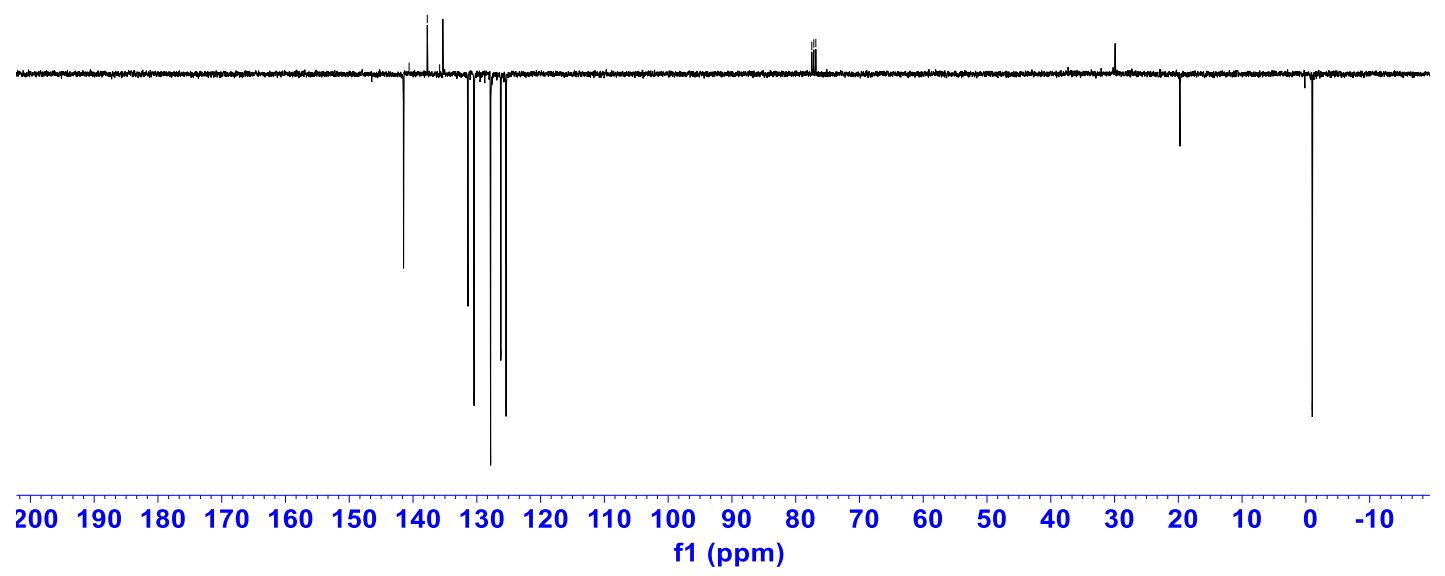


${ }^{1} \mathrm{H}$ NMR of $1 \mathrm{~g}\left(400 \mathrm{MHZ}, \mathrm{CDCl}_{3}\right)$

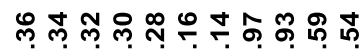

inivisitiogo

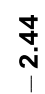

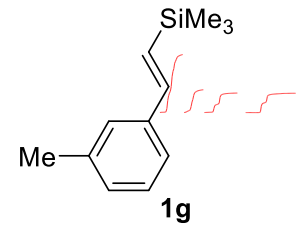

$1 \mathrm{~g}$

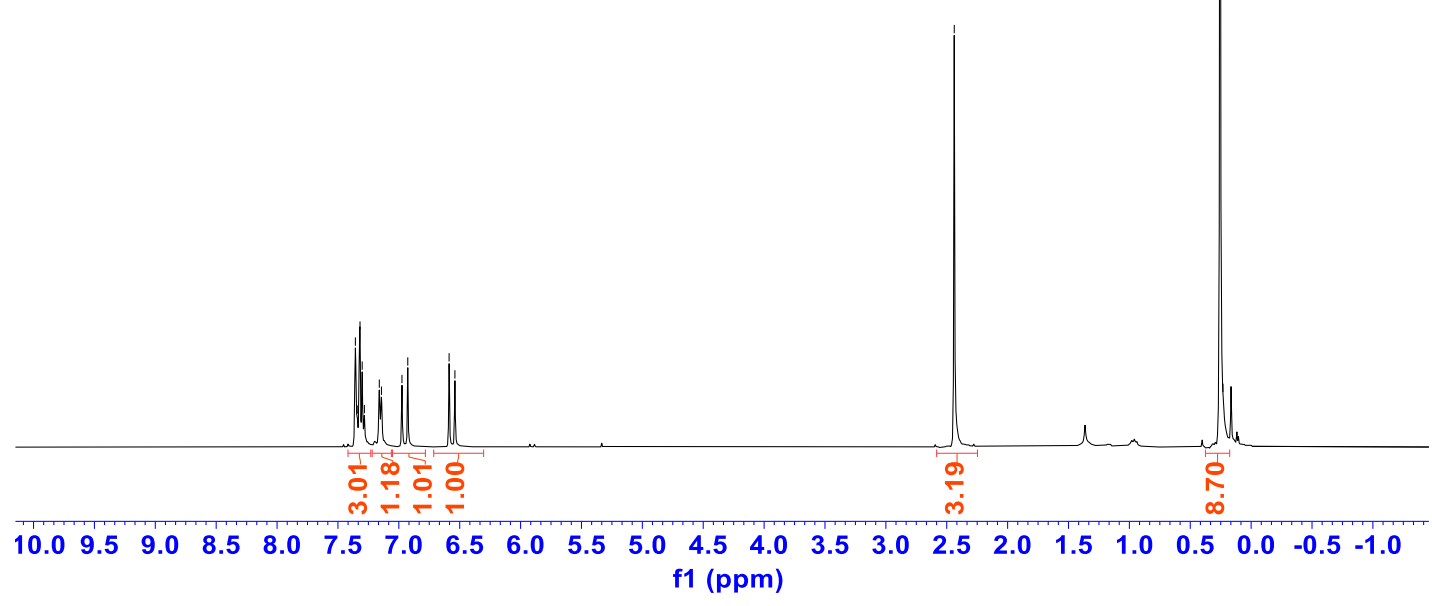

${ }^{13} \mathrm{C}\left\{{ }^{1} \mathrm{H}\right\}$ NMR of $\mathbf{1 g}\left(100 \mathrm{MHZ}, \mathrm{CDCl}_{3}\right)$

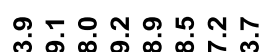

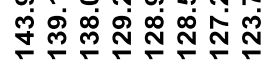

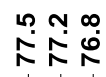

$\stackrel{\stackrel{+}{r}}{i}$

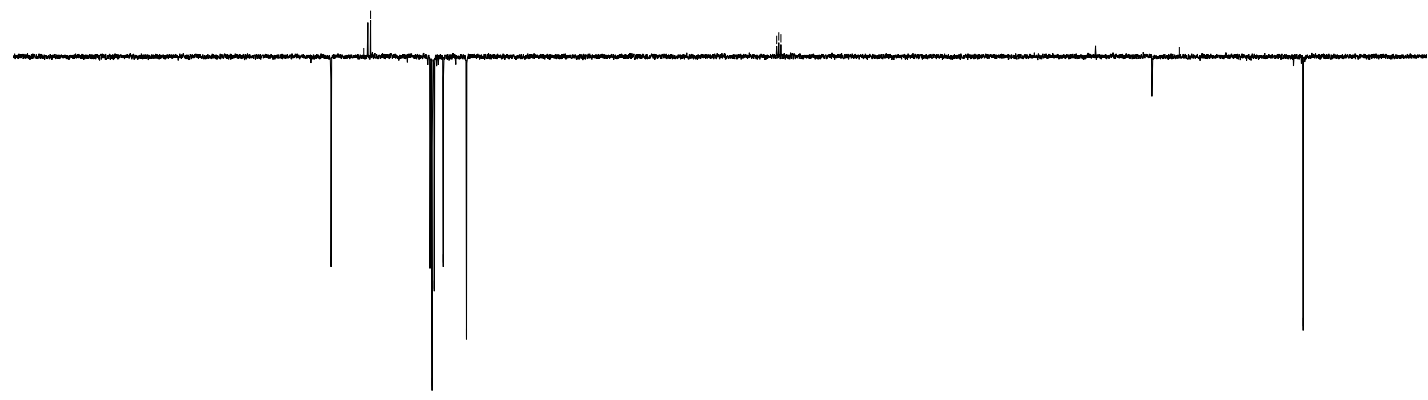

\begin{tabular}{|lllllllllllllllllllll}
90 & 180 & 170 & 160 & 150 & 140 & 130 & 120 & 110 & 100 & 90 & 80 & 70 & 60 & 50 & 40 & 30 & 20 & 10 & 0 & -10
\end{tabular} f1 (ppm) 
${ }^{1} \mathrm{H} \mathrm{NMR}$ of $\mathbf{1 h}\left(400 \mathrm{MHZ}, \mathrm{CDCl}_{3}\right)$

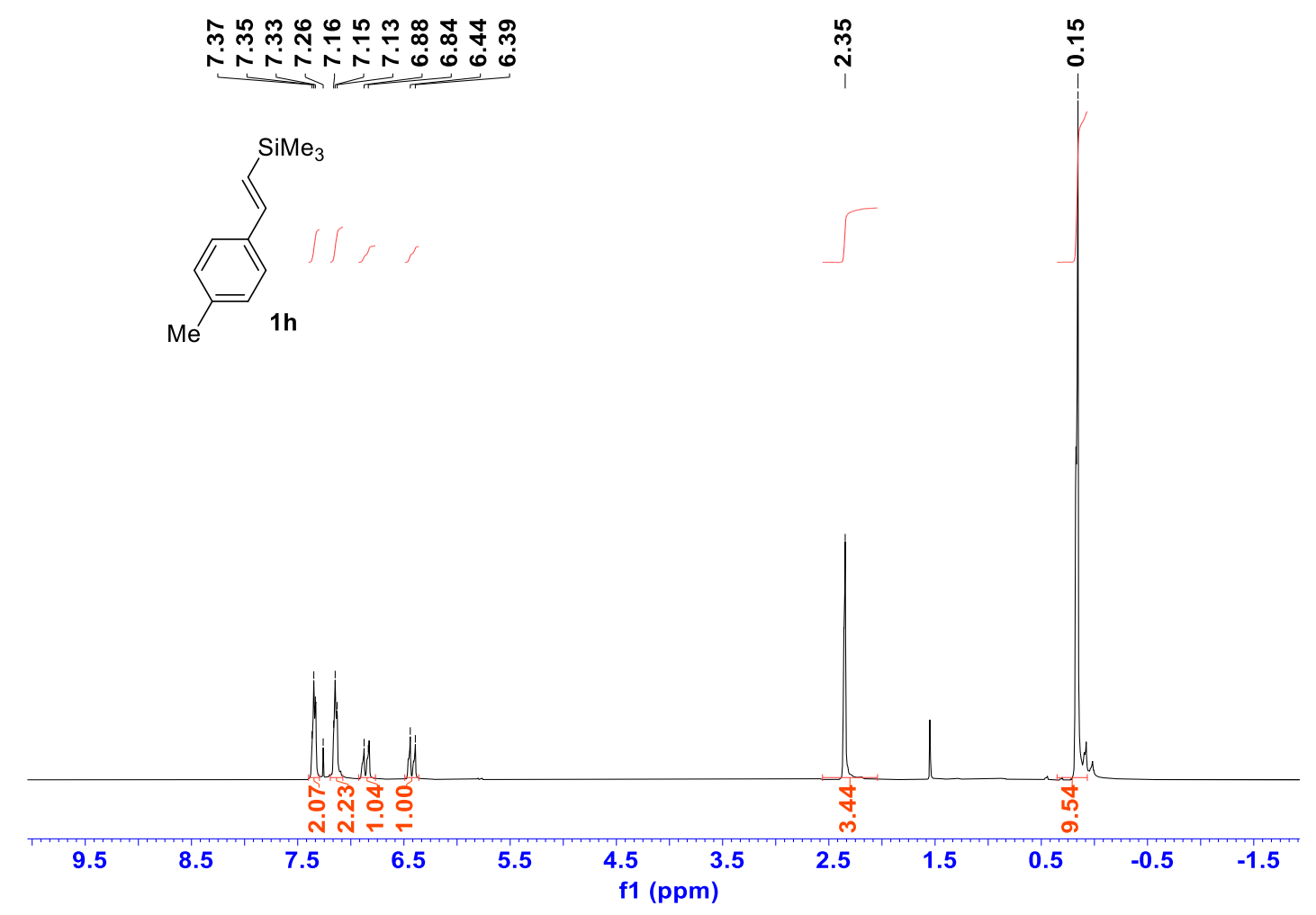

${ }^{13} \mathrm{C}\left\{{ }^{1} \mathrm{H}\right\} \mathrm{NMR}$ of $\mathbf{1 h}\left(100 \mathrm{MHZ}, \mathrm{CDCl}_{3}\right)$

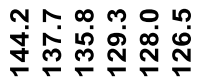

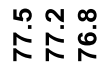

$\stackrel{m}{\sim} \quad \hat{i}$

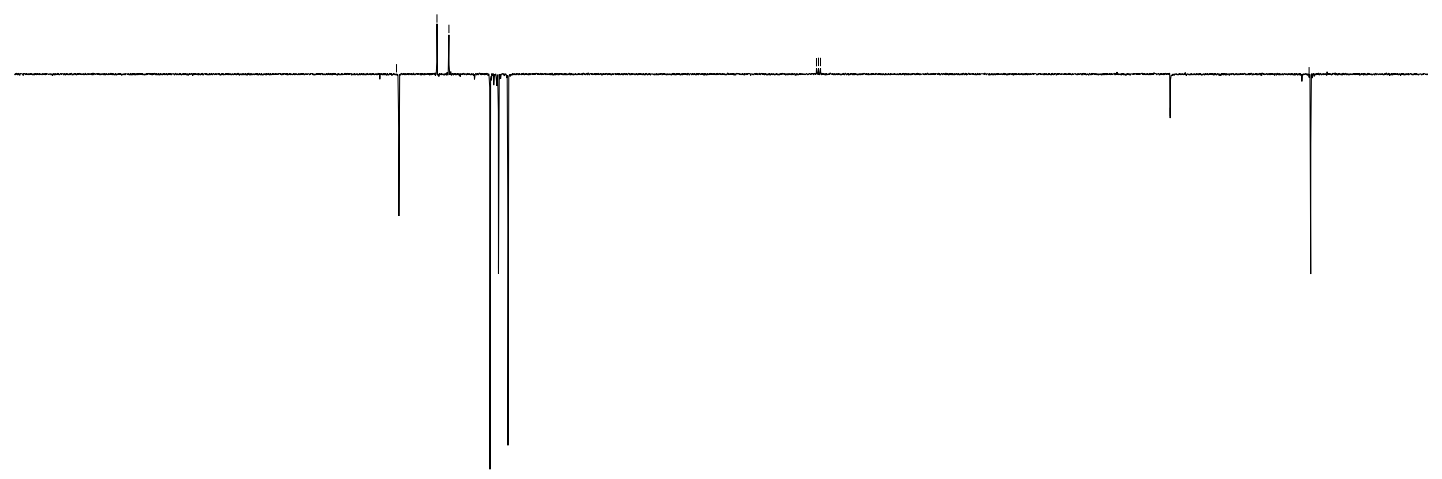

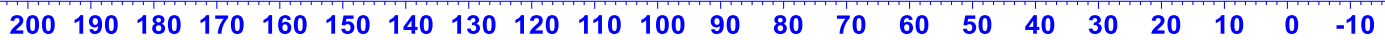
f1 (ppm) 
${ }^{1} \mathrm{H}$ NMR of $1 \mathbf{i}\left(400 \mathrm{MHZ}, \mathrm{CDCl}_{3}\right)$

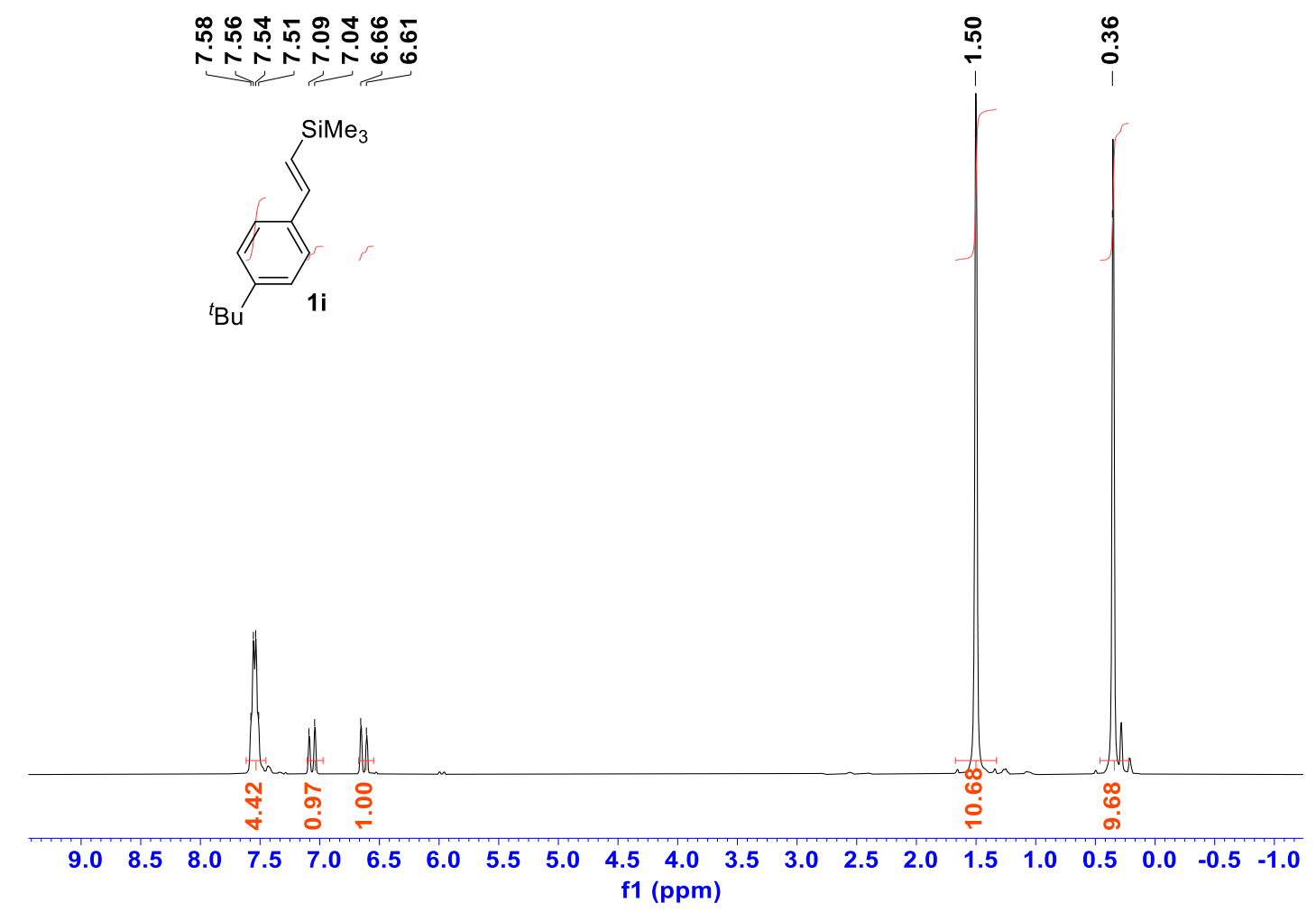

${ }^{13} \mathrm{C}\left\{{ }^{1} \mathrm{H}\right\}$ NMR of $1 \mathbf{i}\left(100 \mathrm{MHZ}, \mathrm{CDCl}_{3}\right)$

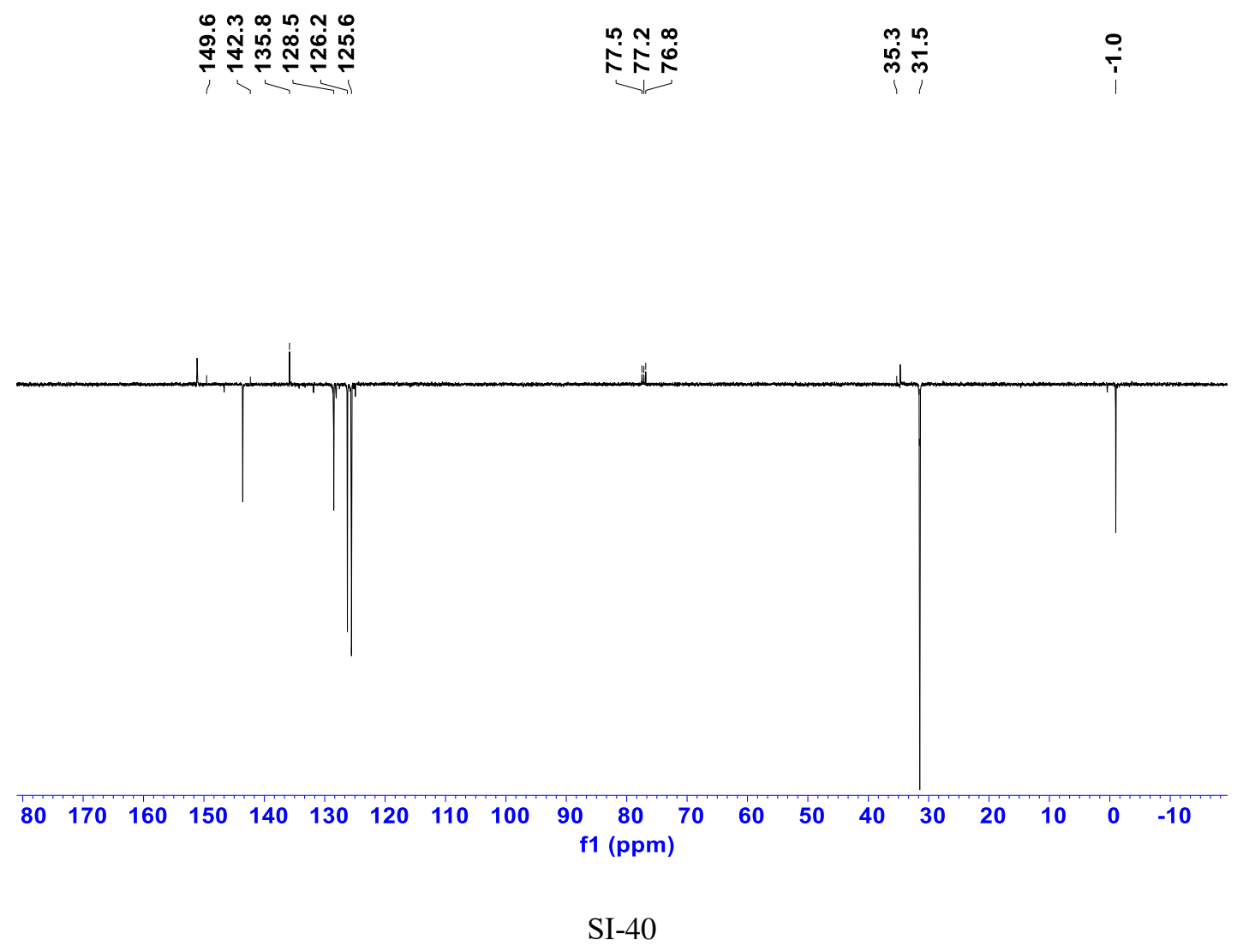


${ }^{1} \mathrm{H}$ NMR of $\mathbf{1 j}\left(400 \mathrm{MHZ}, \mathrm{CDCl}_{3}\right)$

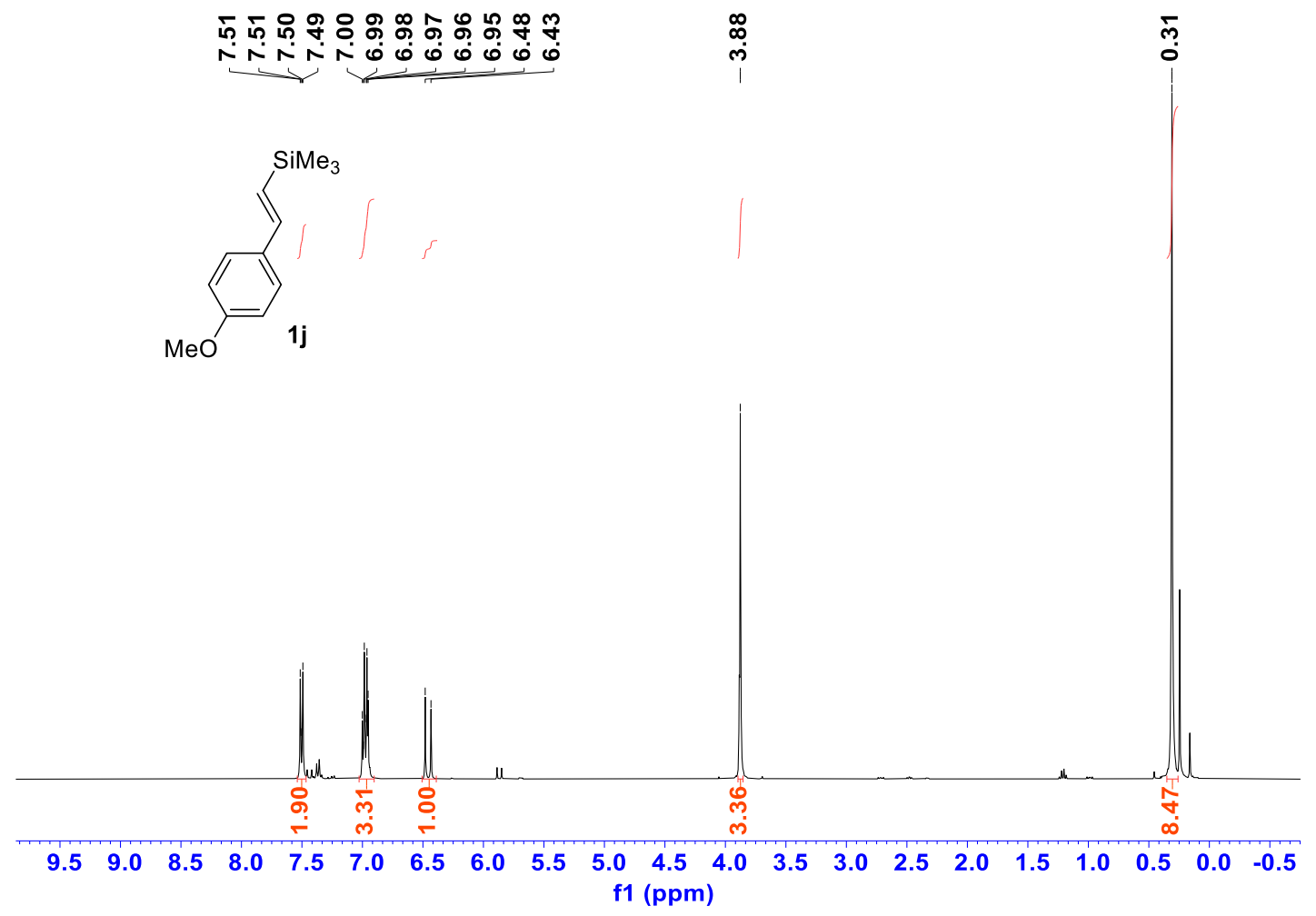

${ }^{13} \mathrm{C}\left\{{ }^{1} \mathrm{H}\right\}$ NMR of $\mathbf{1 j}\left(100 \mathrm{MHZ}, \mathrm{CDCl}_{3}\right)$

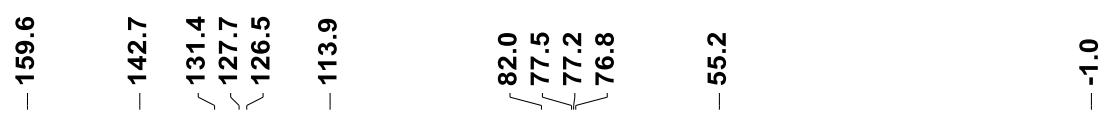

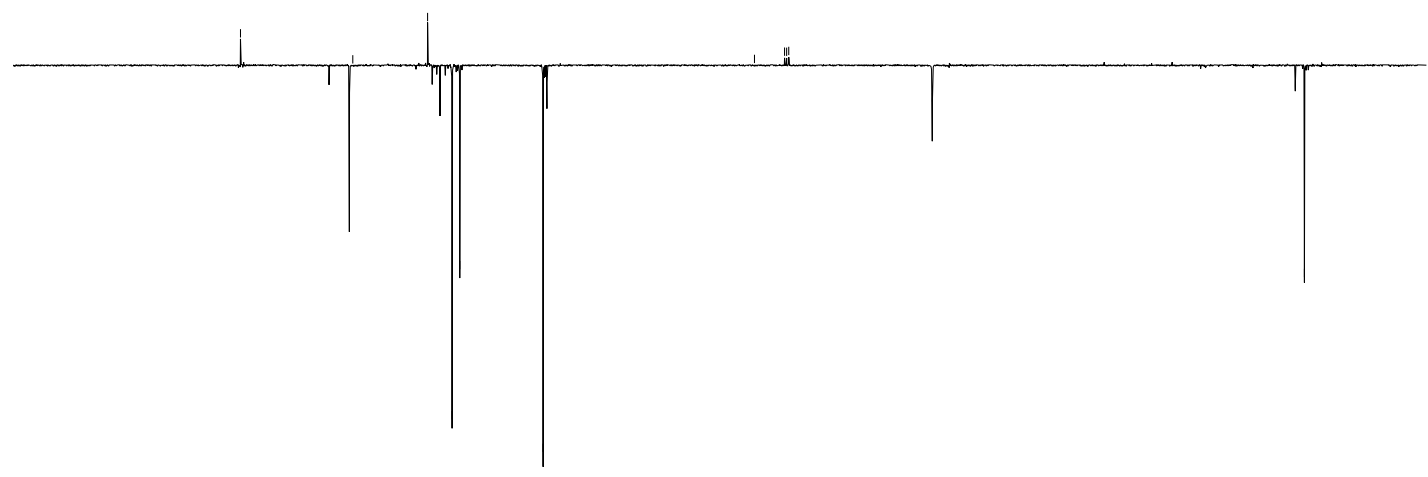

$\begin{array}{lllllllllllllllllllll}190 & 180 & 170 & 160 & 150 & 140 & 130 & 120 & 110 & 100 & 90 & 80 & 70 & 60 & 50 & 40 & 30 & 20 & 10 & 0 & -10\end{array}$ f1 (ppm) 
${ }^{1} \mathrm{H}$ NMR of $1 \mathbf{k}\left(400 \mathrm{MHZ}, \mathrm{CDCl}_{3}\right)$

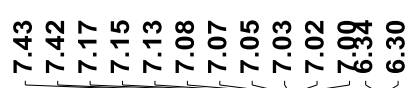

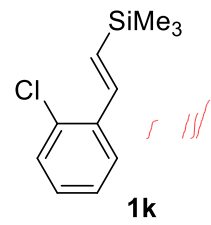

$1 \mathrm{k}$

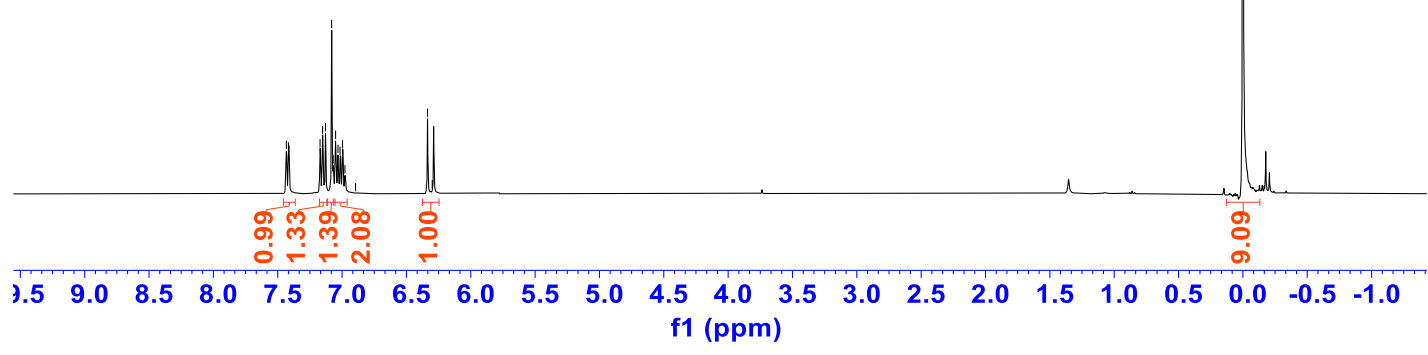

${ }^{13} \mathrm{C}\left\{{ }^{1} \mathrm{H}\right\}$ NMR of $1 \mathbf{k}\left(100 \mathrm{MHZ}, \mathrm{CDCl}_{3}\right)$

ํำ

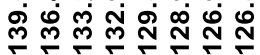

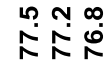

ז

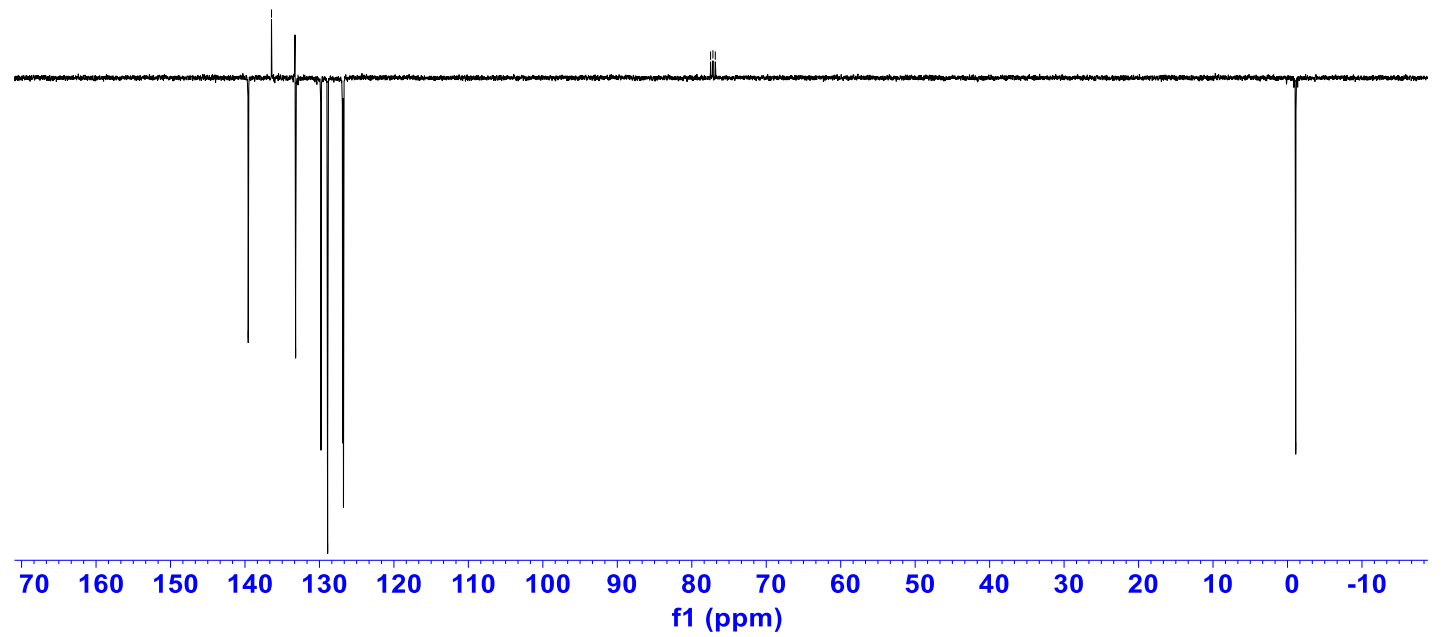


${ }^{1} \mathrm{H}$ NMR of 11 (400 MHZ, $\left.\mathrm{CDCl}_{3}\right)$

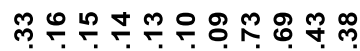

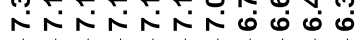

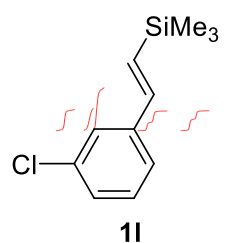

11

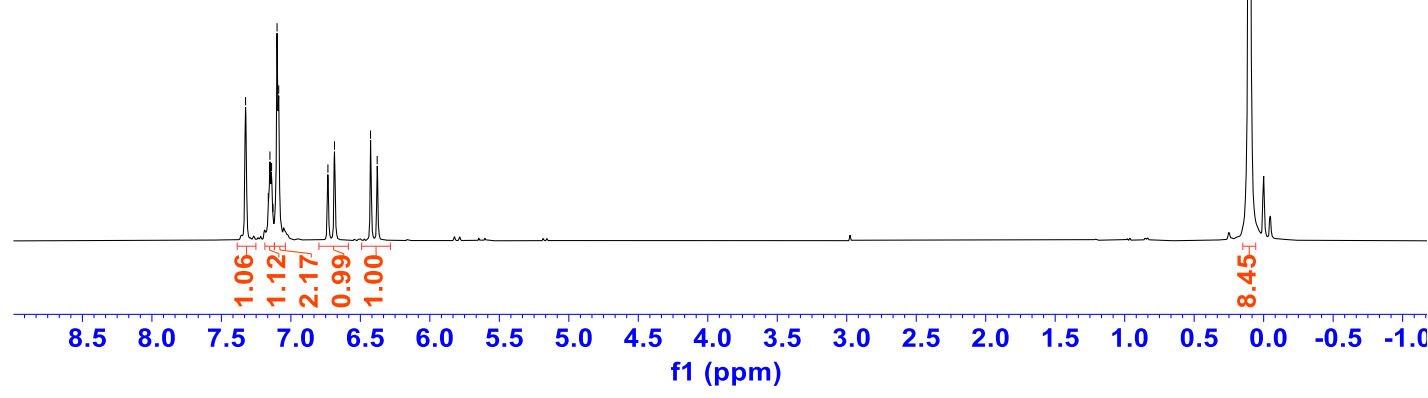

${ }^{13} \mathrm{C}\left\{{ }^{1} \mathrm{H}\right\}$ NMR of $11\left(100 \mathrm{MHZ}, \mathrm{CDCl}_{3}\right)$

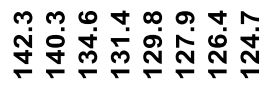

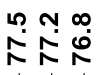

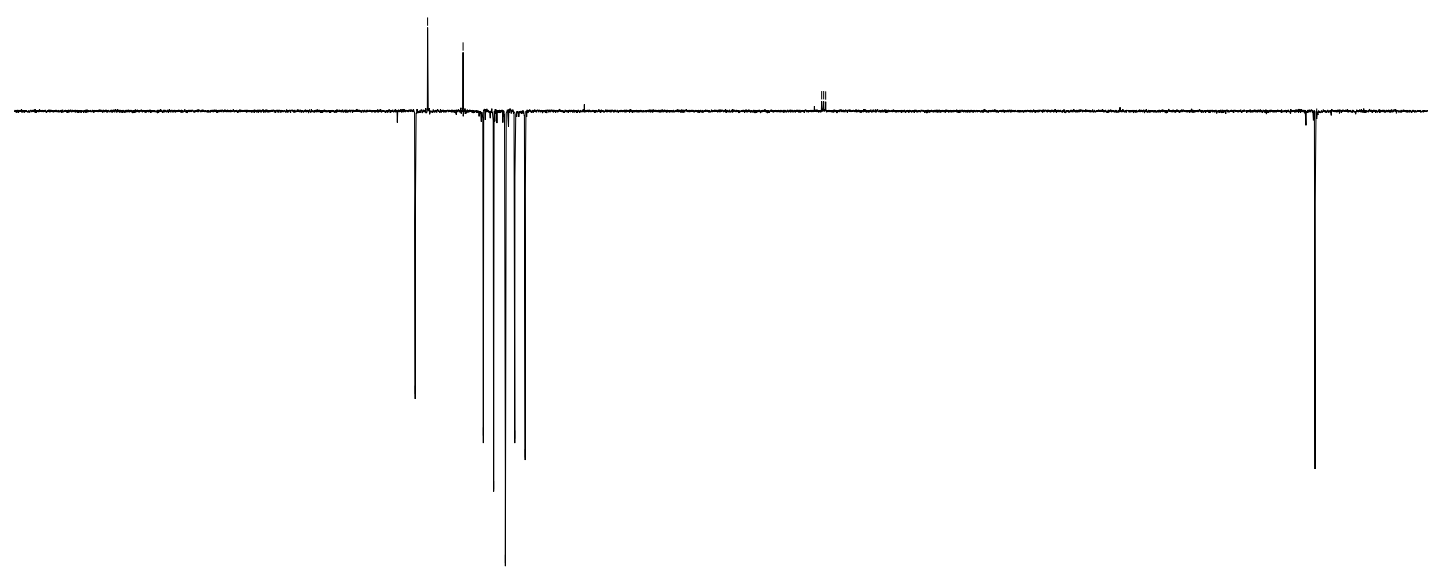

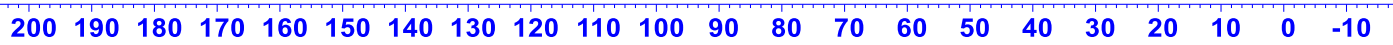
f1 (ppm) 
${ }^{1} \mathrm{H}$ NMR of $\mathbf{1 m}\left(400 \mathrm{MHZ}, \mathrm{CDCl}_{3}\right)$

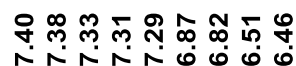

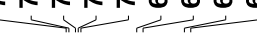
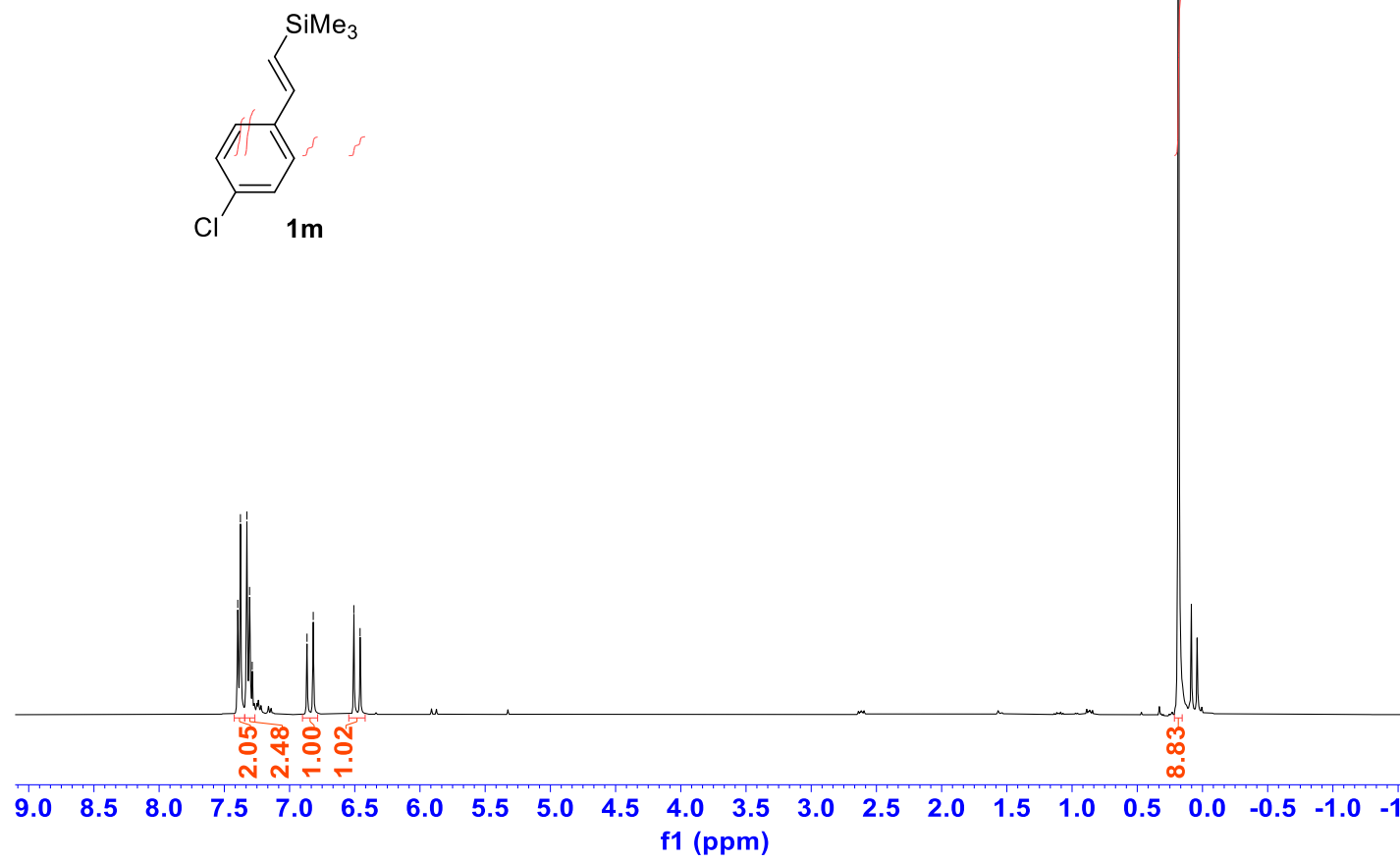

${ }^{13} \mathrm{C}\left\{{ }^{1} \mathrm{H}\right\}$ NMR of $\mathbf{1 m}\left(100 \mathrm{MHZ}, \mathrm{CDCl}_{3}\right)$

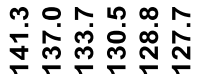

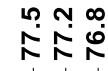

$\bar{i}$

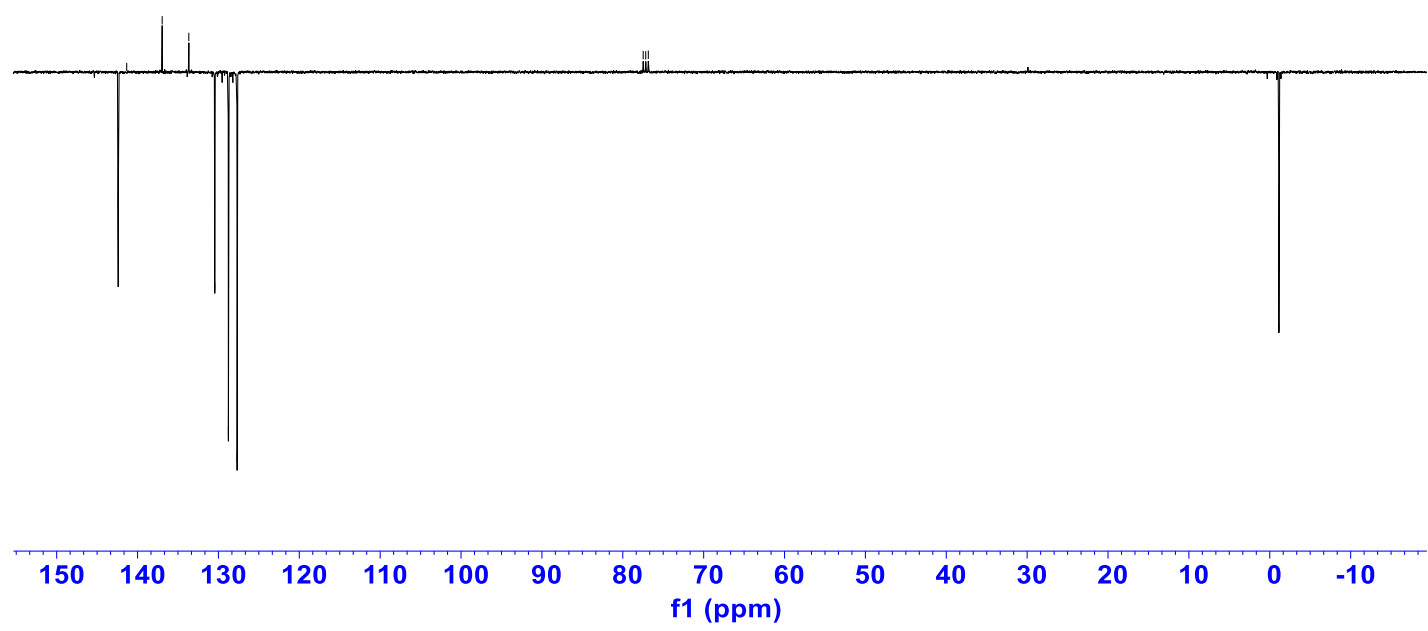


${ }^{1} \mathrm{H}$ NMR of 1 n (400 MHZ, $\left.\mathrm{CDCl}_{3}\right)$



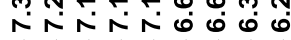
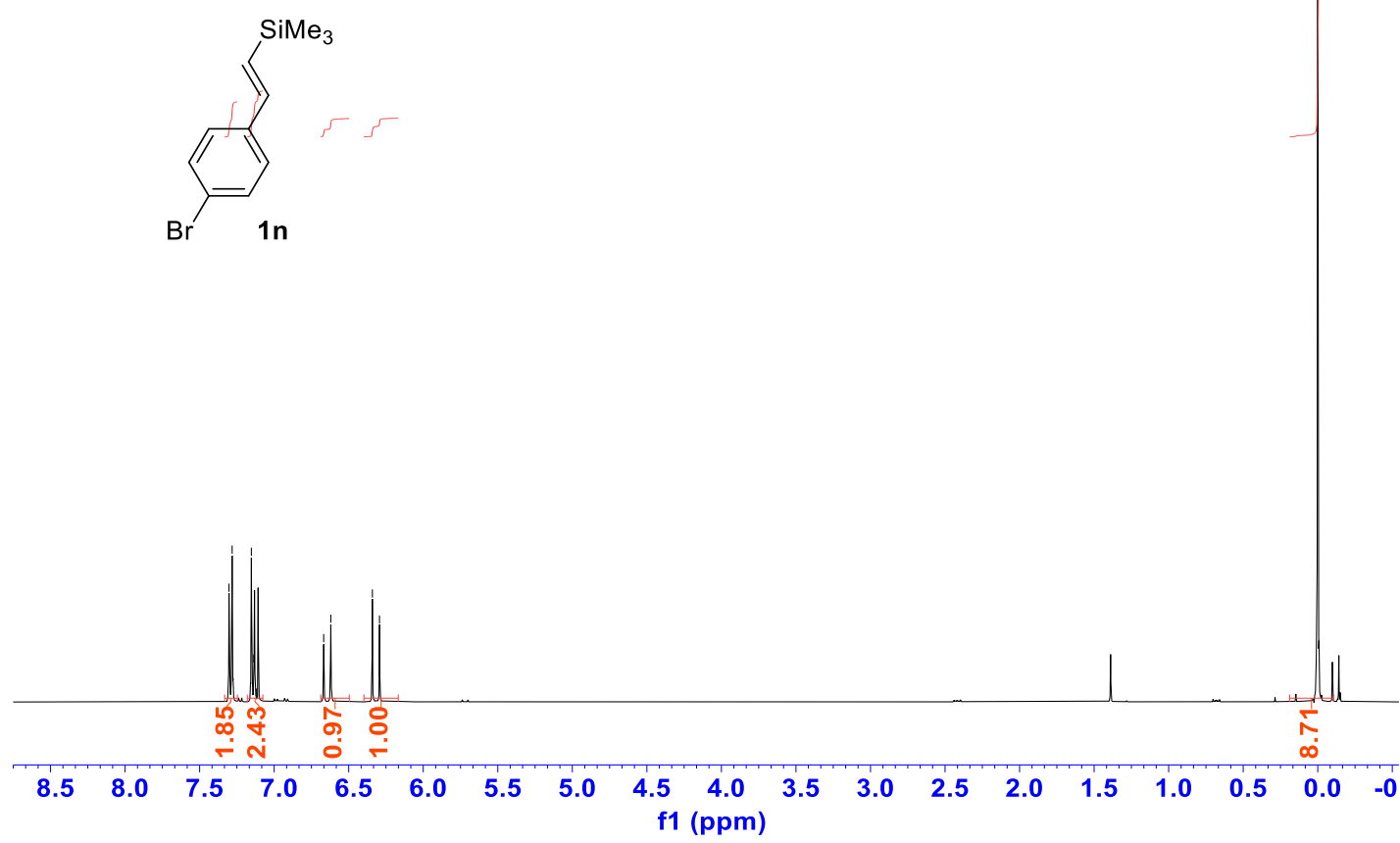

${ }^{13} \mathrm{C}\left\{{ }^{1} \mathrm{H}\right\}$ NMR of $\mathbf{1 n}\left(100 \mathrm{MHZ}, \mathrm{CDCl}_{3}\right)$

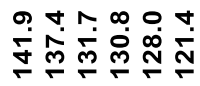

กิ กั

$\stackrel{\text { Nִ }}{i}$

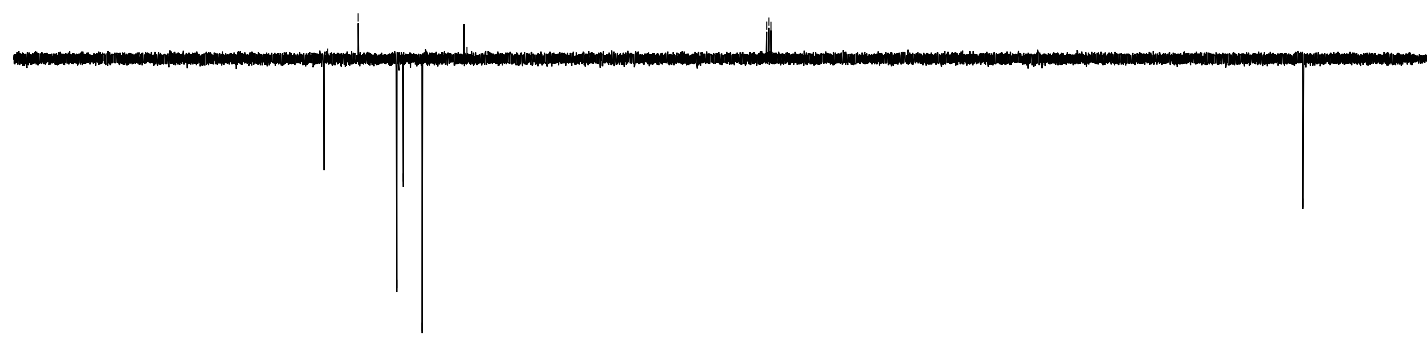

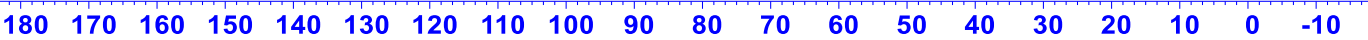
f1 (ppm) 
${ }^{1} \mathrm{H}$ NMR of $10\left(400 \mathrm{MHZ}, \mathrm{CDCl}_{3}\right)$

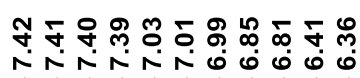

舟
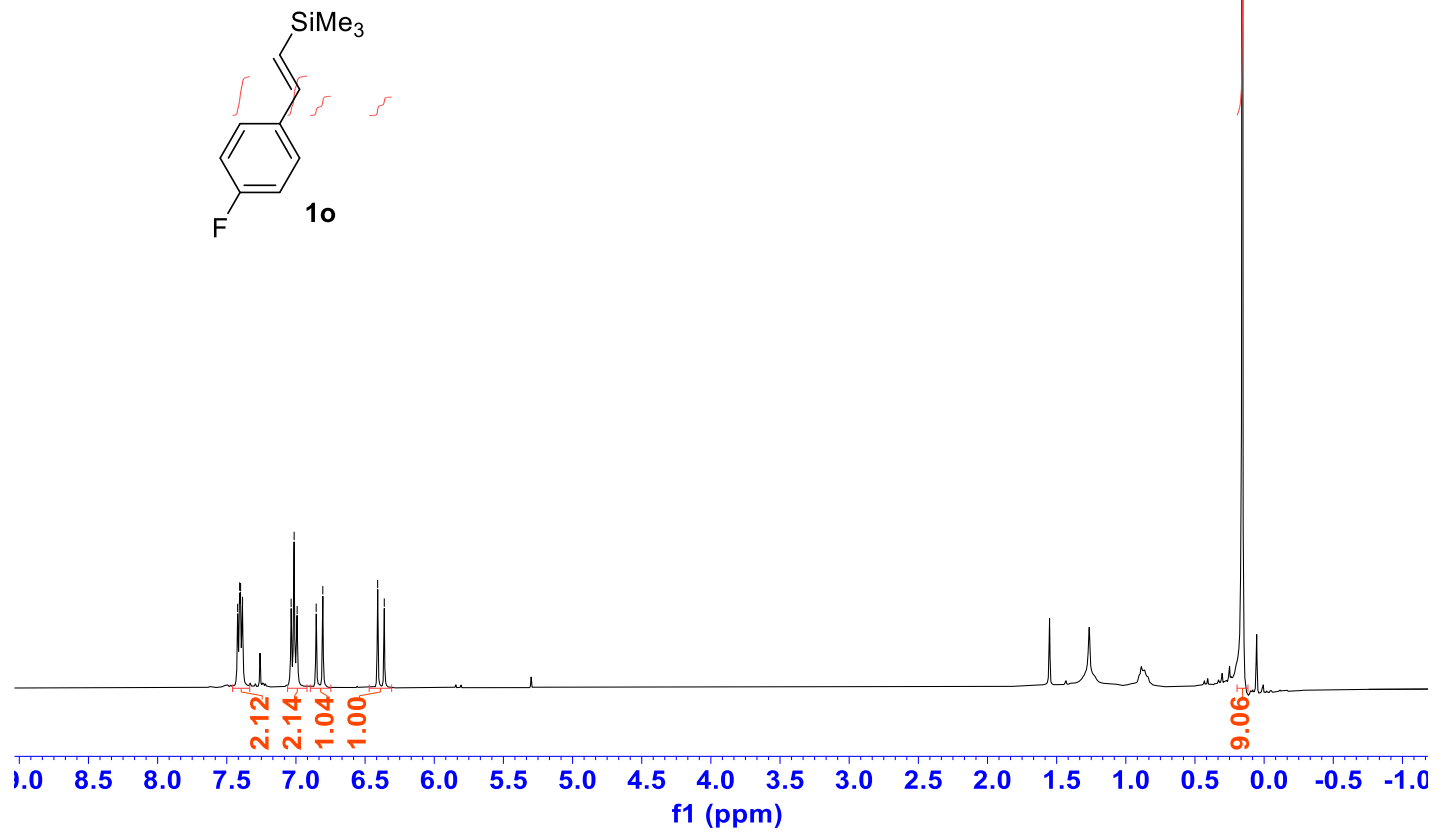

${ }^{13} \mathrm{C}\left\{{ }^{1} \mathrm{H}\right\}$ NMR of $10\left(100 \mathrm{MHZ}, \mathrm{CDCl}_{3}\right)$

운 눙

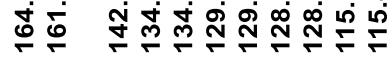

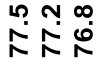

$\stackrel{\infty}{i}$

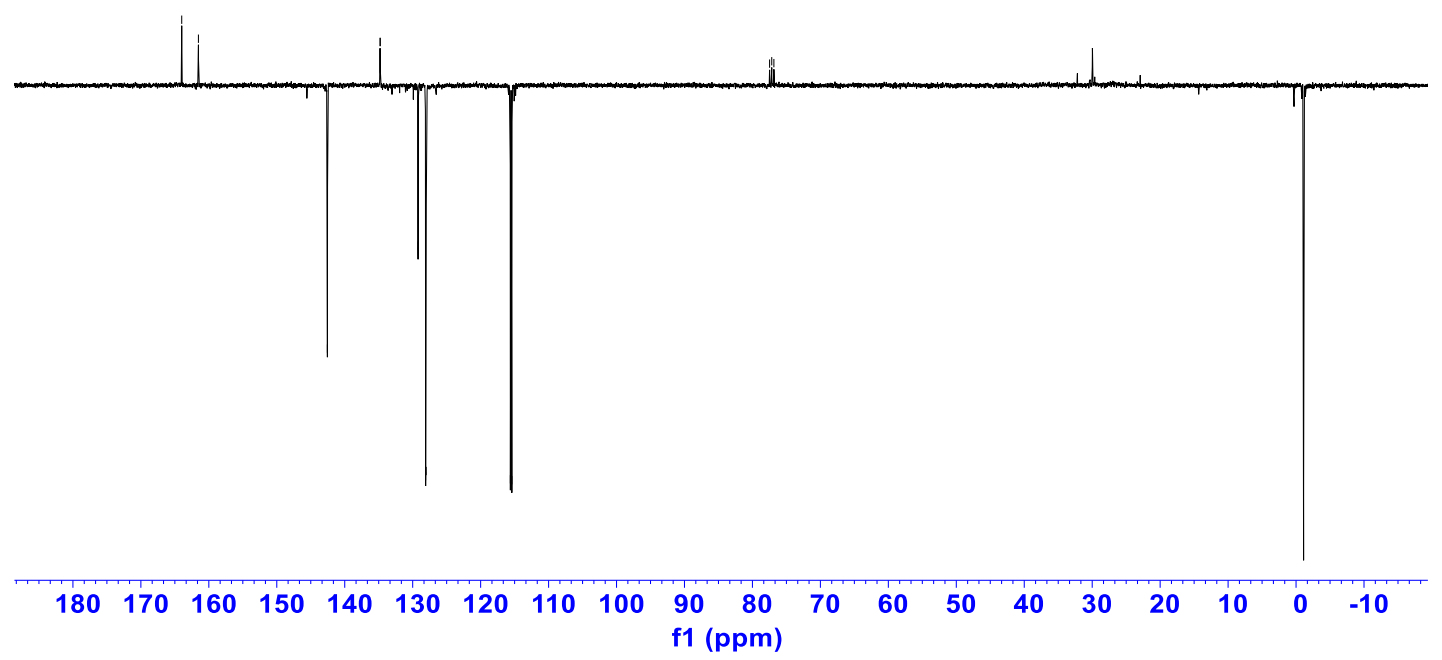


${ }^{1} \mathrm{H}$ NMR of 1p (400 MHZ, $\mathrm{CDCl}_{3}$ )

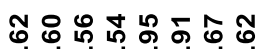

NNN年

(sime

( 
${ }^{1} \mathrm{H} \mathrm{NMR}$ of $\mathbf{1 q}\left(400 \mathrm{MHZ}, \mathrm{CDCl}_{3}\right)$

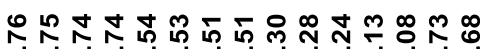

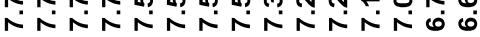
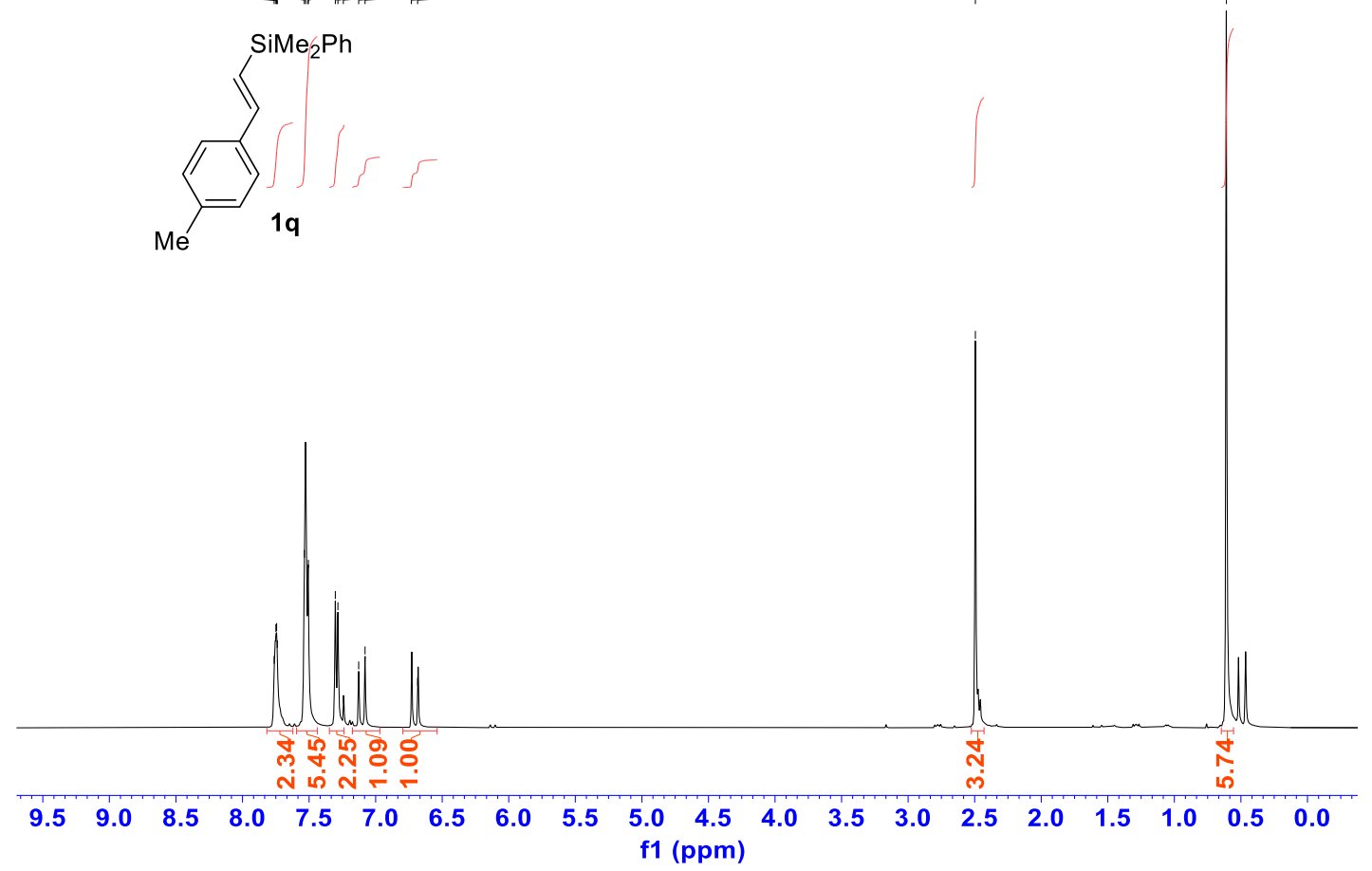

${ }^{13} \mathrm{C}\left\{{ }^{1} \mathrm{H}\right\}$ NMR of 1q $\left(100 \mathrm{MHZ}, \mathrm{CDCl}_{3}\right)$

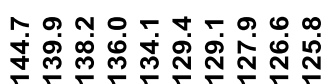

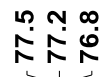

$\stackrel{\grave{n}}{\dot{4}}$

$\stackrel{m}{i}$

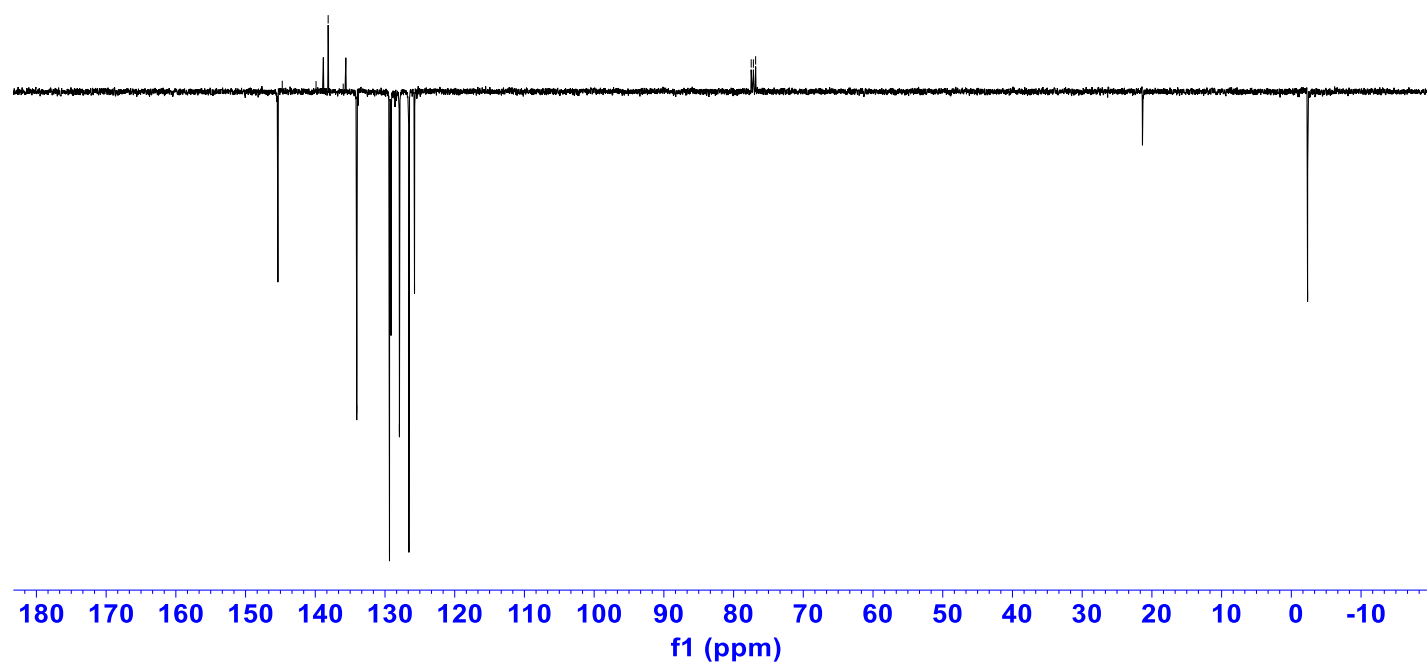


${ }^{1} \mathrm{H} \mathrm{NMR}$ of $1 \mathbf{r}\left(400 \mathrm{MHZ}, \mathrm{CDCl}_{3}\right)$

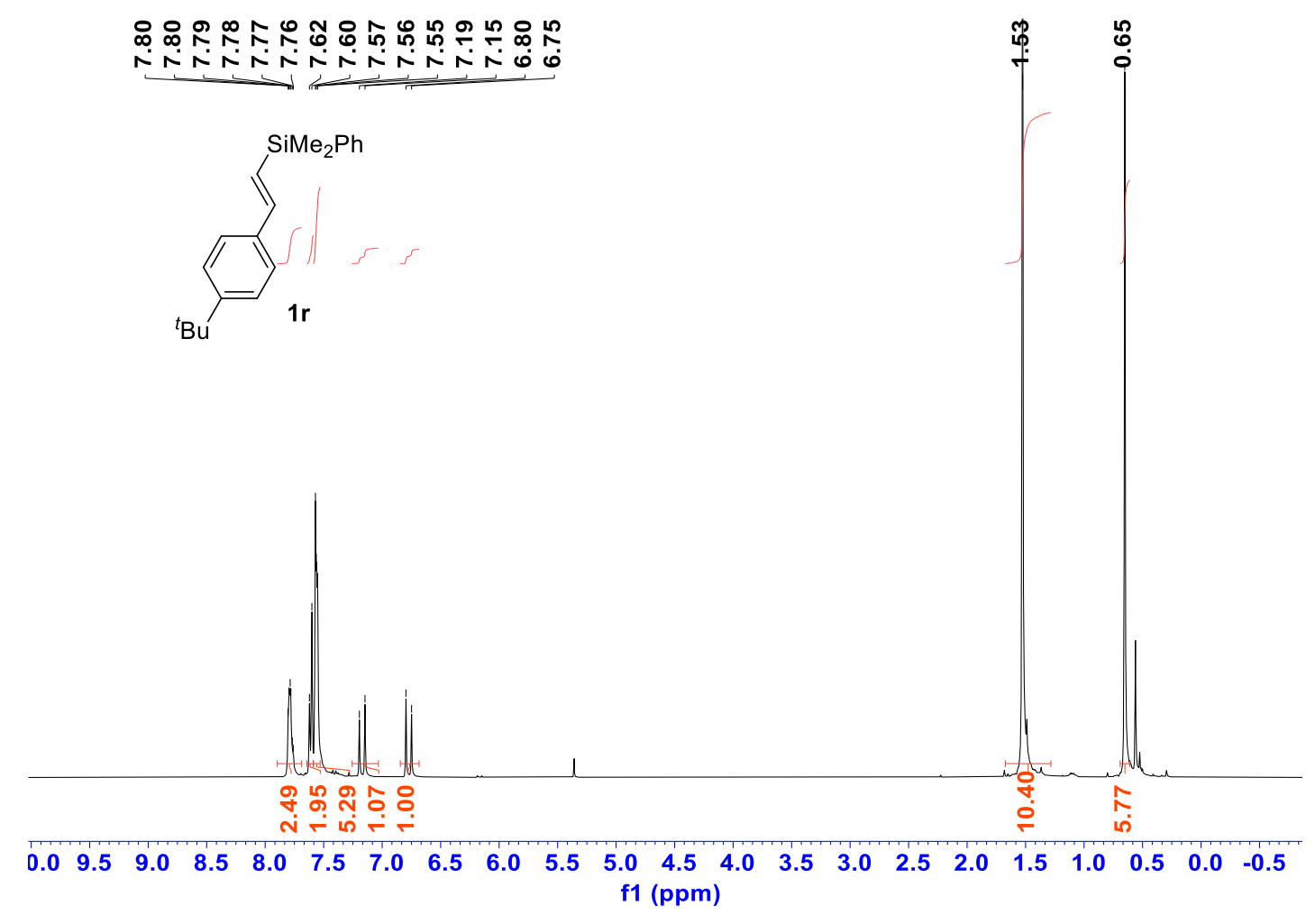

${ }^{13} \mathrm{C}\left\{{ }^{1} \mathrm{H}\right\}$ NMR of 1r $\left(100 \mathrm{MHZ}, \mathrm{CDCl}_{3}\right)$

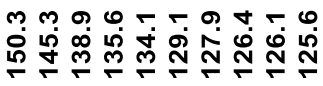

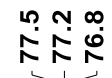

过

$\ddot{p}$

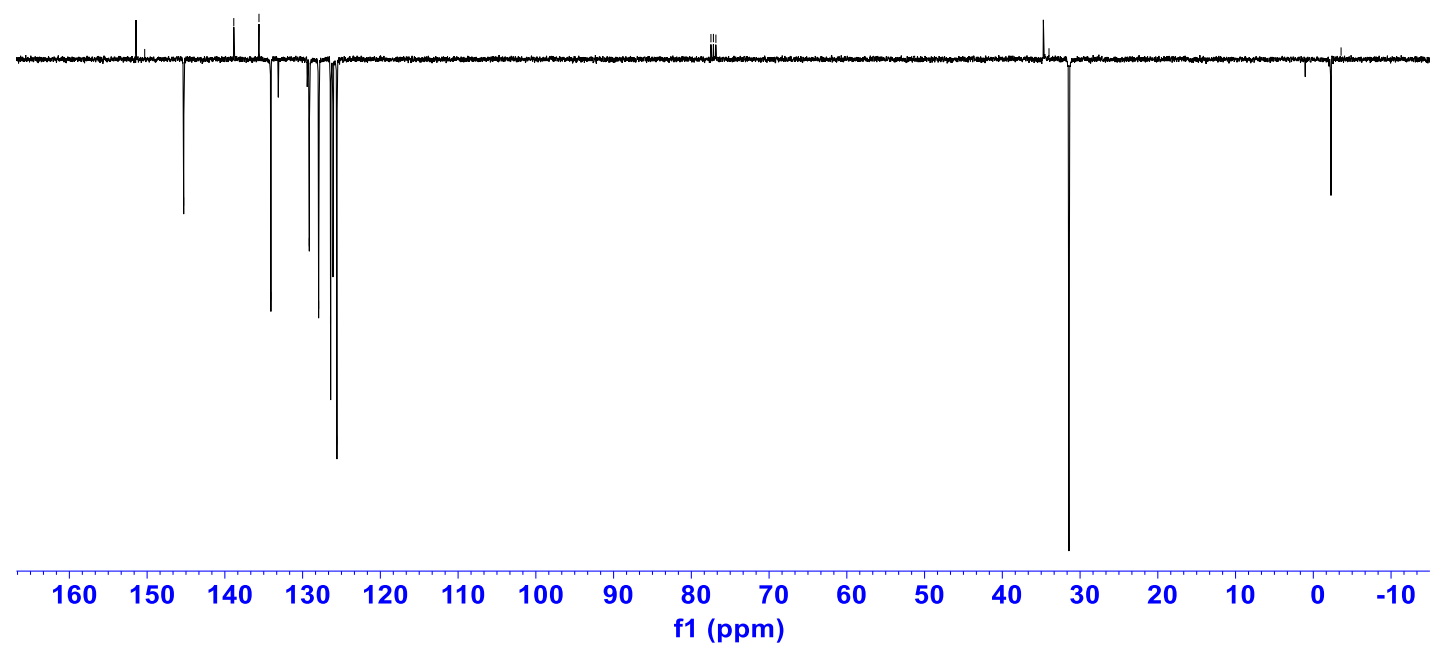


${ }^{1} \mathrm{H} \mathrm{NMR}$ of $1 \mathrm{~s}\left(400 \mathrm{MHZ}, \mathrm{CDCl}_{3}\right)$

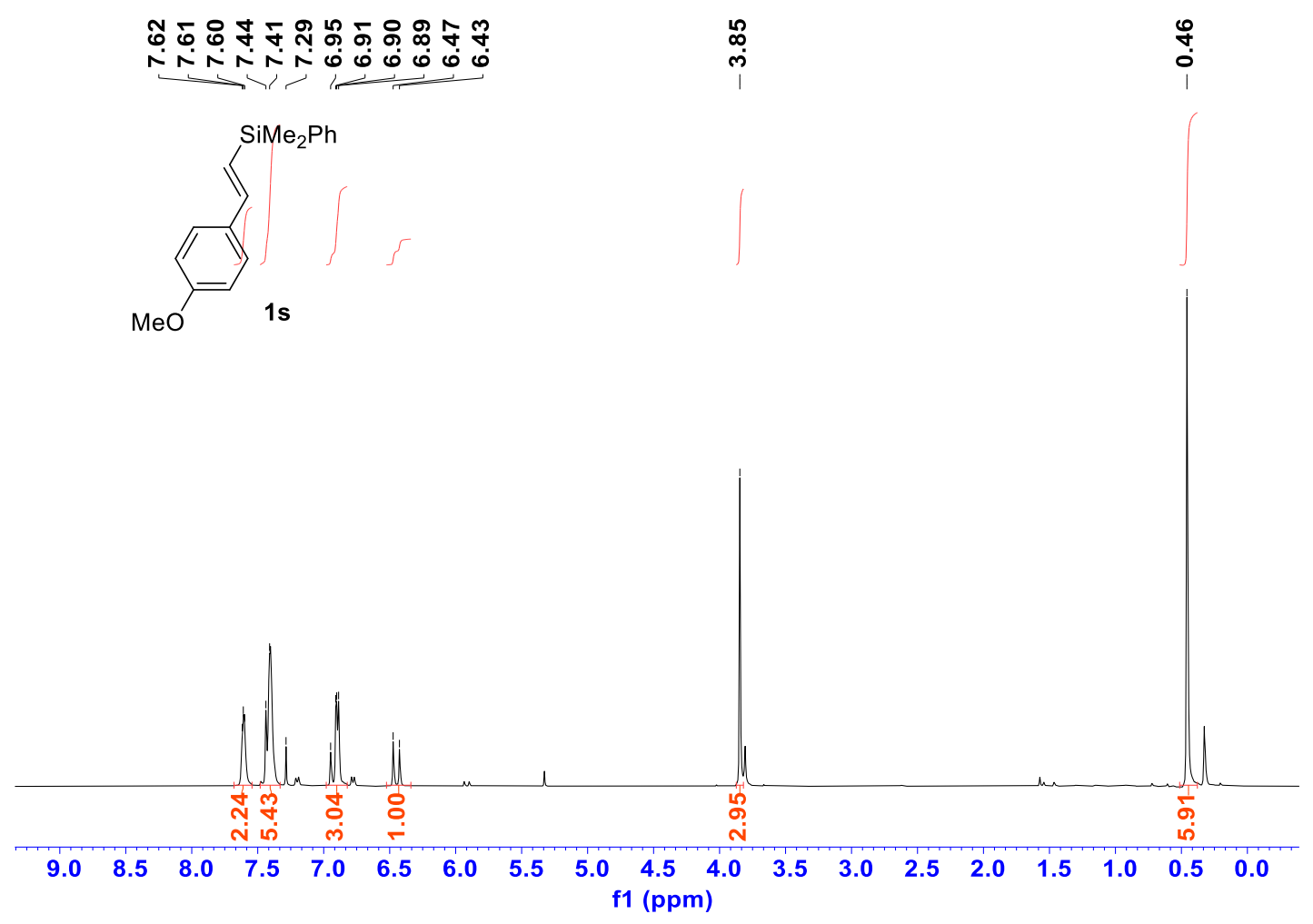

${ }^{13} \mathrm{C}\left\{{ }^{1} \mathrm{H}\right\}$ NMR of $\mathbf{1 s}\left(100 \mathrm{MHZ}, \mathrm{CDCl}_{3}\right)$
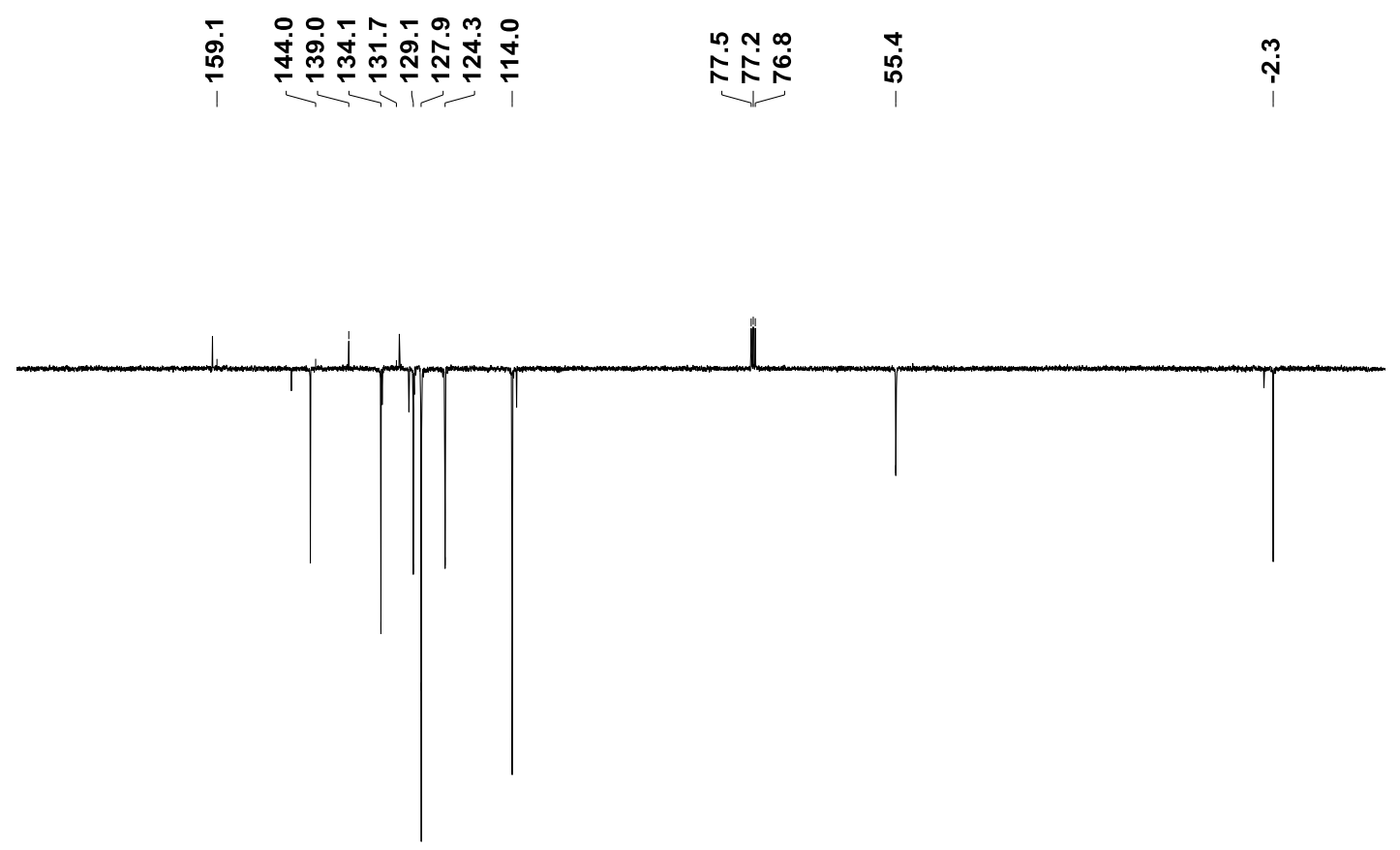

$\begin{array}{lllllllllllllllllllll}180 & 170 & 160 & 150 & 140 & 130 & 120 & 110 & 100 & 90 & 80 & 70 & 60 & 50 & 40 & 30 & 20 & 10 & 0 & -10\end{array}$ f1 (ppm) 


\section{${ }^{1} \mathrm{H}$ NMR of 1t (400 MHZ, $\left.\mathrm{CDCl}_{3}\right)$}

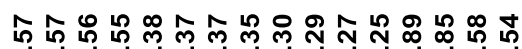

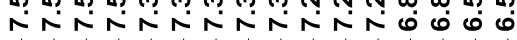

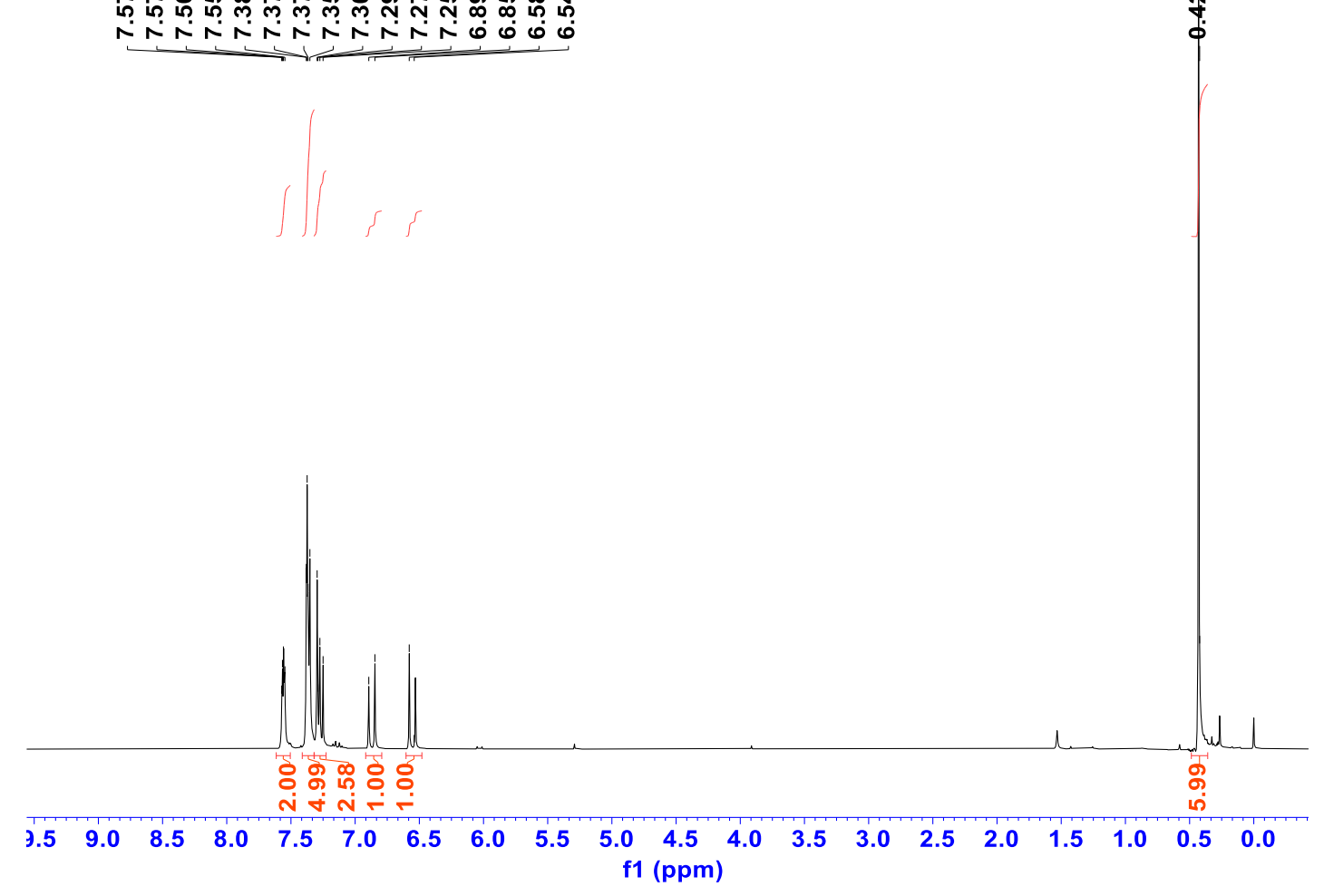

${ }^{13} \mathrm{C}\left\{{ }^{1} \mathrm{H}\right\}$ NMR of $\mathbf{1 t}\left(100 \mathrm{MHZ}, \mathrm{CDCl}_{3}\right)$

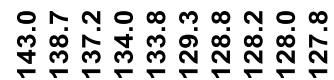

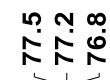

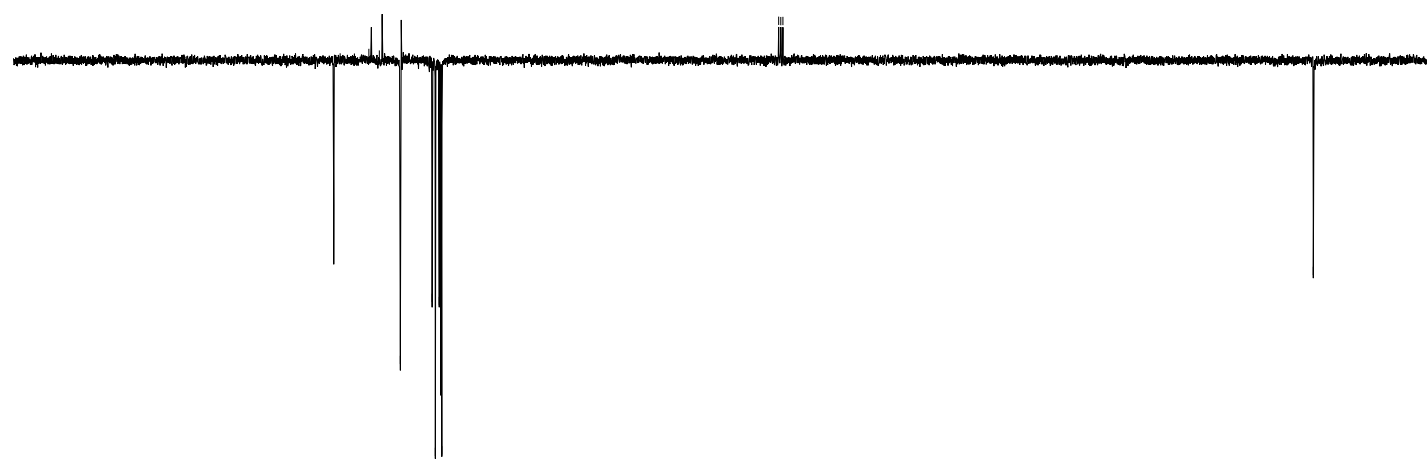

$\begin{array}{llllllllllllllllllllll}190 & 180 & 170 & 160 & 150 & 140 & 130 & 120 & 110 & 100 & \begin{array}{rl}90 & 80 \\ \mathrm{f} 1(\mathrm{ppm})\end{array} & 70 & 60 & 50 & 40 & 30 & 20 & 10 & 0 & -10\end{array}$ 
${ }^{1} \mathrm{H} \mathrm{NMR}$ of $\mathbf{1 u}\left(400 \mathrm{MHZ}, \mathrm{CDCl}_{3}\right)$

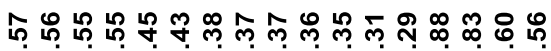

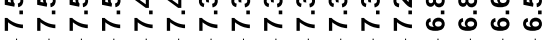
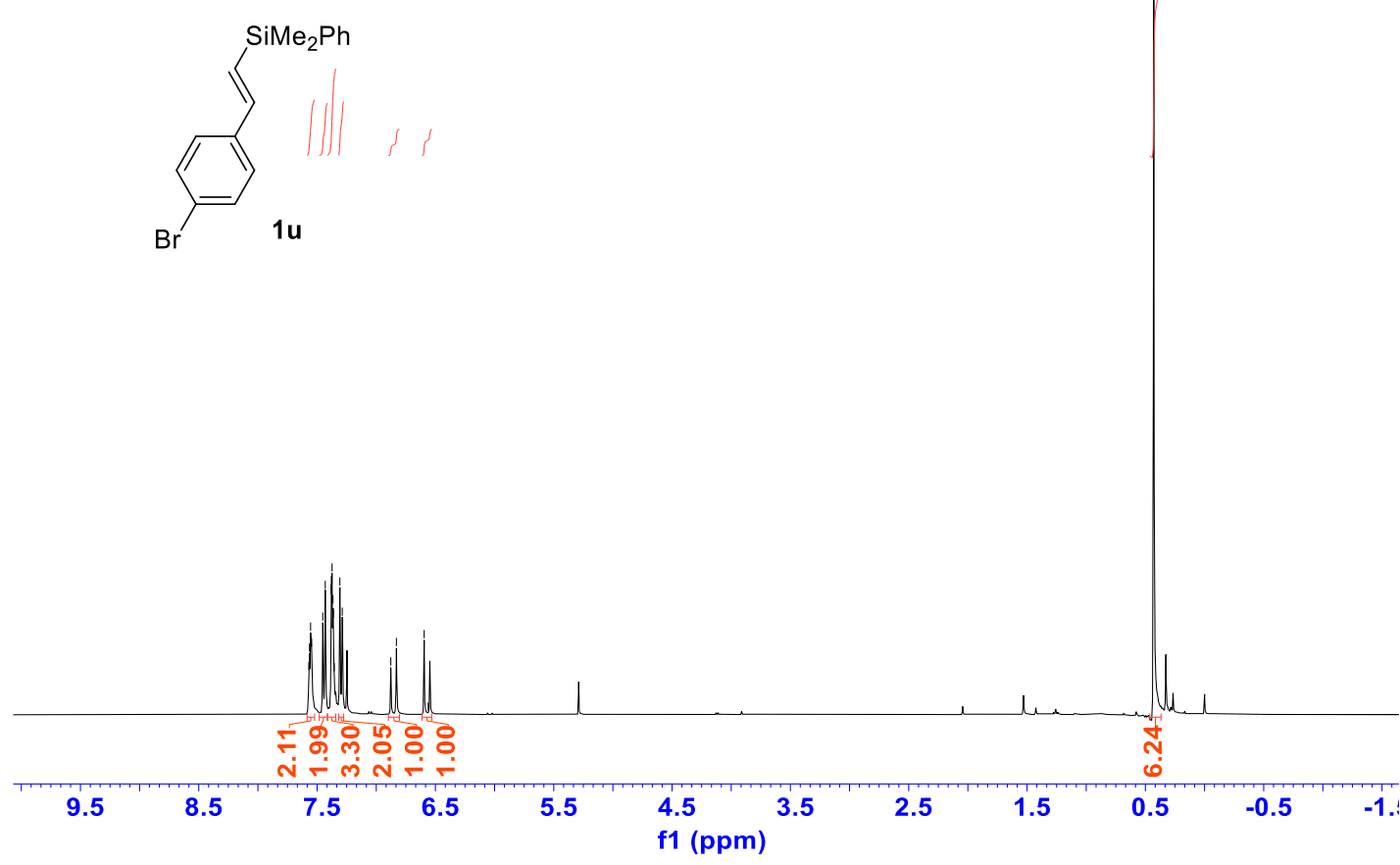

${ }^{13} \mathrm{C}\left\{{ }^{1} \mathrm{H}\right\}$ NMR of $\mathbf{1 u}\left(100 \mathrm{MHZ}, \mathrm{CDCl}_{3}\right)$

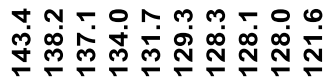

مก

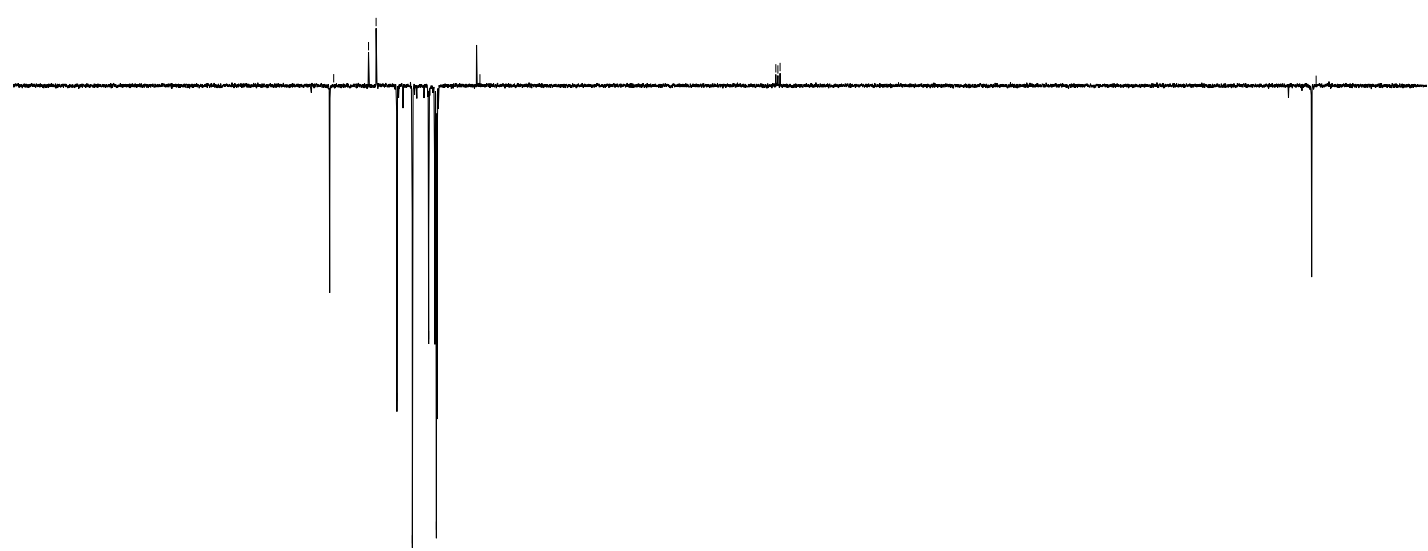

$\begin{array}{lllllllllllllllllllll}90 & 180 & 170 & 160 & 150 & 140 & 130 & 120 & 110 & 100 & 90 & 80 & 70 & 60 & 50 & 40 & 30 & 20 & 10 & 0 & -10\end{array}$ f1 (ppm) 
${ }^{1} \mathrm{H}$ NMR of $1 \mathbf{v}\left(400 \mathrm{MHZ}, \mathrm{CDCl}_{3}\right)$

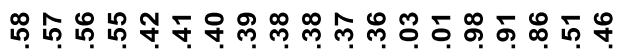

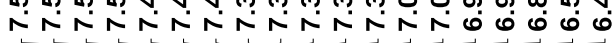
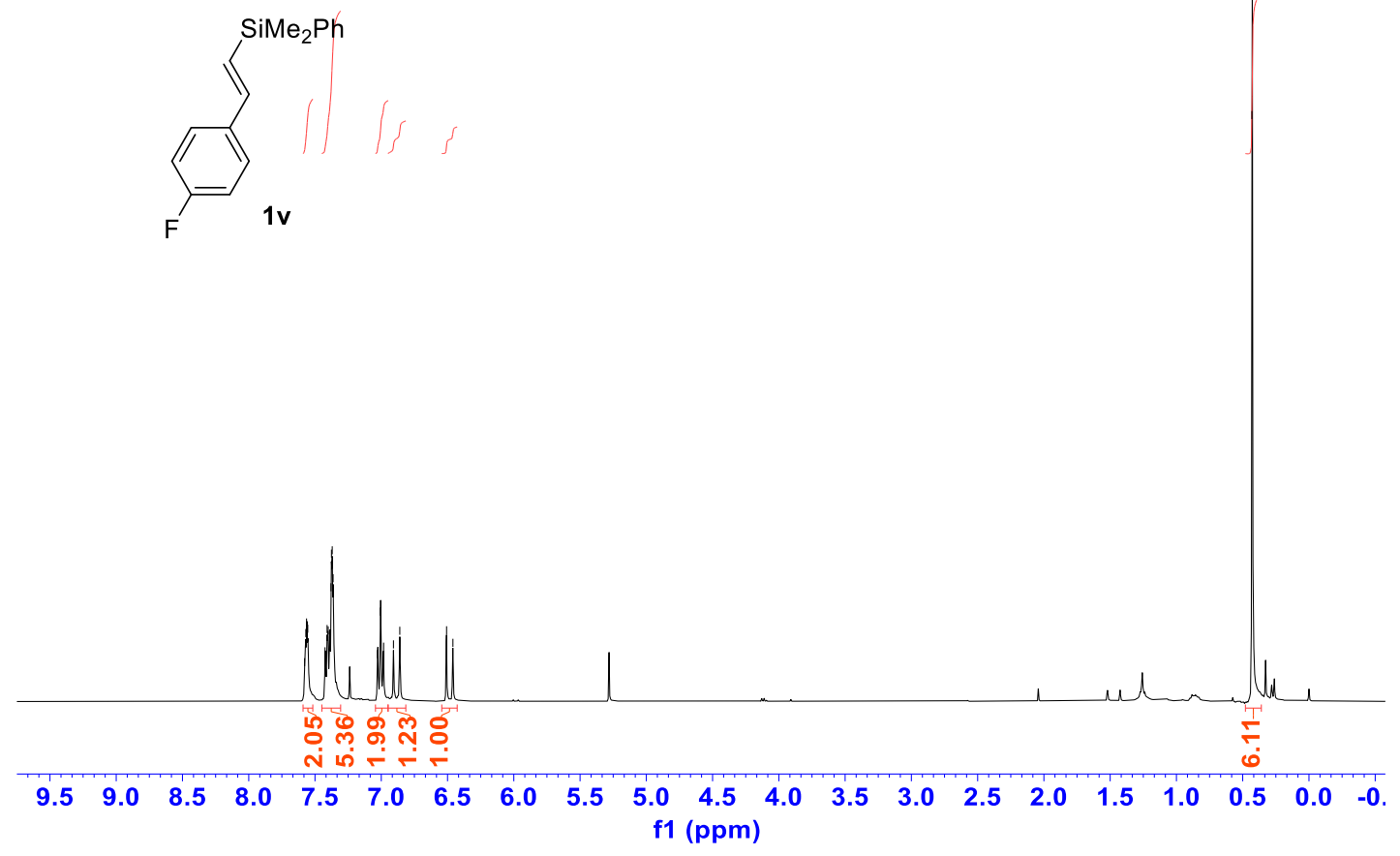

${ }^{13} \mathrm{C}\left\{{ }^{1} \mathrm{H}\right\}$ NMR of $\mathbf{1 v}\left(100 \mathrm{MHZ}, \mathrm{CDCl}_{3}\right)$

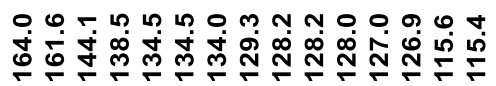

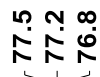

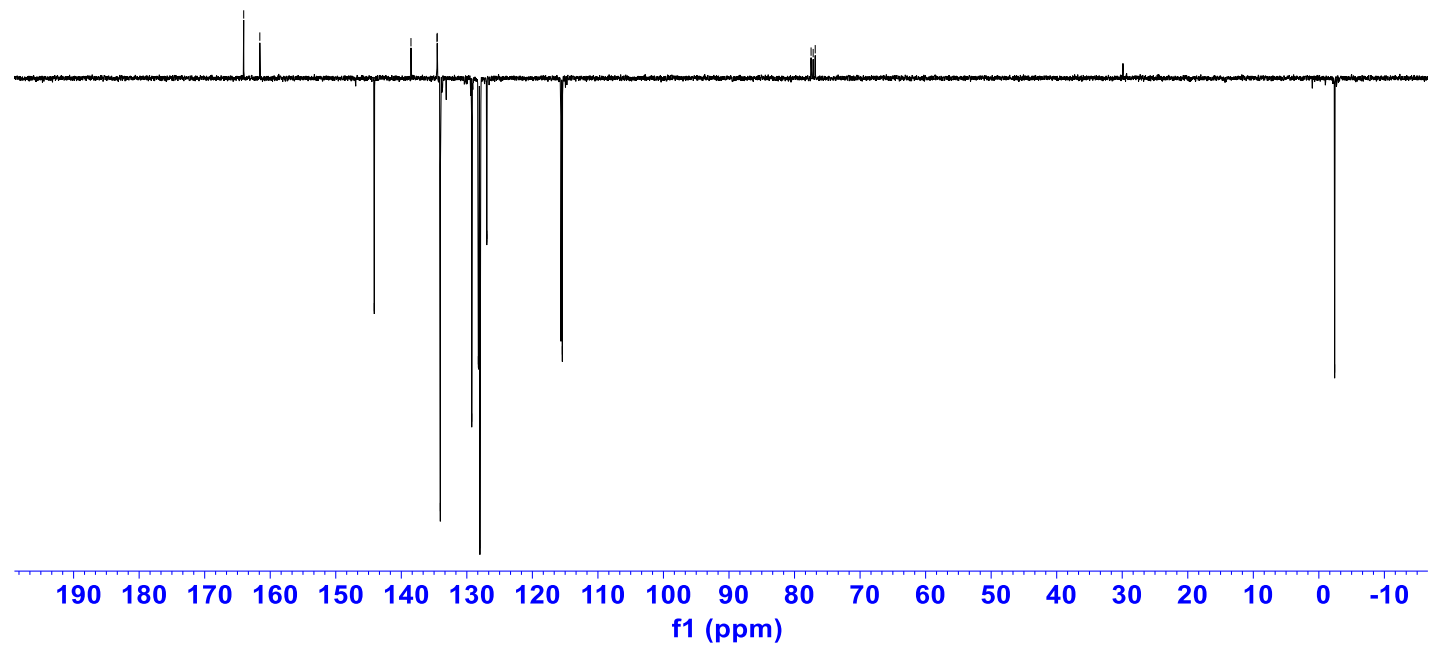


${ }^{1} \mathrm{H} \mathrm{NMR}$ of $\mathbf{1 w}\left(400 \mathrm{MHZ}, \mathrm{CDCl}_{3}\right)$

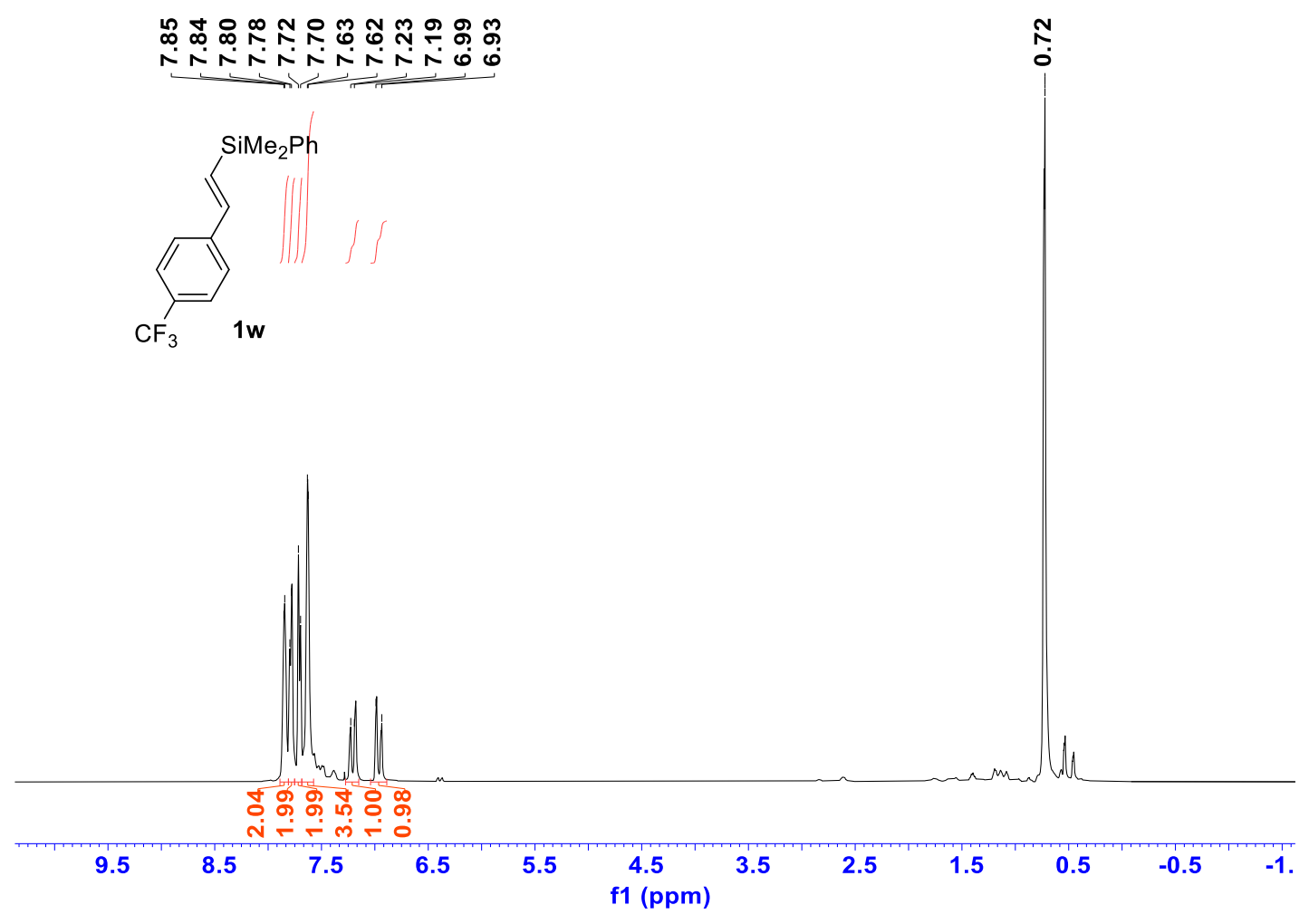

${ }^{13} \mathrm{C}\left\{{ }^{1} \mathrm{H}\right\}$ NMR of $\mathbf{1 w}\left(100 \mathrm{MHZ}, \mathrm{CDCl}_{3}\right)$

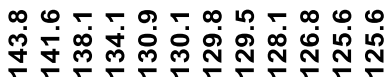

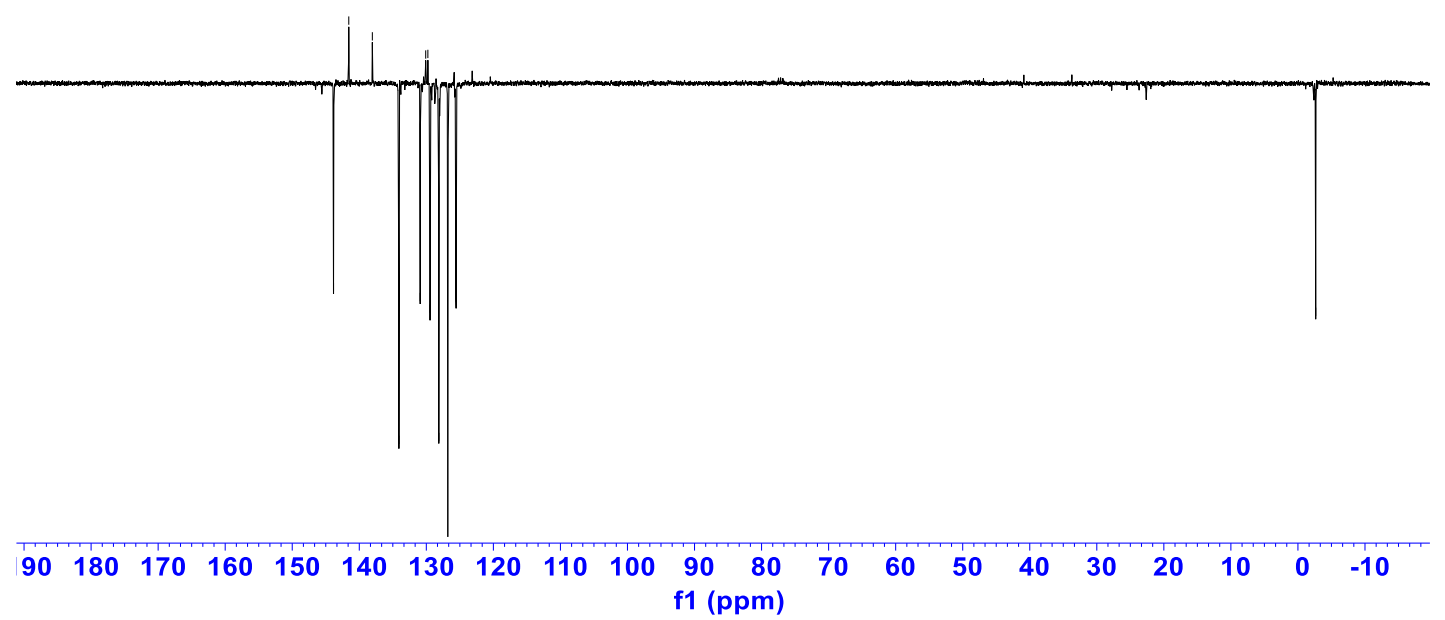


${ }^{1} \mathrm{H}$ NMR of 3a (400 MHZ, $\left.\mathrm{CDCl}_{3}\right)$

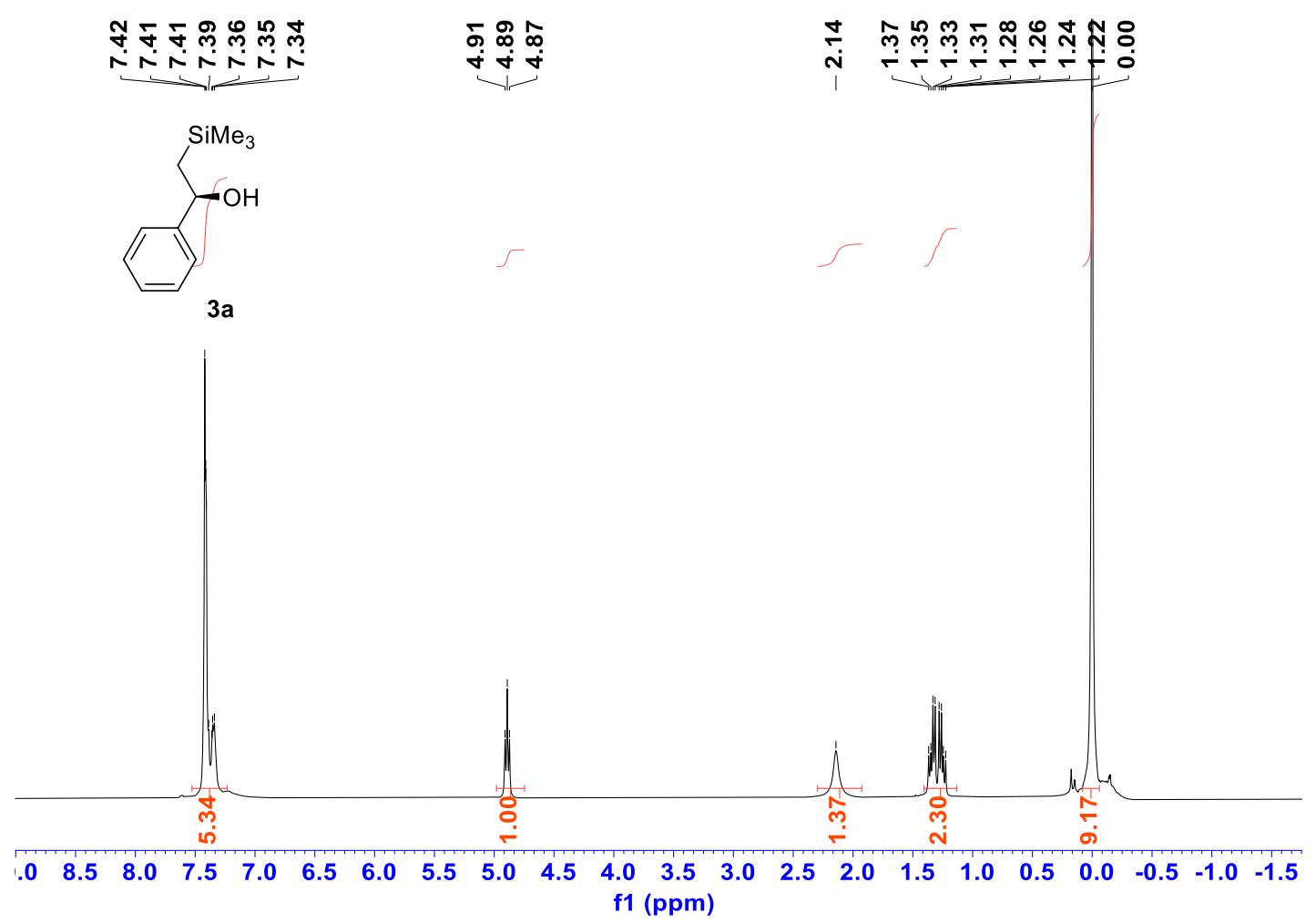

${ }^{13} \mathrm{C}\left\{{ }^{1} \mathrm{H}\right\}$ NMR of 3a $\left(100 \mathrm{MHZ}, \mathrm{CDCl}_{3}\right)$

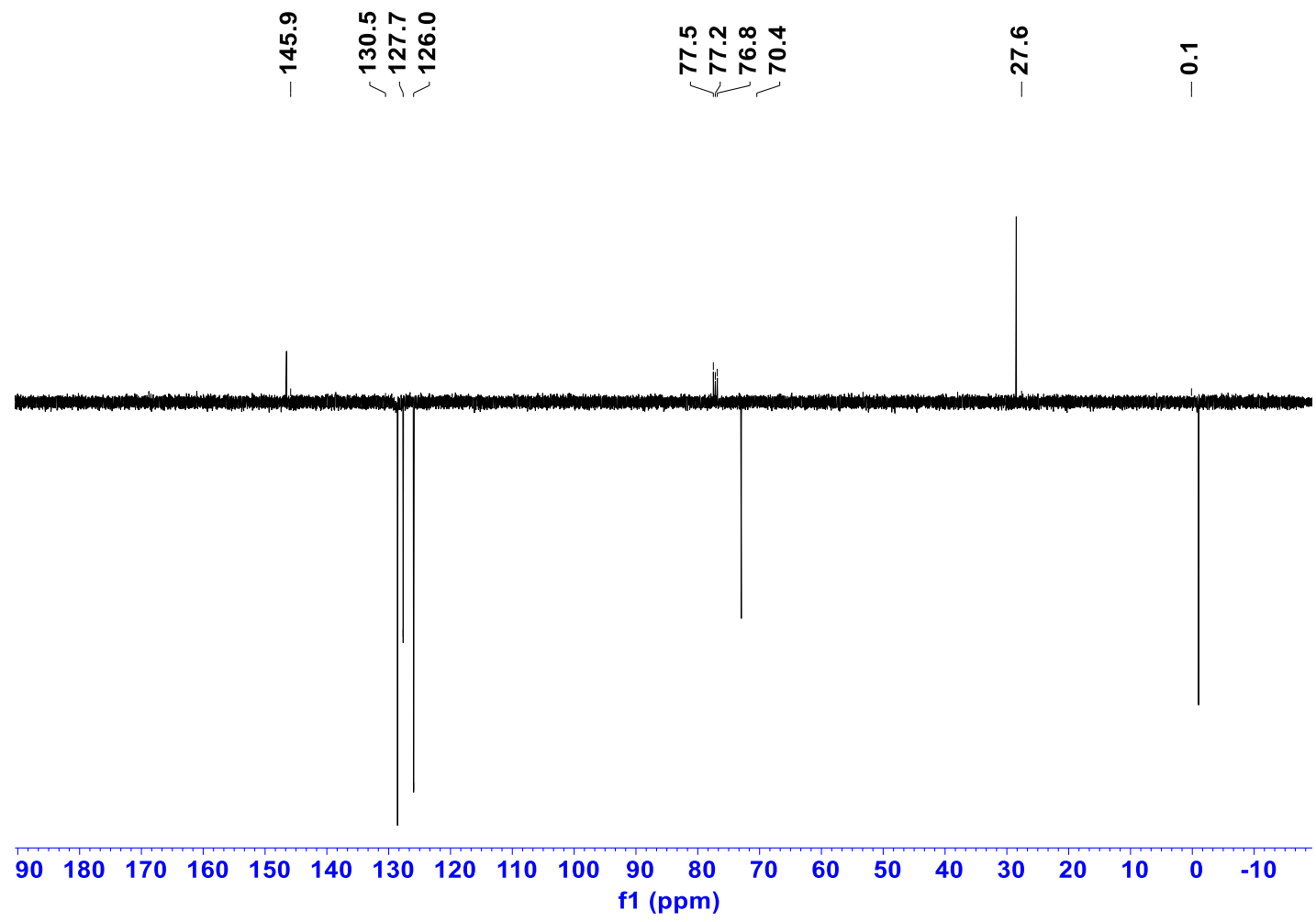


${ }^{1} \mathrm{H}$ NMR of $5 \mathbf{c}\left(400 \mathrm{MHZ}, \mathrm{CDCl}_{3}\right)$

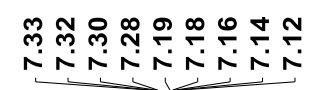<smiles>C[As](CO)C(C)(C)c1ccccc1</smiles>

$5 c$

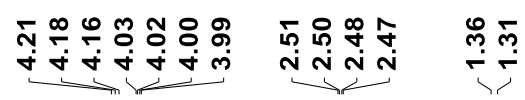

\section{列}

$\$$
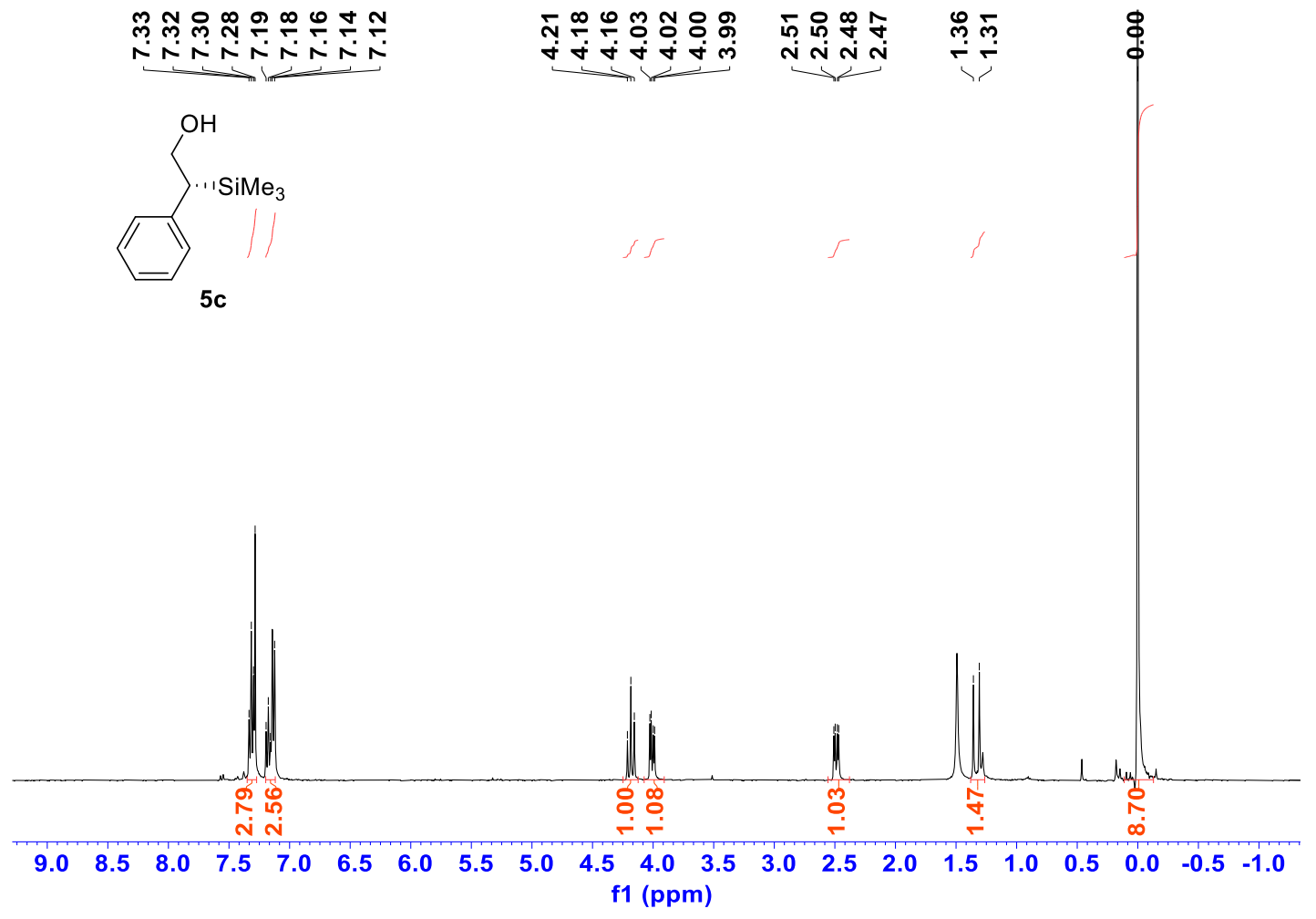

${ }^{13} \mathrm{C}\left\{{ }^{1} \mathrm{H}\right\}$ NMR of $5 \mathbf{c}\left(100 \mathrm{MHZ}, \mathrm{CDCl}_{3}\right)$

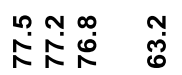

in

$\stackrel{\text { กิ }}{1}$

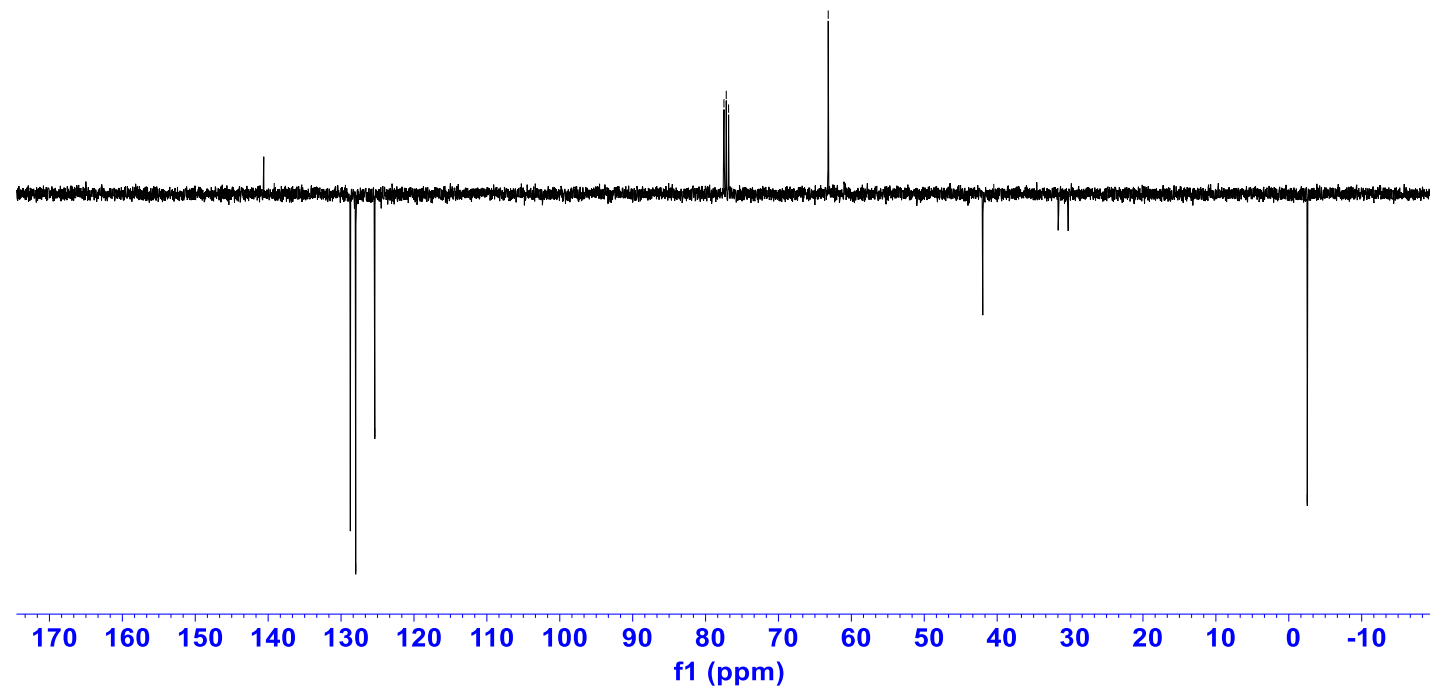


${ }^{1} \mathrm{H}$ NMR of $\mathbf{3 b}\left(400 \mathrm{MHZ}, \mathrm{CDCl}_{3}\right)$

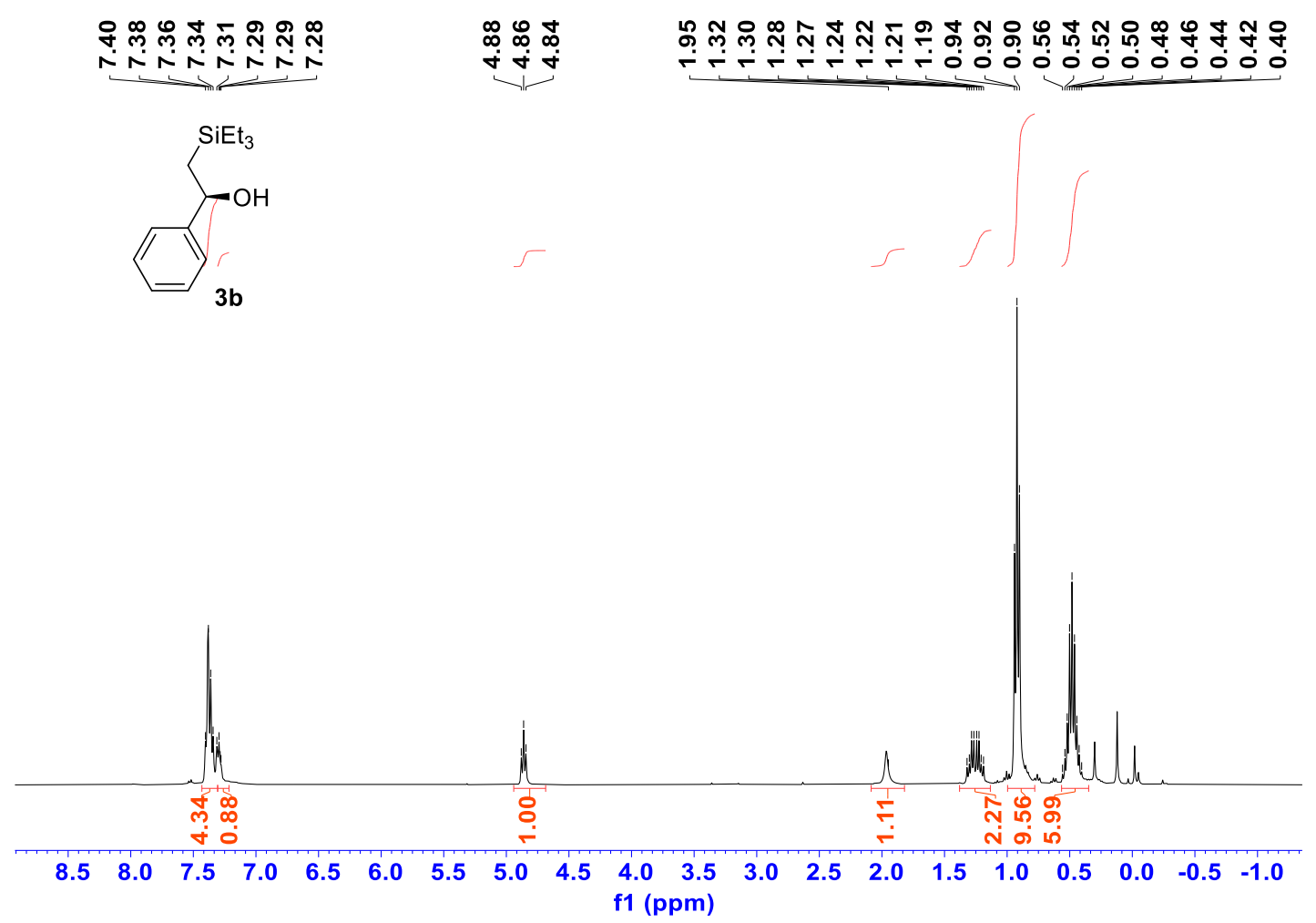

${ }^{13} \mathrm{C}\left\{{ }^{1} \mathrm{H}\right\}$ NMR of $\mathbf{3 b}\left(100 \mathrm{MHZ}, \mathrm{CDCl}_{3}\right)$

\begin{tabular}{|c|c|c|c|}
\hline 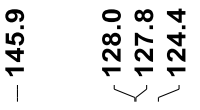 & $\underbrace{n N o}$ & $\ddot{m}^{n}$ & 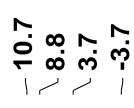 \\
\hline
\end{tabular}

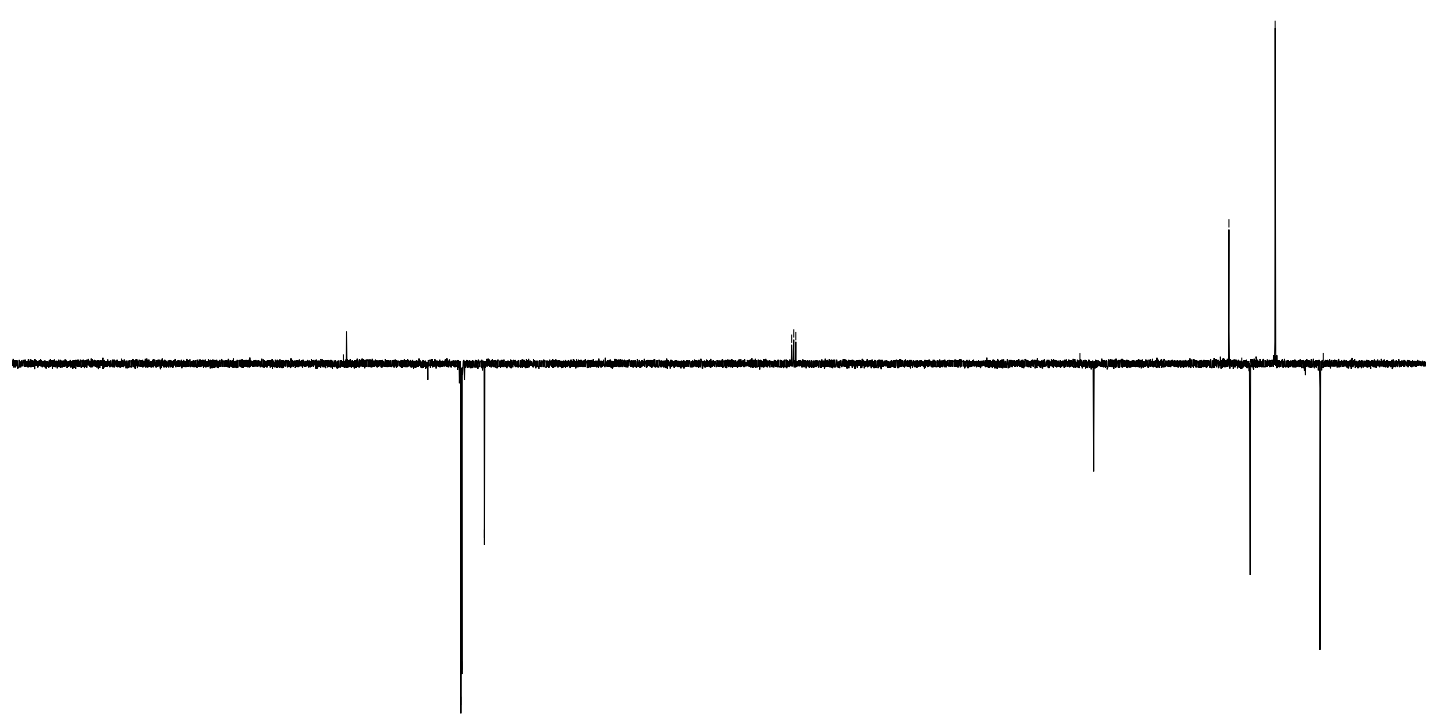

$\begin{array}{lllllllllllllllllllll}190 & 180 & 170 & 160 & 150 & 140 & 130 & 120 & 110 & 100 & 90 & 80 & 70 & 60 & 50 & 40 & 30 & 20 & 10 & 0 & -10\end{array}$ f1 (ppm) 
${ }^{1} \mathrm{H}$ NMR of $3 \mathbf{c}\left(400 \mathrm{MHZ}, \mathrm{CDCl}_{3}\right)$

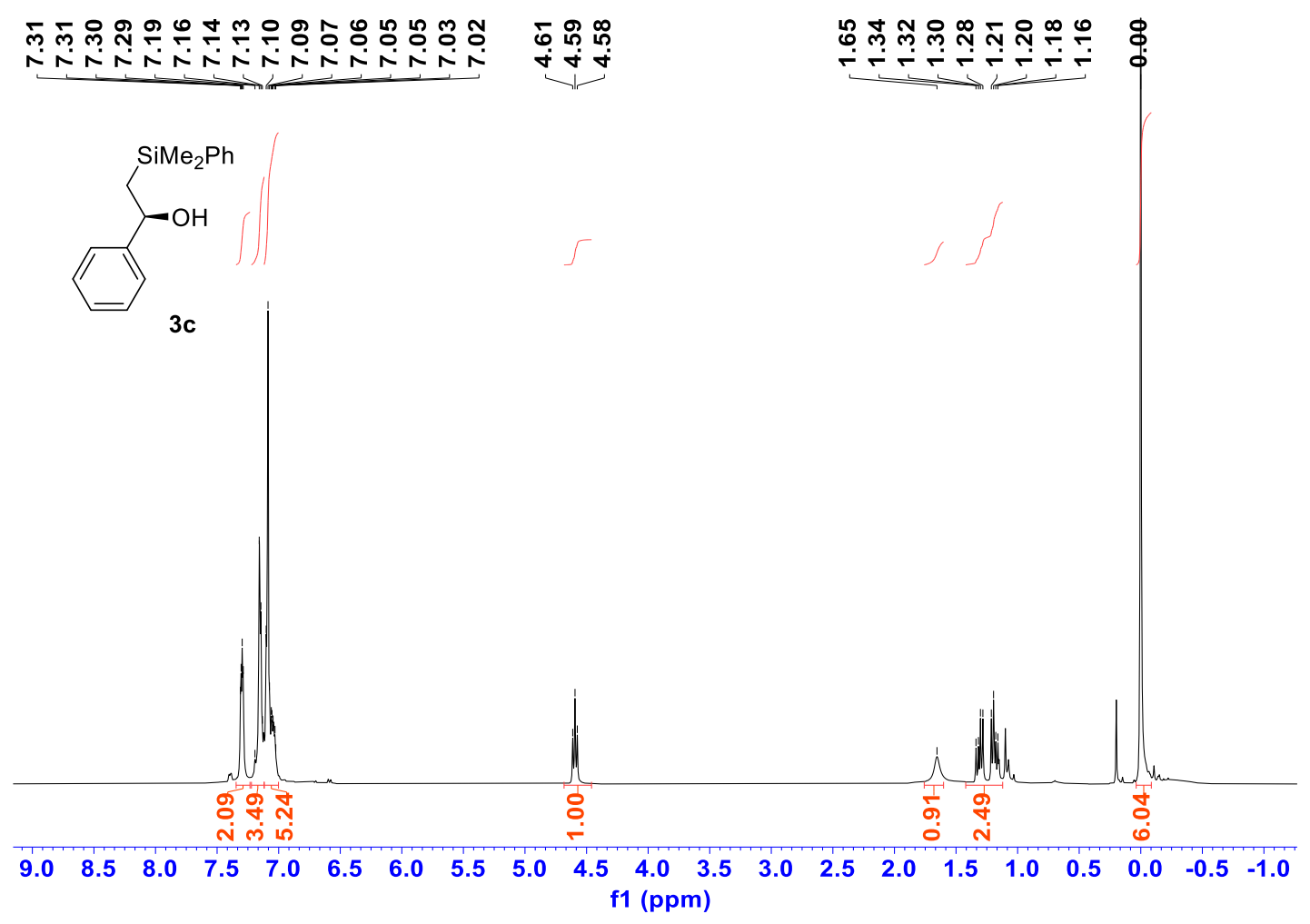

${ }^{13} \mathrm{C}\left\{{ }^{1} \mathrm{H}\right\}$ NMR of $\mathbf{3 c}\left(100 \mathrm{MHZ}, \mathrm{CDCl}_{3}\right)$

ำกำ

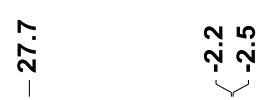

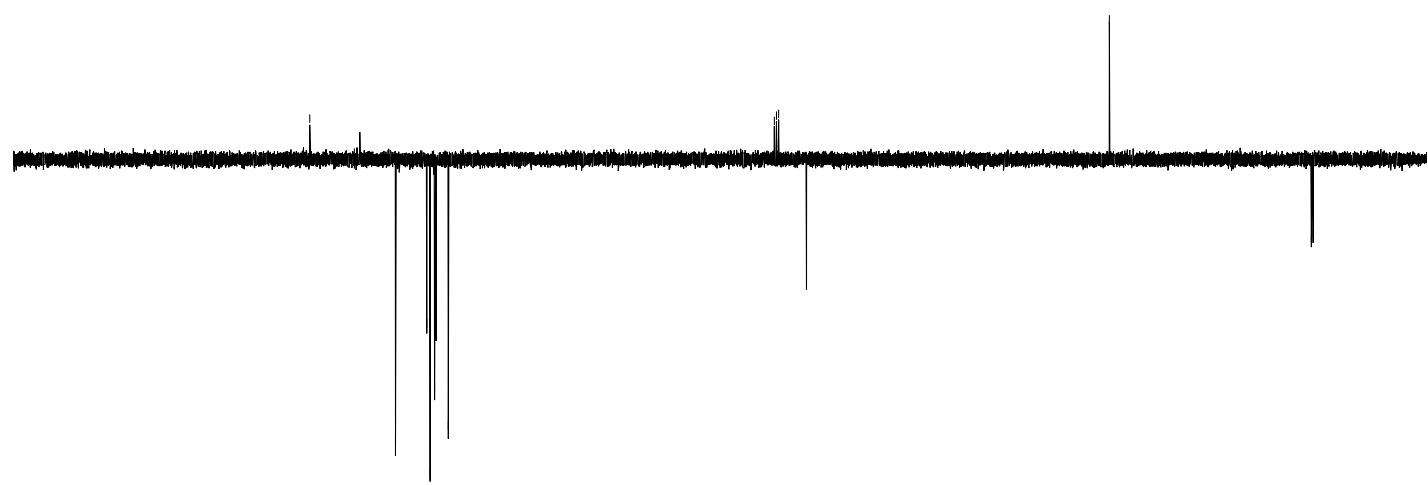

$\begin{array}{llllllllllllllllllllll}90 & 180 & 170 & 160 & 150 & 140 & 130 & 120 & 110 & 100 & 90 & 80 & 70 & 60 & 50 & 40 & 30 & 20 & 10 & 0 & -10\end{array}$ f1 (ppm) 
${ }^{1} \mathrm{H}$ NMR of 3d (400 MHZ, $\left.\mathrm{CDCl}_{3}\right)$

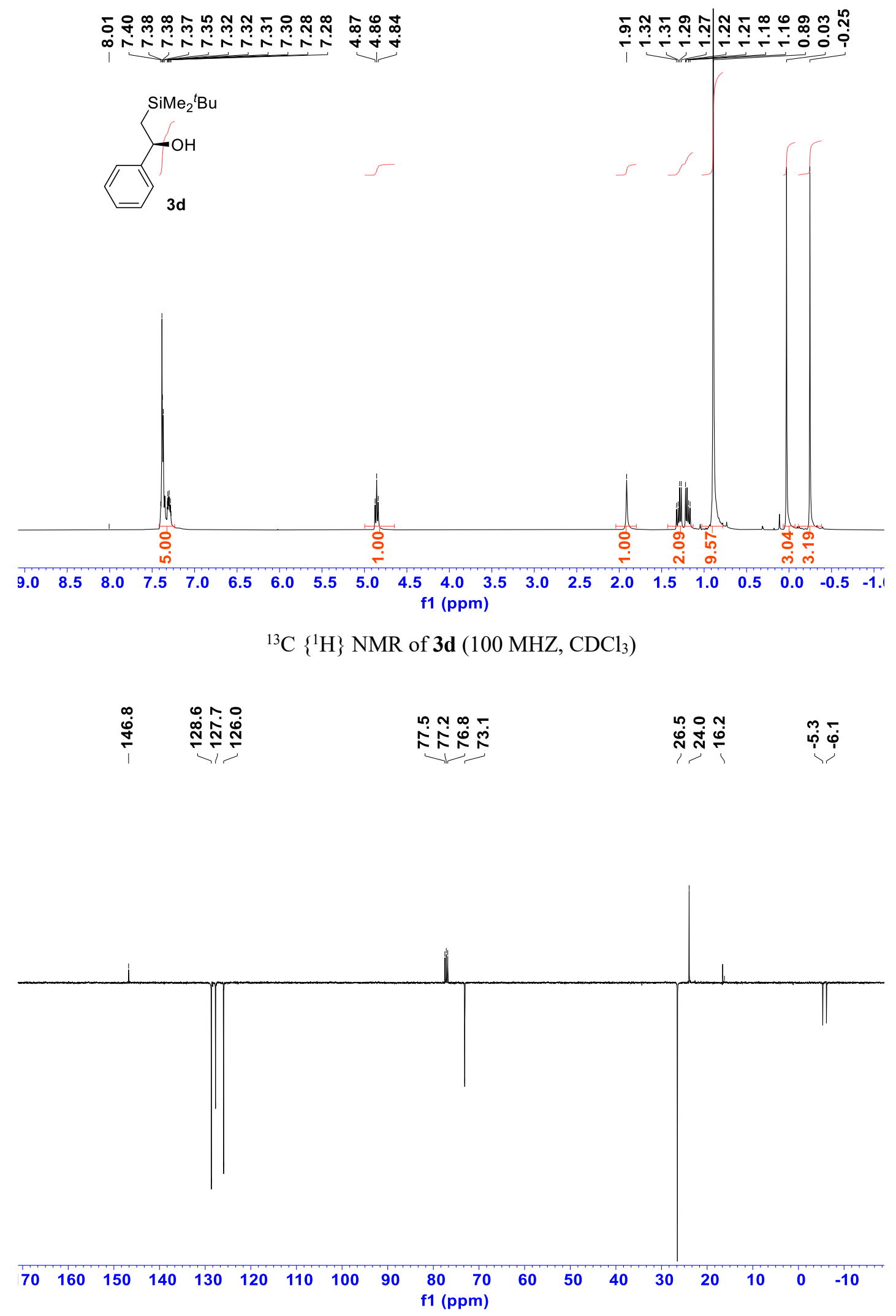


${ }^{1} \mathrm{H} \mathrm{NMR}$ of $\mathbf{3 e}\left(400 \mathrm{MHZ}, \mathrm{CDCl}_{3}\right)$

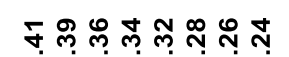

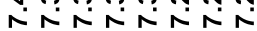

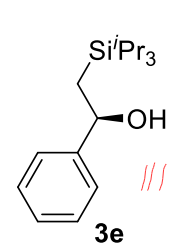

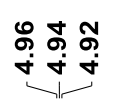

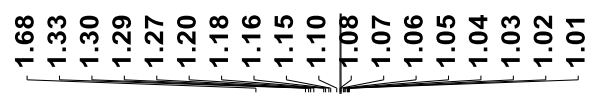

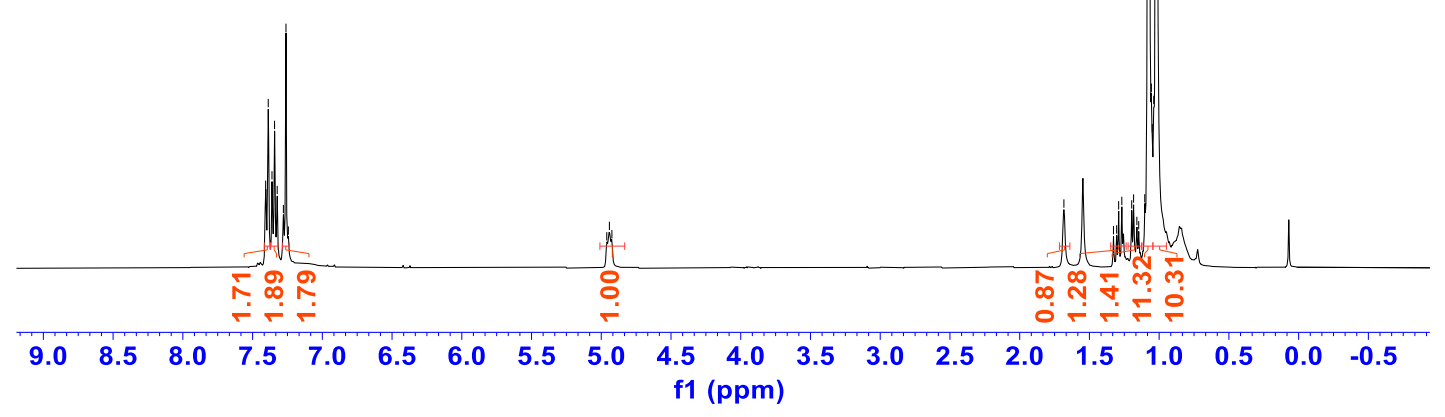

${ }^{13} \mathrm{C}\left\{{ }^{1} \mathrm{H}\right\}$ NMR of $3 \mathbf{e}\left(100 \mathrm{MHZ}, \mathrm{CDCl}_{3}\right)$

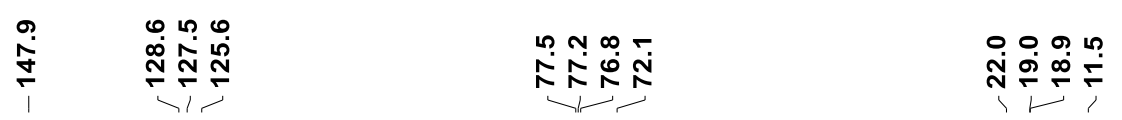

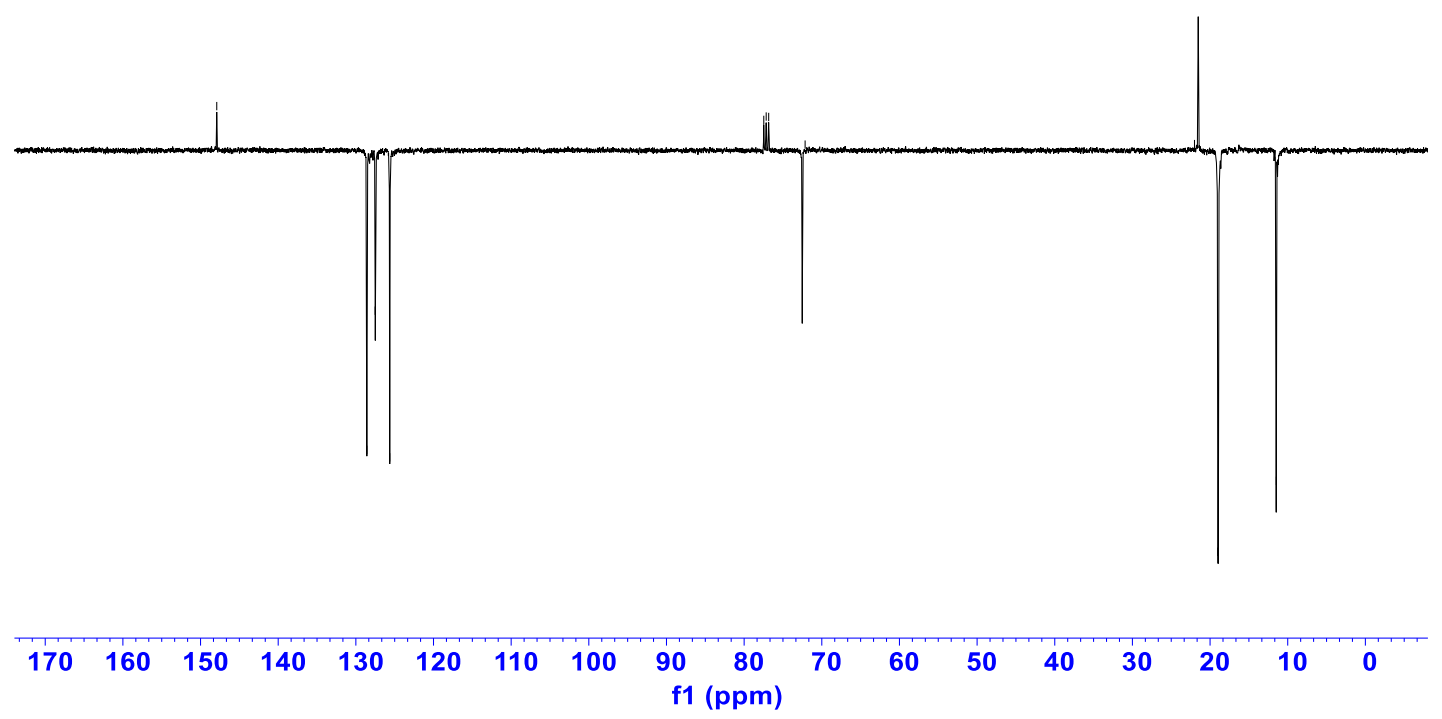


${ }^{1} \mathrm{H}$ NMR of $\mathbf{3 f}$ (400 MHZ, Acetone)

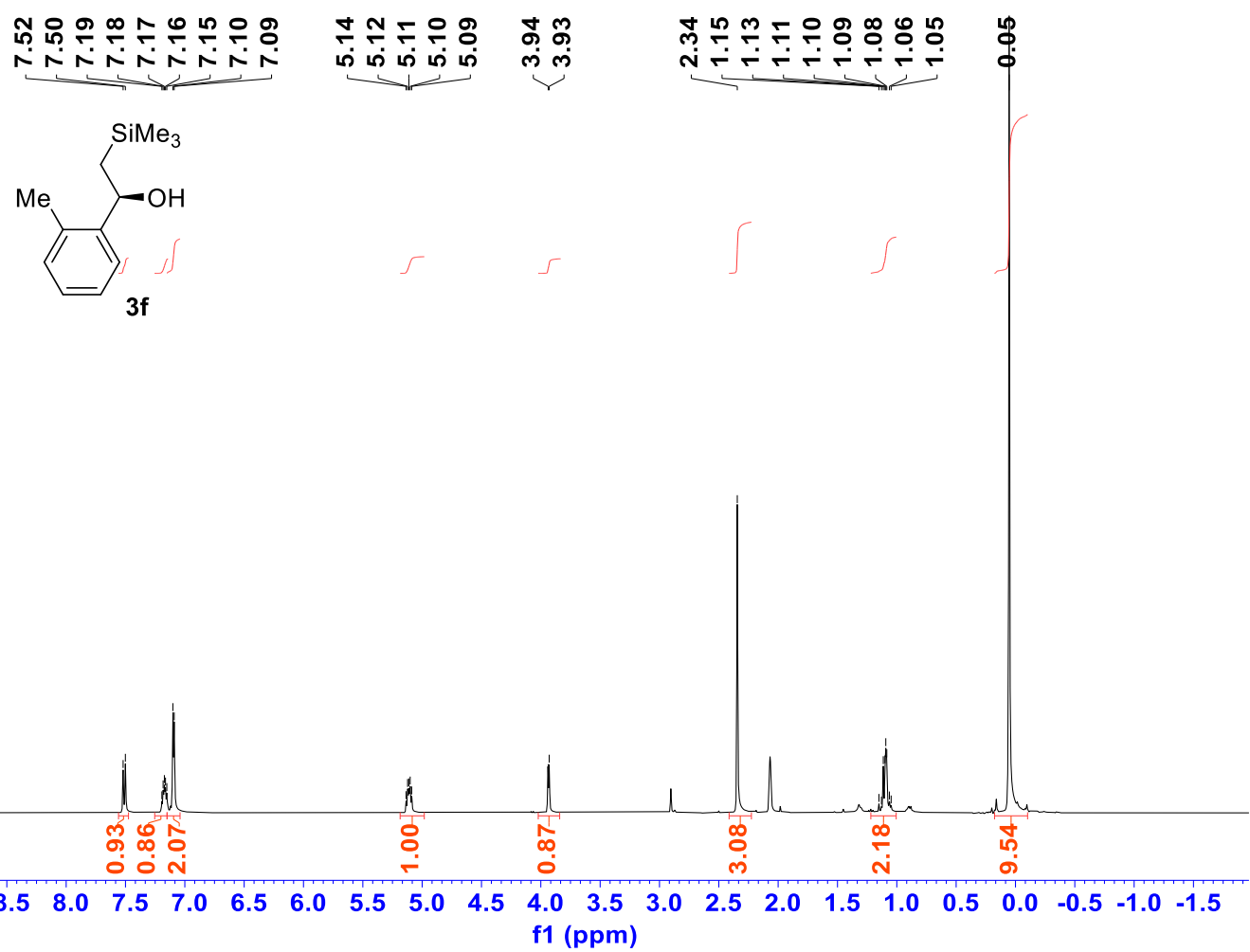

${ }^{13} \mathrm{C}\left\{{ }^{1} \mathrm{H}\right\}$ NMR of $\mathbf{3 f}(100 \mathrm{MHZ}$, Acetone)

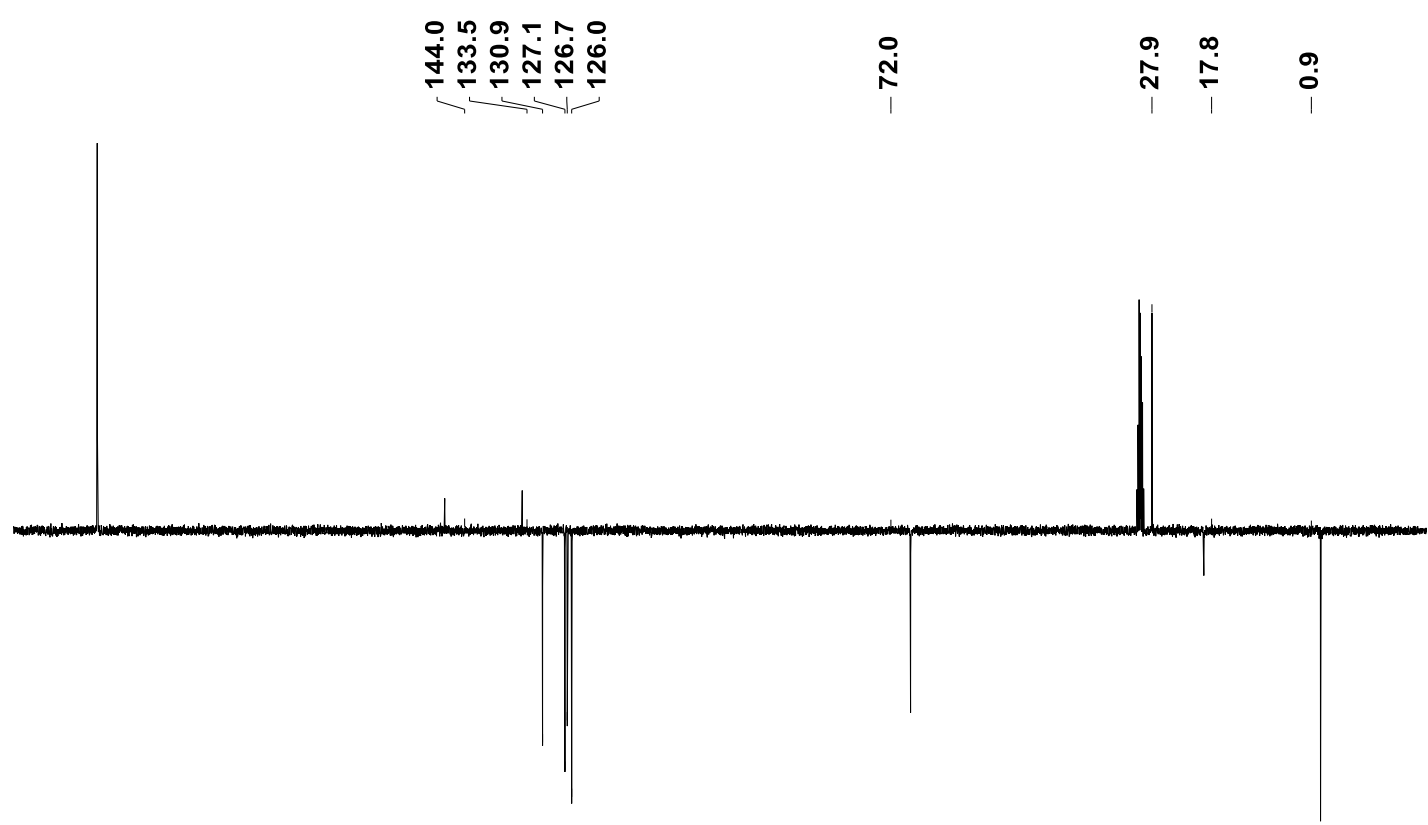

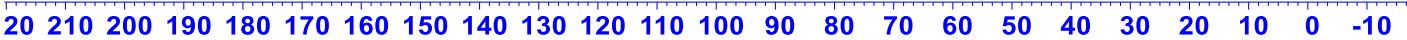
f1 (ppm) 
${ }^{1} \mathrm{H}$ NMR of 3g (400 MHZ, Acetone)

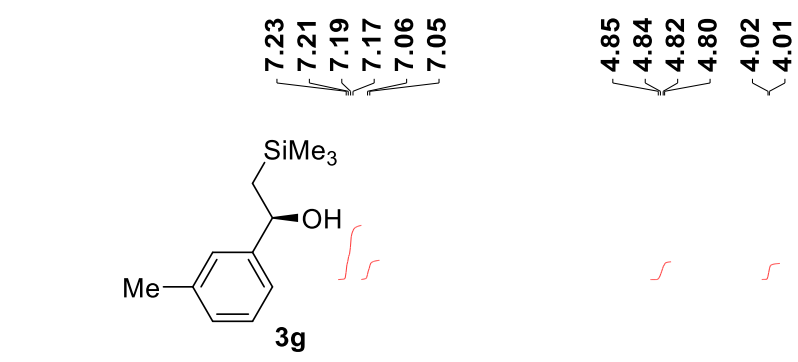

象 点

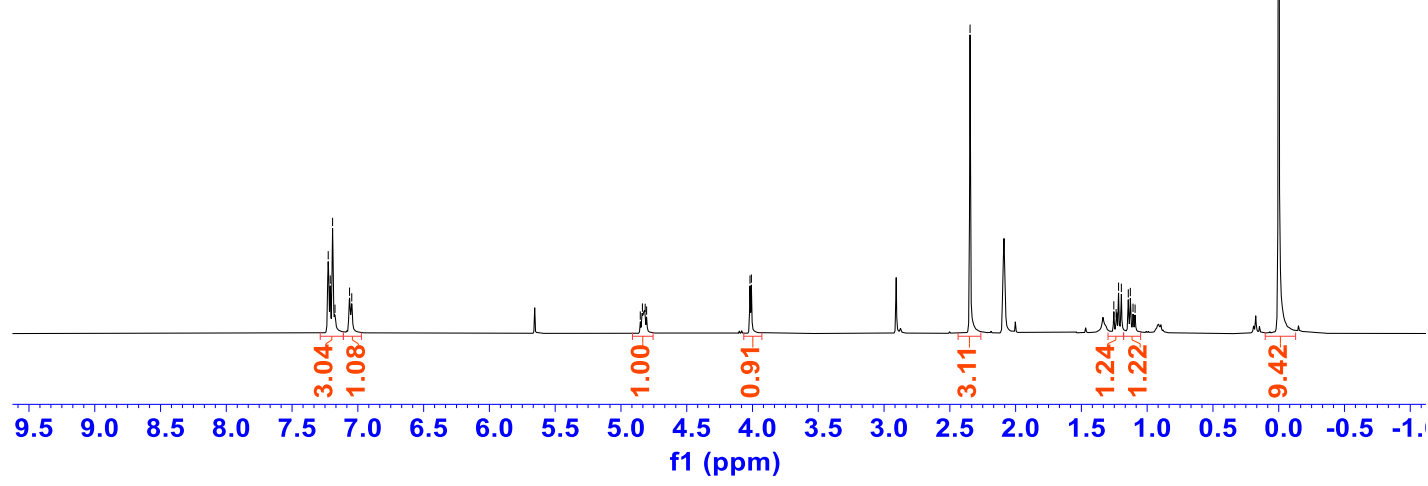

${ }^{13} \mathrm{C}\left\{{ }^{1} \mathrm{H}\right\}$ NMR of $\mathbf{3 g}$ (100 MHZ, Acetone)

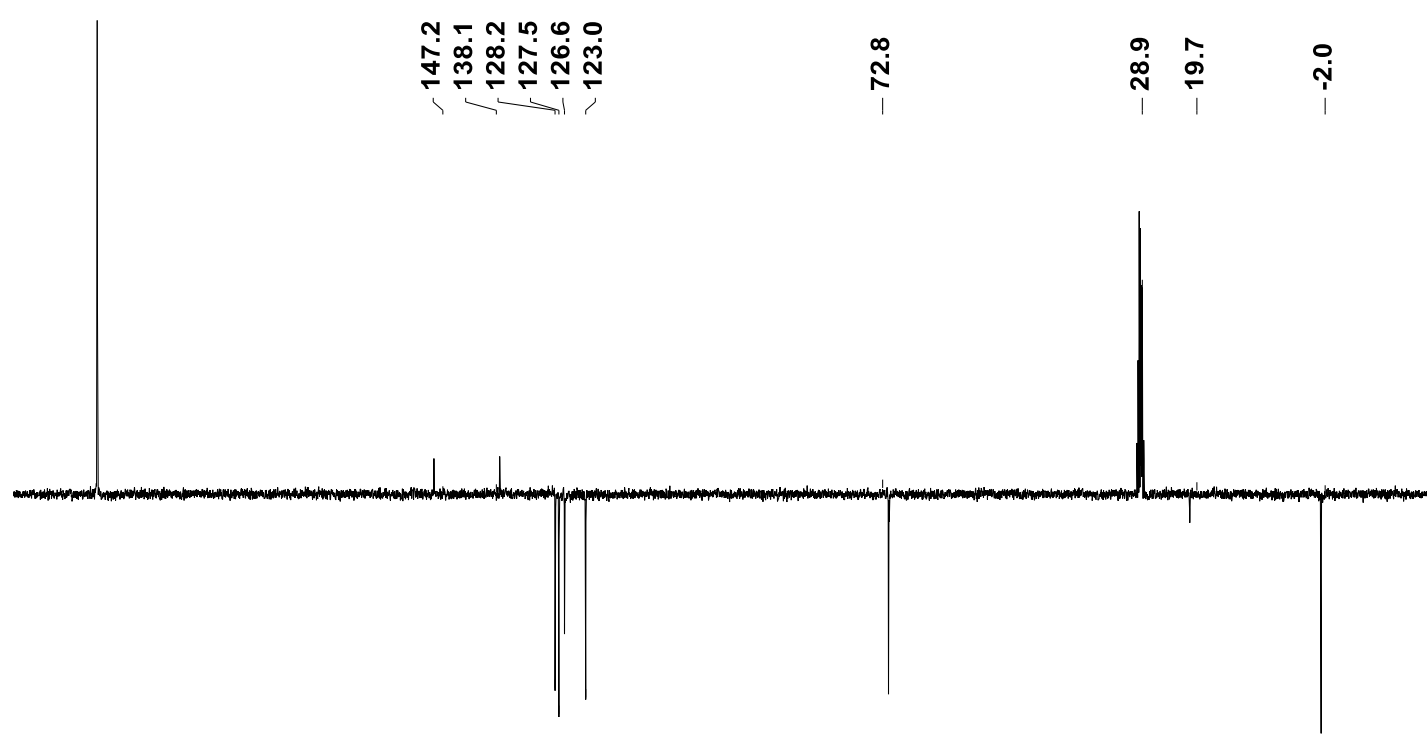

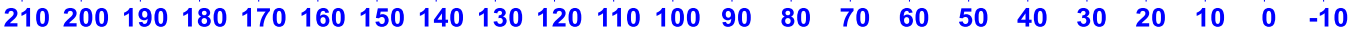
f1 (ppm) 
${ }^{1} \mathrm{H}$ NMR of $3 \mathbf{h}$ (400 MHZ, Acetone)

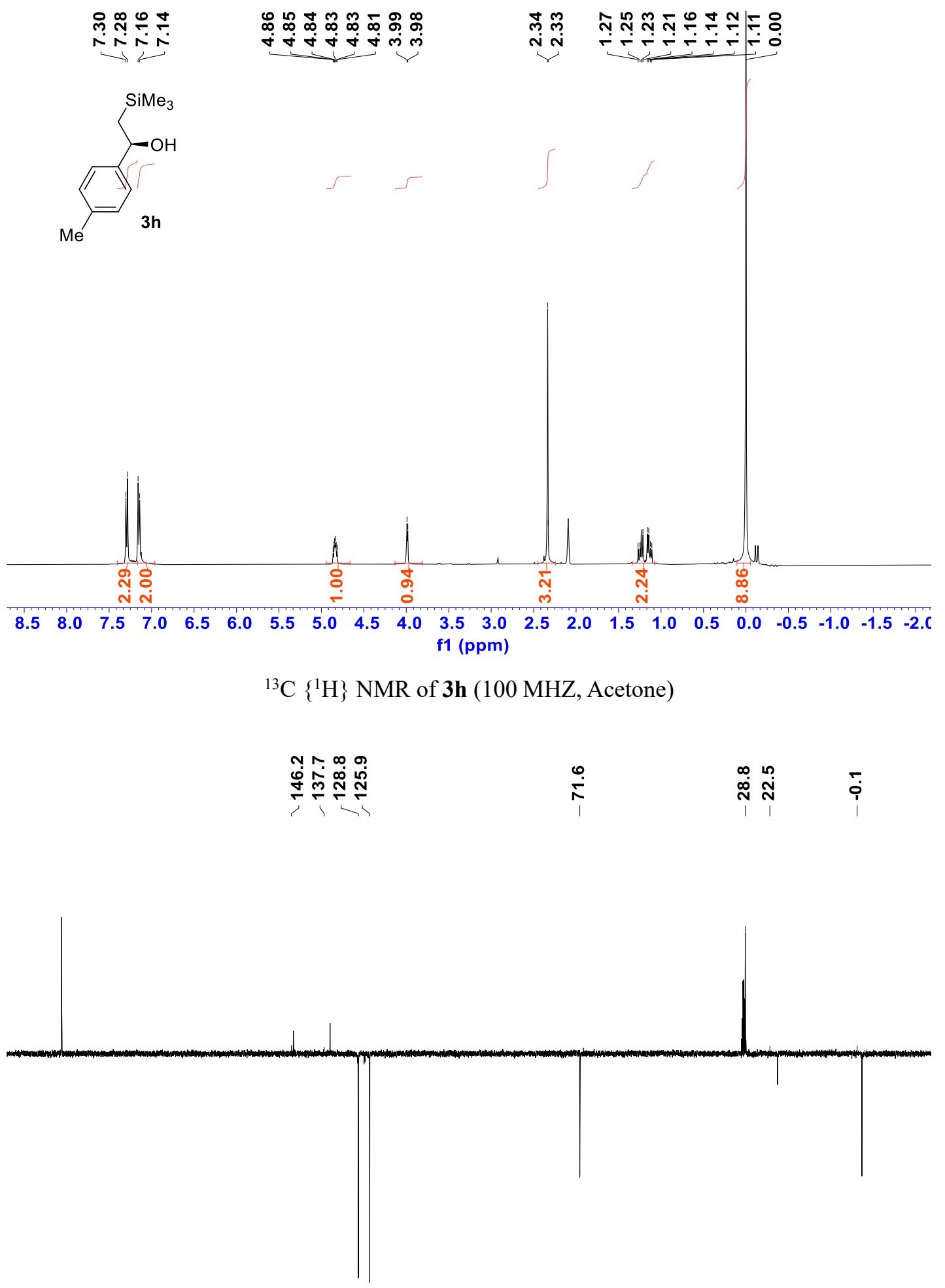

$210200190180170160150140130120110100 \quad 90 \quad 80 \quad 70 \quad 60 \begin{array}{llllllllll}50 & 40 & 30 & 20 & 10 & 0 & -10\end{array}$ f1 (ppm) 
${ }^{1} \mathrm{H}$ NMR of 3i (400 MHZ, Acetone)

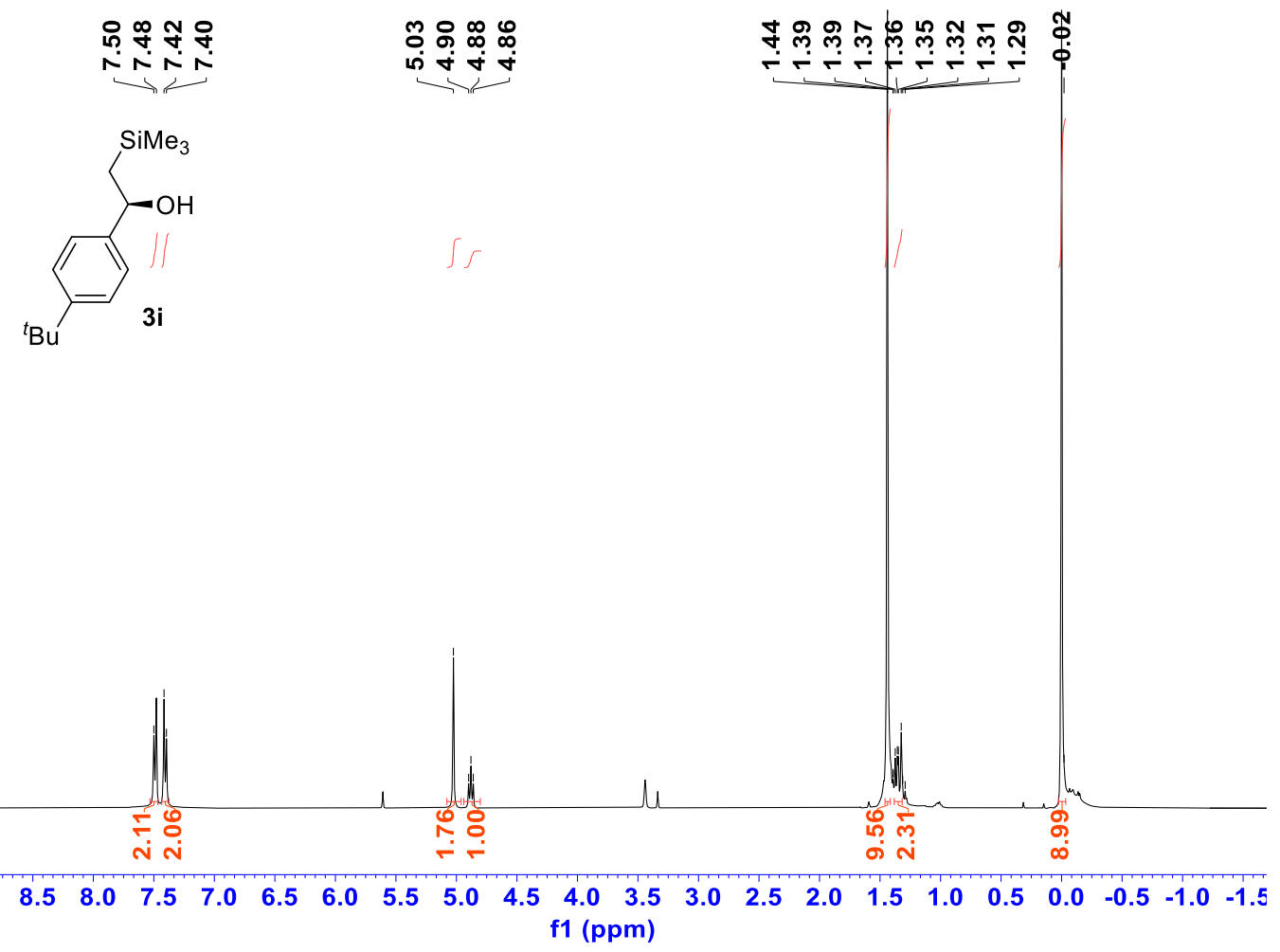

${ }^{13} \mathrm{C}\left\{{ }^{1} \mathrm{H}\right\}$ NMR of $3 \mathbf{i}(100 \mathrm{MHZ}$, Acetone)

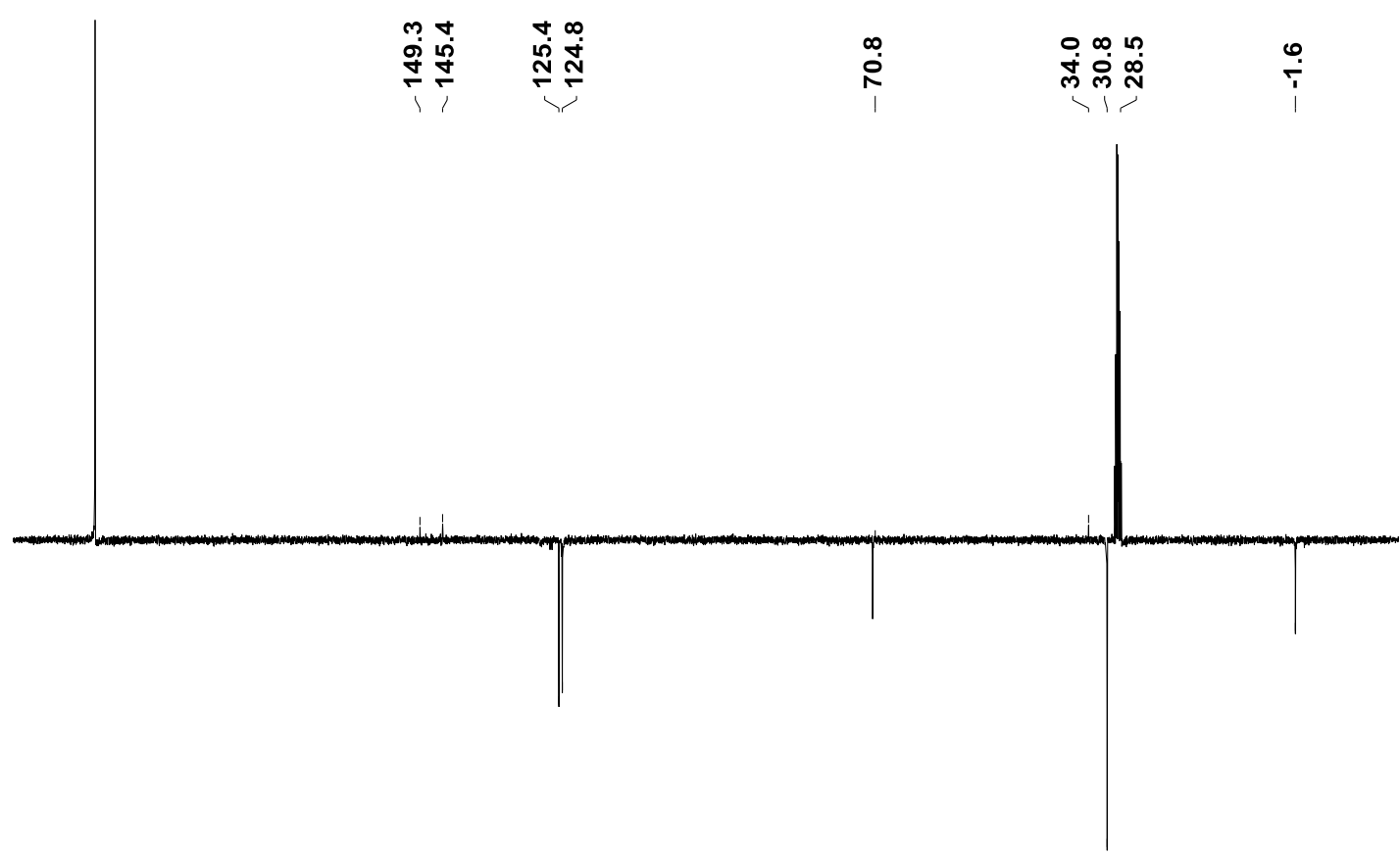

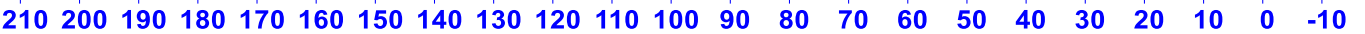
f1 (ppm) 
${ }^{1} \mathrm{H}$ NMR of $\mathbf{3 j}$ (400 MHZ, Acetone)

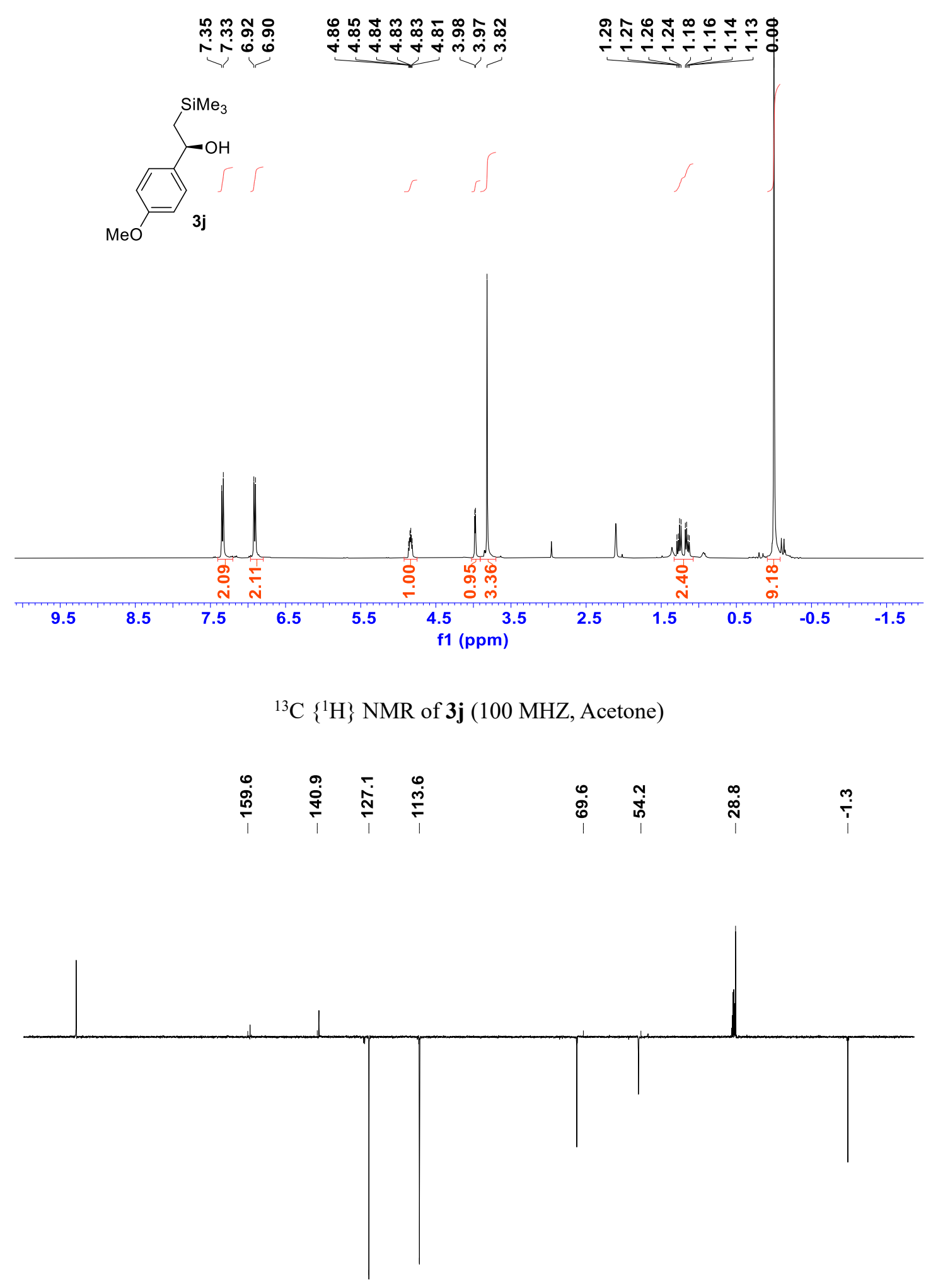

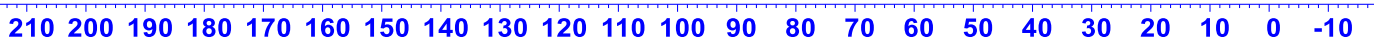
f1 (ppm) 
${ }^{1} \mathrm{H}$ NMR of 3k (400 MHZ, Acetone)
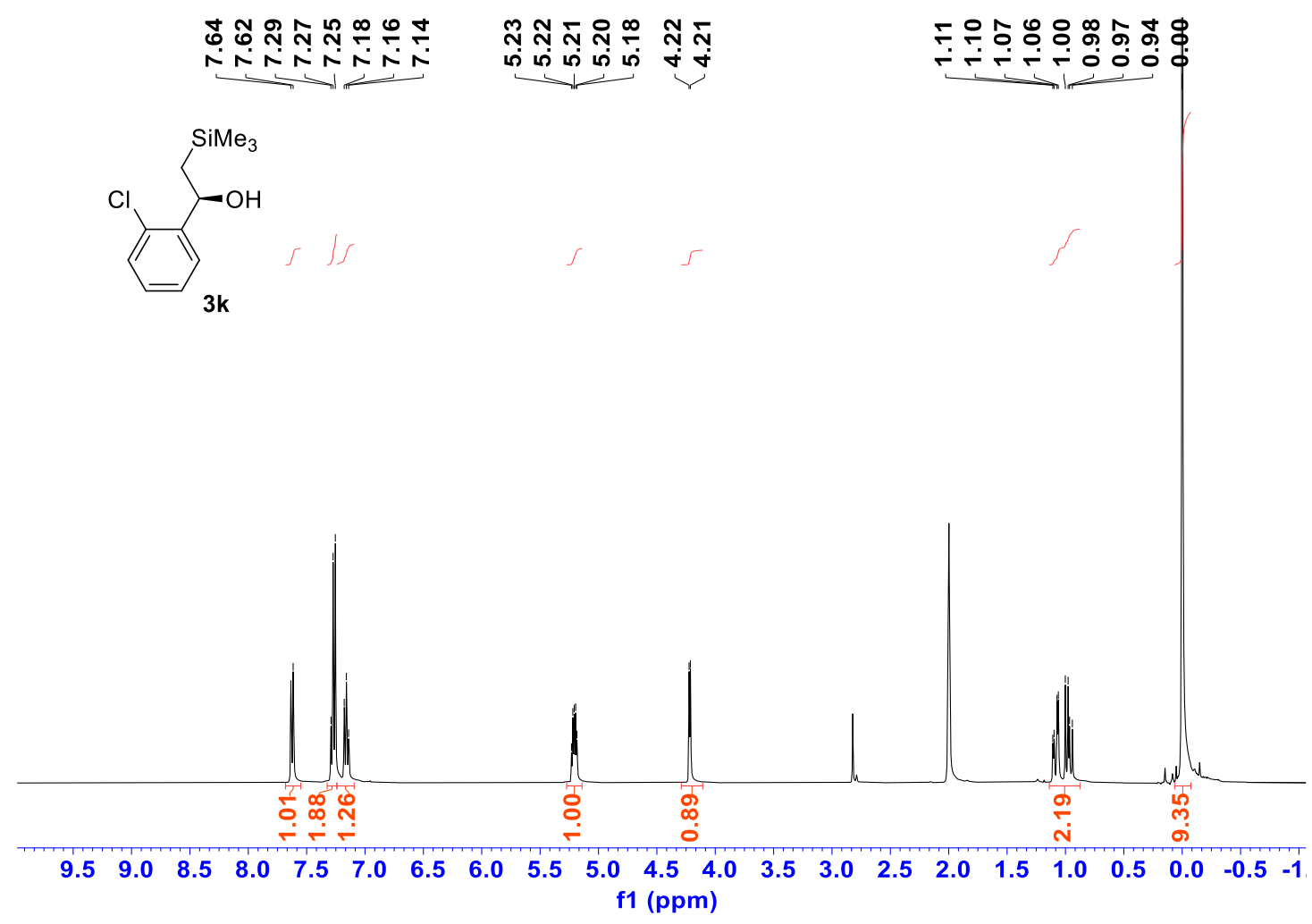

${ }^{13} \mathrm{C}\left\{{ }^{1} \mathrm{H}\right\}$ NMR of 3k (100 MHZ, Acetone)

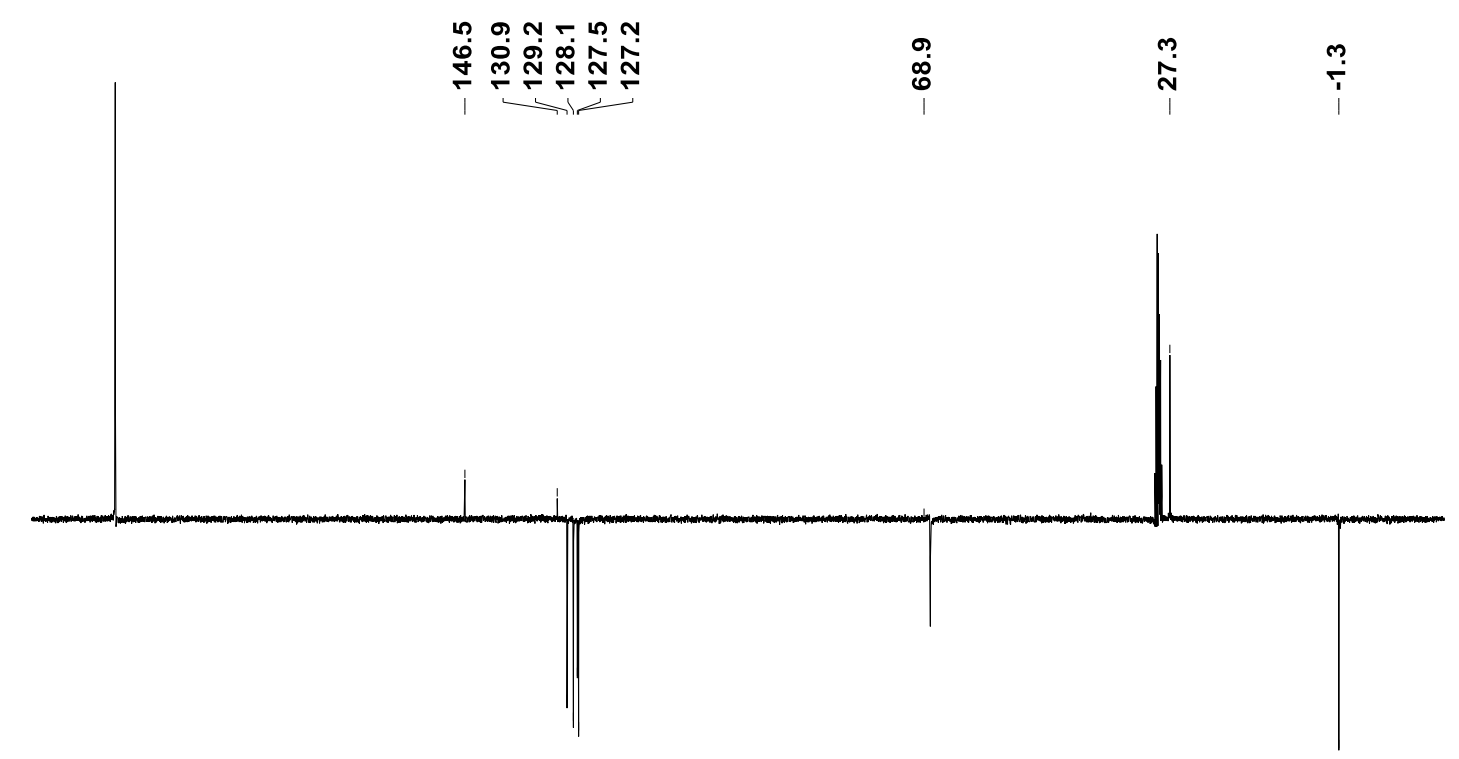

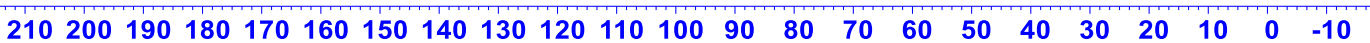
f1 (ppm) 
${ }^{1} \mathrm{H}$ NMR of 31 (400 MHZ, $\mathrm{CDCl}_{3}$ )

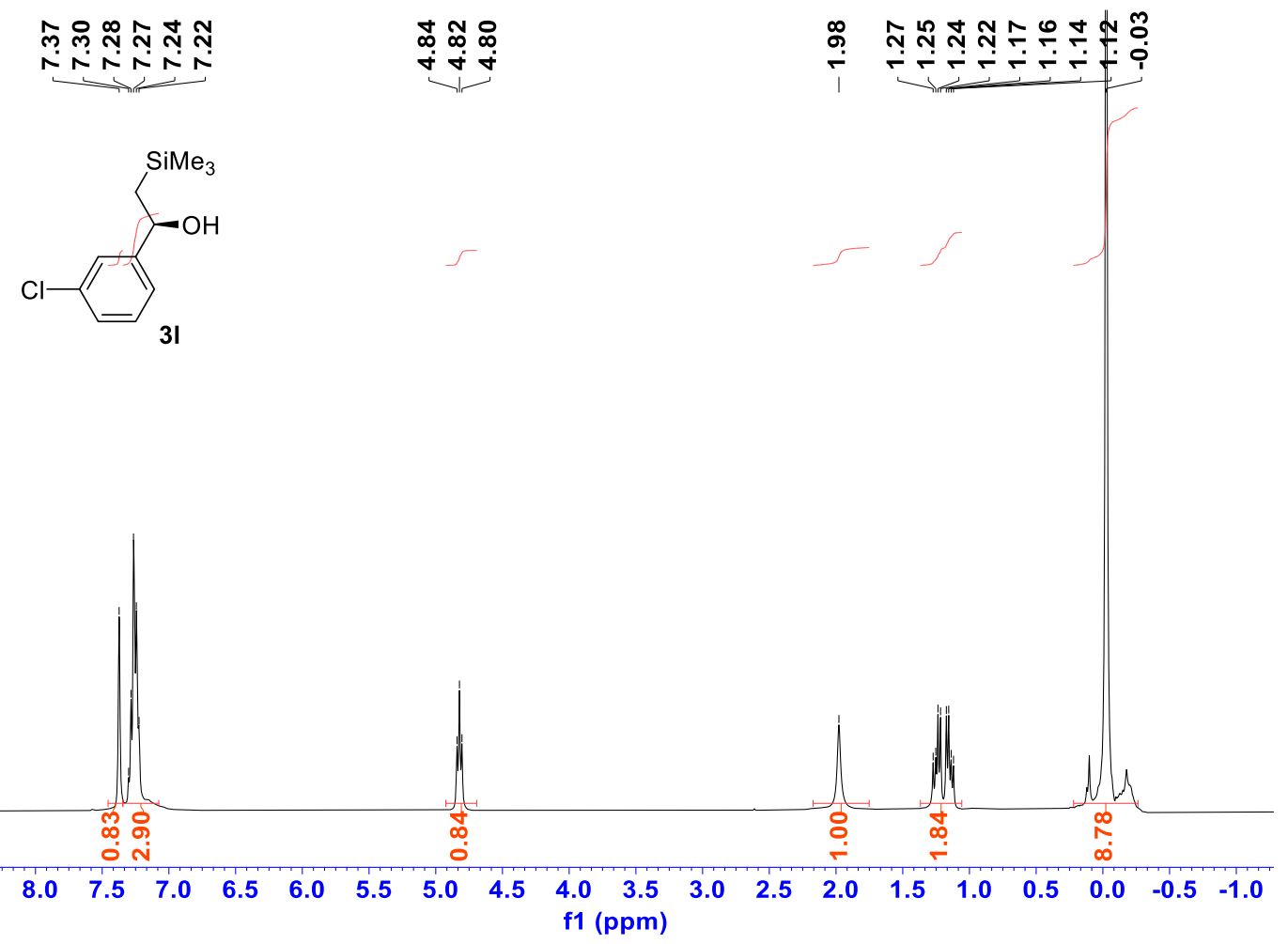

${ }^{13} \mathrm{C}\left\{{ }^{1} \mathrm{H}\right\}$ NMR of $31\left(100 \mathrm{MHZ}, \mathrm{CDCl}_{3}\right)$
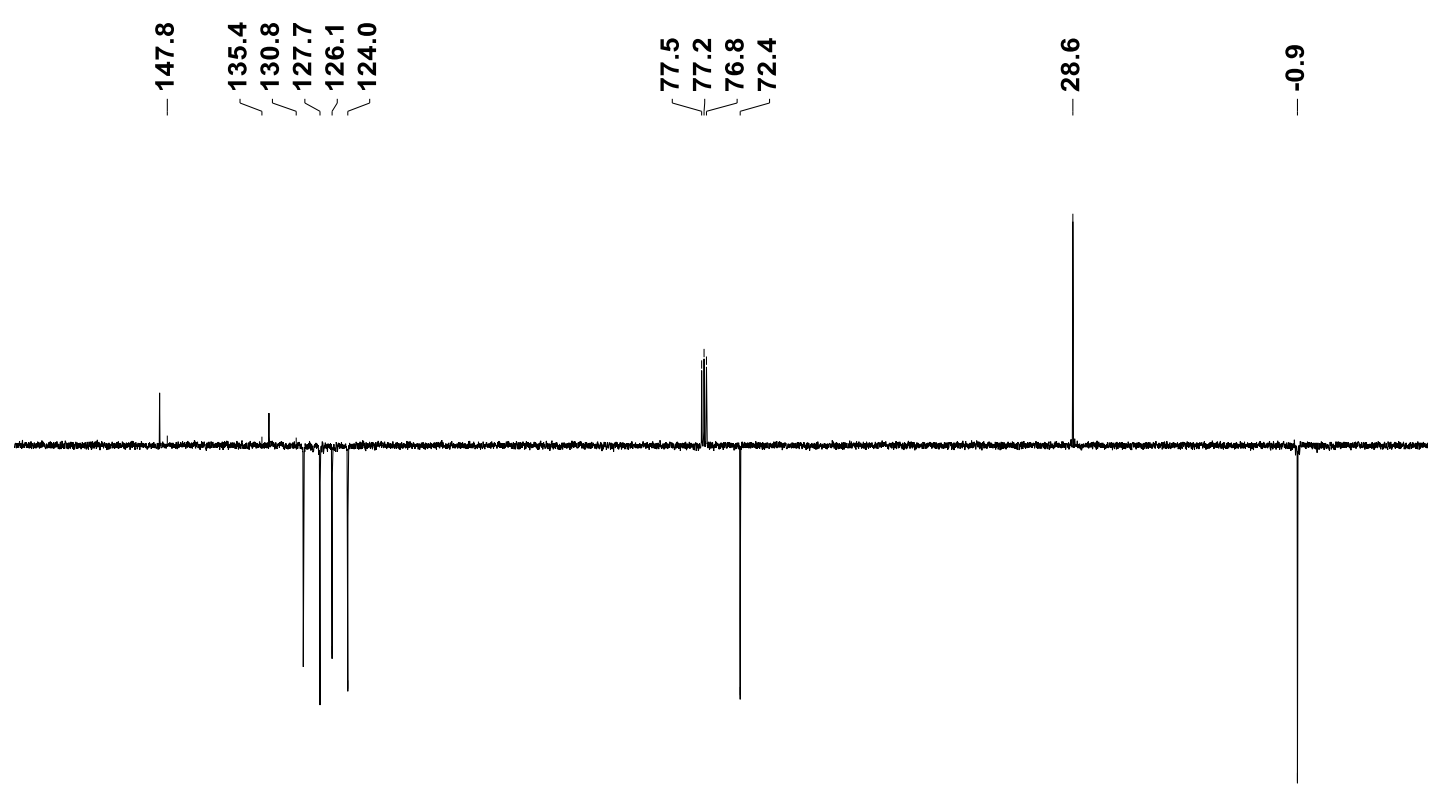

$\begin{array}{llllllllllllllllll}160 & 150 & 140 & 130 & 120 & 110 & 100 & 90 & \begin{array}{l}80 \\ \mathrm{f} 1(\mathrm{ppm})\end{array} & 70 & 60 & 50 & 40 & 30 & 20 & 10 & 0 & -10\end{array}$ 
${ }^{1} \mathrm{H}$ NMR of $\mathbf{3 m}\left(400 \mathrm{MHZ}, \mathrm{CDCl}_{3}\right)$

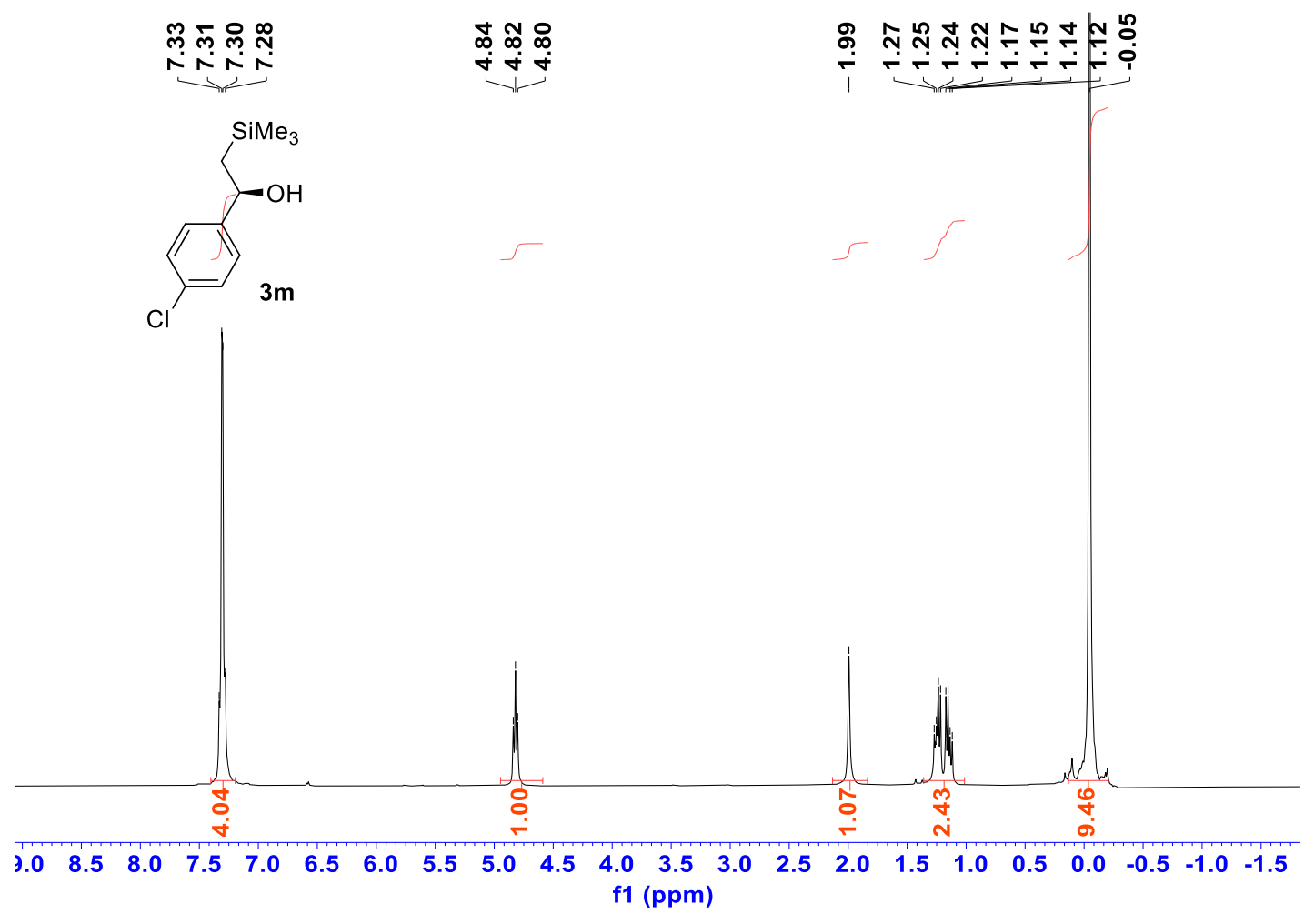

${ }^{13} \mathrm{C}\left\{{ }^{1} \mathrm{H}\right\}$ NMR of $\mathbf{3 m}\left(100 \mathrm{MHZ}, \mathrm{CDCl}_{3}\right)$

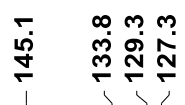

กீำ

k下in

$\stackrel{\infty}{\text { ஸे }}$

$\bar{i}$

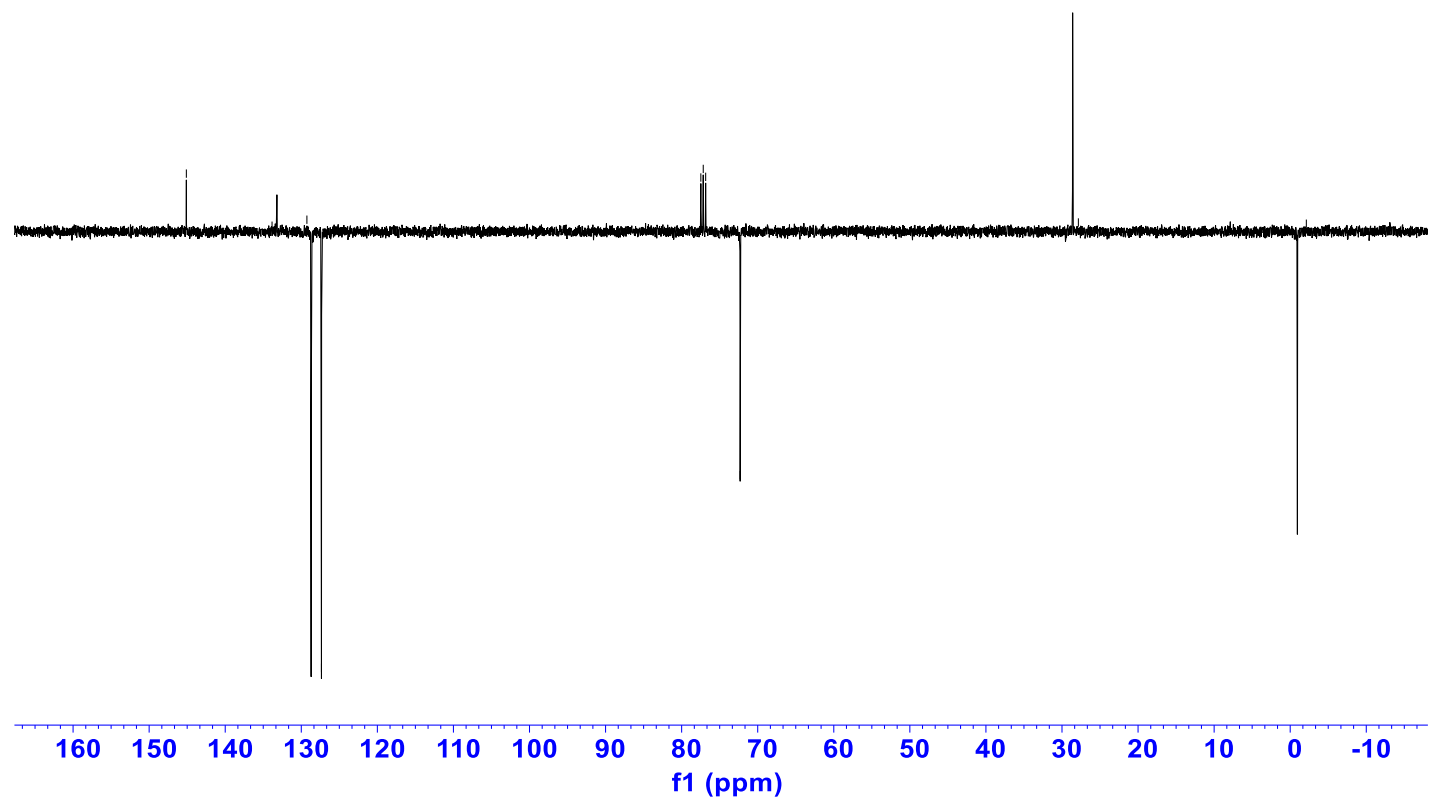


${ }^{1} \mathrm{H}$ NMR of 3n (400 MHZ, $\left.\mathrm{CDCl}_{3}\right)$
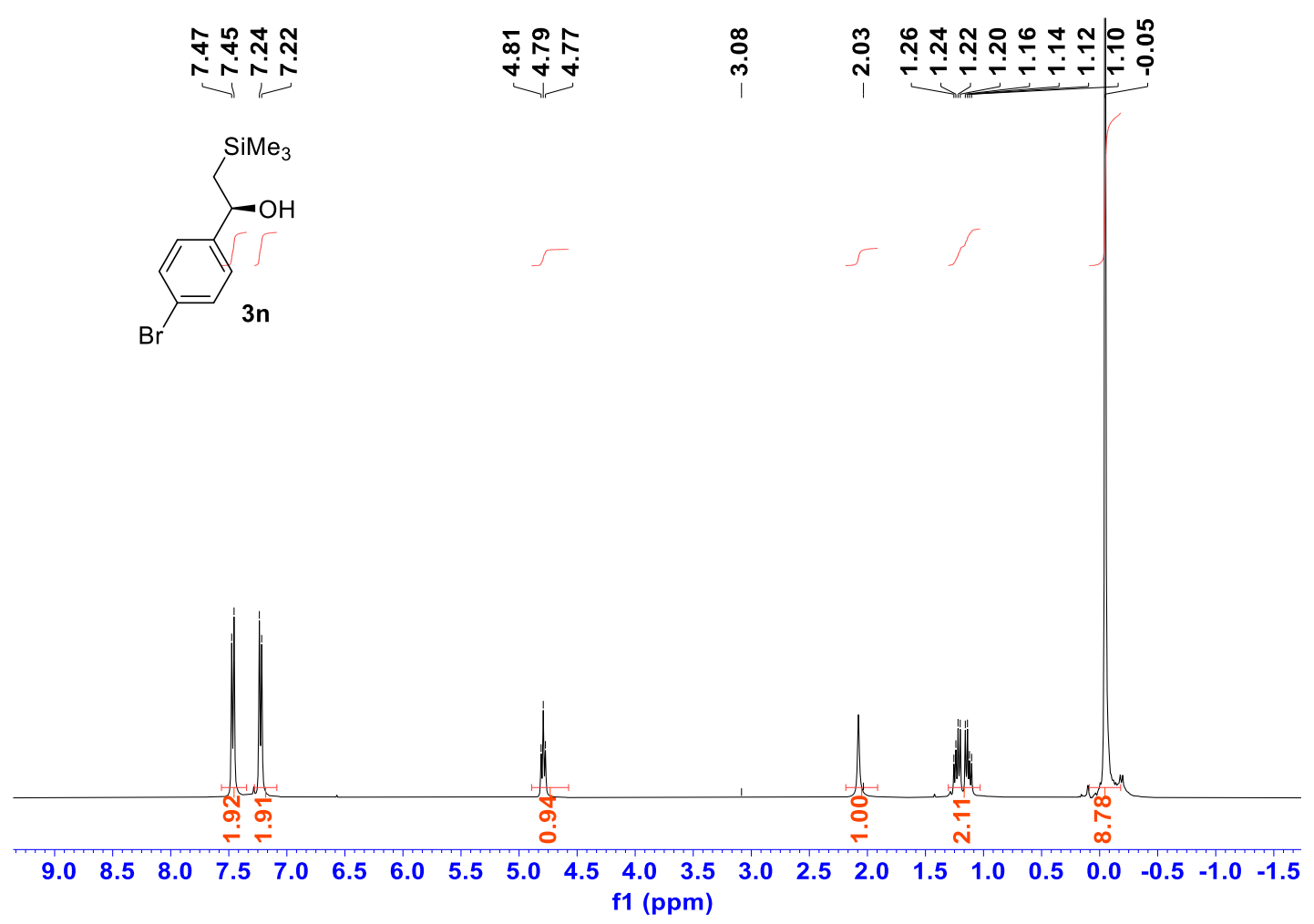

${ }^{13} \mathrm{C}\left\{{ }^{1} \mathrm{H}\right\}$ NMR of $\mathbf{3 n}\left(100 \mathrm{MHZ}, \mathrm{CDCl}_{3}\right)$

\begin{tabular}{|c|c|c|}
\hline 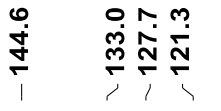 & 占 & $\stackrel{\text { L }}{\underset{\infty}{\text { d }}}$ \\
\hline
\end{tabular}

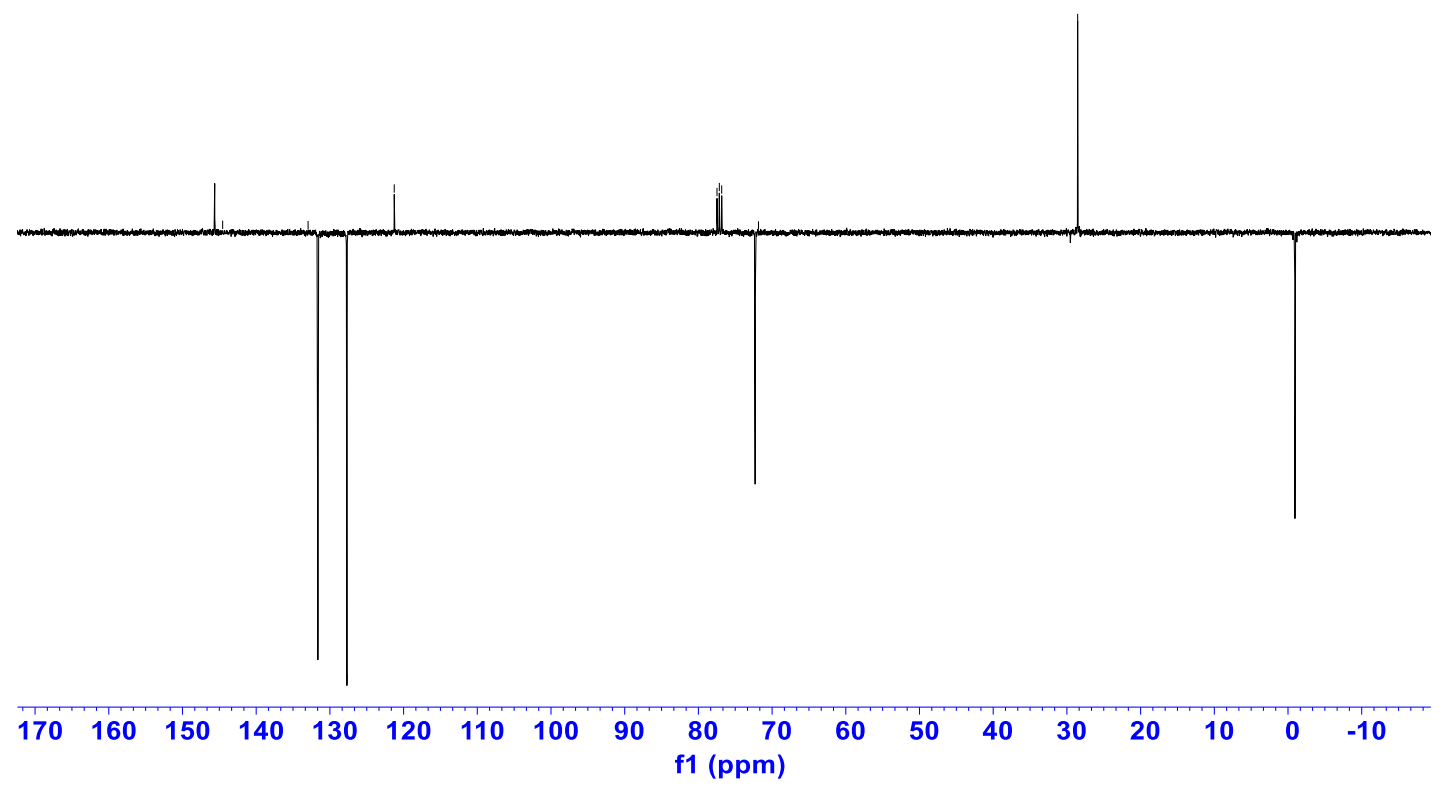


${ }^{1} \mathrm{H}$ NMR of $3 \mathbf{o}\left(400 \mathrm{MHZ}, \mathrm{CDCl}_{3}\right)$

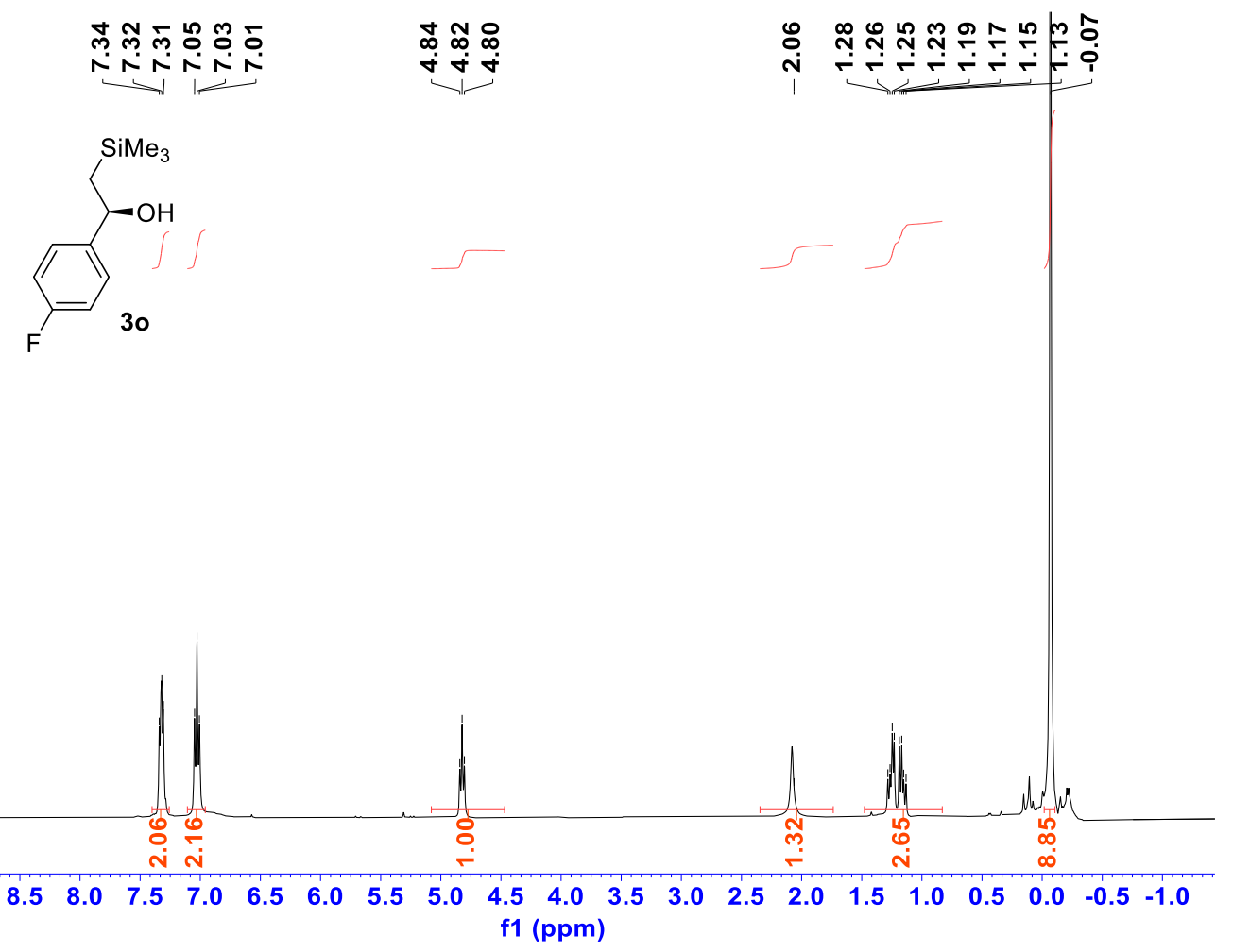

${ }^{13} \mathrm{C}\left\{{ }^{1} \mathrm{H}\right\}$ NMR of $30\left(100 \mathrm{MHZ}, \mathrm{CDCl}_{3}\right)$

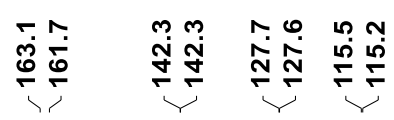

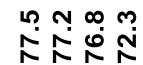

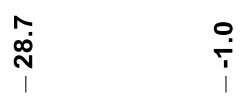

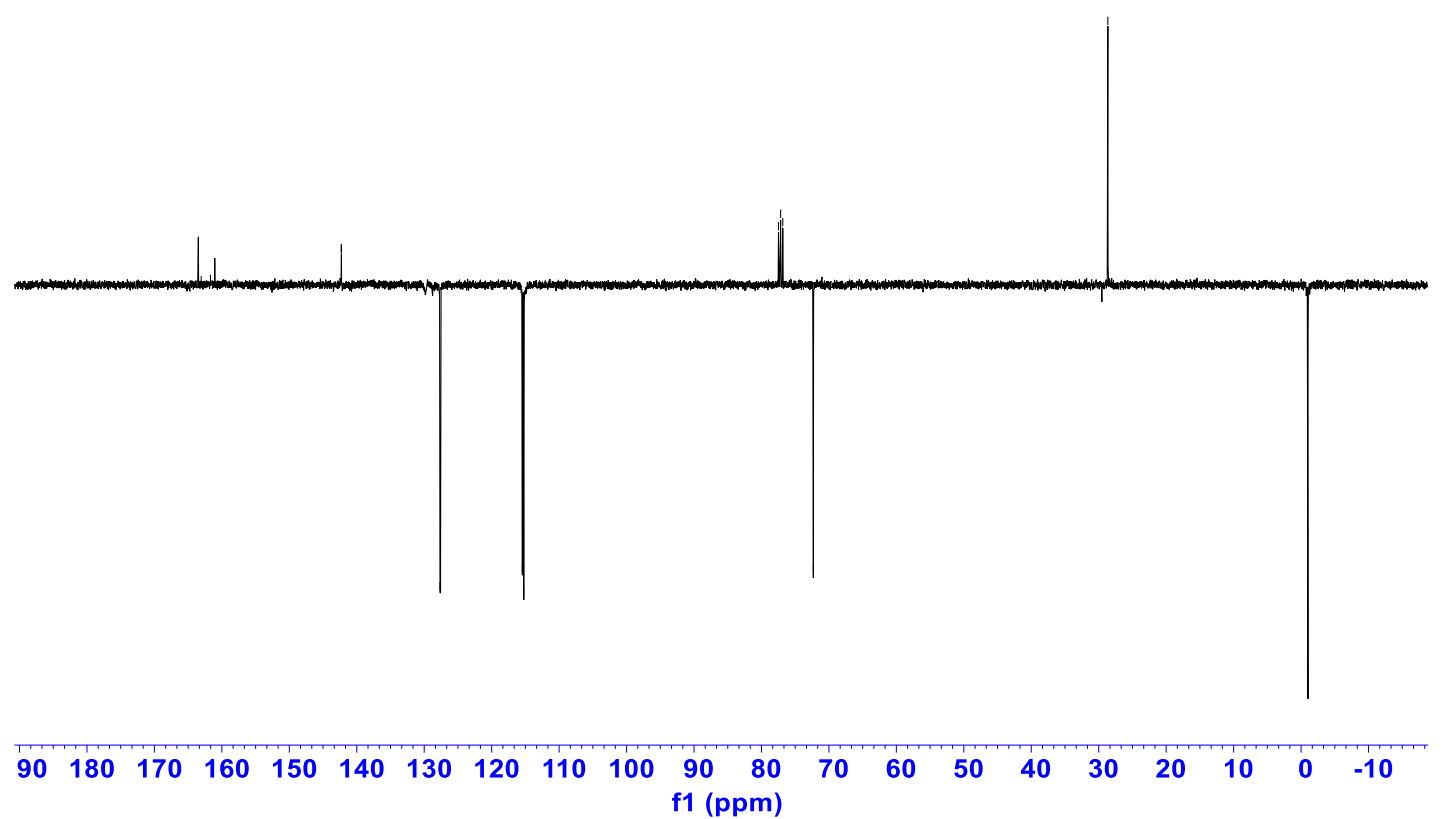


${ }^{19} \mathrm{~F}$ NMR of $3 \mathbf{0}\left(377 \mathrm{MHZ}, \mathrm{CDCl}_{3}\right)$

움

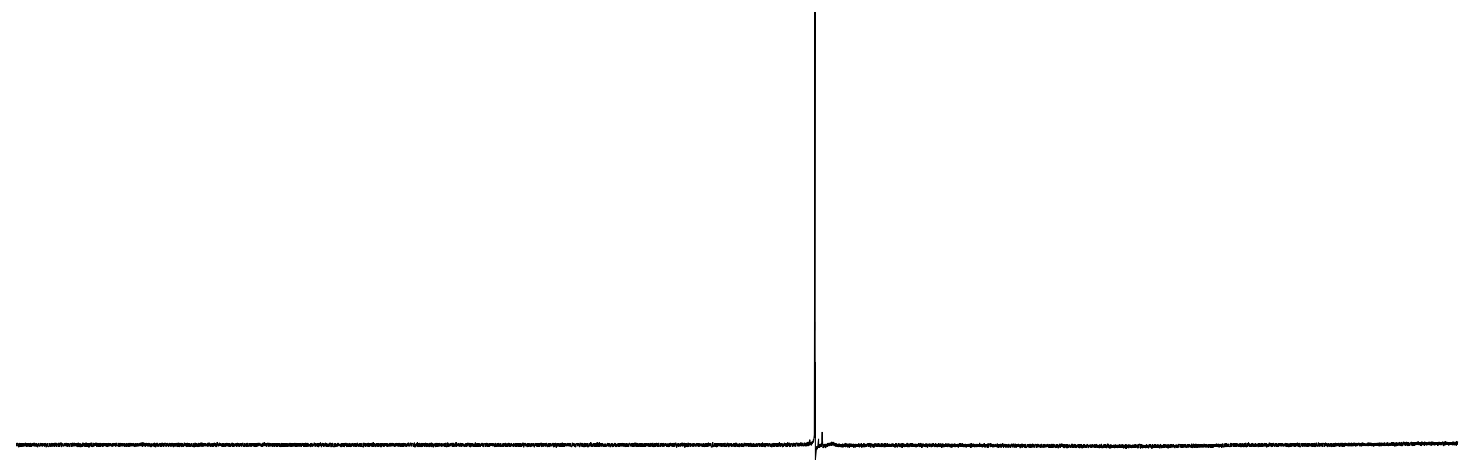

\begin{tabular}{llllllllllllllllllll}
\hline 10 & -20 & -30 & -40 & -50 & -60 & -70 & -80 & -90 & -100 & -110 & -120 & -130 & -140 & -150 & -160 & -170 & -180 & -190 & -2
\end{tabular} f1 (ppm) 
${ }^{1} \mathrm{H}$ NMR of $\mathbf{3 p}\left(400 \mathrm{MHZ}, \mathrm{CDCl}_{3}\right)$
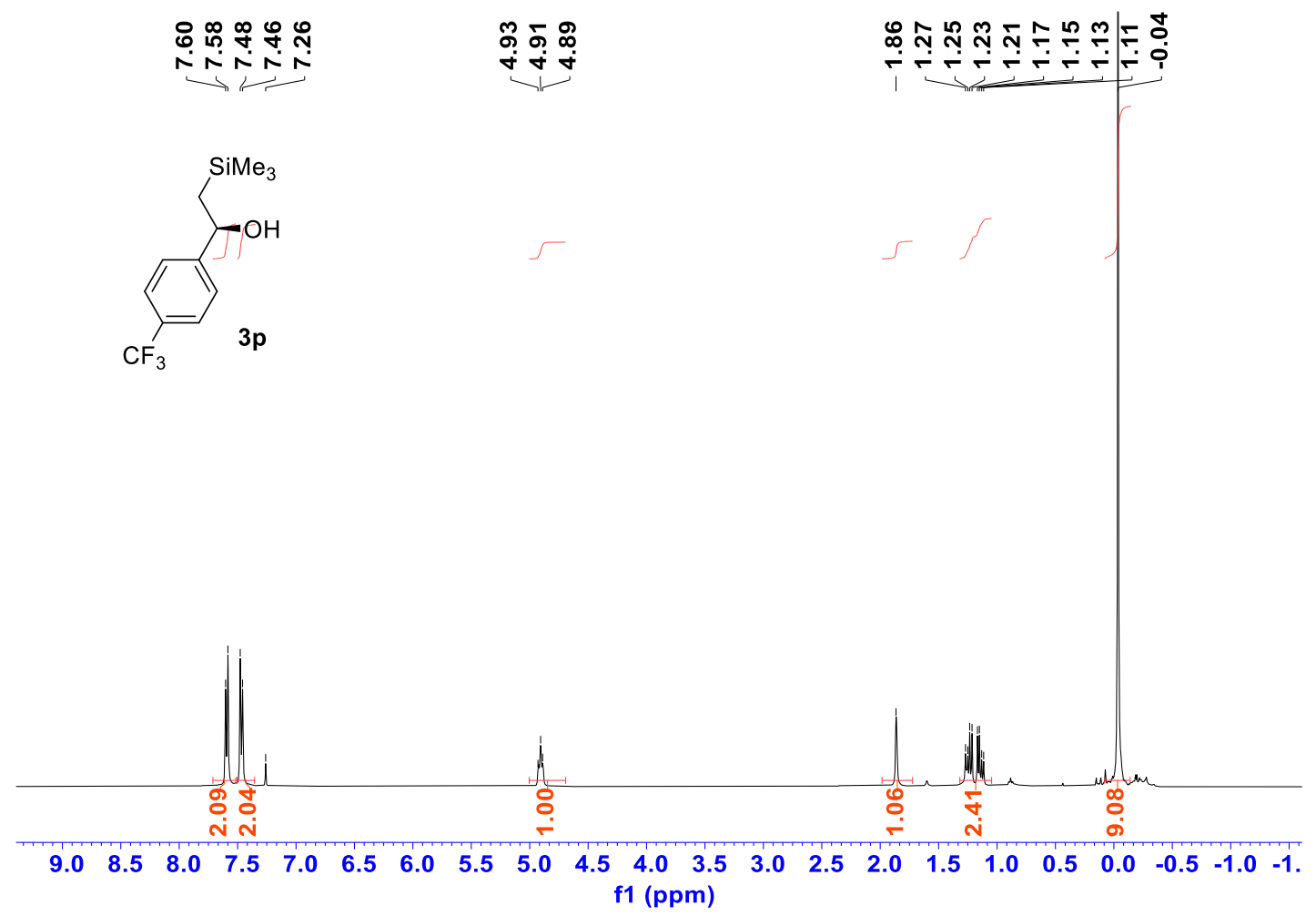

${ }^{13} \mathrm{C}\left\{{ }^{1} \mathrm{H}\right\}$ NMR of $\mathbf{3 p}\left(100 \mathrm{MHZ}, \mathrm{CDCl}_{3}\right)$

官
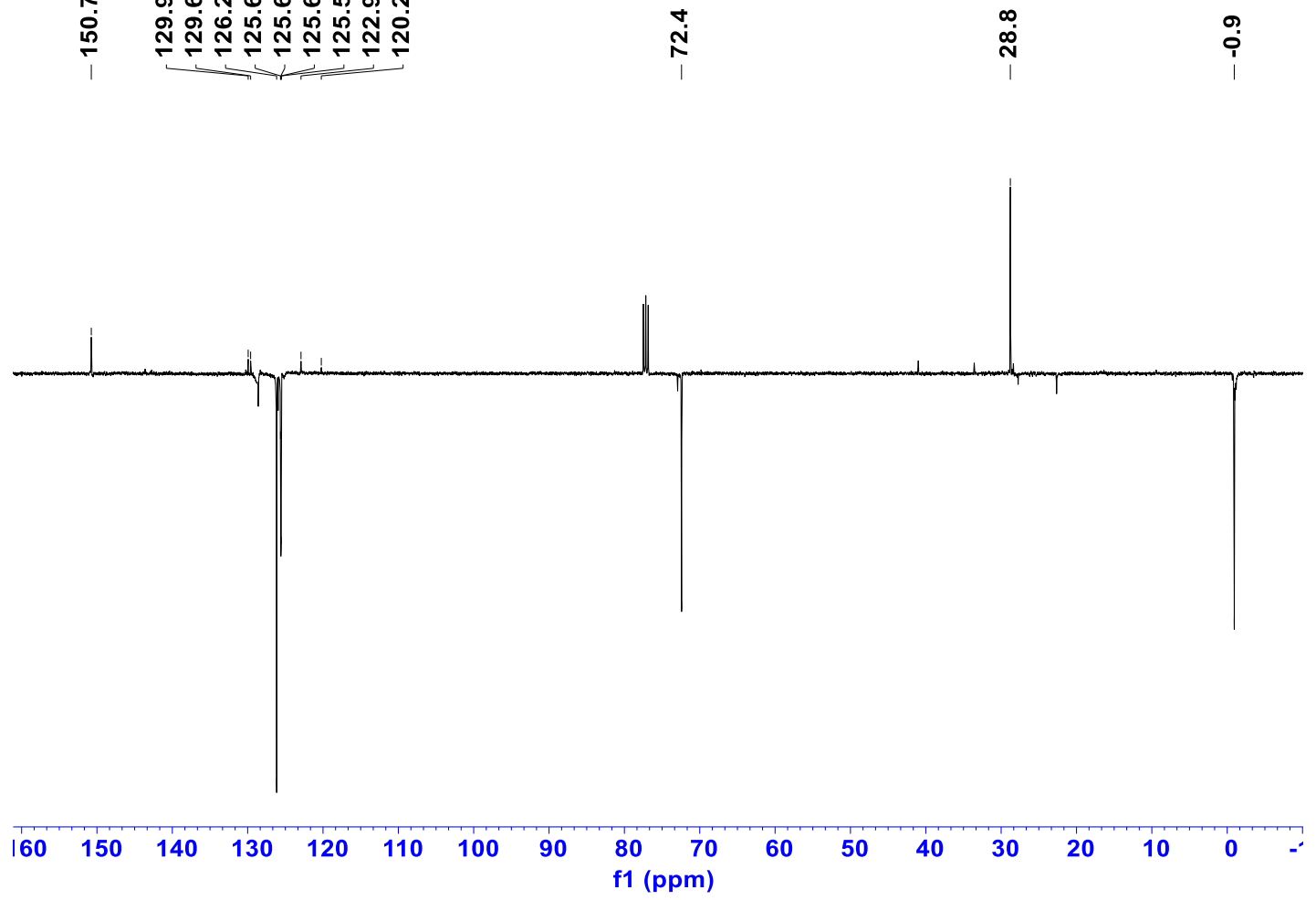
${ }^{19} \mathrm{~F}$ NMR of $3 \mathbf{p}$ (377 MHZ, $\mathrm{CDCl}_{3}$

彳े

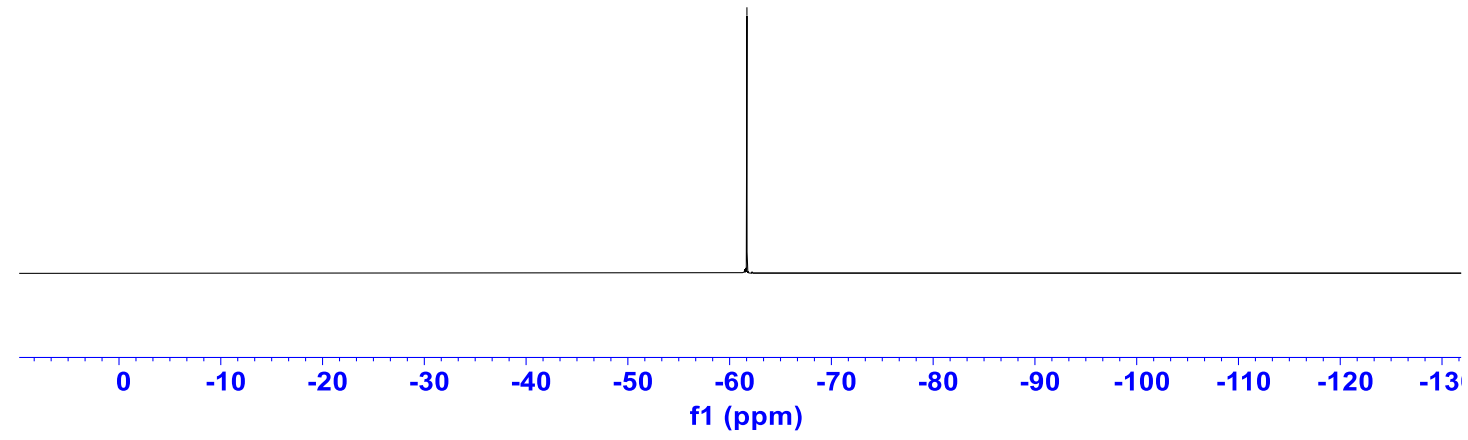


${ }^{1} \mathrm{H}$ NMR of 3q (400 MHZ, Acetone)

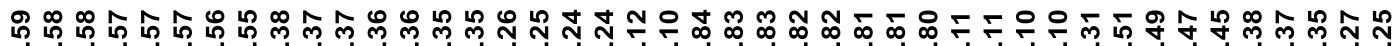

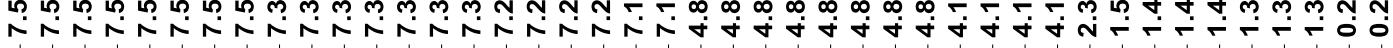
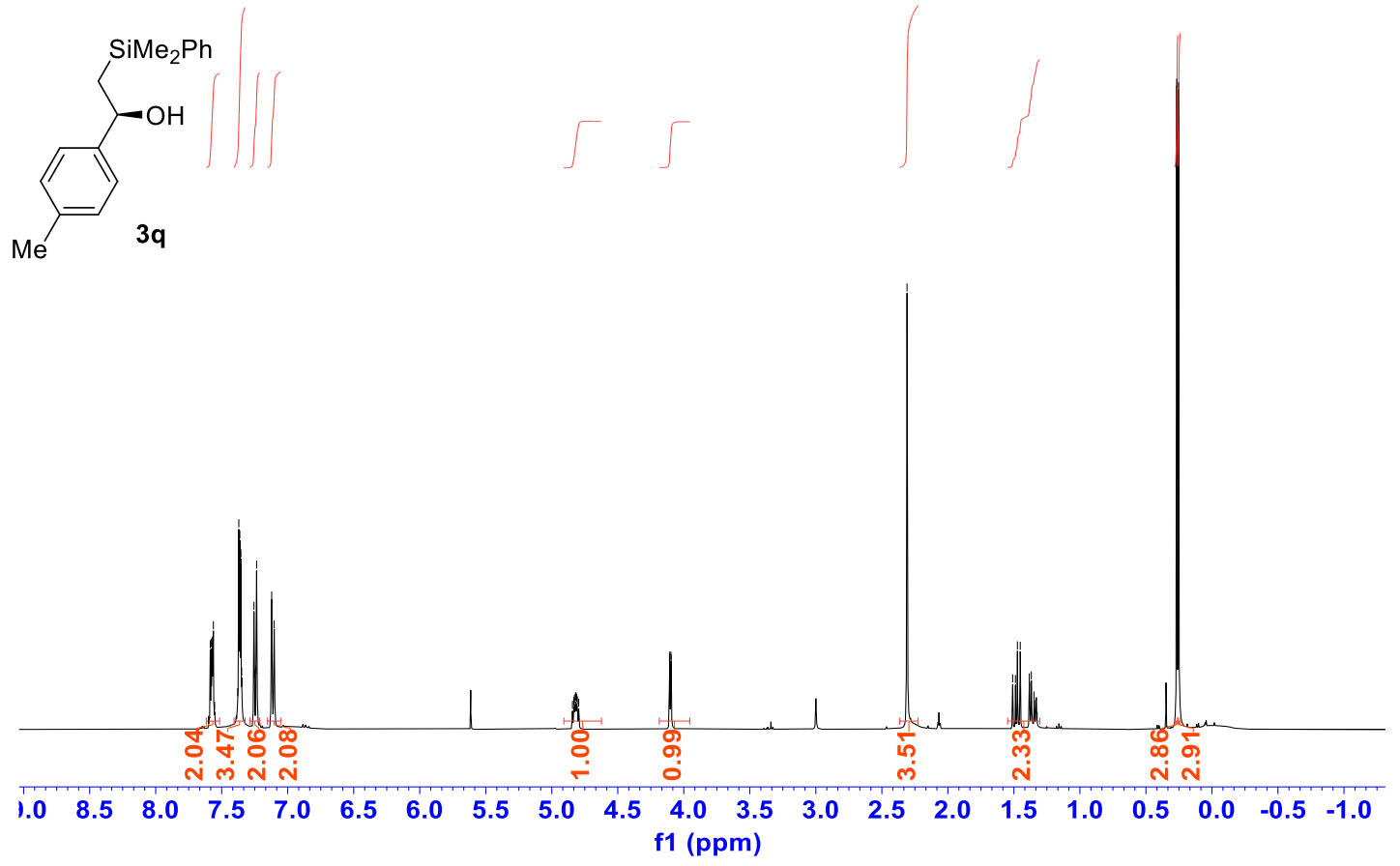

${ }^{13} \mathrm{C}\left\{{ }^{1} \mathrm{H}\right\}$ NMR of $\mathbf{3 q}(100 \mathrm{MHZ}$, Acetone)

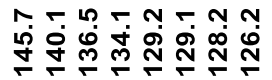

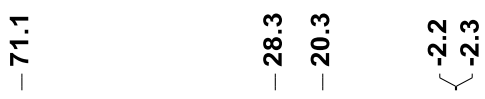

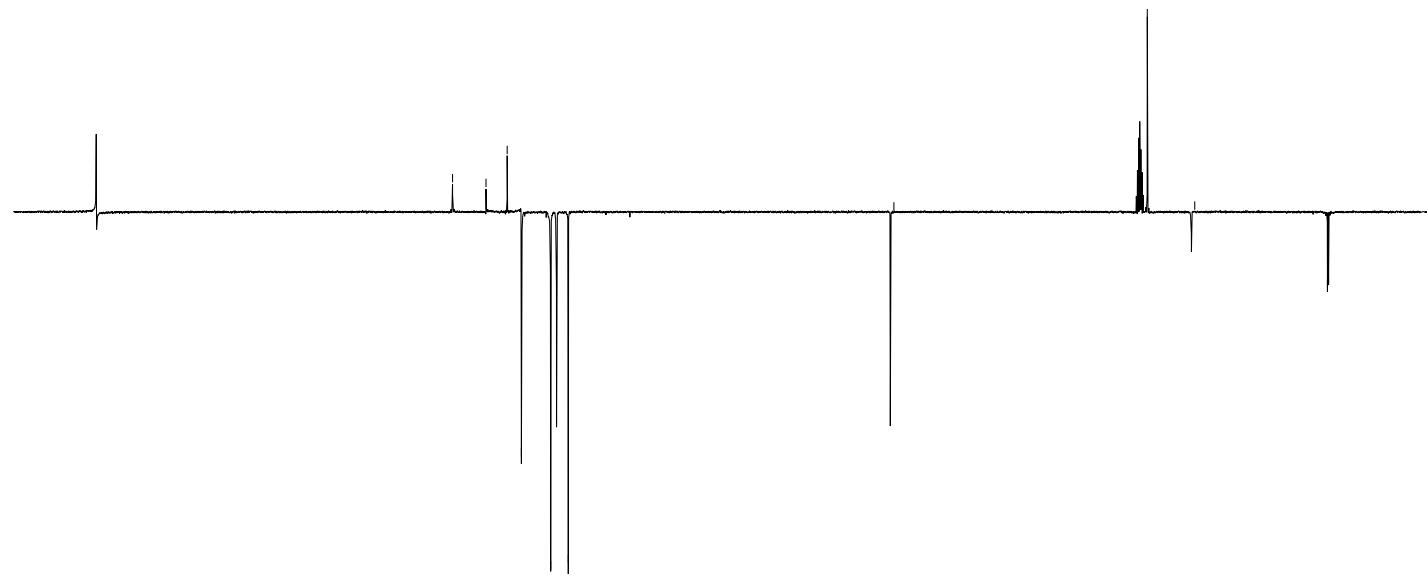

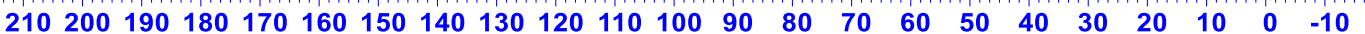
f1 (ppm) 
${ }^{1} \mathrm{H}$ NMR of 3r (400 MHZ, Acetone)

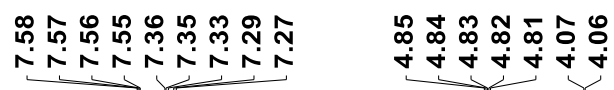

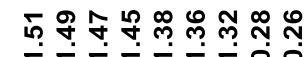

$\sum_{3 r}^{\mathrm{SiMe}_{2} \mathrm{Ph}}$
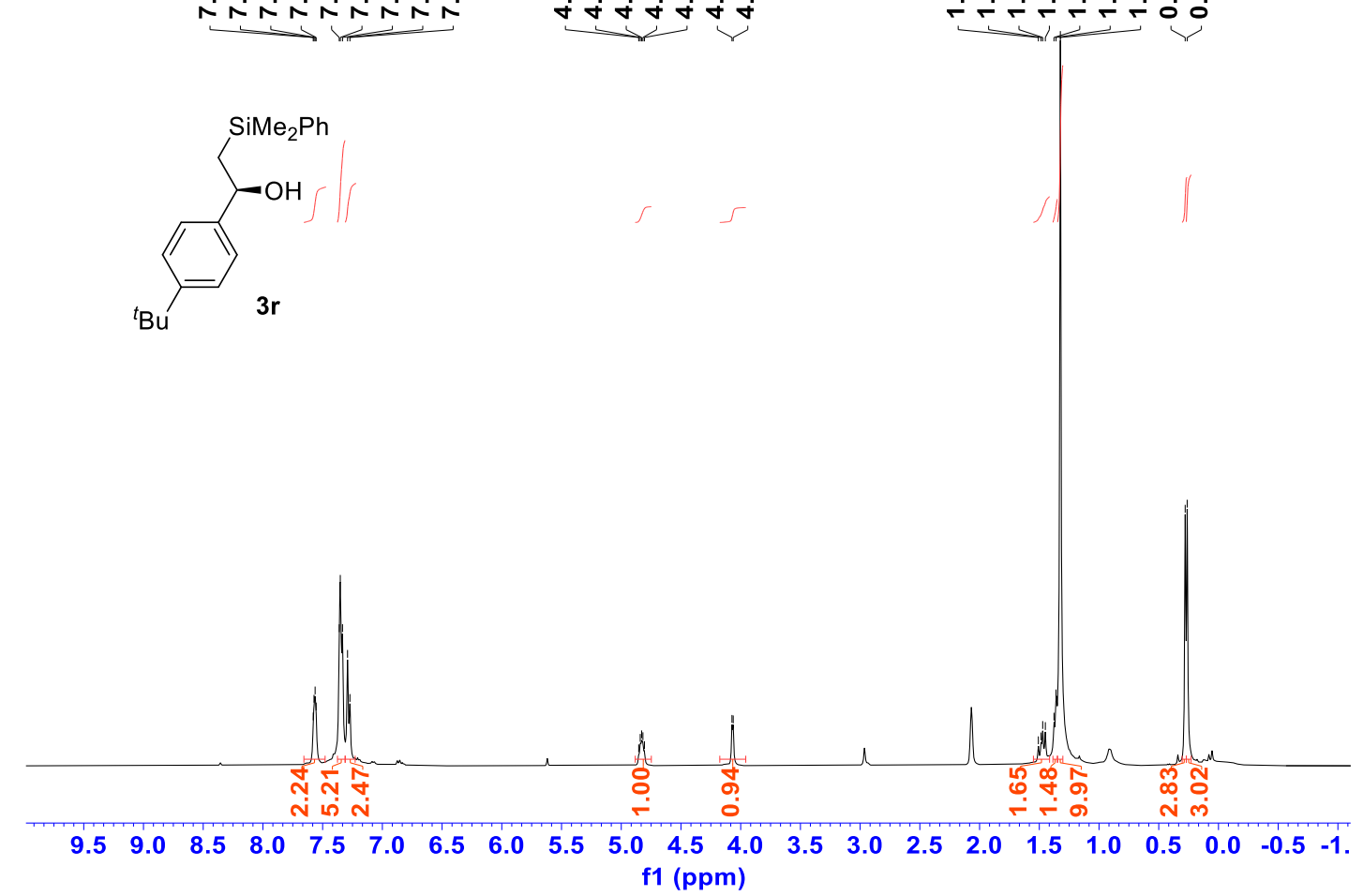

${ }^{13} \mathrm{C}\left\{{ }^{1} \mathrm{H}\right\}$ NMR of $3 \mathbf{r}$ (100 MHZ, Acetone)

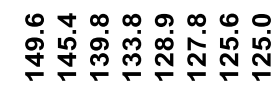

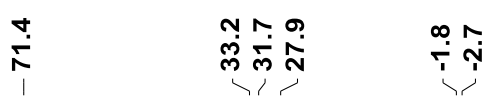

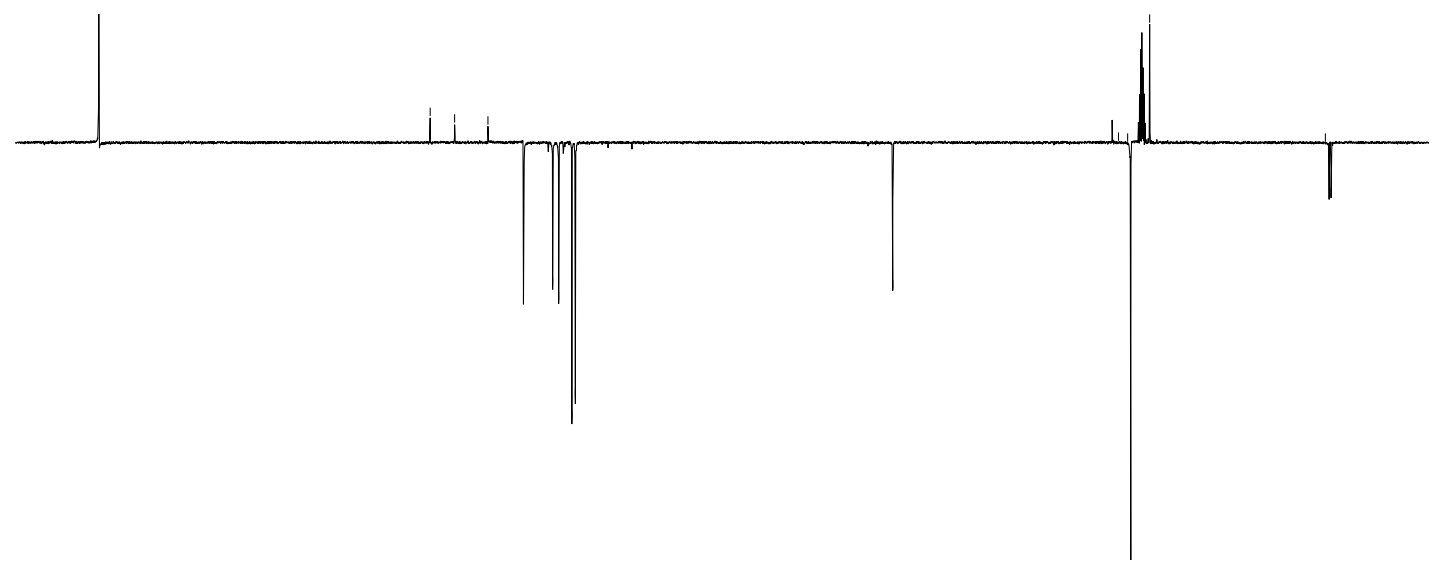

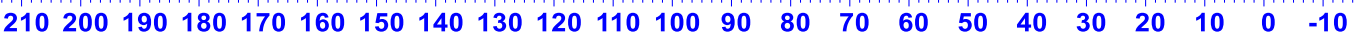
f1 (ppm) 
${ }^{1} \mathrm{H}$ NMR of 3s (400 MHZ, Acetone)

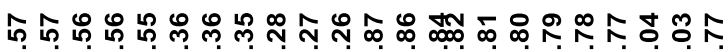

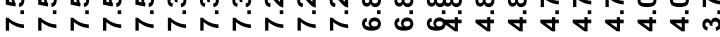

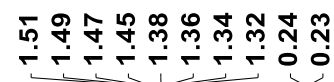

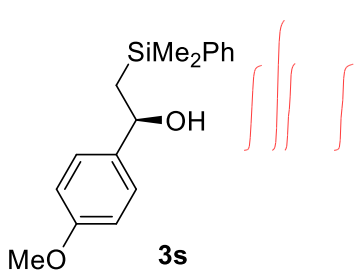

3s
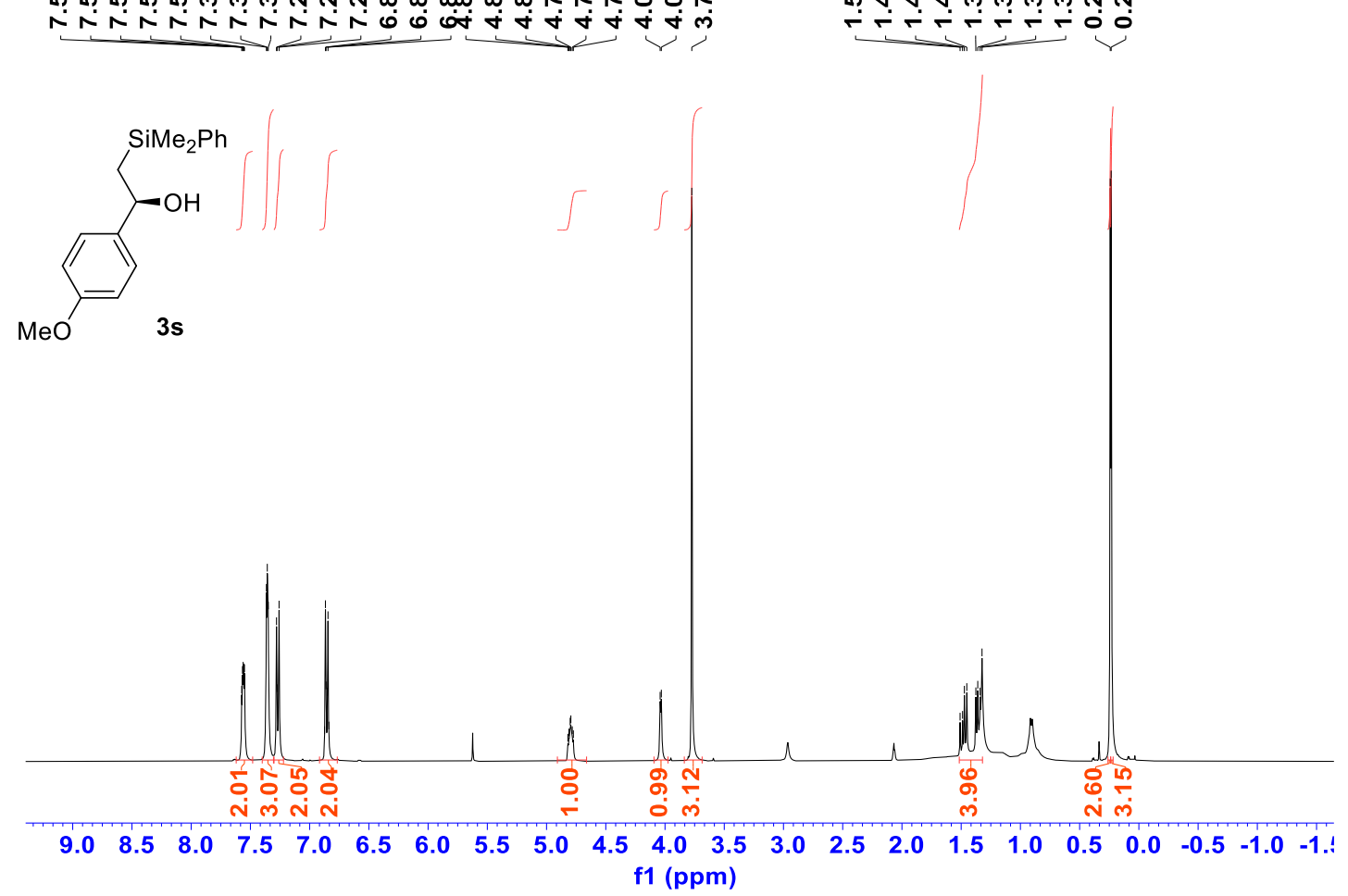

${ }^{13} \mathrm{C}\left\{{ }^{1} \mathrm{H}\right\}$ NMR of 3s (100 MHZ, Acetone)
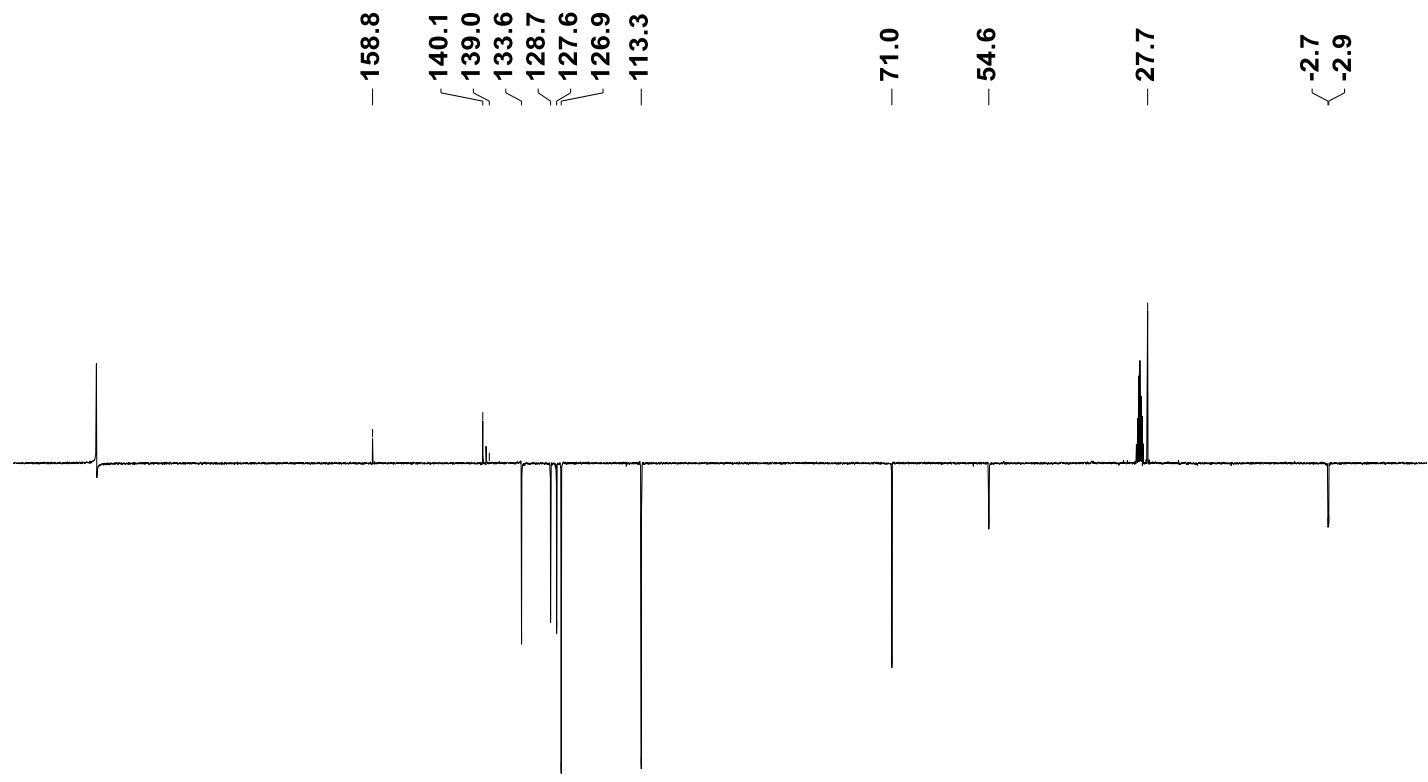

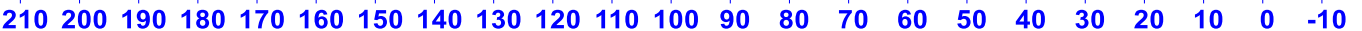
f1 (ppm) 
${ }^{1} \mathrm{H}$ NMR of $3 \mathrm{t}$ (400 MHZ, Acetone)

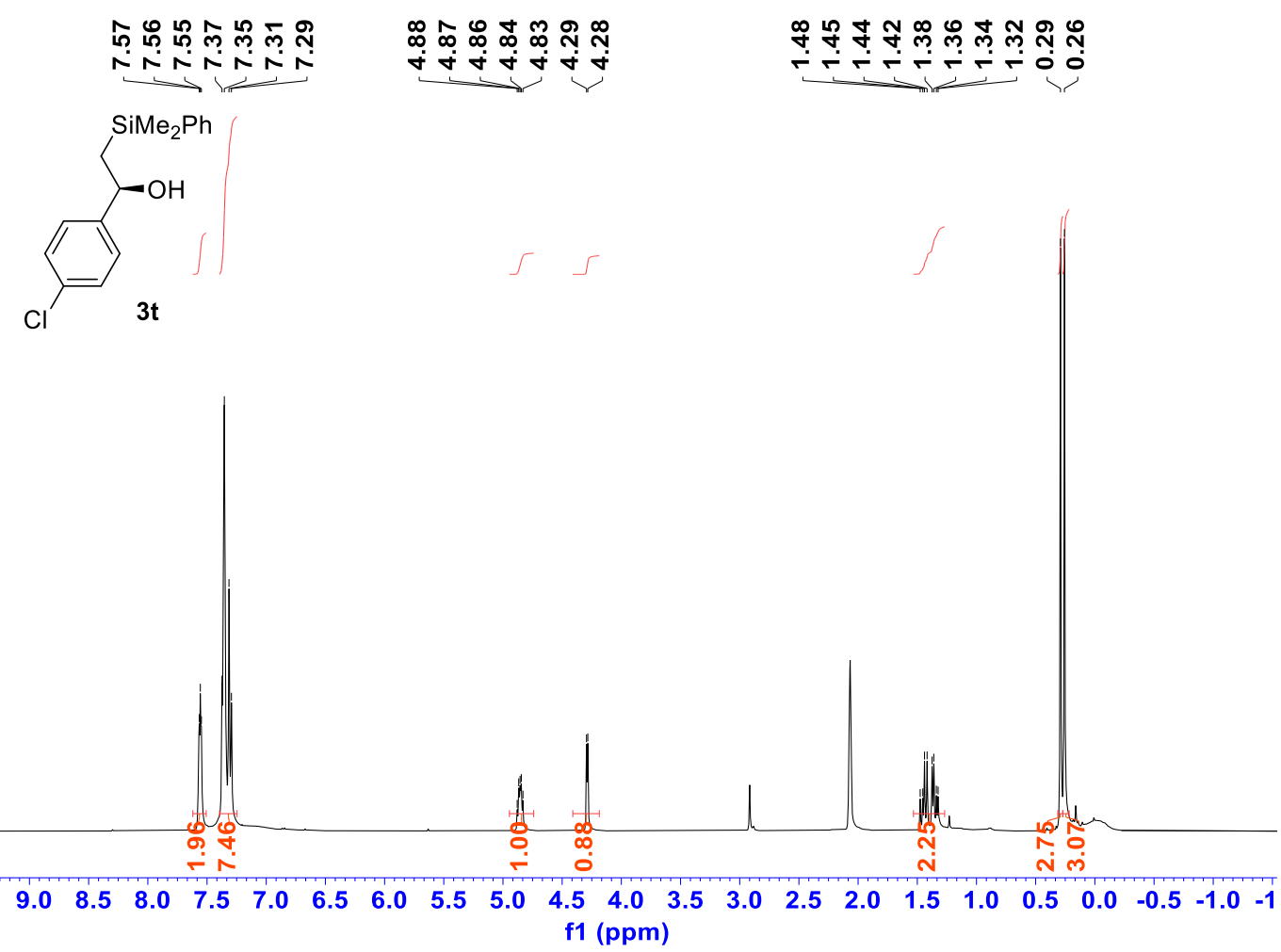

${ }^{13} \mathrm{C}\left\{{ }^{1} \mathrm{H}\right\}$ NMR of $3 \mathbf{t}(100 \mathrm{MHZ}$, Acetone)

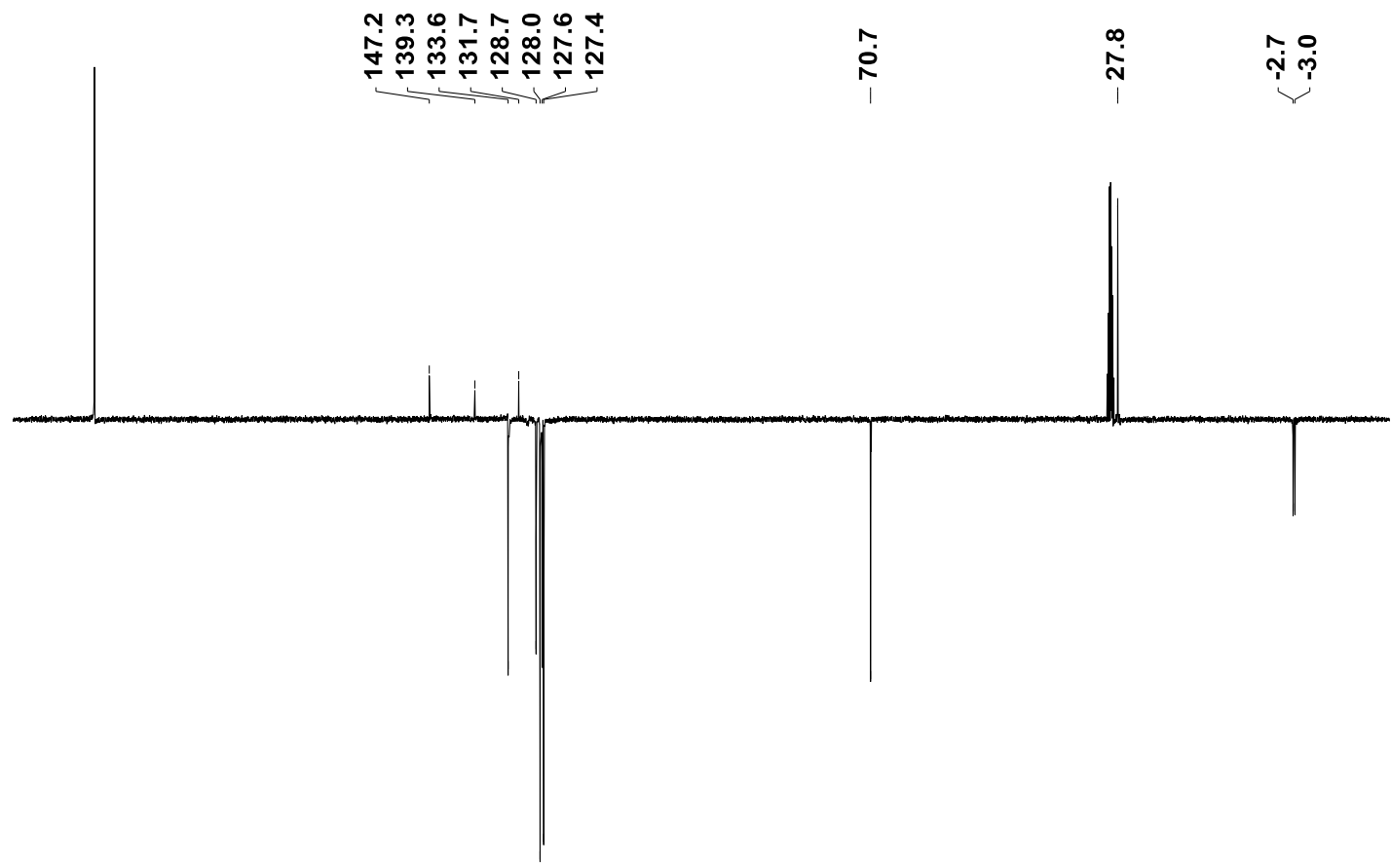

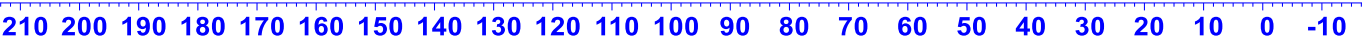
f1 (ppm) 
${ }^{1} \mathrm{H}$ NMR of 3u (400 MHZ, Acetone)

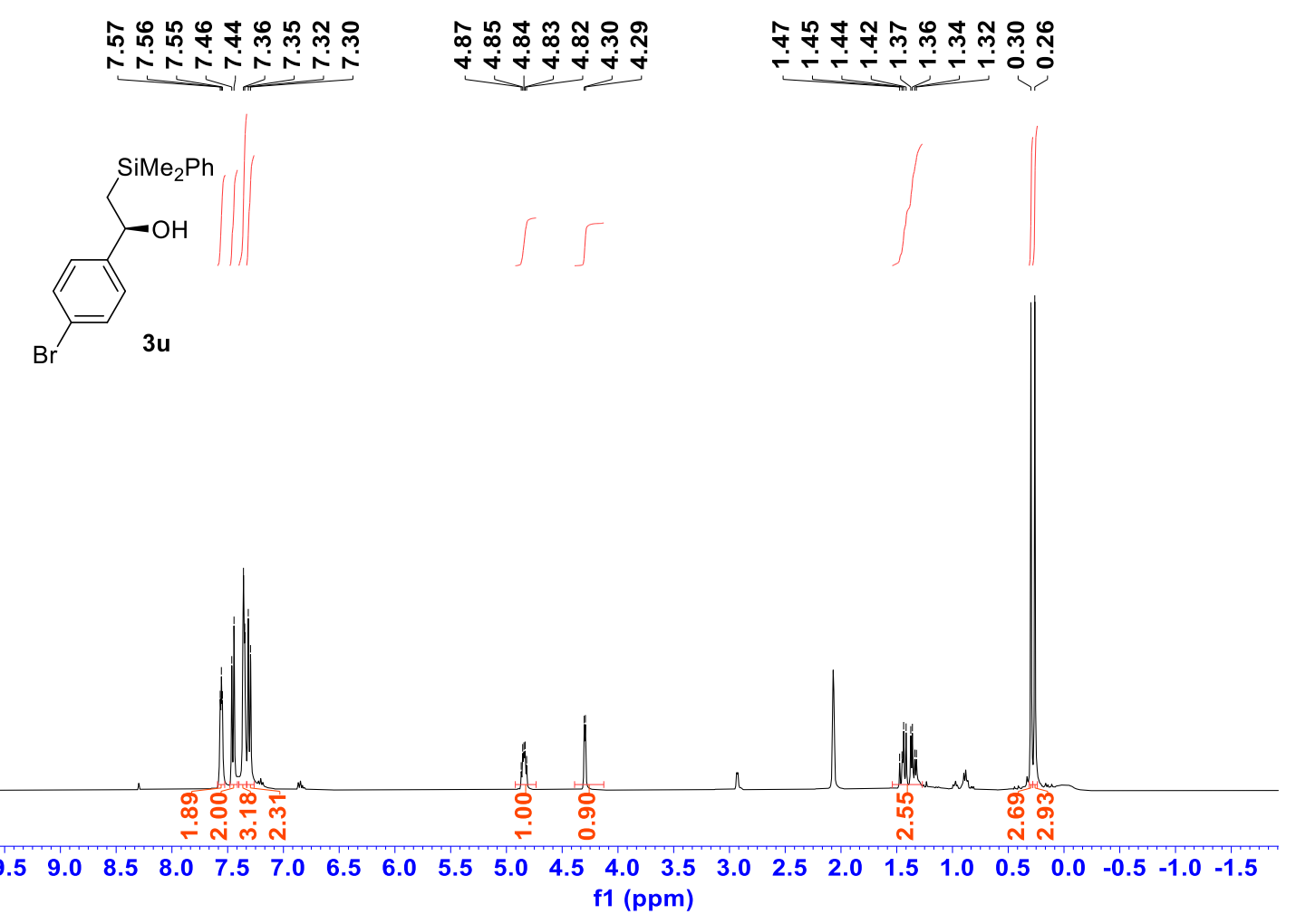

${ }^{13} \mathrm{C}\left\{{ }^{1} \mathrm{H}\right\}$ NMR of $3 \mathbf{u}(100 \mathrm{MHZ}$, Acetone)

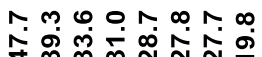

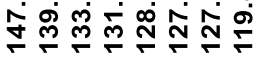

$\hat{i} \quad \stackrel{\hat{N}}{i} \quad \stackrel{0}{i}$

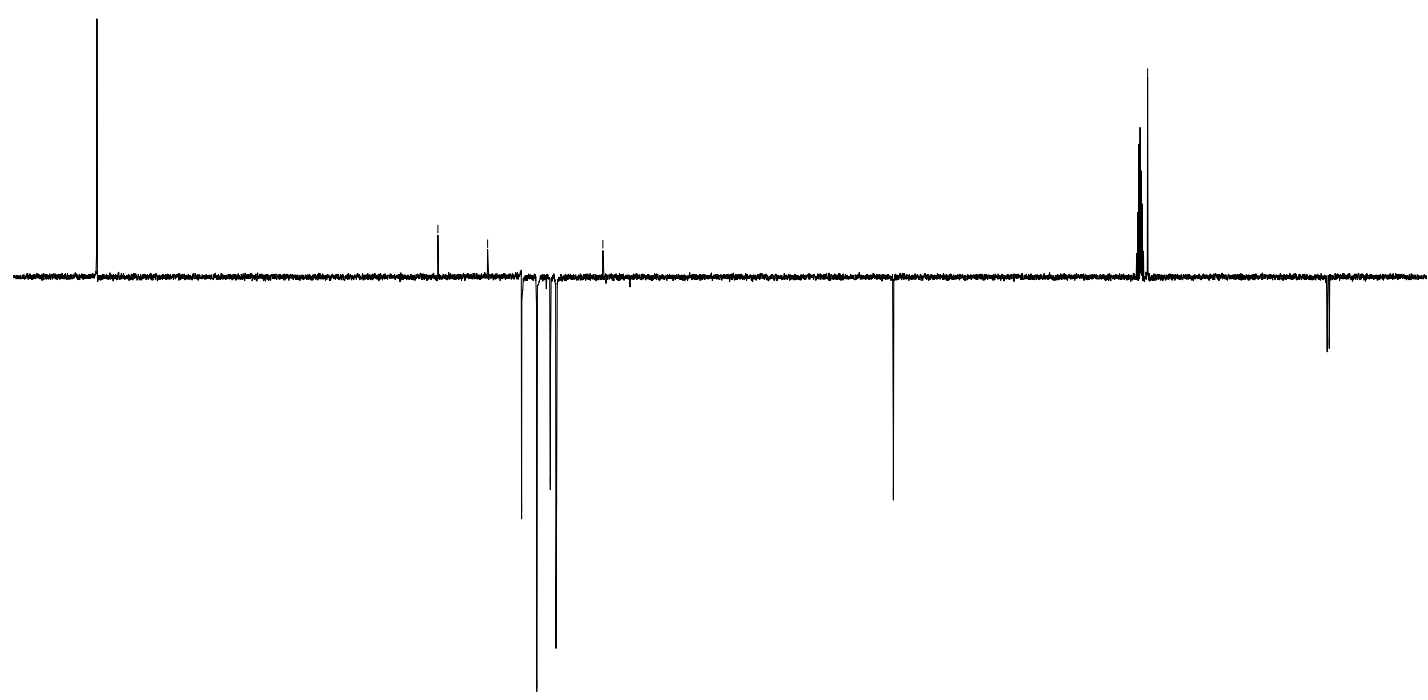

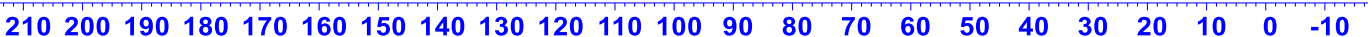
f1 (ppm) 
${ }^{1} \mathrm{H}$ NMR of $3 \mathbf{v}$ (400 MHZ, Acetone)

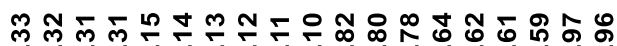

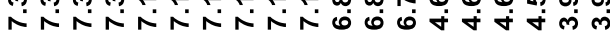

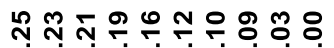

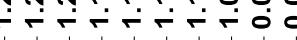
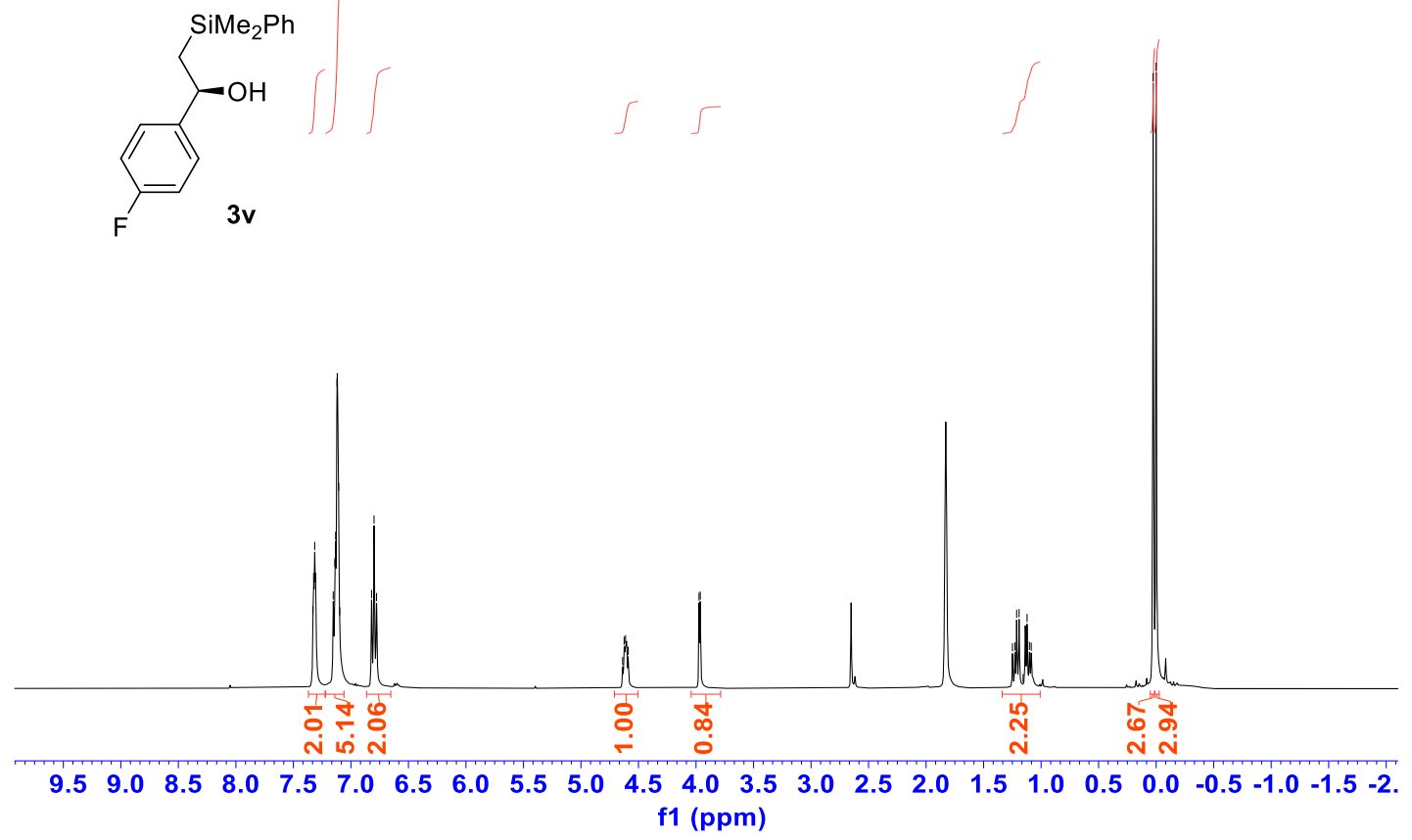

${ }^{13} \mathrm{C}\left\{{ }^{1} \mathrm{H}\right\}$ NMR of 3v (100 MHZ, Acetone)

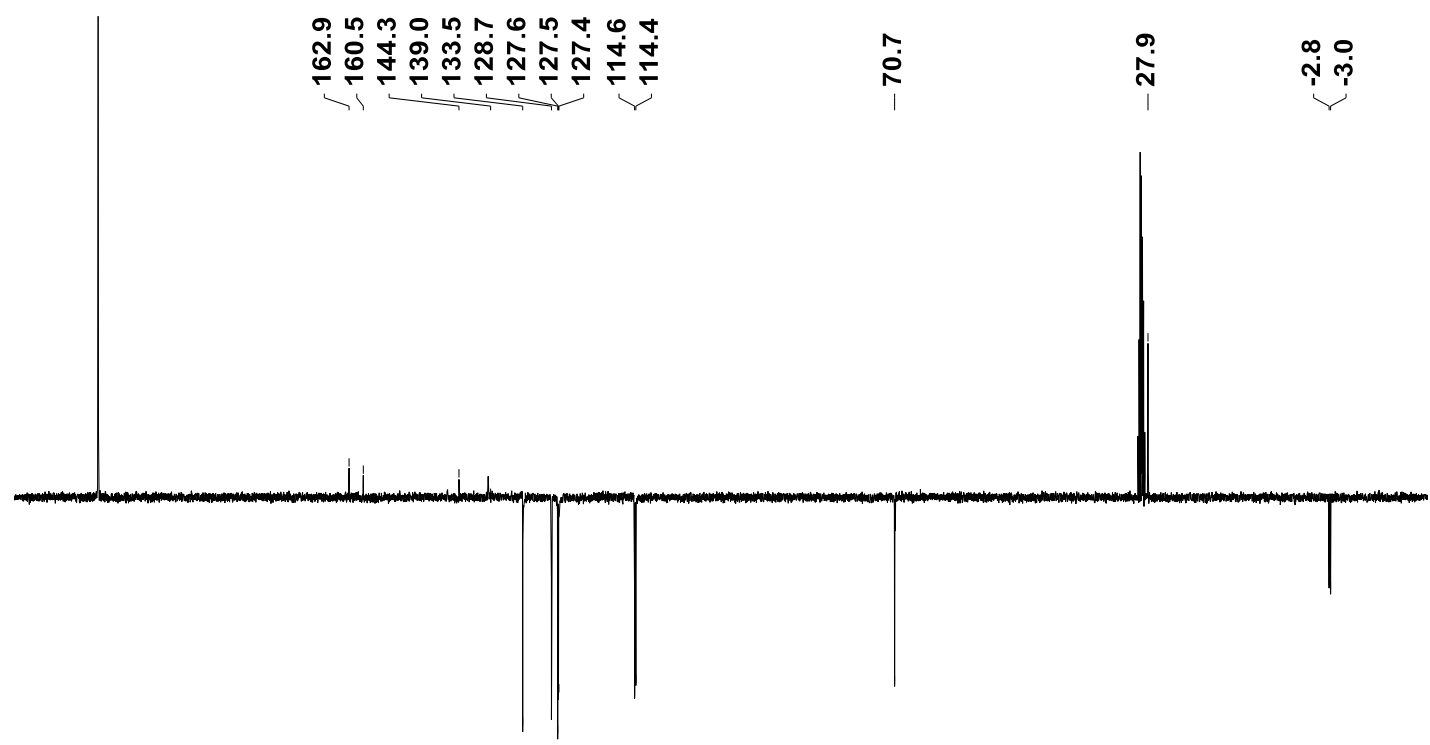


${ }^{19} \mathrm{~F}$ NMR of 3v (377 $\left.\mathrm{MHZ}, \mathrm{CDCl}_{3}\right)$

$\frac{\bar{s}}{\bar{i}}$

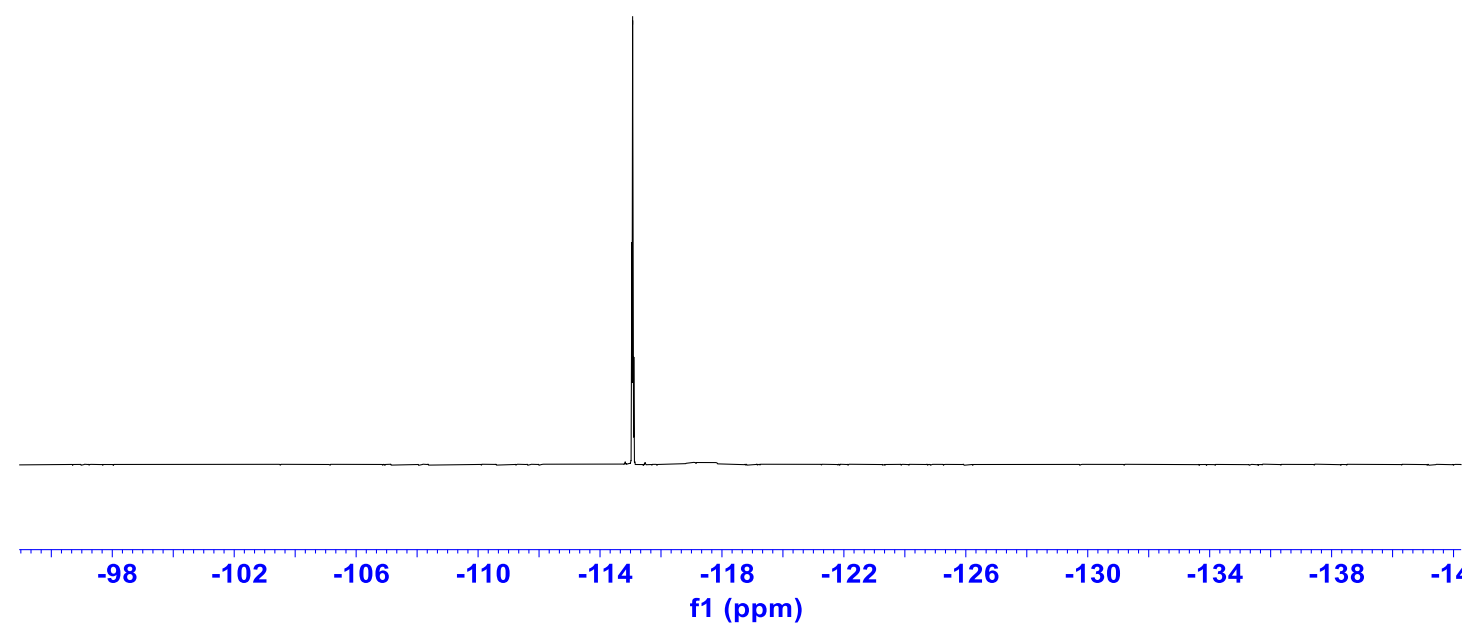


${ }^{1} \mathrm{H}$ NMR of 3w (400 MHZ, Acetone)

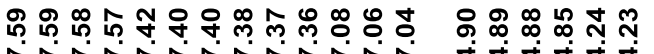

N N N N N N N N

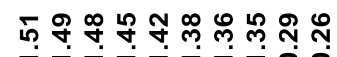<smiles>OC(COc1ccccc1)c1ccc(C(F)(F)F)cc1</smiles>

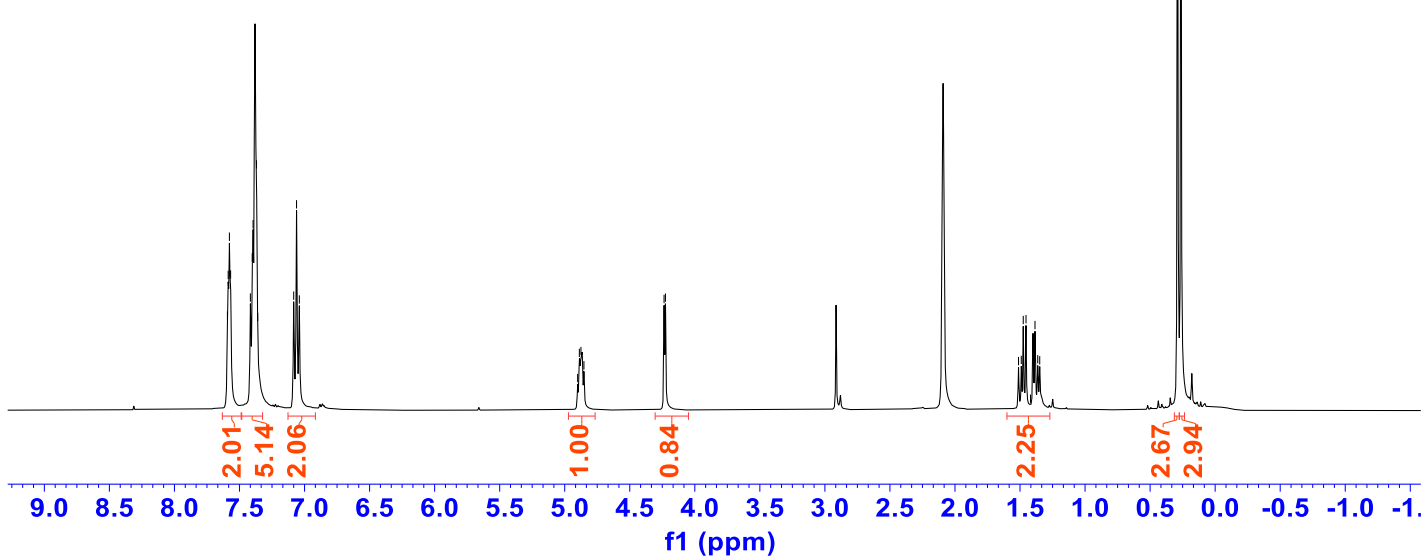

${ }^{13} \mathrm{C}\left\{{ }^{1} \mathrm{H}\right\}$ NMR of 3w (100 MHZ, Acetone)
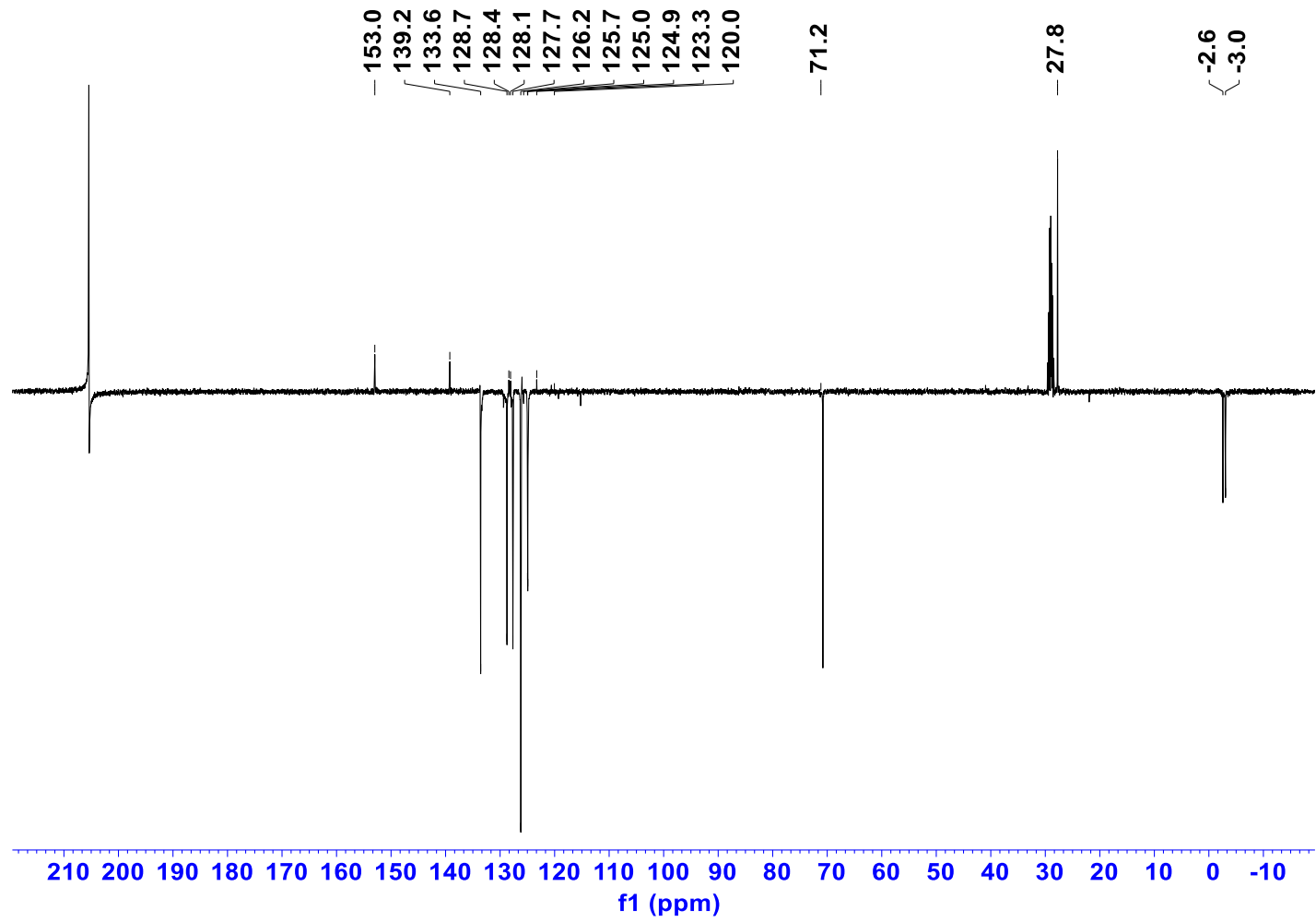
${ }^{19} \mathrm{~F}$ NMR of 3w (377 MHZ, $\left.\mathrm{CDCl}_{3}\right)$

$$
\text { ஸ̣ }
$$

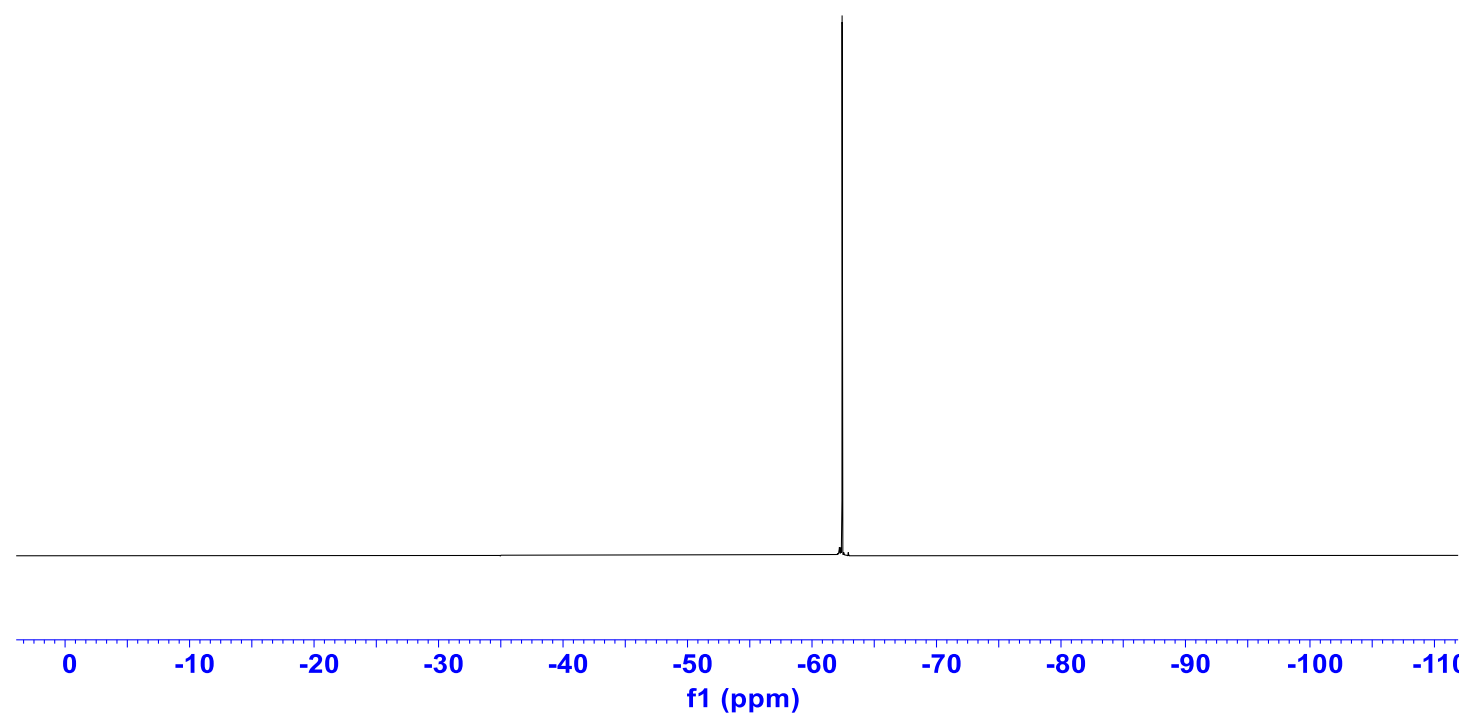


${ }^{1} \mathrm{H}$ NMR of $4 \mathbf{a}\left(400 \mathrm{MHZ}, \mathrm{CDCl}_{3}\right)$

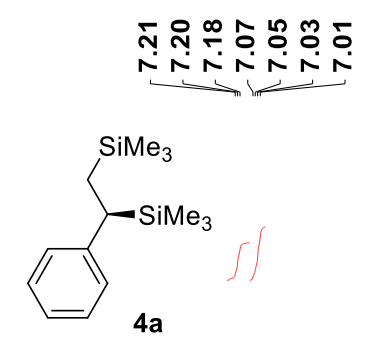

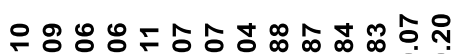

赵

ก

$4 a$

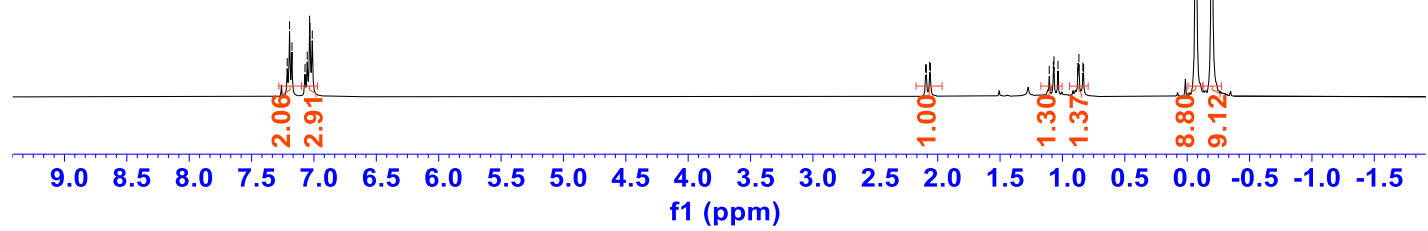

${ }^{13} \mathrm{C}\left\{{ }^{1} \mathrm{H}\right\}$ NMR of $\mathbf{4 a}\left(100 \mathrm{MHZ}, \mathrm{CDCl}_{3}\right)$

草 过

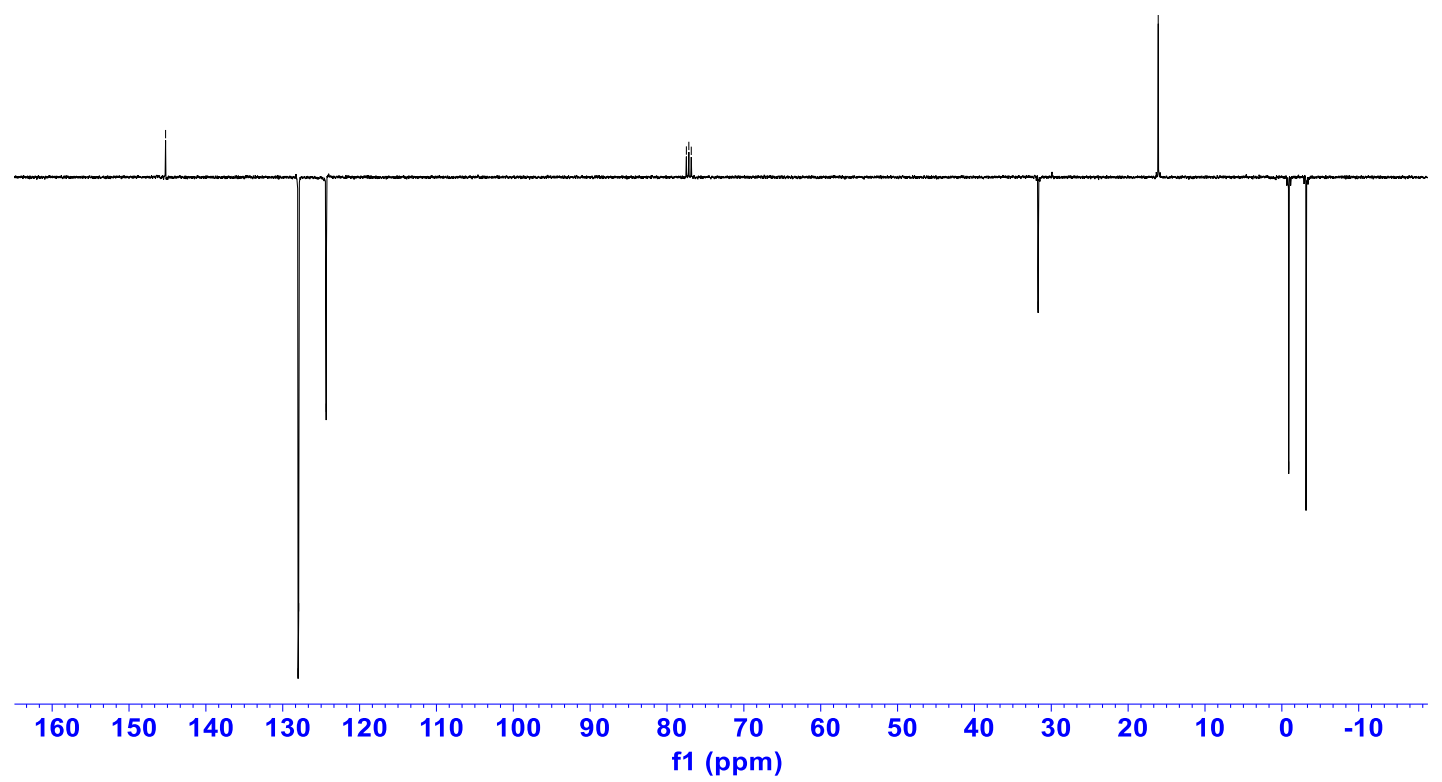


${ }^{1} \mathrm{H}$ NMR of $4 \mathbf{b}\left(400 \mathrm{MHZ}, \mathrm{CDCl}_{3}\right)$

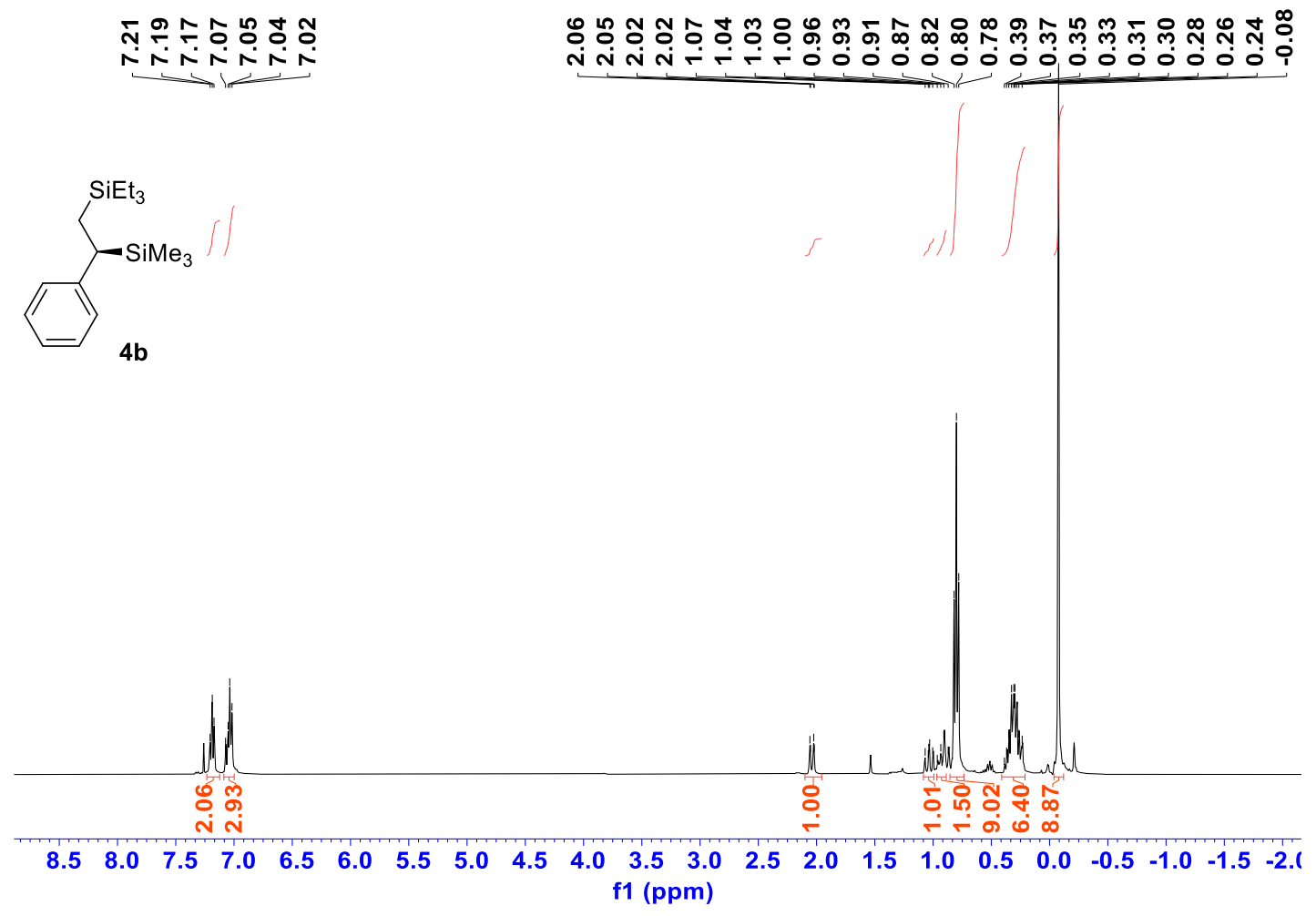

${ }^{13} \mathrm{C}\left\{{ }^{1} \mathrm{H}\right\}$ NMR of $\mathbf{4 b}\left(100 \mathrm{MHZ}, \mathrm{CDCl}_{3}\right)$

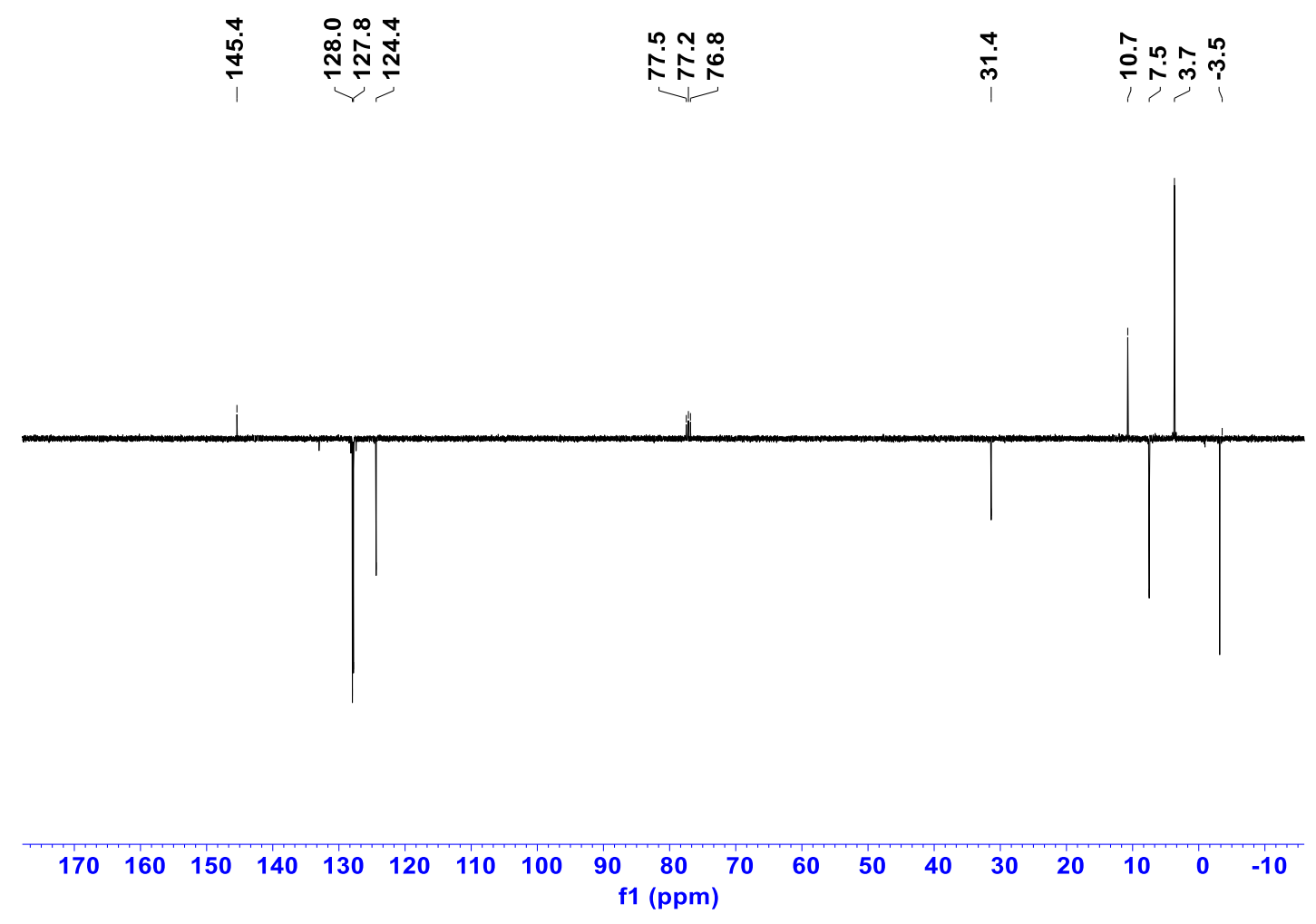


${ }^{1} \mathrm{H}$ NMR of $4 \mathbf{c}\left(400 \mathrm{MHZ}, \mathrm{CDCl}_{3}\right)$

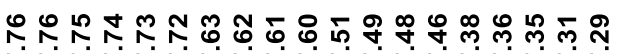

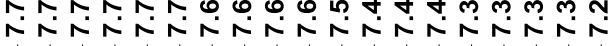

รุ

nhtr-trogo<smiles>CC(CC(C)c1ccccc1)c1ccccc1</smiles>

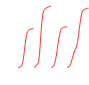

$4 c$
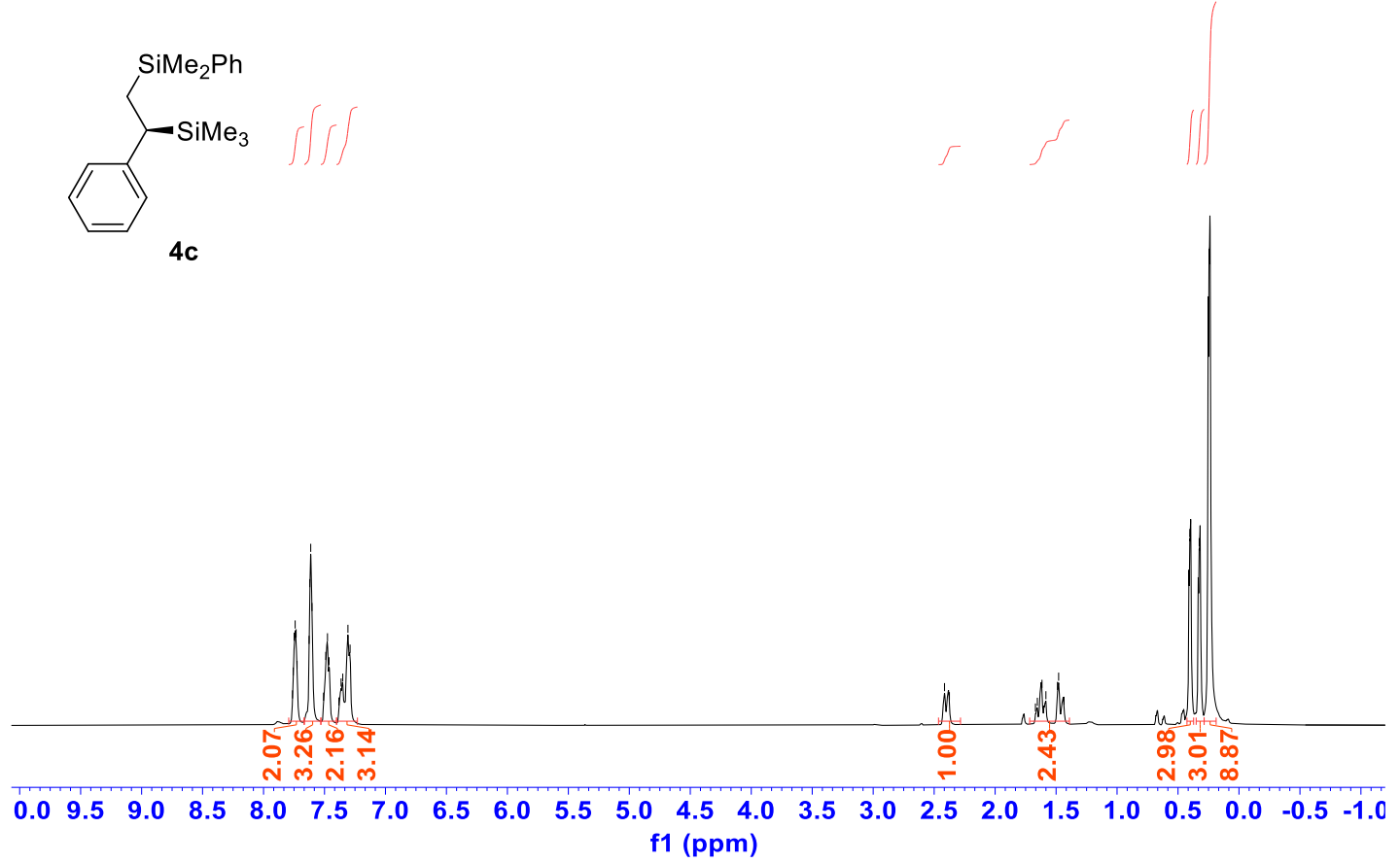

${ }^{13} \mathrm{C}\left\{{ }^{1} \mathrm{H}\right\}$ NMR of $4 \mathbf{c}\left(100 \mathrm{MHZ}, \mathrm{CDCl}_{3}\right)$

$\infty \infty \omega \infty$

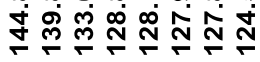

مึ

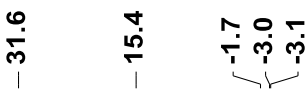

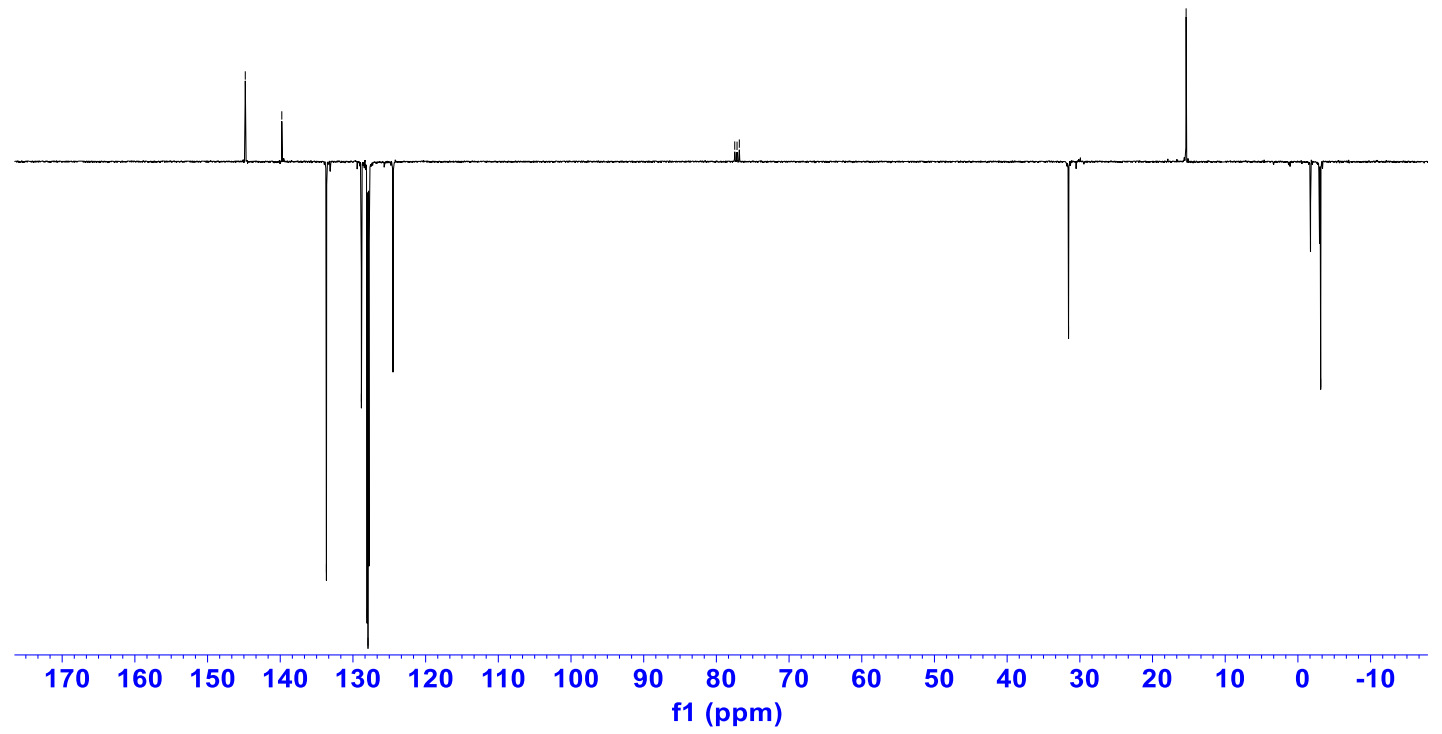


${ }^{1} \mathrm{H}$ NMR of $4 \mathbf{d}\left(400 \mathrm{MHZ}, \mathrm{CDCl}_{3}\right)$

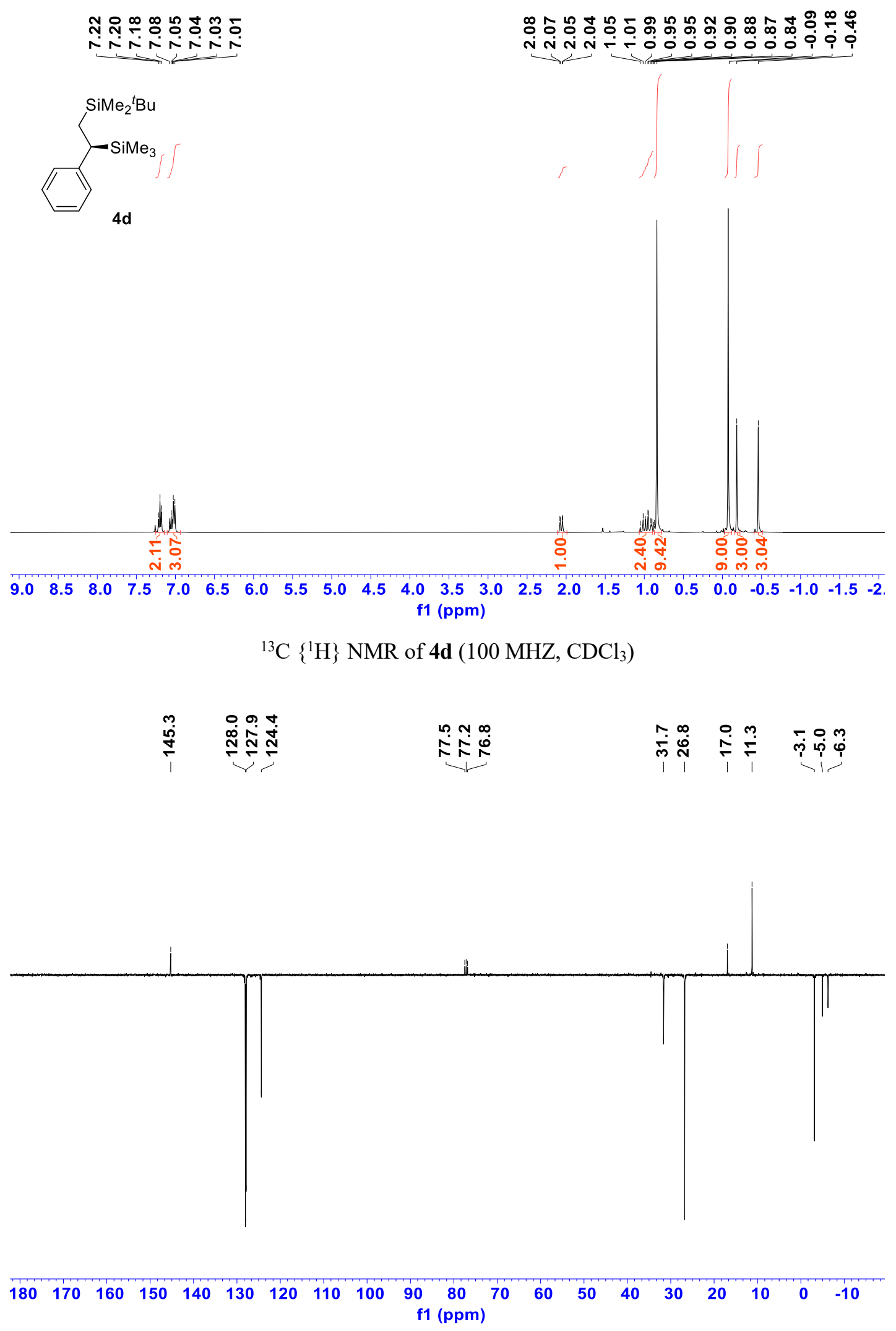

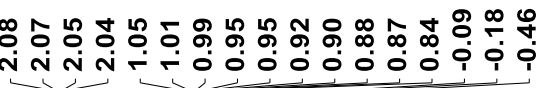

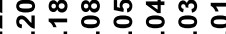


${ }^{1} \mathrm{H}$ NMR of $4 \mathbf{e}\left(400 \mathrm{MHZ}, \mathrm{CDCl}_{3}\right)$

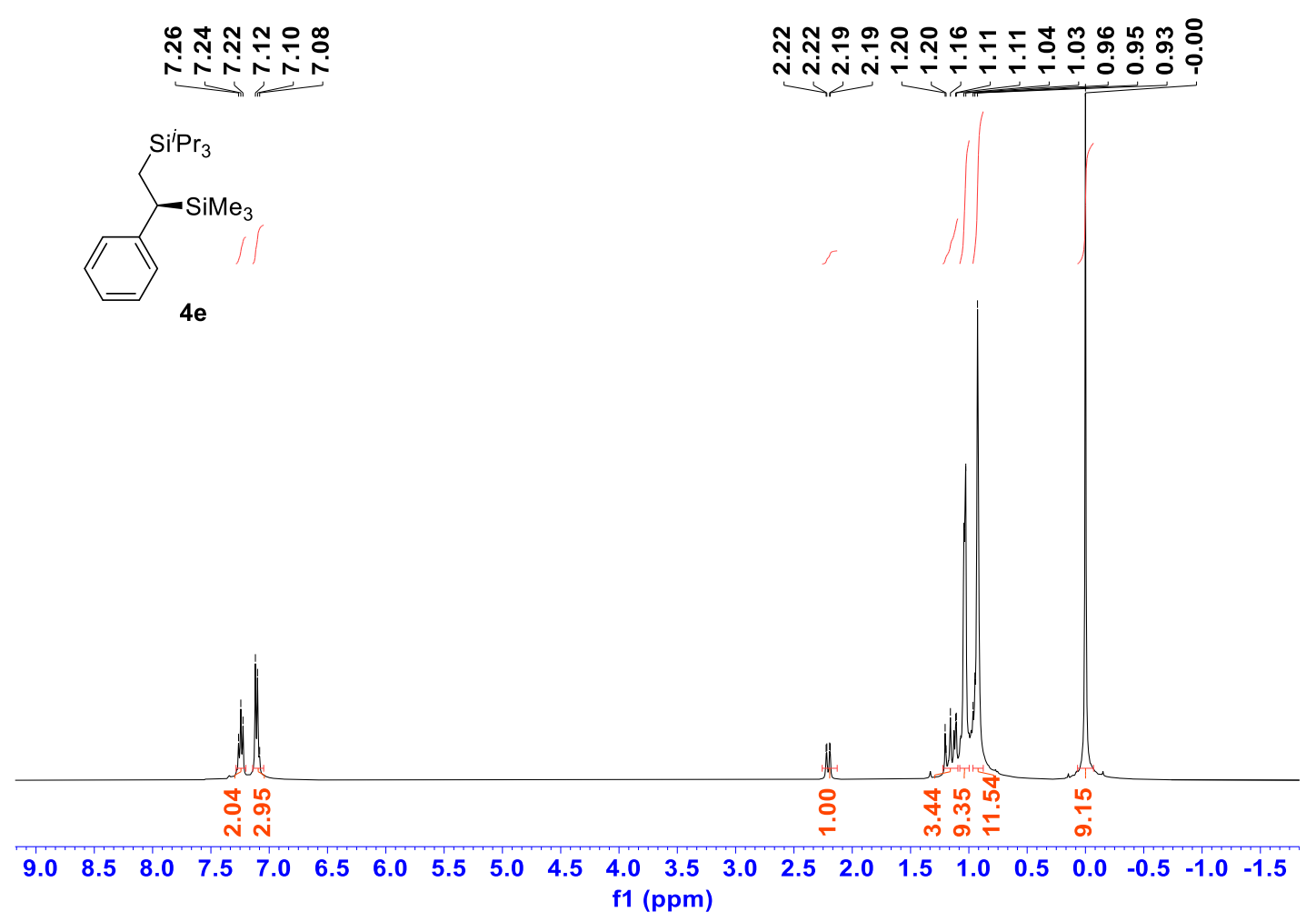

${ }^{13} \mathrm{C}\left\{{ }^{1} \mathrm{H}\right\}$ NMR of $4 \mathbf{e}\left(100 \mathrm{MHZ}, \mathrm{CDCl}_{3}\right)$

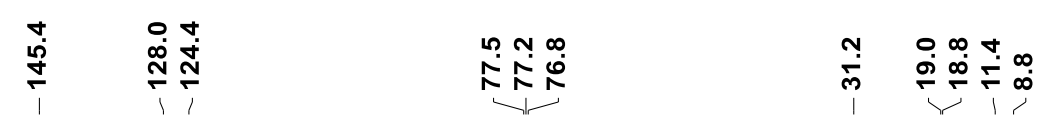

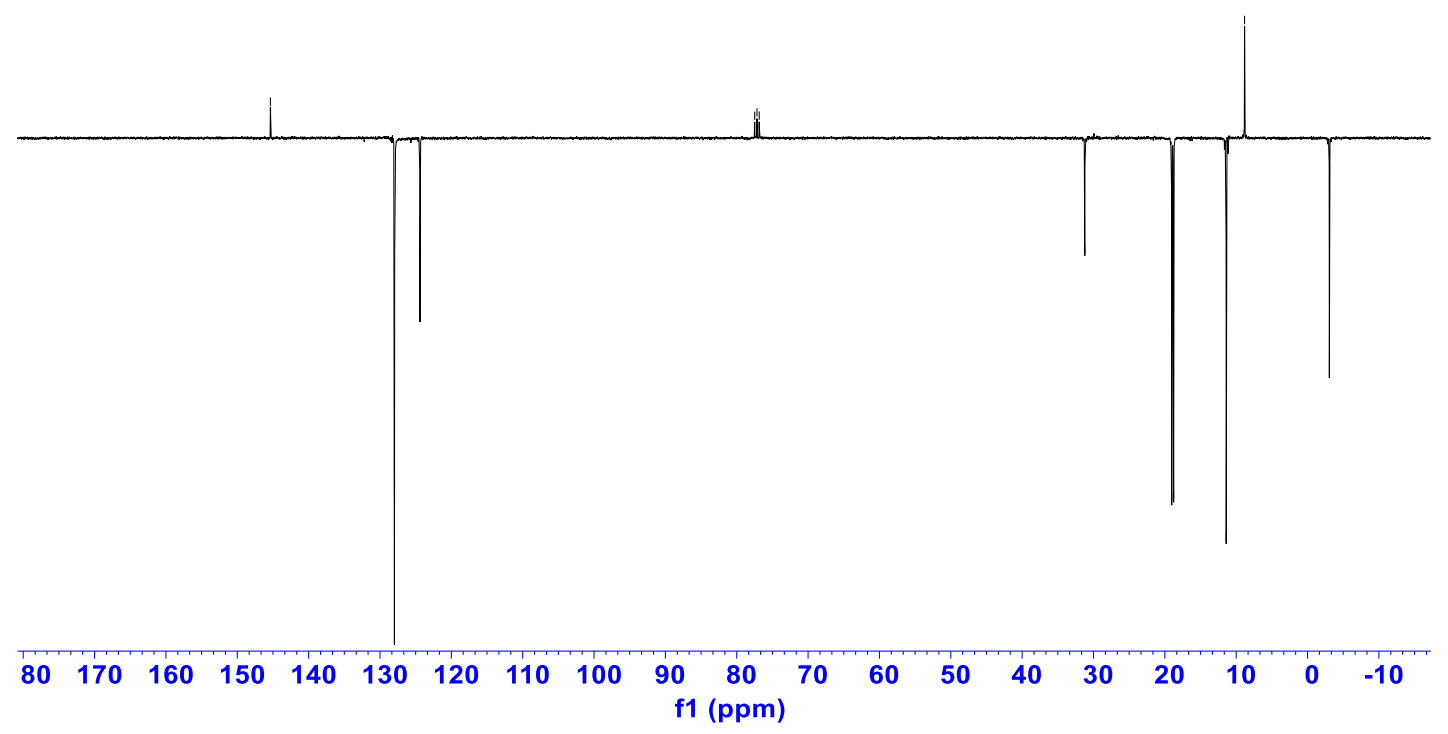


${ }^{1} \mathrm{H}$ NMR of $\mathbf{4 f}(400 \mathrm{MHZ}$, Acetone)

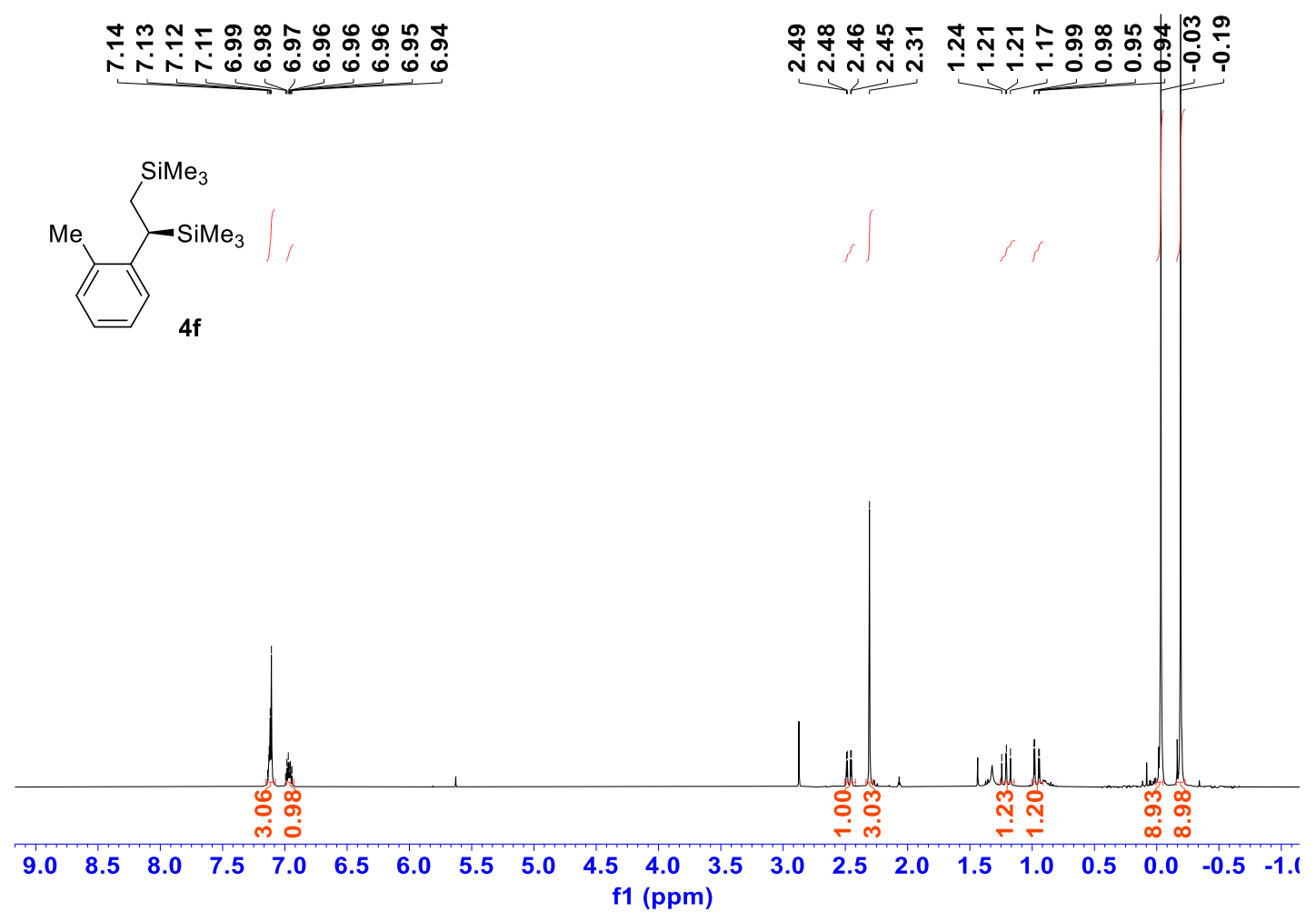

${ }^{13} \mathrm{C}\left\{{ }^{1} \mathrm{H}\right\}$ NMR of $\mathbf{4 f}(100 \mathrm{MHZ}$, Acetone)
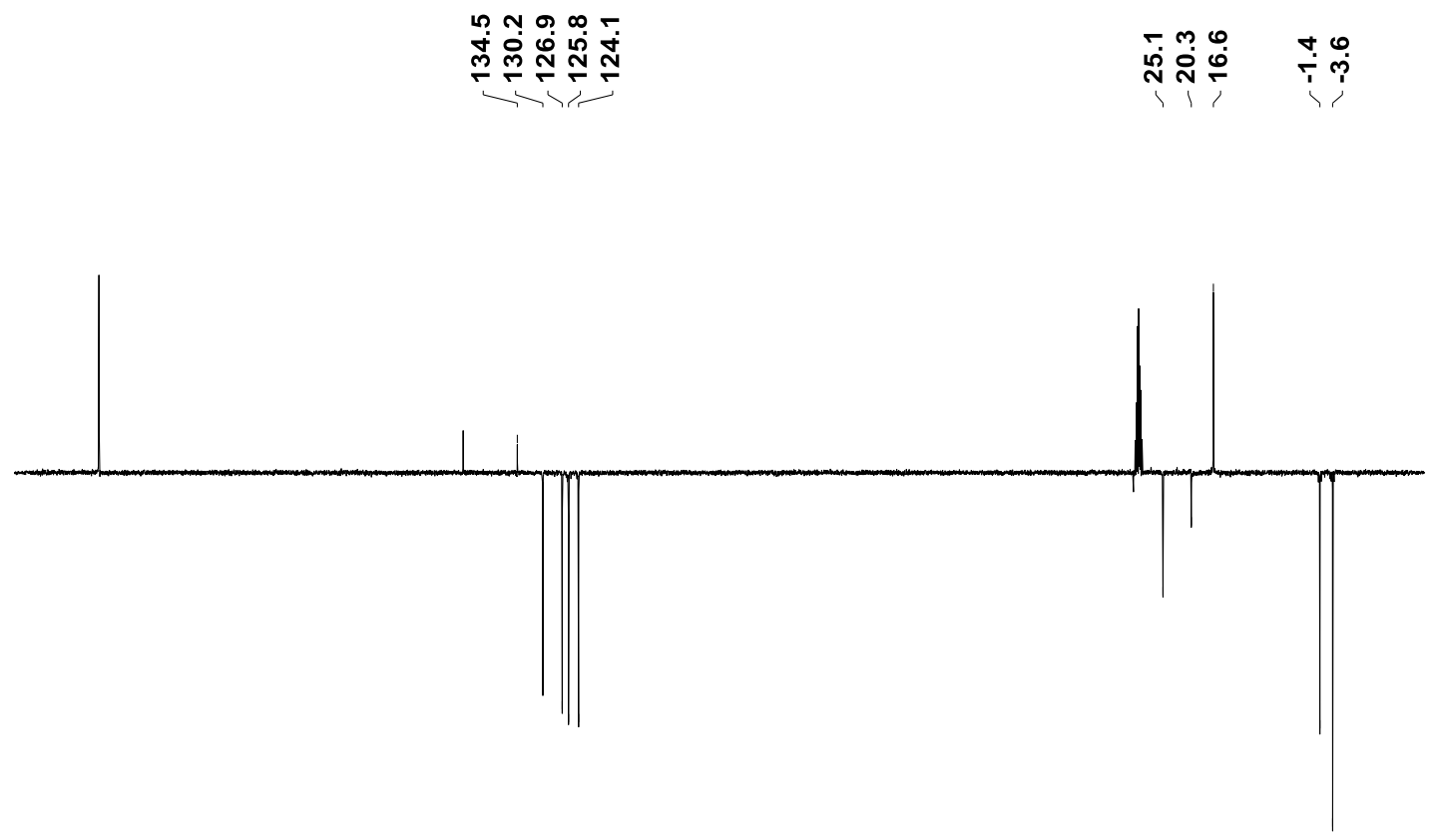

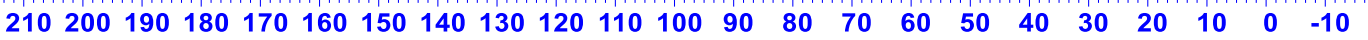
f1 (ppm) 
${ }^{1} \mathrm{H}$ NMR of $4 \mathrm{~g}$ (400 MHZ, Acetone)

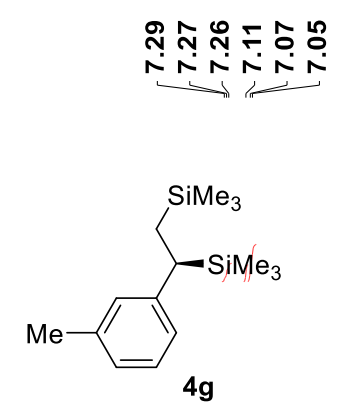

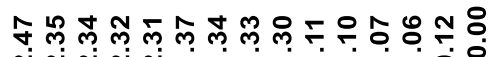

ininn

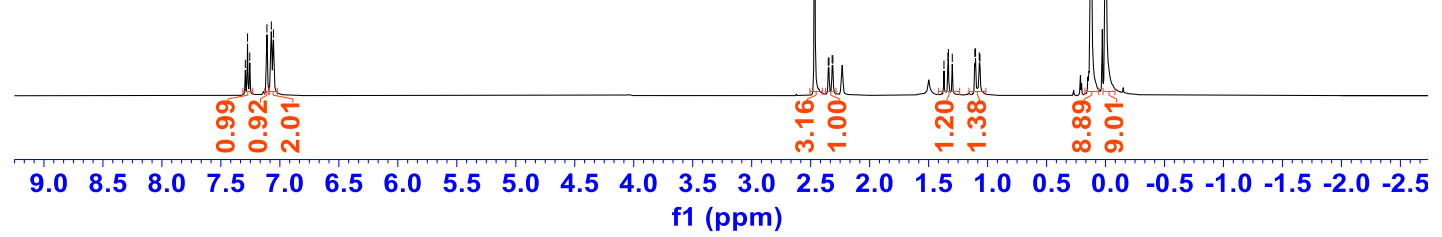

${ }^{13} \mathrm{C}\left\{{ }^{1} \mathrm{H}\right\}$ NMR of $4 \mathbf{g}$ (100 MHZ, Acetone)
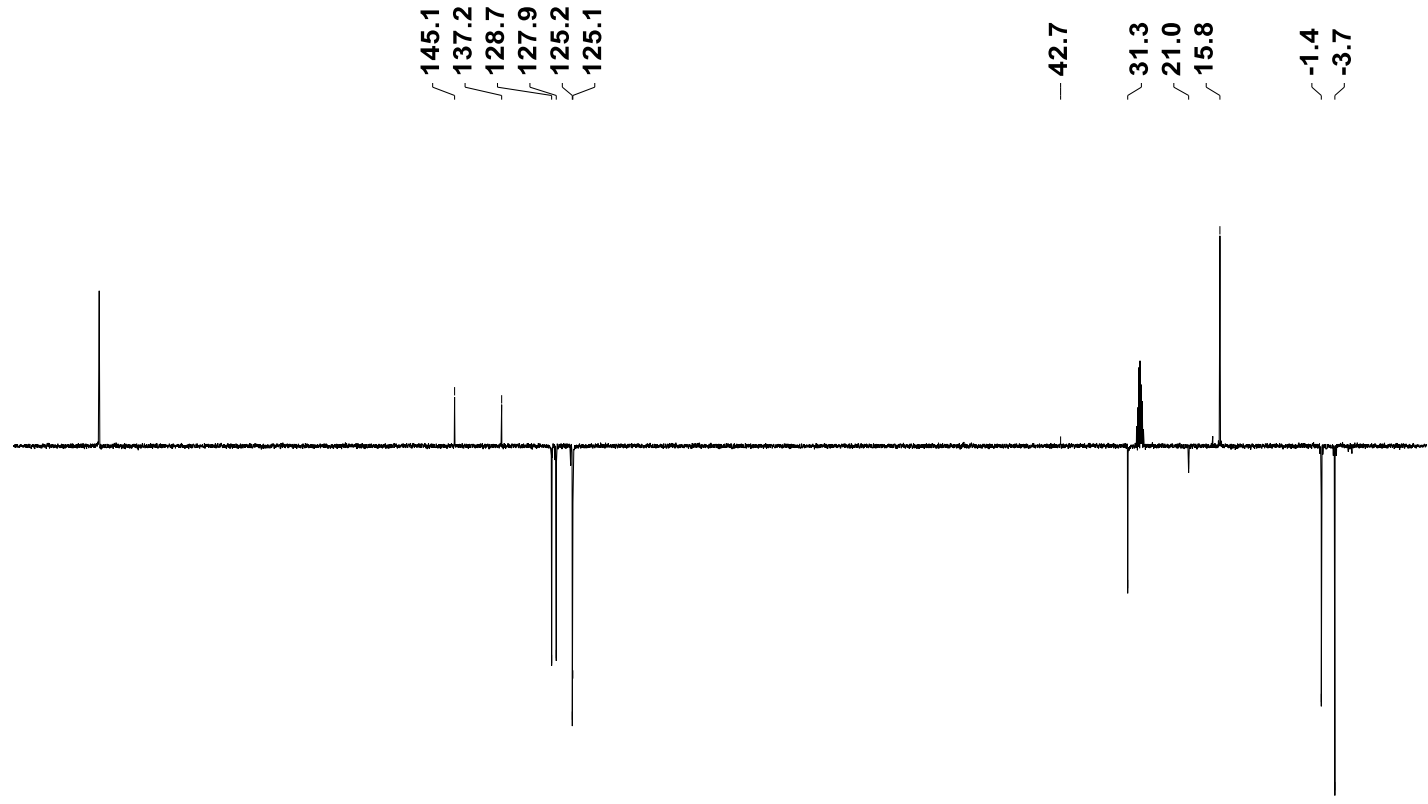

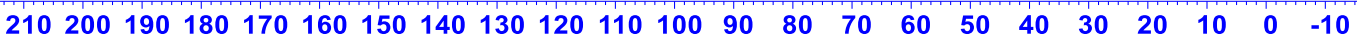
f1 (ppm) 
${ }^{1} \mathrm{H}$ NMR of $\mathbf{4 h}$ (400 MHZ, Acetone)

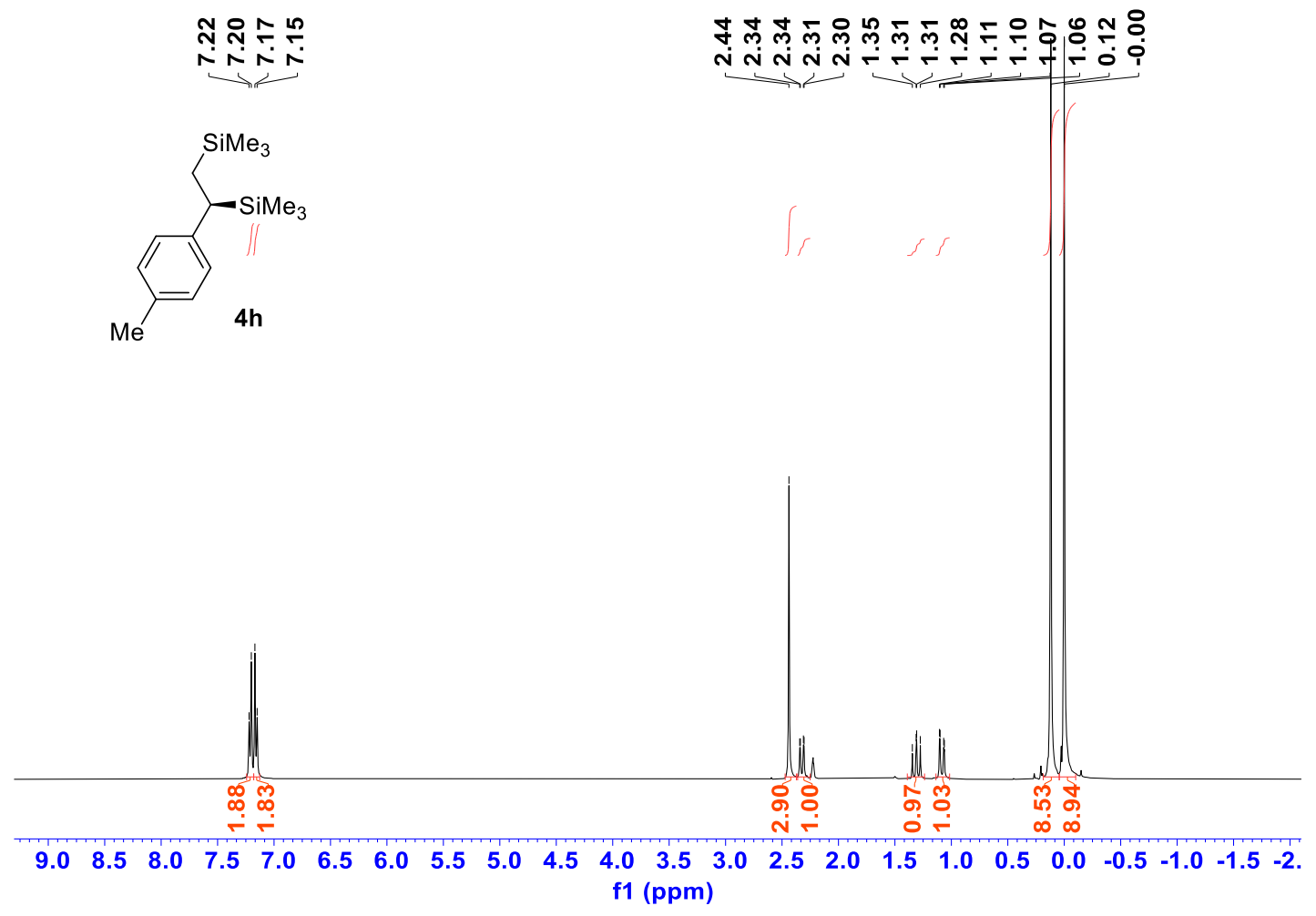

${ }^{13} \mathrm{C}\left\{{ }^{1} \mathrm{H}\right\}$ NMR of $\mathbf{4 h}(100 \mathrm{MHZ}$, Acetone)

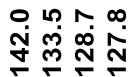

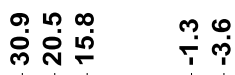

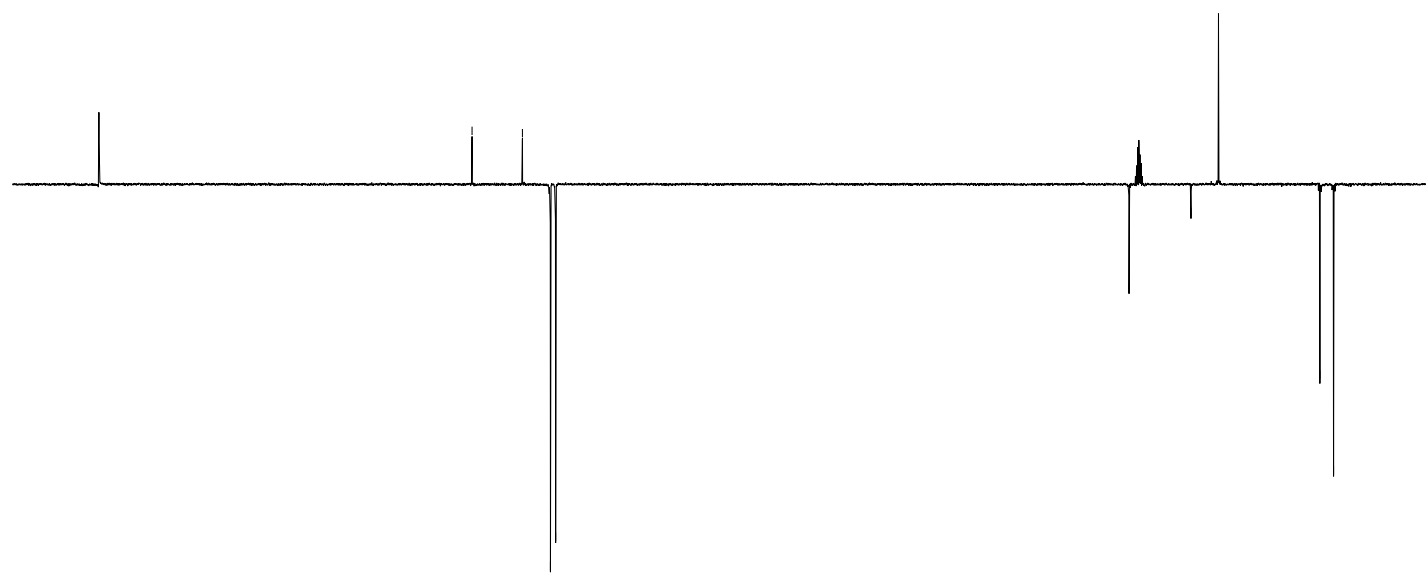

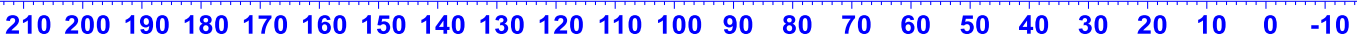
f1 (ppm) 
${ }^{1} \mathrm{H}$ NMR of $4 \mathbf{i}$ (400 MHZ, Acetone)

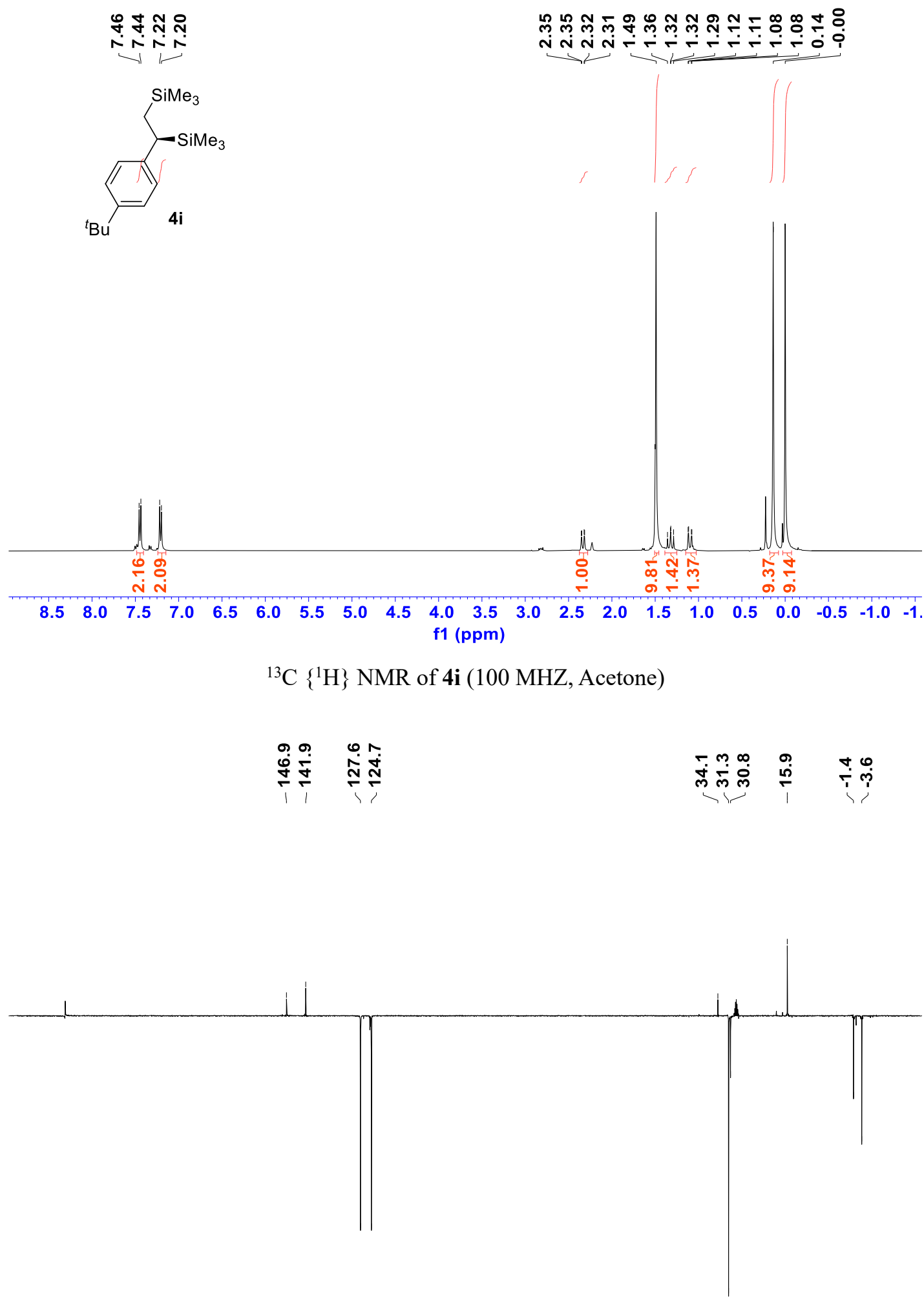

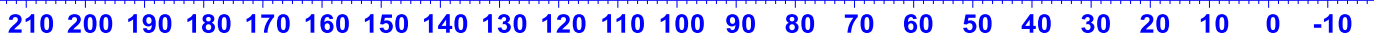
f1 (ppm) 
${ }^{1} \mathrm{H}$ NMR of $\mathbf{4 j}$ (400 MHZ, Acetone)

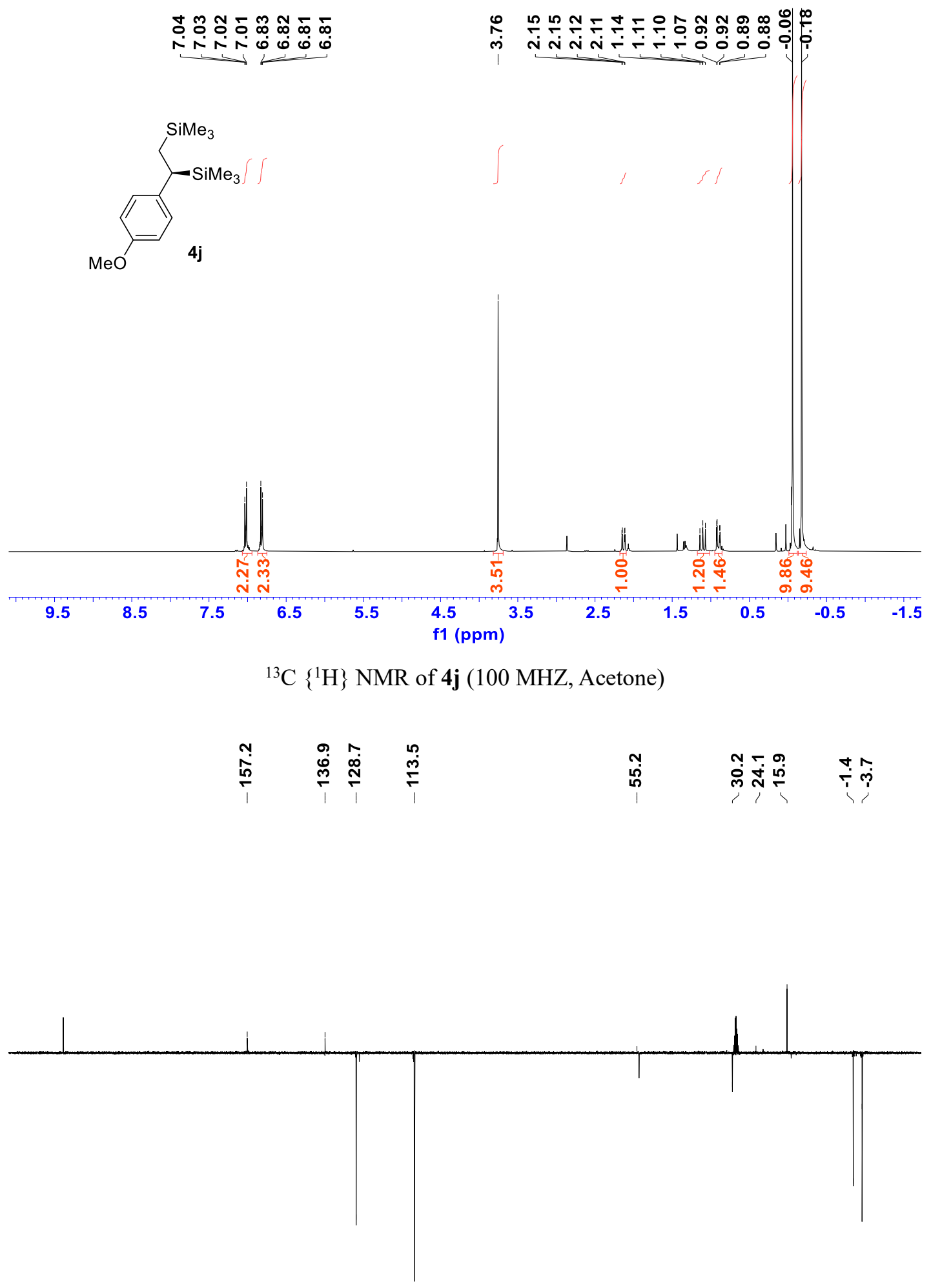

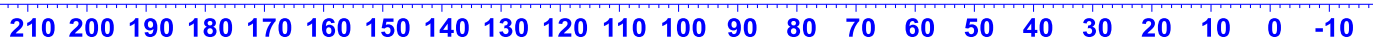
f1 (ppm) 
${ }^{1} \mathrm{H}$ NMR of 4k (400 MHZ, Acetone)

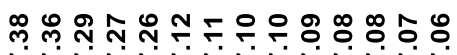

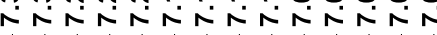

ஸึ

$\sum_{4 \mathbf{k}}^{\mathrm{SiMe}_{3}} \mathrm{SiMe}_{3}$

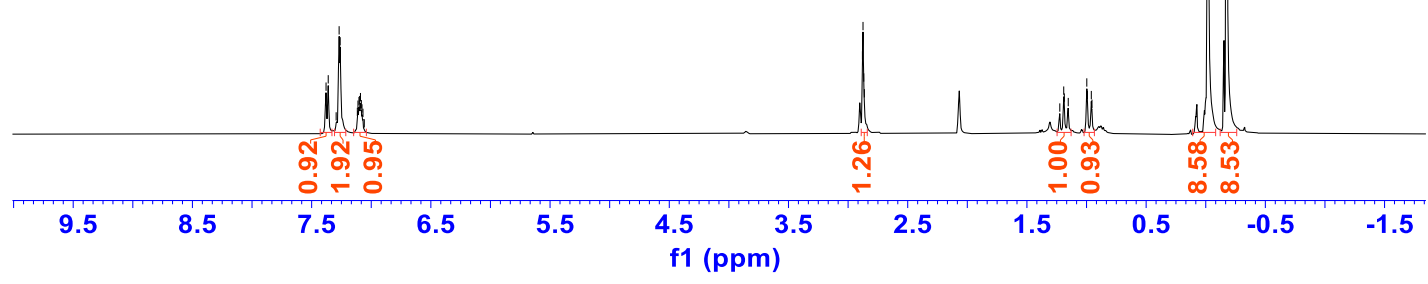

${ }^{13} \mathrm{C}\left\{{ }^{1} \mathrm{H}\right\}$ NMR of $4 \mathbf{k}$ (100 MHZ, Acetone)

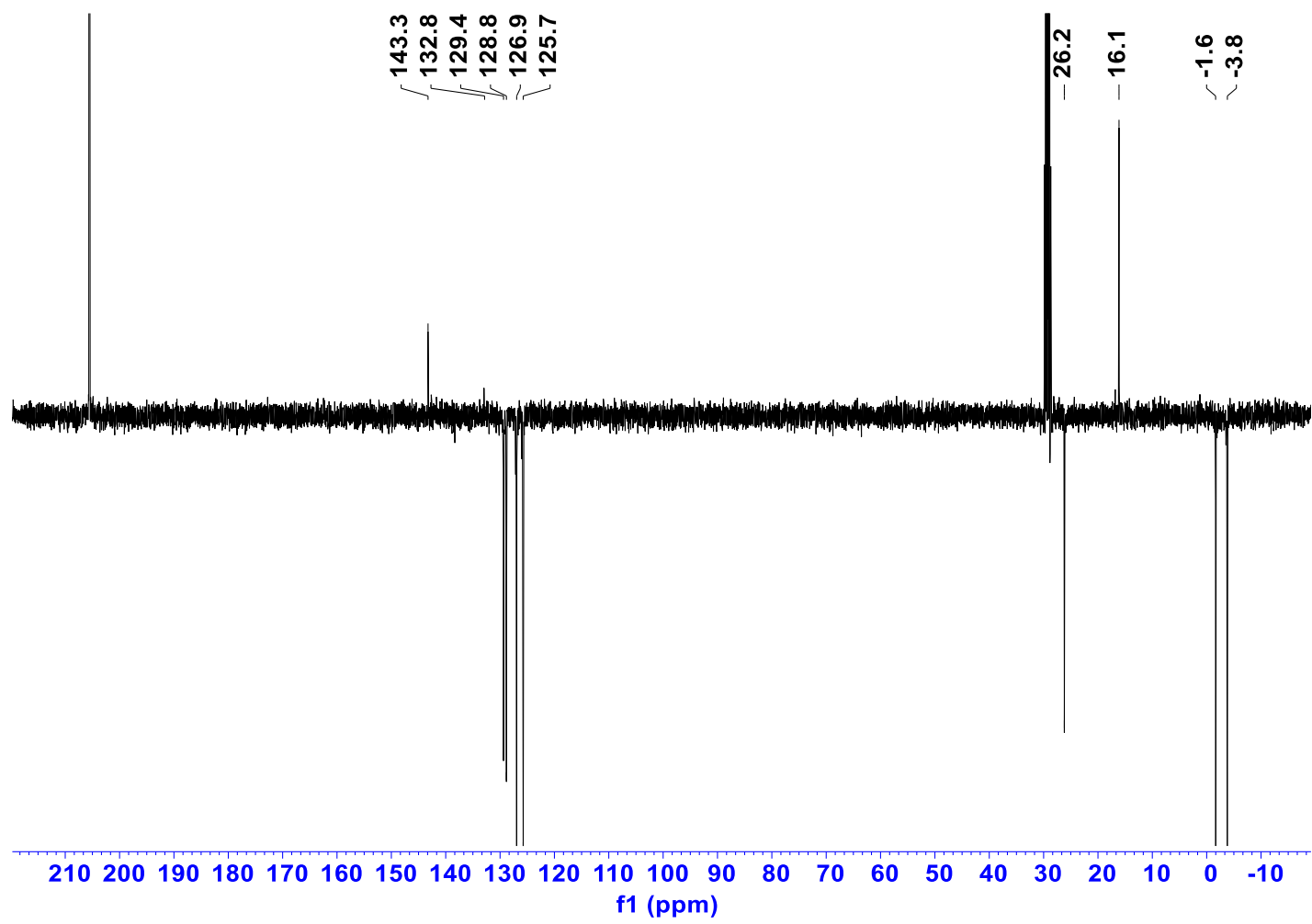


${ }^{1} \mathrm{H}$ NMR of $\mathbf{4 l}$ (400 MHZ, Acetone)

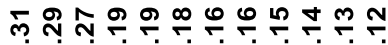

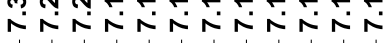

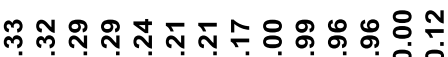

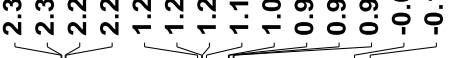

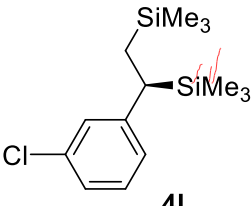

4!

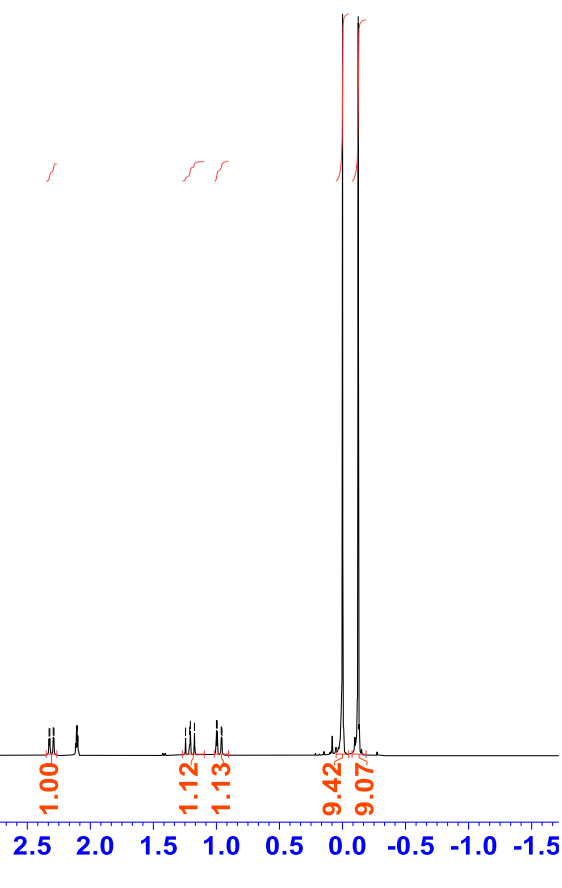

${ }^{13} \mathrm{C}\left\{{ }^{1} \mathrm{H}\right\}$ NMR of $\mathbf{4 l}$ (100 MHZ, Acetone)
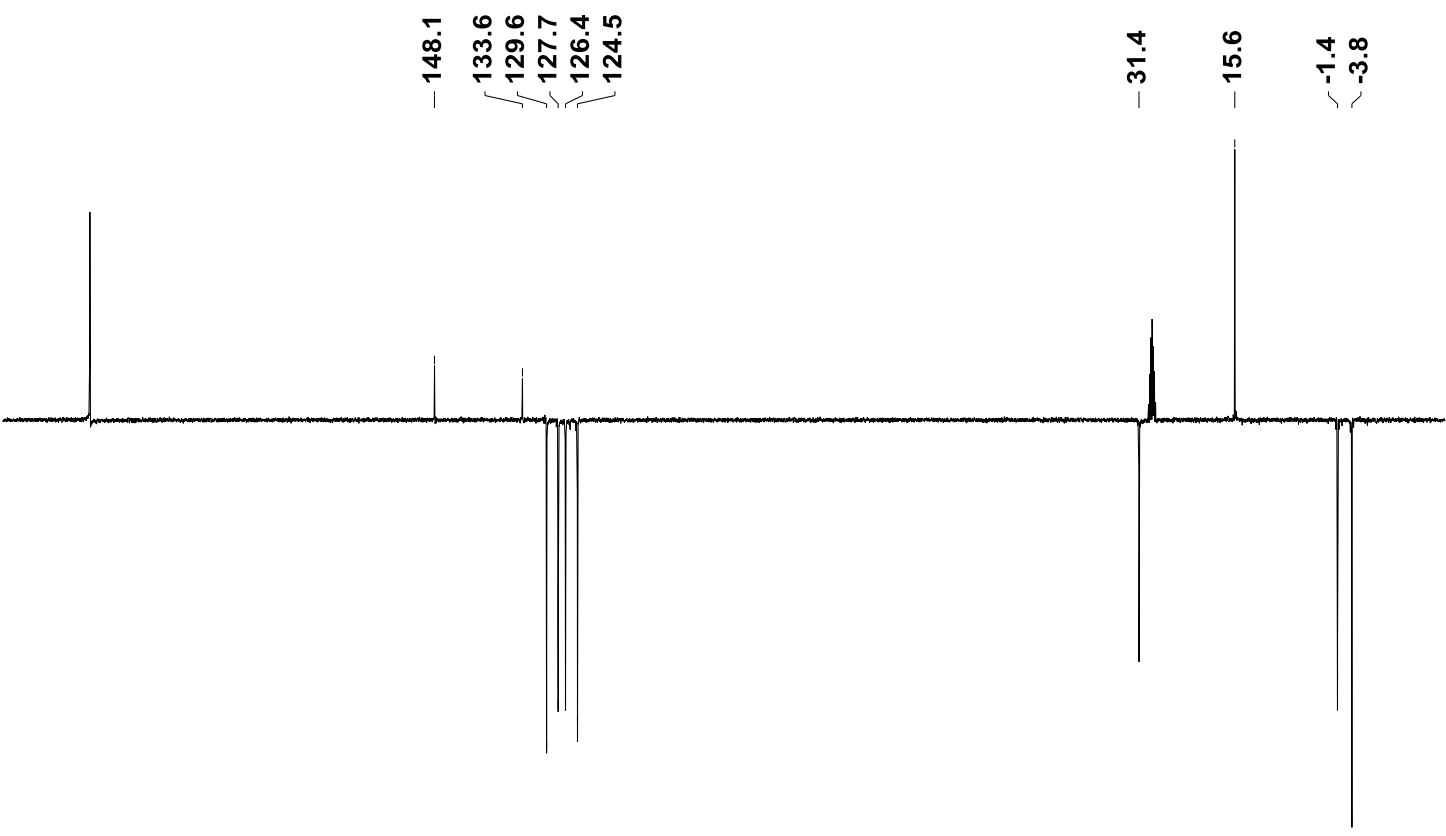

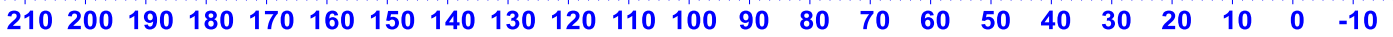
f1 (ppm) 
${ }^{1} \mathrm{H}$ NMR of $4 \mathbf{m}$ (400 MHZ, Acetone)

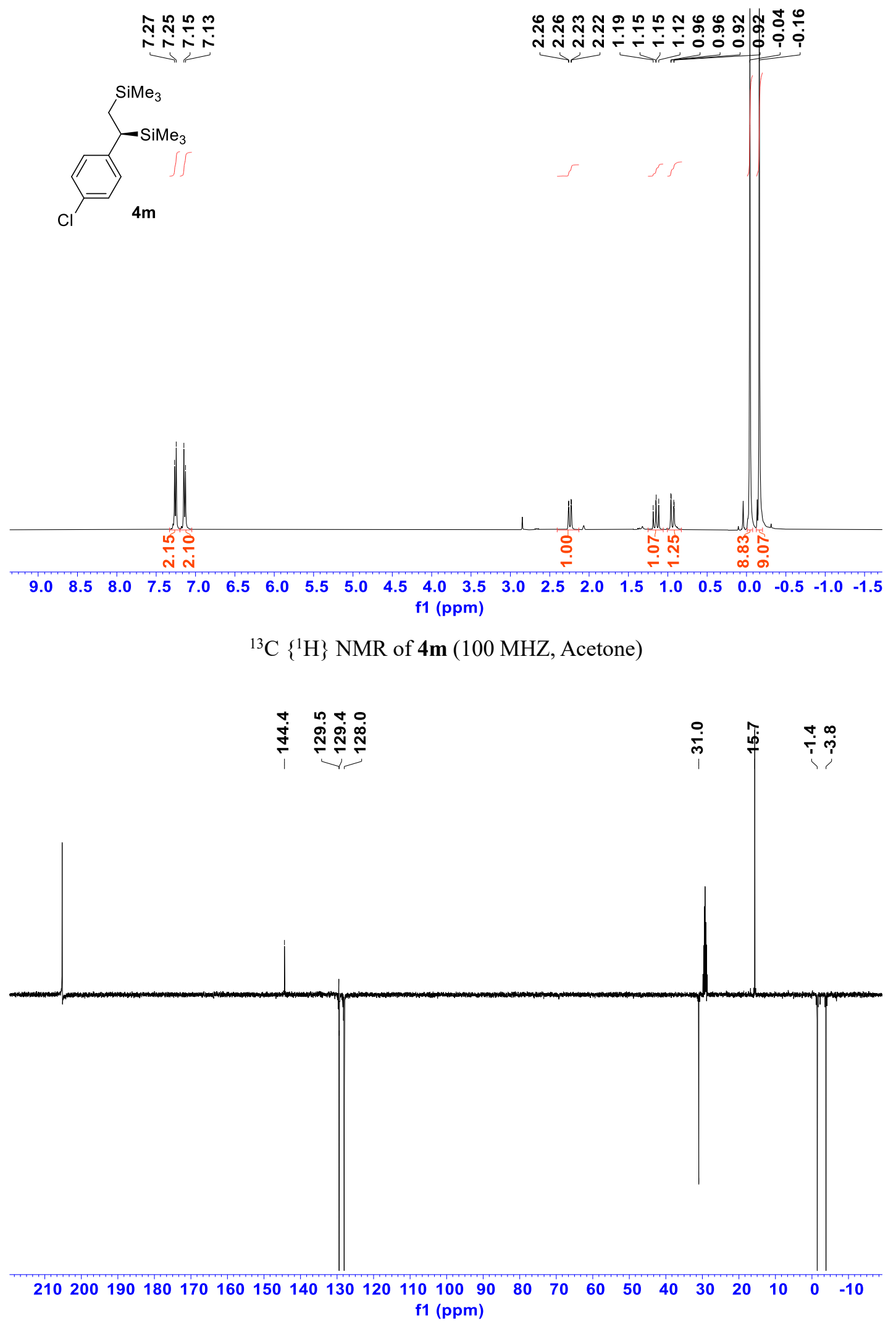


${ }^{1} \mathrm{H}$ NMR of 4n (400 MHZ, Acetone)

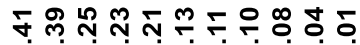

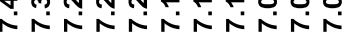

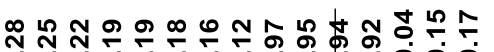

h<smiles>CCC(C)c1ccc(Br)cc1</smiles>

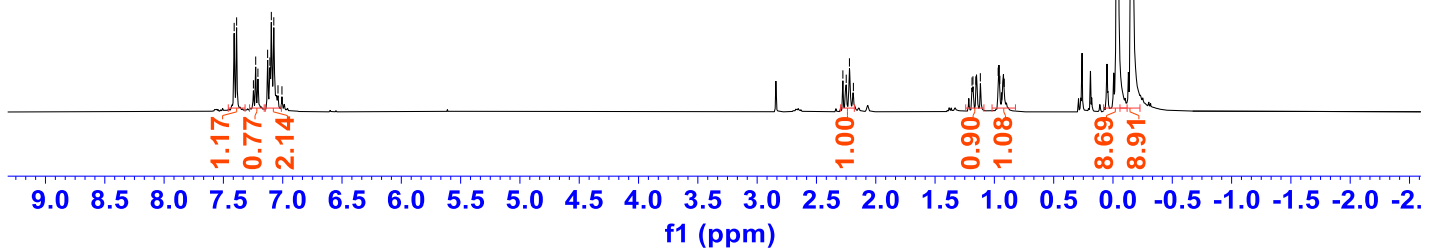

${ }^{13} \mathrm{C}\left\{{ }^{1} \mathrm{H}\right\}$ NMR of $4 \mathbf{n}(100 \mathrm{MHZ}$, Acetone)
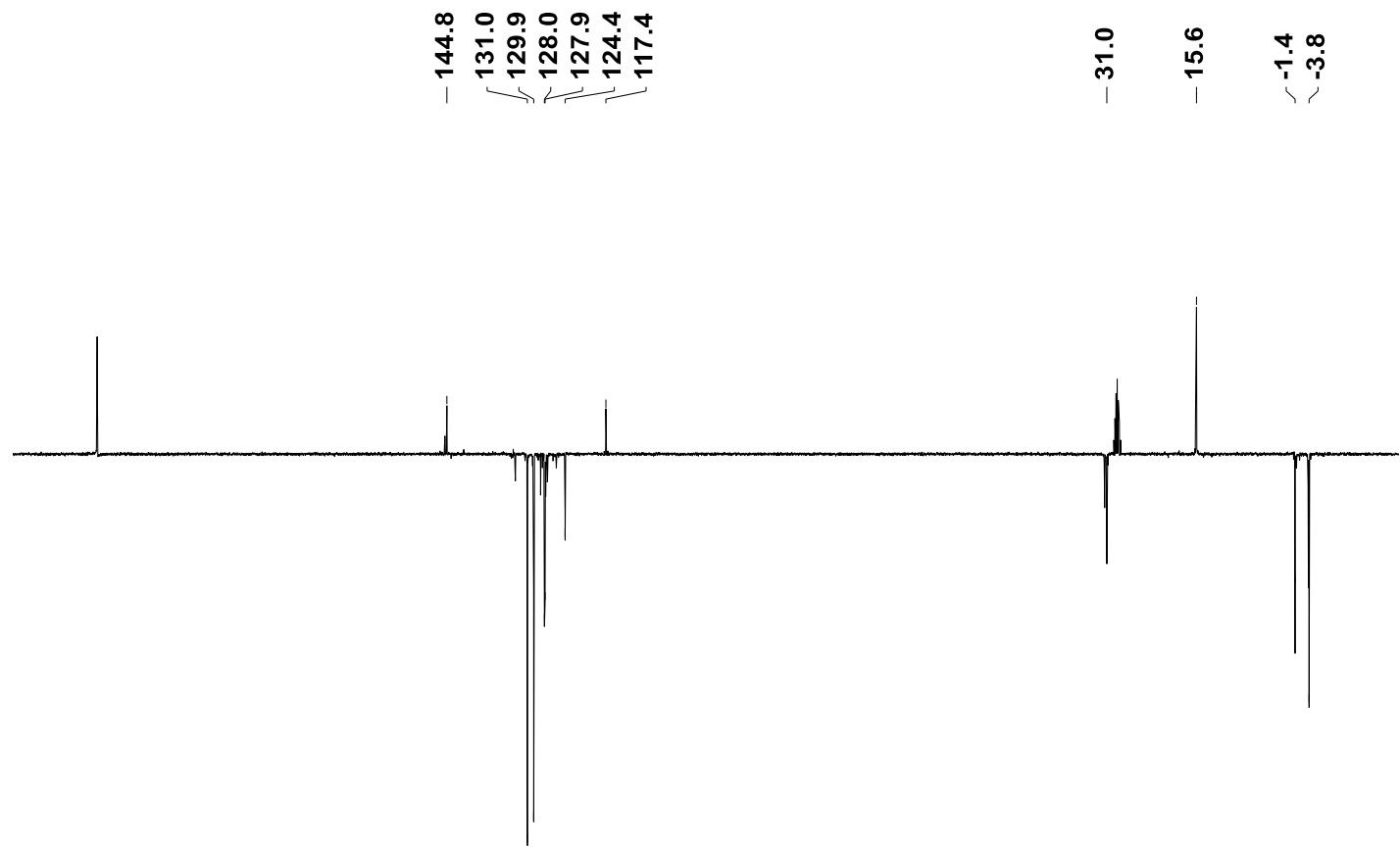

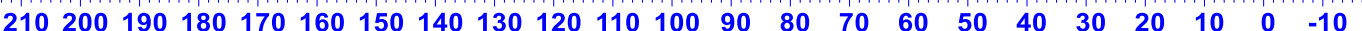
f1 (ppm) 
${ }^{1} \mathrm{H}$ NMR of 40 (400 MHZ, Acetone)

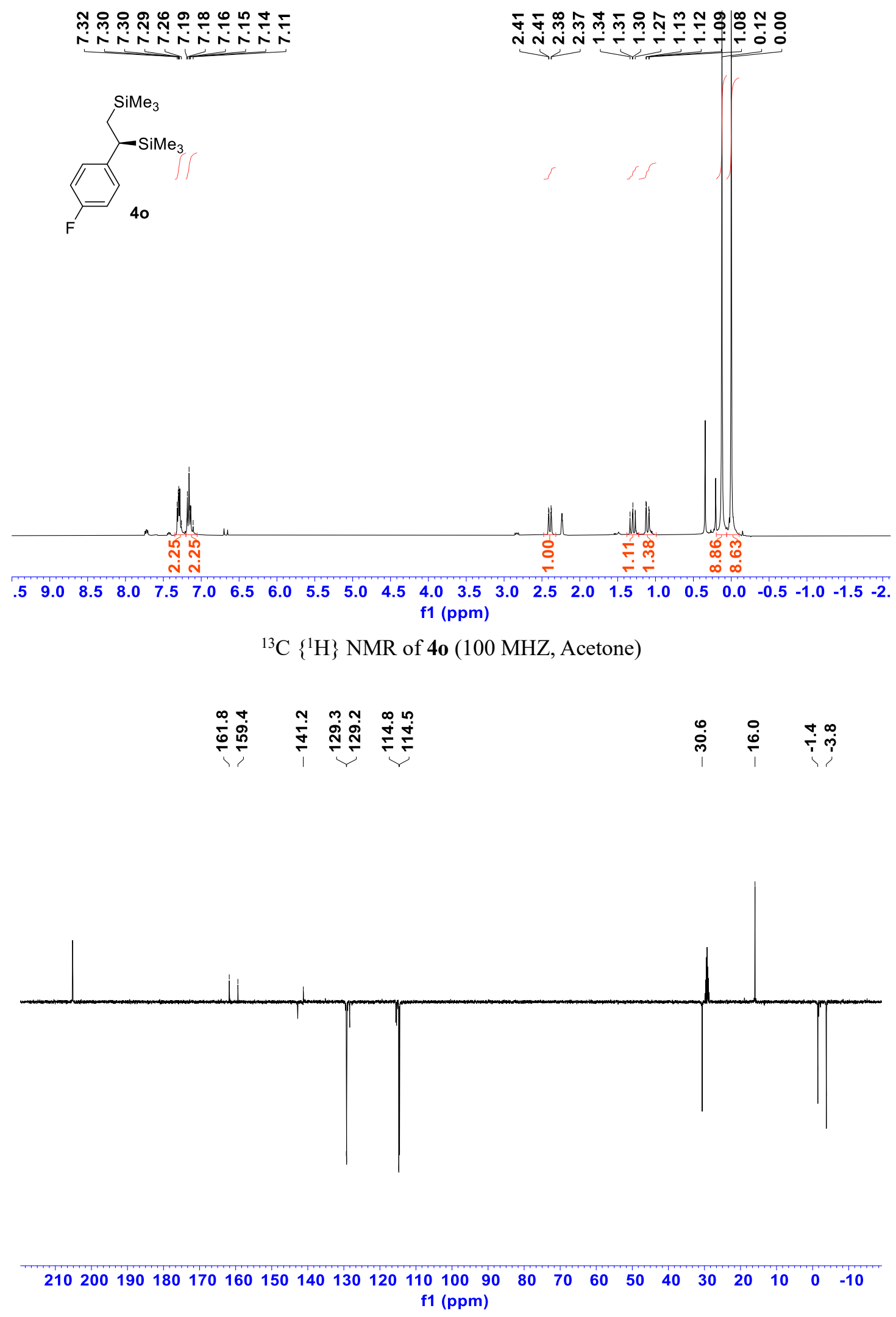


${ }^{19} \mathrm{~F}$ NMR of $40\left(377 \mathrm{MHZ}, \mathrm{CDCl}_{3}\right)$

ํํํ

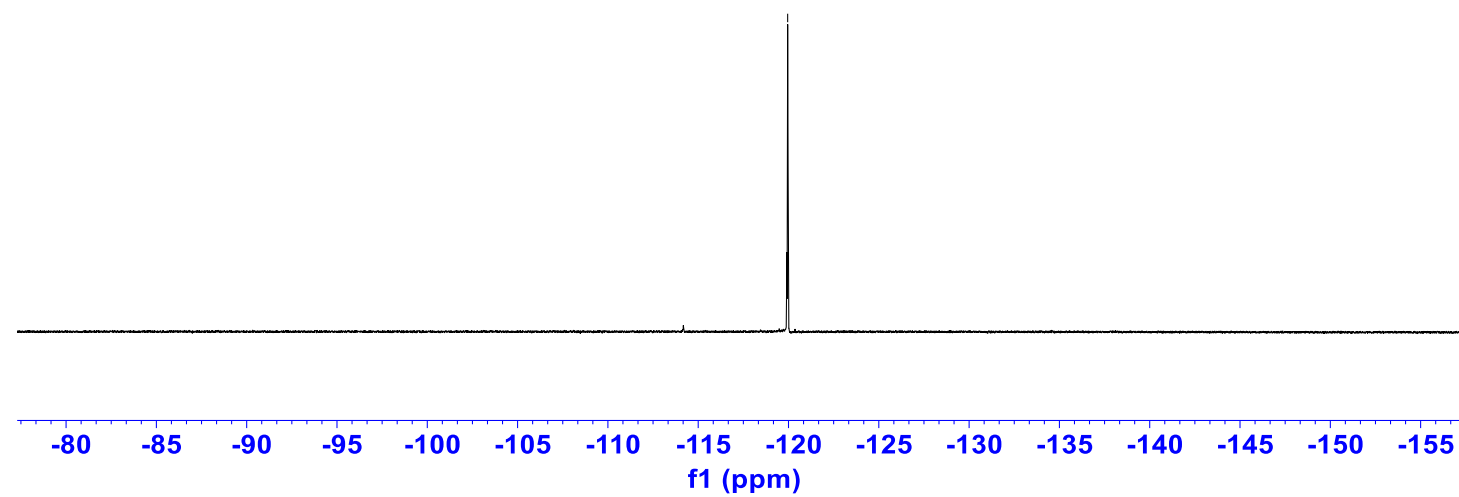


${ }^{1} \mathrm{H}$ NMR of 4p (400 MHZ, Acetone)

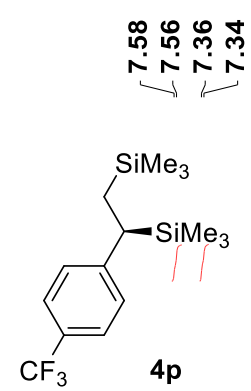

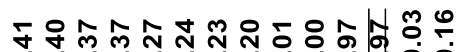
ก n 望

令垈 nnn n- $n$
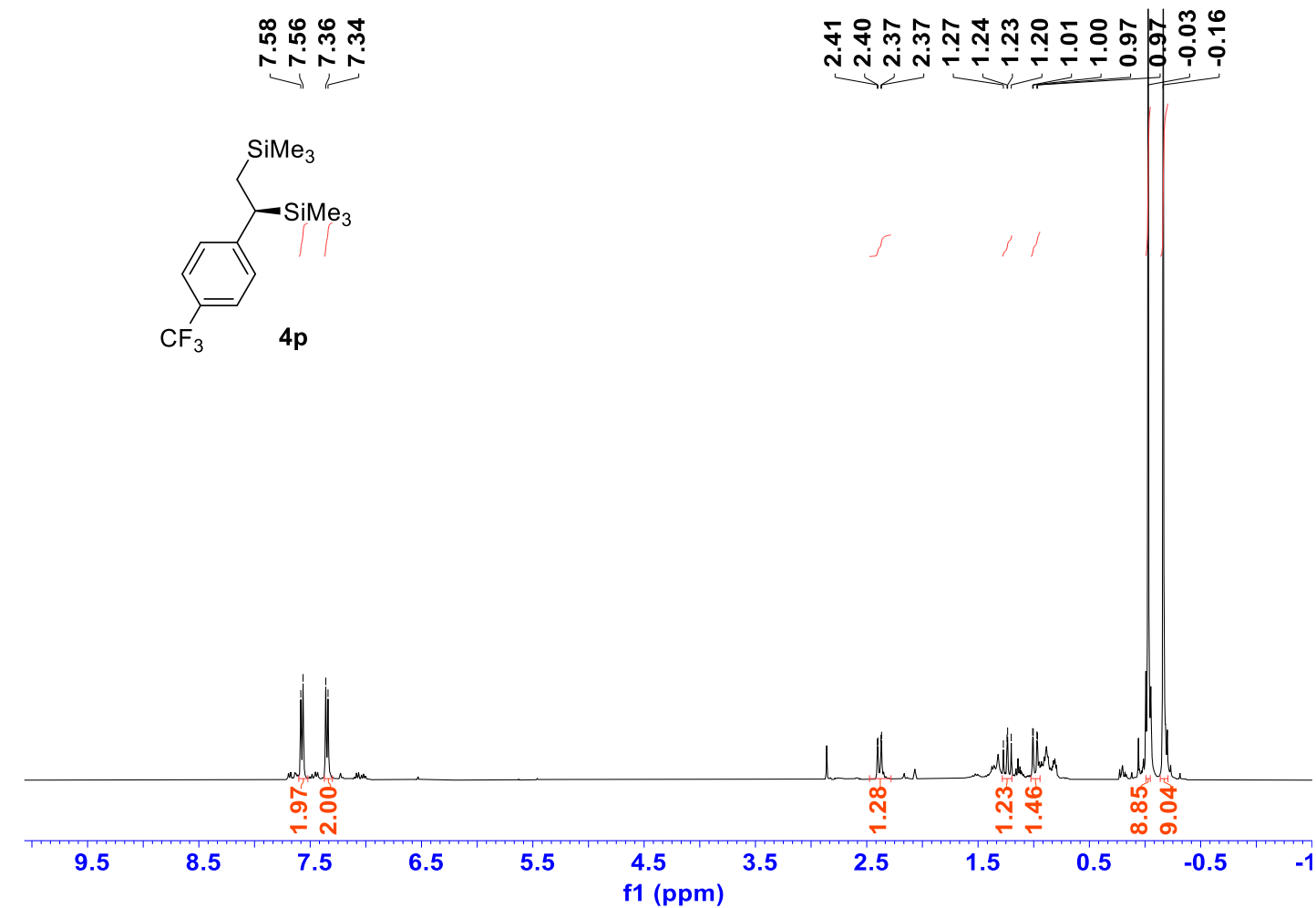

${ }^{13} \mathrm{C}\left\{{ }^{1} \mathrm{H}\right\}$ NMR of $\mathbf{4 p}(100 \mathrm{MHZ}$, Acetone)

눙

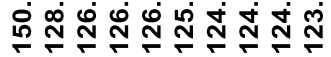

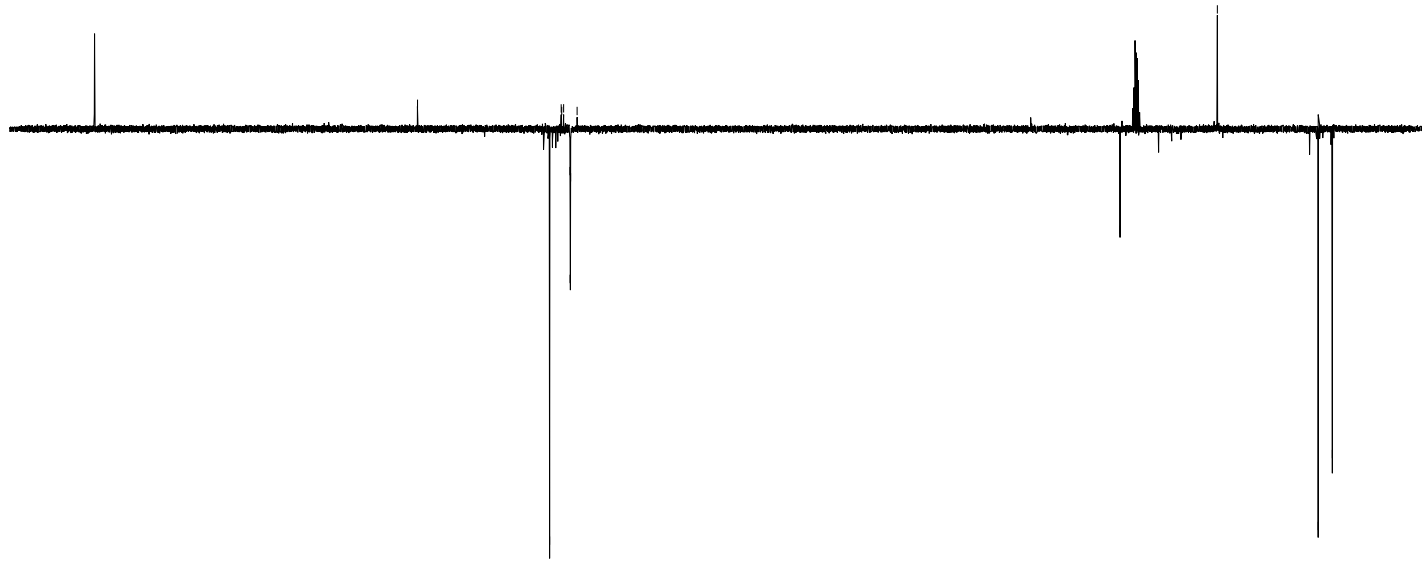


${ }^{19} \mathrm{~F}$ NMR of $4 p\left(377 \mathrm{MHZ}, \mathrm{CDCl}_{3}\right)$

$\stackrel{\circ}{\text { ị }}$

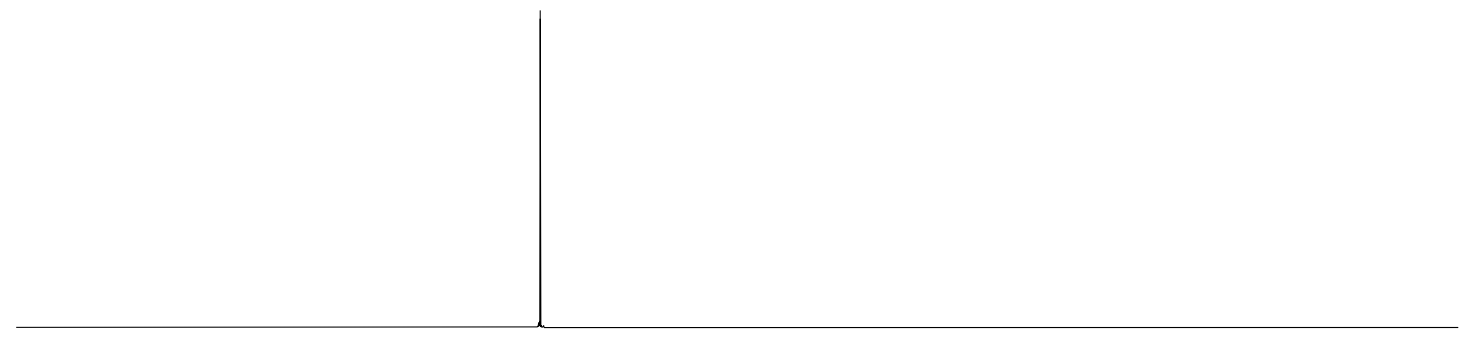

\begin{tabular}{llllllllllllllllllllllllllllll}
\hline 10 & 0 & -10 & -20 & -30 & -40 & -50 & -60 & -70 & -80 & -90 & -100 & -110 & -120 & -130 & -140 & -150 & -160 & -170 & -180 & -19
\end{tabular} f1 (ppm) 
${ }^{1} \mathrm{H}$ NMR of 4q (400 MHZ, Acetone)

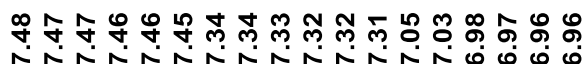

ֻุ

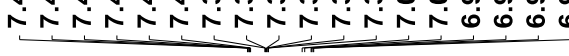

nising
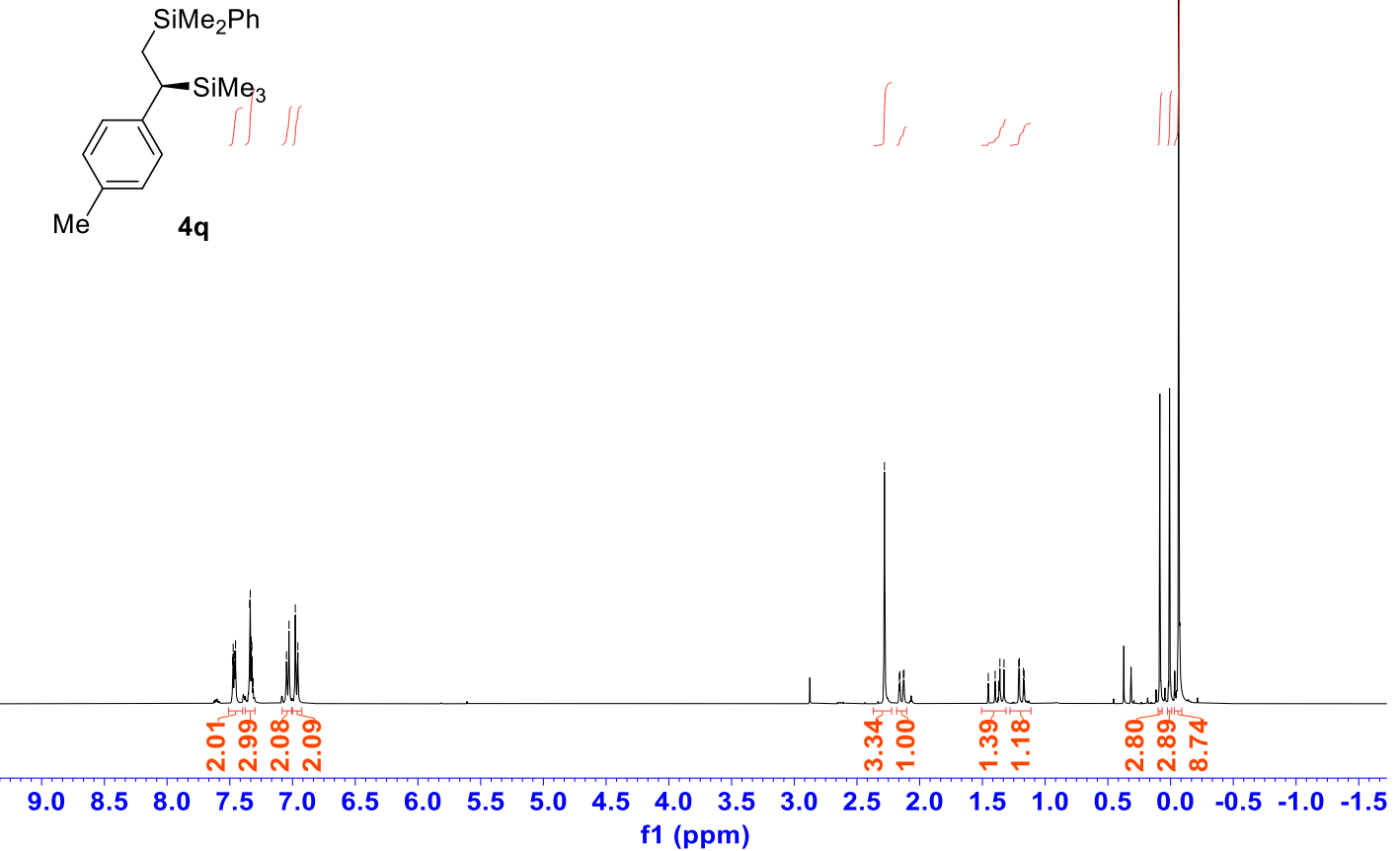

${ }^{13} \mathrm{C}\left\{{ }^{1} \mathrm{H}\right\}$ NMR of $\mathbf{4 q}(100 \mathrm{MHZ}$, Acetone)

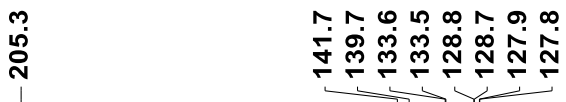

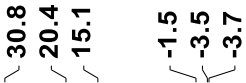

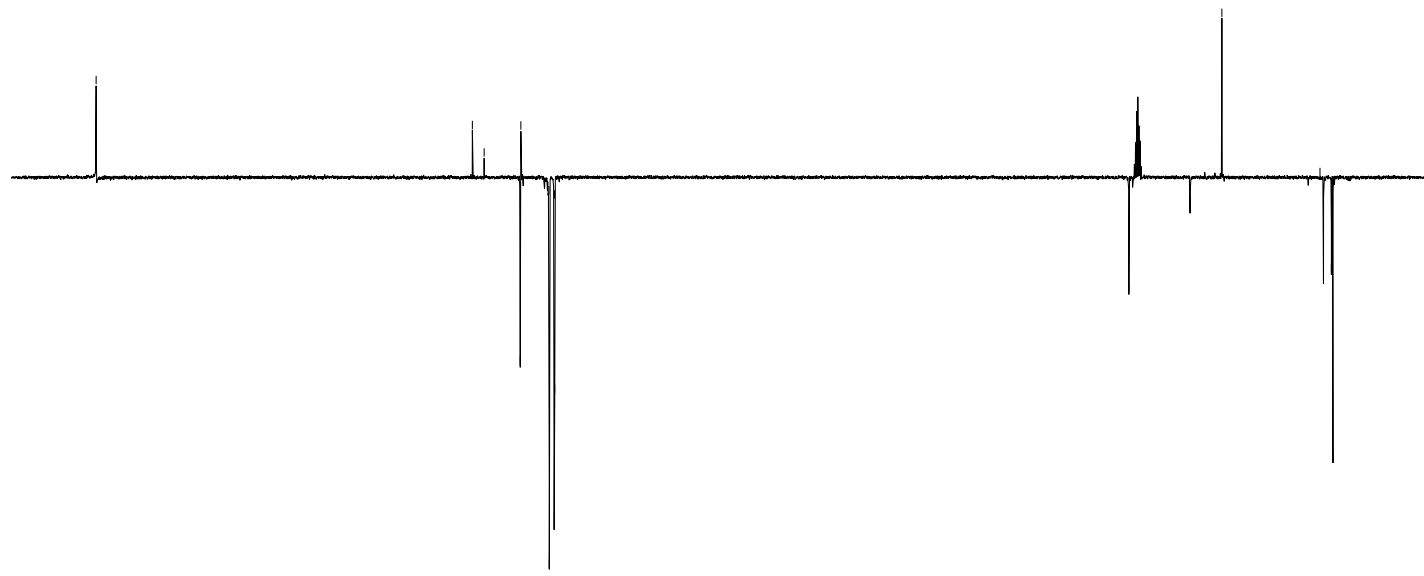

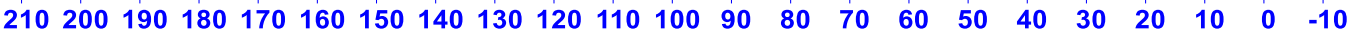
f1 (ppm) 
${ }^{1} \mathrm{H}$ NMR of $4 \mathbf{r}(400 \mathrm{MHZ}$, Acetone)

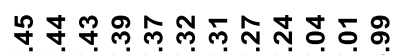

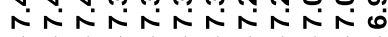

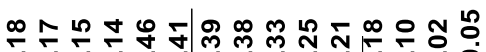

$\mathrm{SiMe}_{3}$
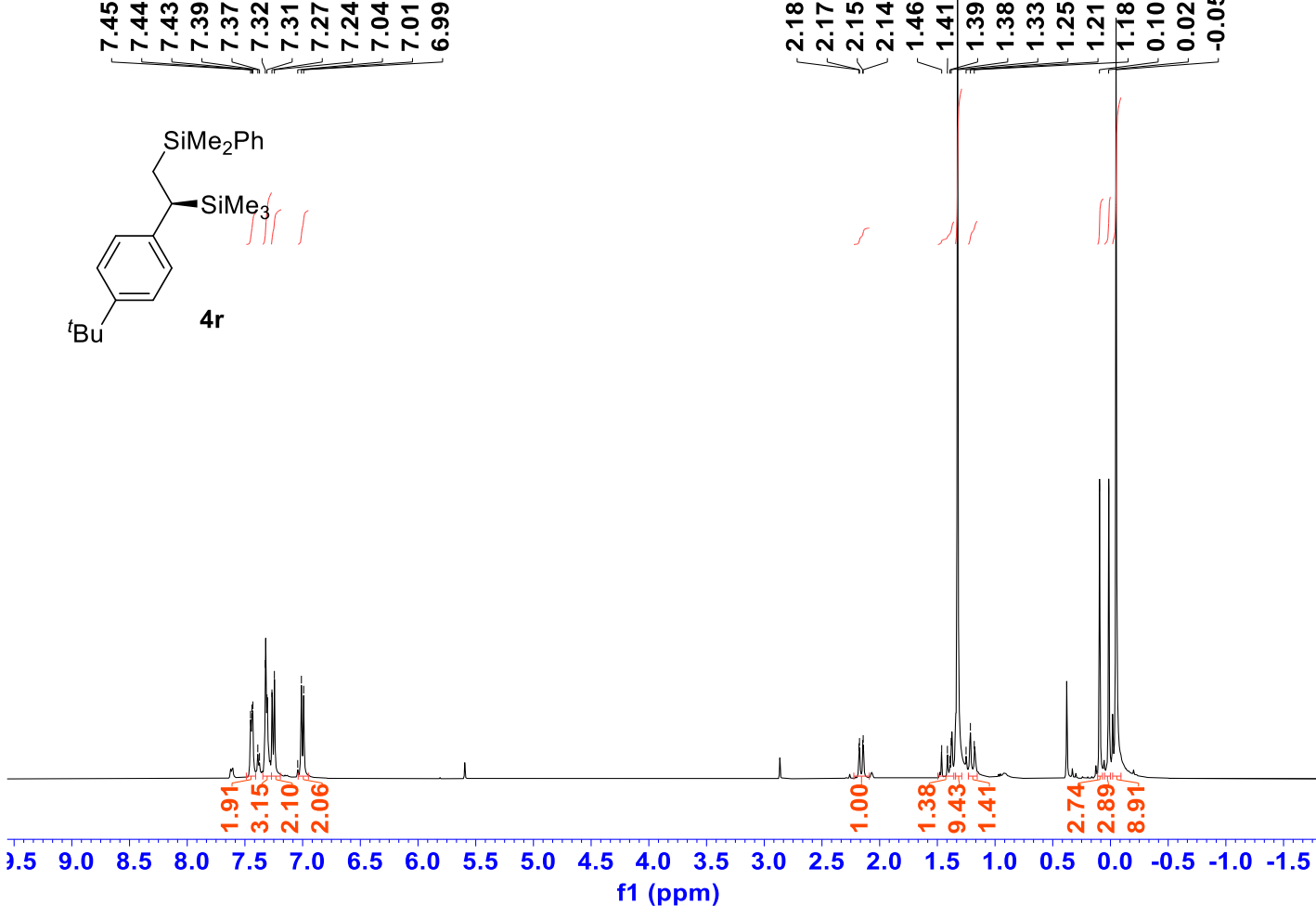

${ }^{13} \mathrm{C}\left\{{ }^{1} \mathrm{H}\right\}$ NMR of $4 \mathbf{r}(100 \mathrm{MHZ}$, Acetone)

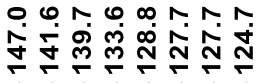

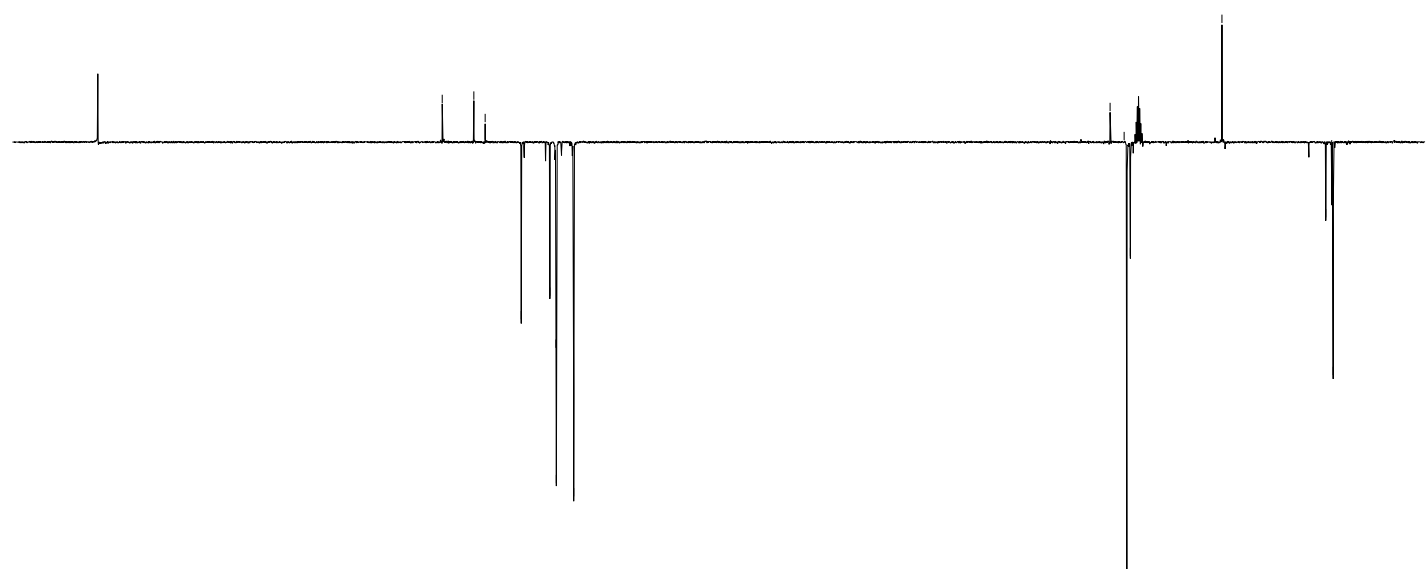

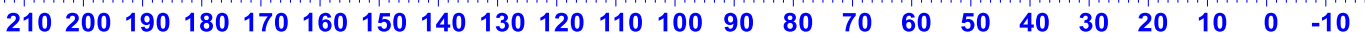
f1 (ppm) 
${ }^{1} \mathrm{H}$ NMR of 4s (400 MHZ, Acetone)

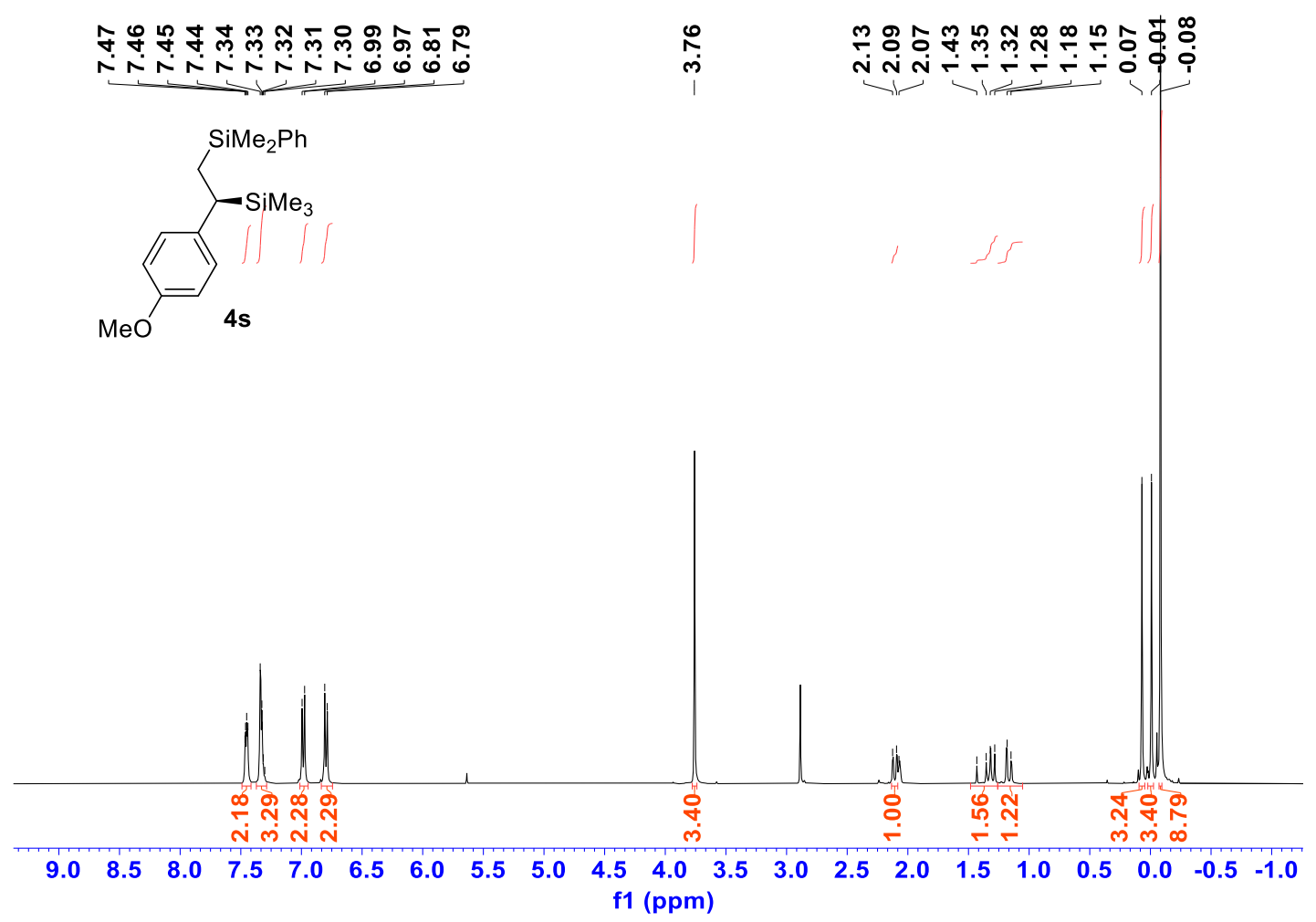

${ }^{13} \mathrm{C}\left\{{ }^{1} \mathrm{H}\right\}$ NMR of $4 \mathbf{s}(100 \mathrm{MHZ}$, Acetone)

蓆

总

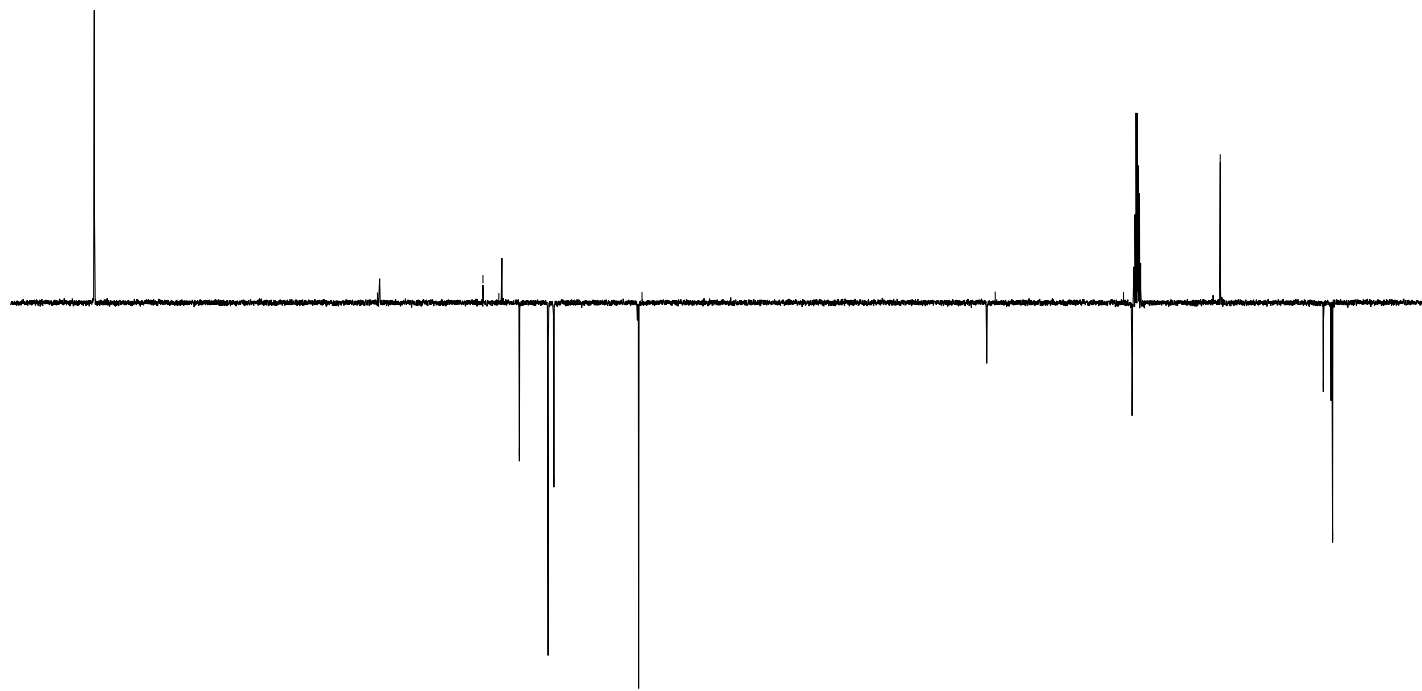

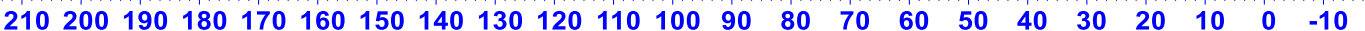
f1 (ppm) 
${ }^{1} \mathrm{H}$ NMR of $4 \mathbf{t}$ (400 MHZ, Acetone)

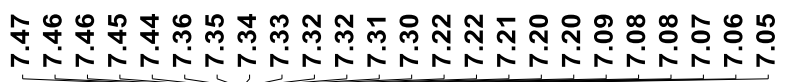

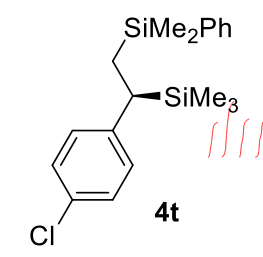

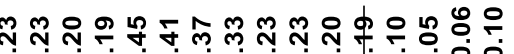
h

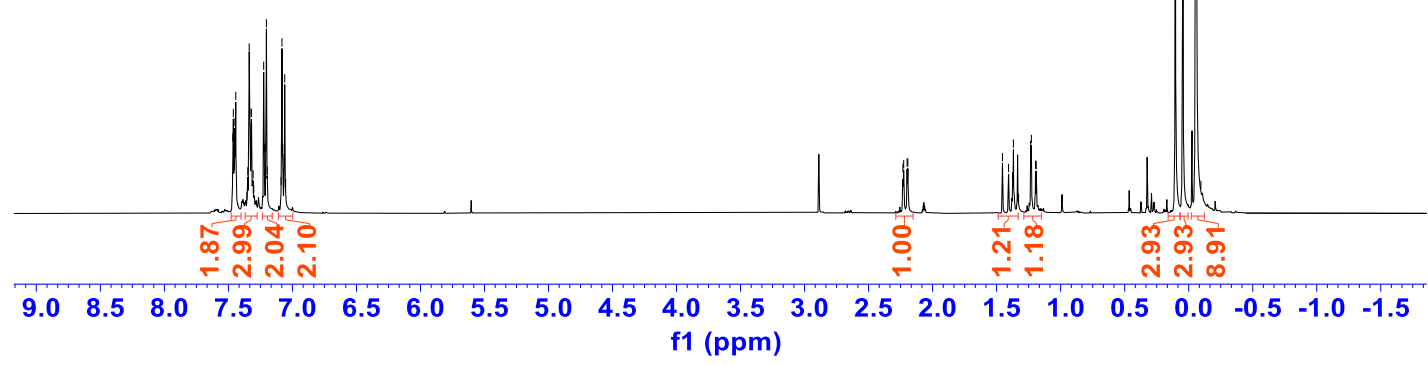

${ }^{13} \mathrm{C}\left\{{ }^{1} \mathrm{H}\right\}$ NMR of $4 \mathbf{t}$ (100 MHZ, Acetone)

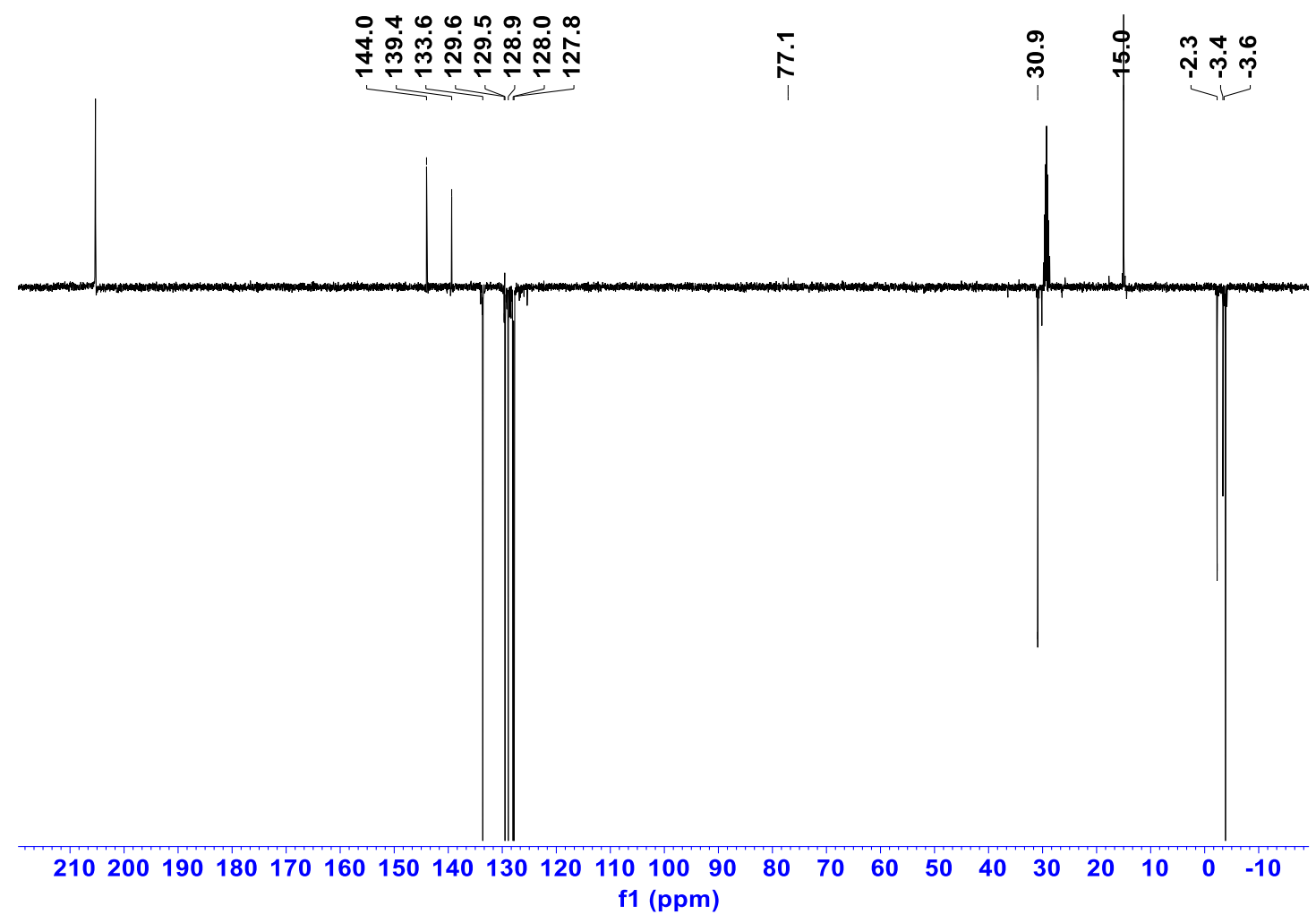


${ }^{1} \mathrm{H}$ NMR of $4 \mathbf{u}$ (400 MHZ, Acetone)

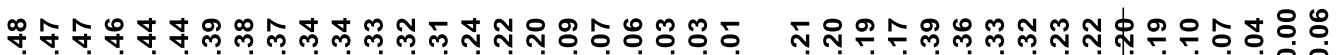

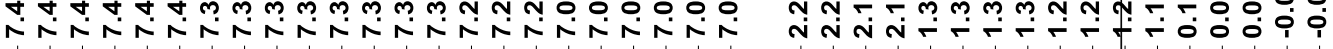<smiles>CC(C)CC(C)c1ccc(Br)cc1</smiles>

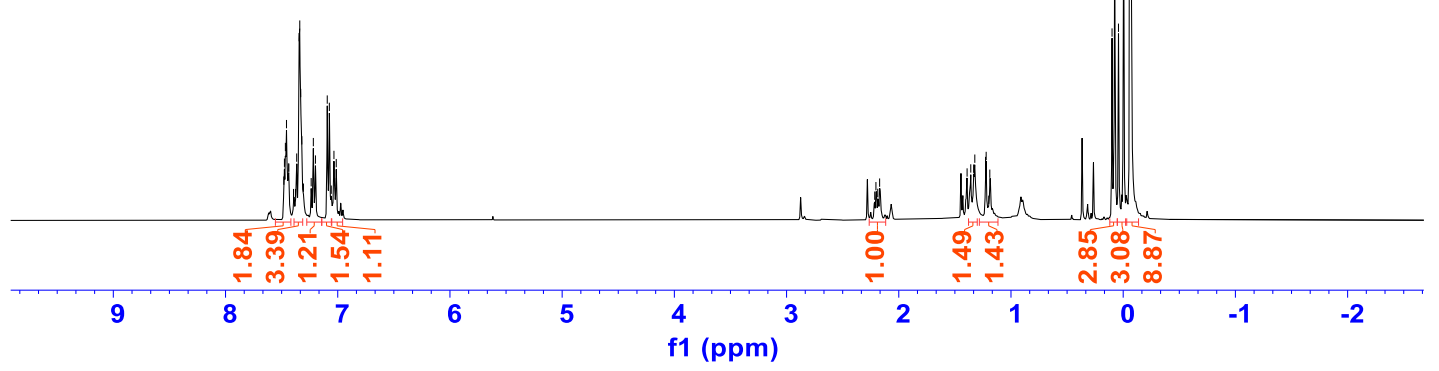

${ }^{13} \mathrm{C}\left\{{ }^{1} \mathrm{H}\right\}$ NMR of $\mathbf{4 u}(100 \mathrm{MHZ}$, Acetone)

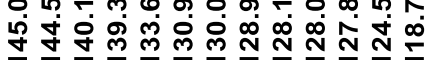

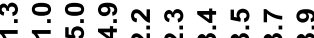

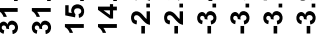

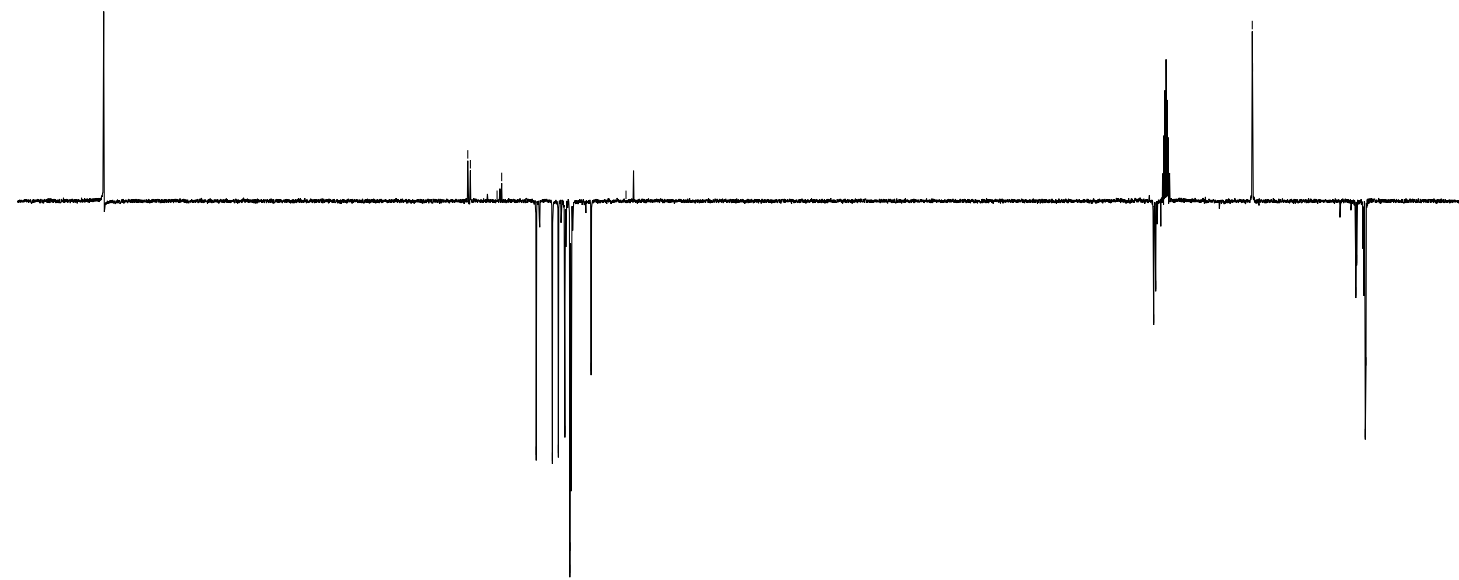

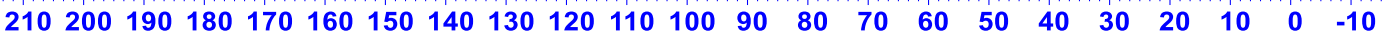
f1 (ppm) 
${ }^{1} \mathrm{H}$ NMR of $4 \mathbf{v}$ (400 MHZ, Acetone)

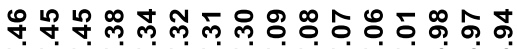

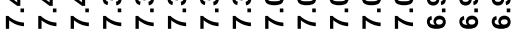

ฟุ

ñ
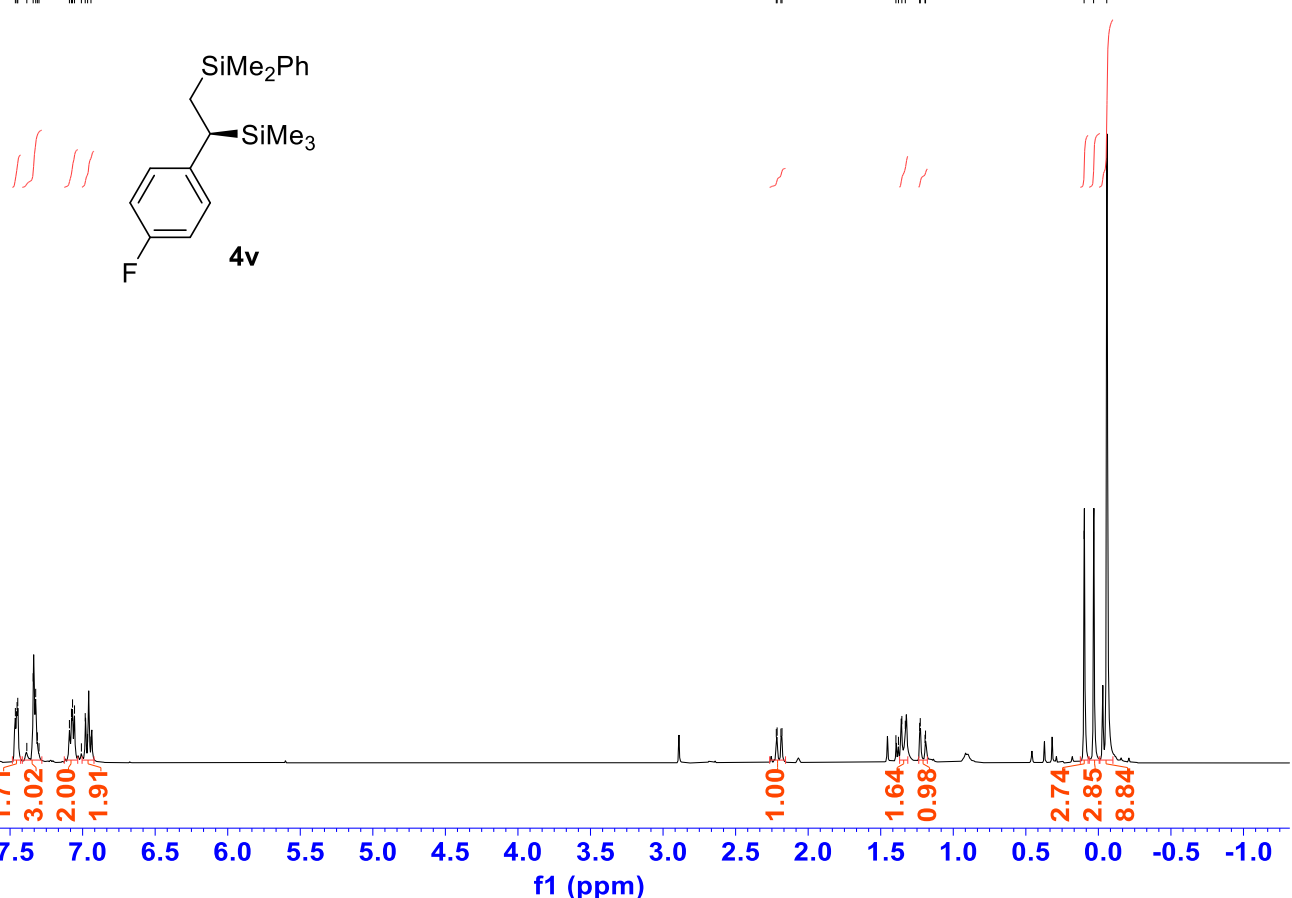

${ }^{13} \mathrm{C}\left\{{ }^{1} \mathrm{H}\right\}$ NMR of $\mathbf{4 v}(100 \mathrm{MHZ}$, Acetone)

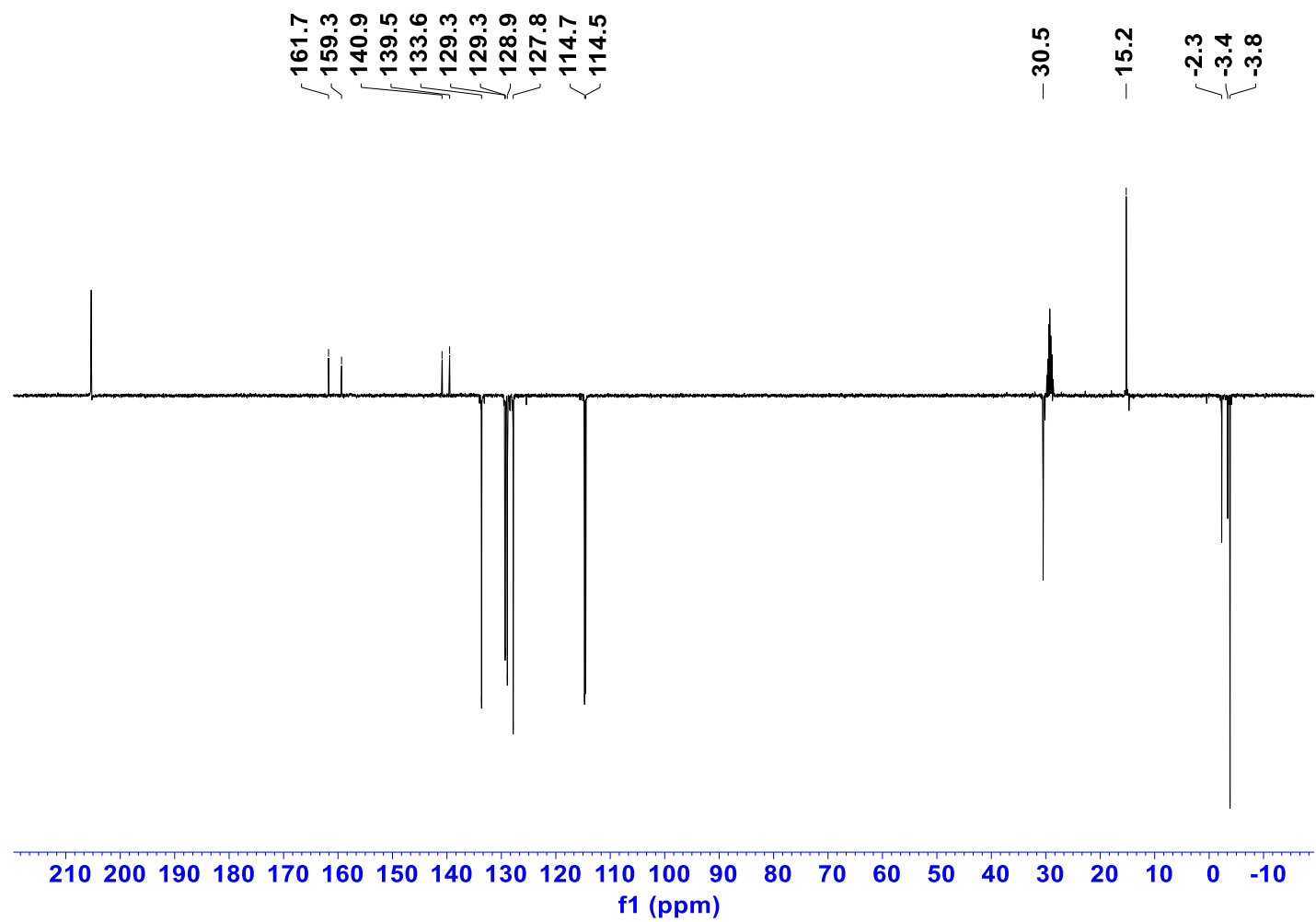

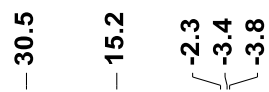

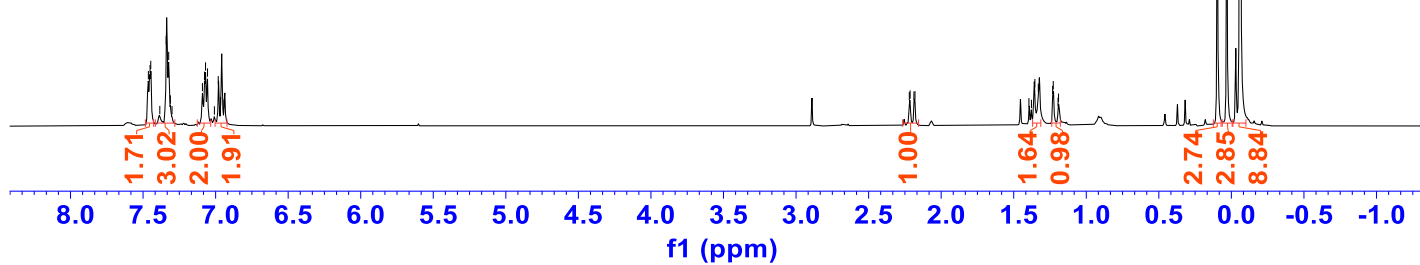


${ }^{19} \mathrm{~F}$ NMR of $4 \mathbf{v}\left(377 \mathrm{MHZ}, \mathrm{CDCl}_{3}\right)$

$\stackrel{9}{9}$

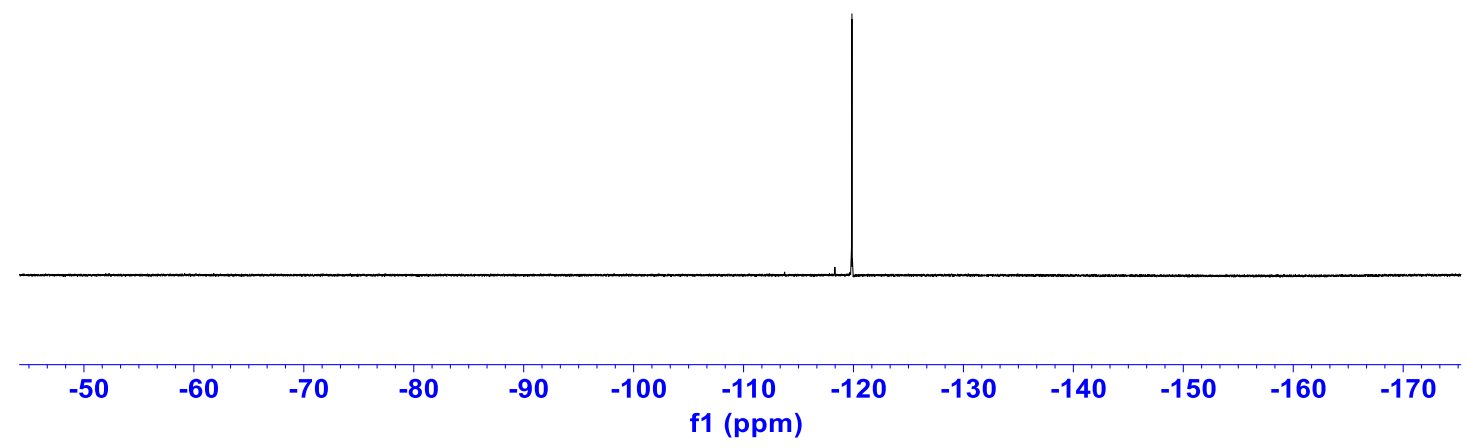


${ }^{1} \mathrm{H}$ NMR of $\mathbf{4 w}$ (400 MHZ, Acetone)

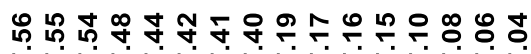

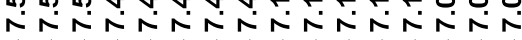

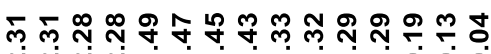

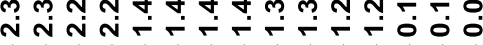

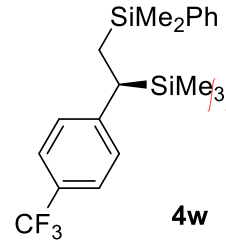

w

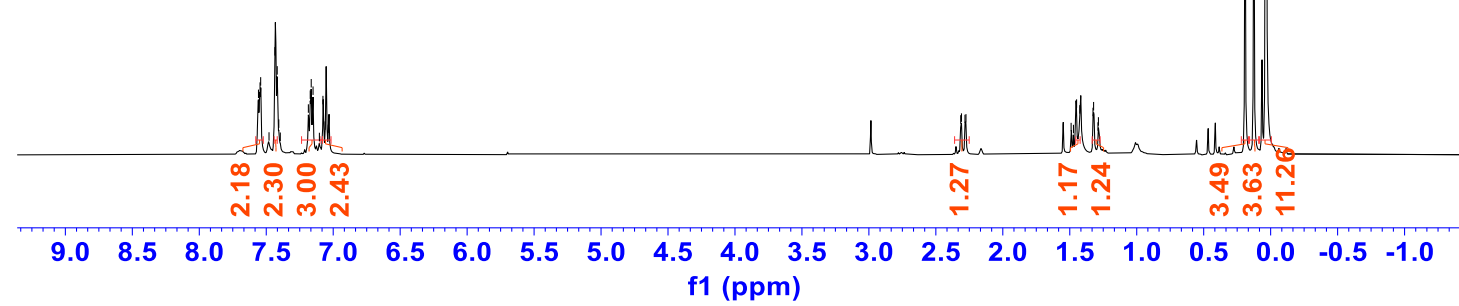

${ }^{13} \mathrm{C}\left\{{ }^{1} \mathrm{H}\right\}$ NMR of 4w (100 MHZ, Acetone)



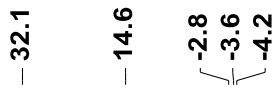

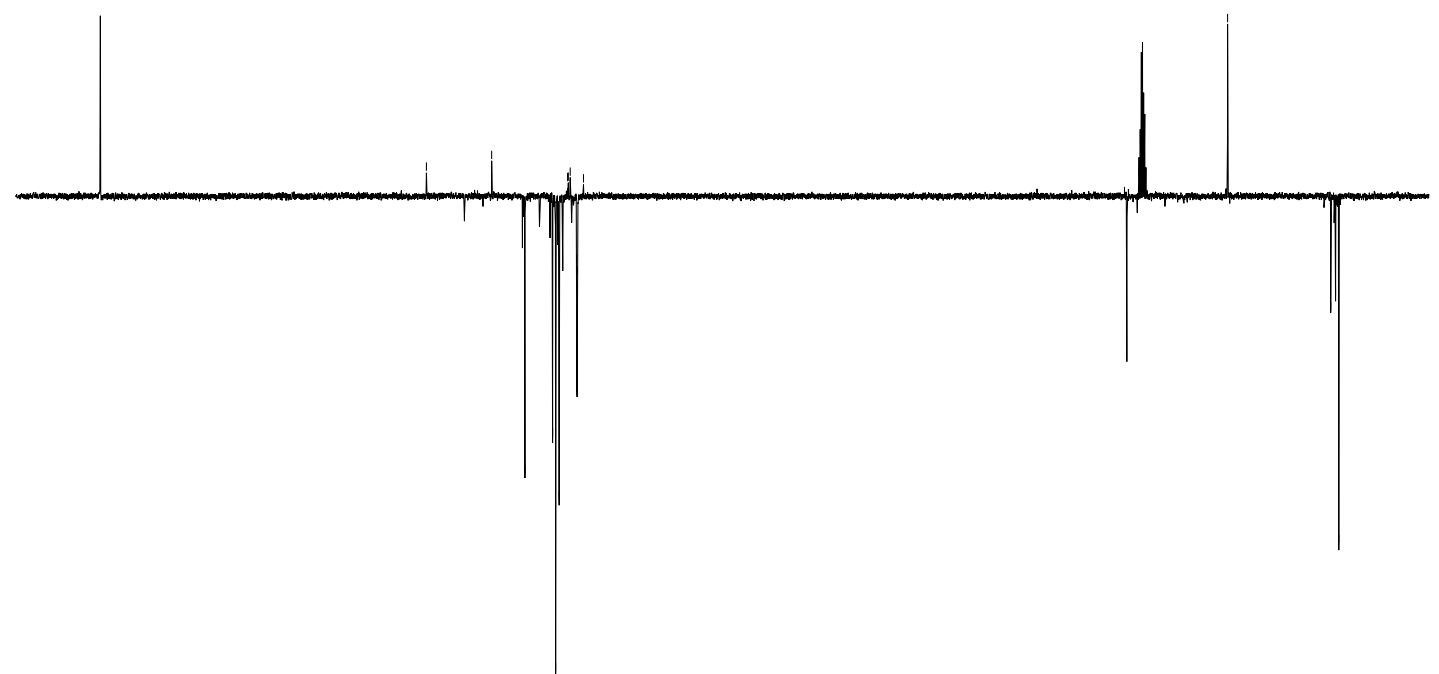

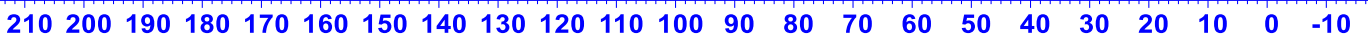
f1 (ppm) 
${ }^{19} \mathrm{~F}$ NMR of $4 \mathbf{w}\left(377 \mathrm{MHZ}, \mathrm{CDCl}_{3}\right)$

$\frac{9}{0}$

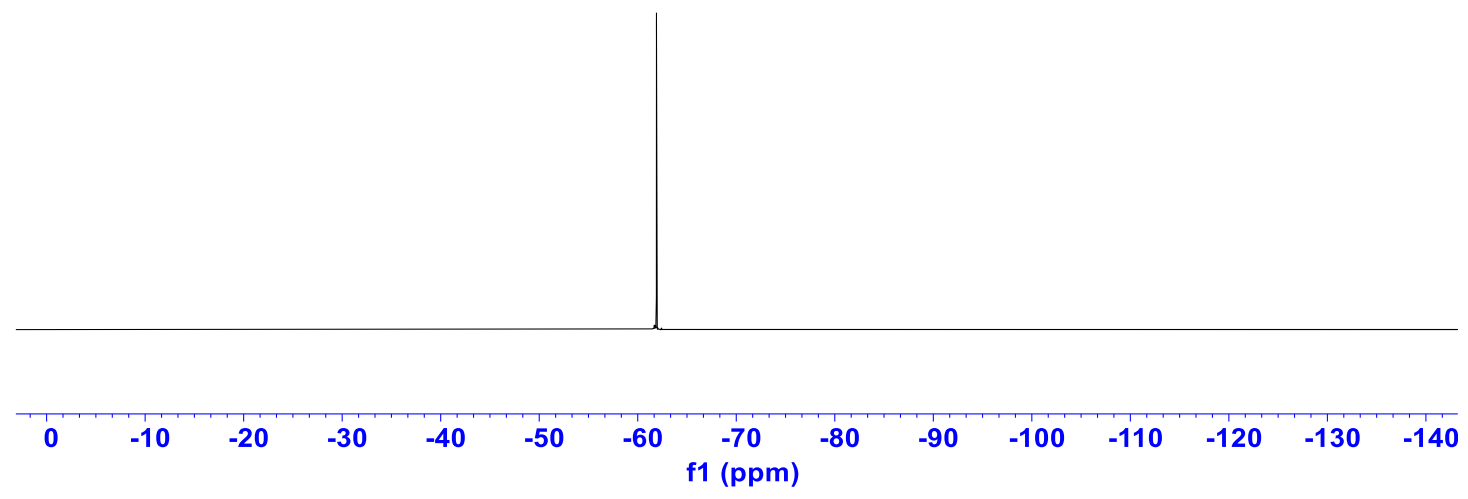


${ }^{1} \mathrm{H}$ NMR of $\mathbf{L} 7\left(400 \mathrm{MHZ}, \mathrm{CDCl}_{3}\right)$

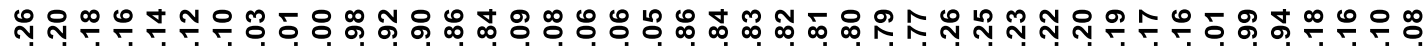

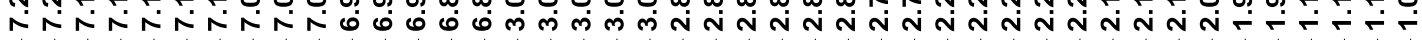
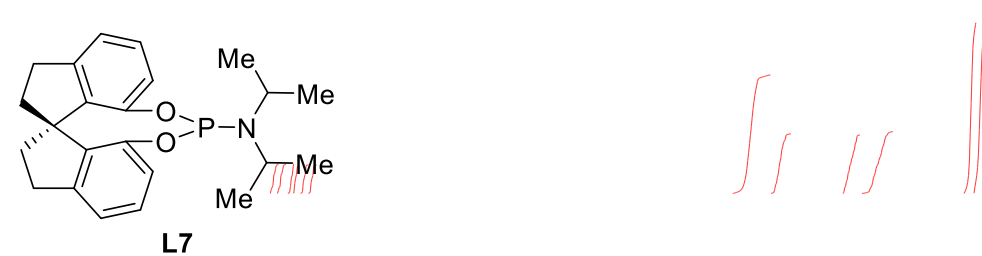

L7

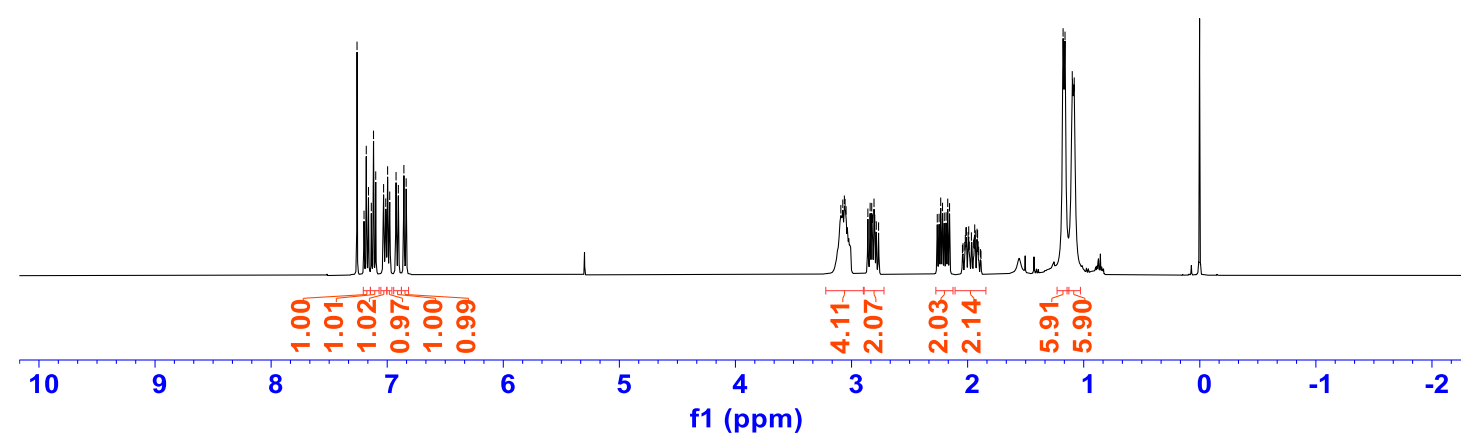

${ }^{13} \mathrm{C}\left\{{ }^{1} \mathrm{H}\right\}$ NMR of L7 (100 MHZ, $\left.\mathrm{CDCl}_{3}\right)$

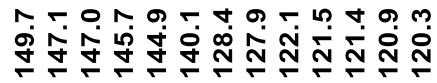

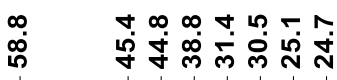

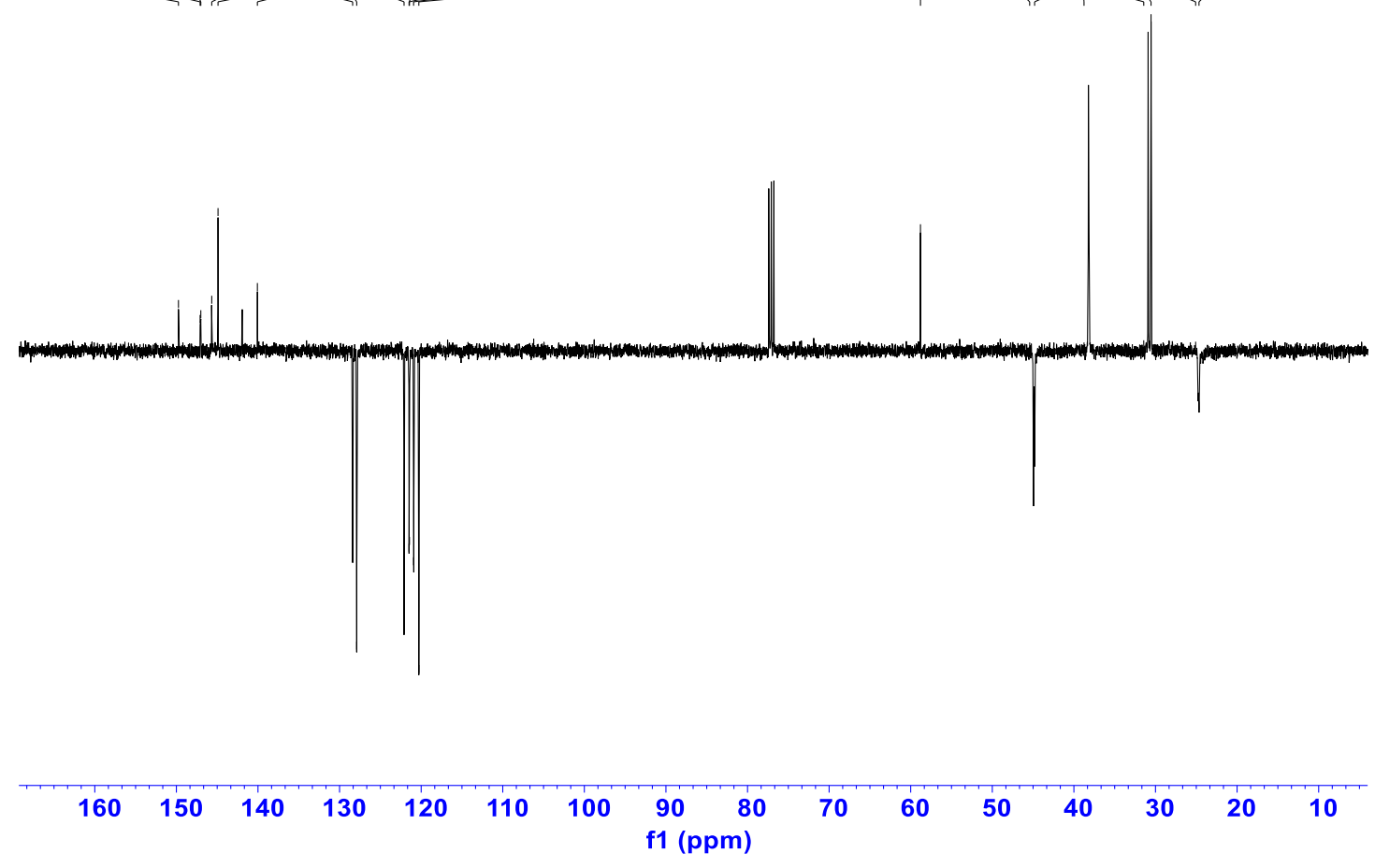


${ }^{31} \mathrm{P}$ NMR of $\mathbf{L} 7\left(162 \mathrm{MHZ}, \mathrm{CDCl}_{3}\right)$



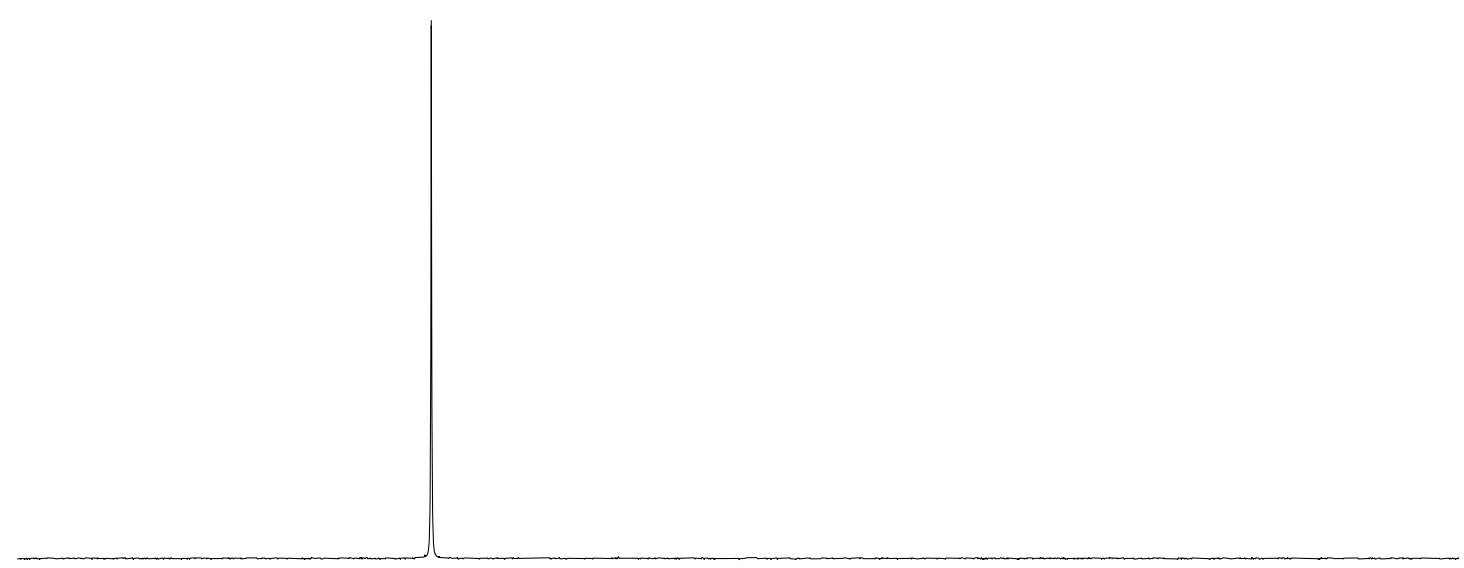

148146144142140138136134132130128126124122120118116114112110108106104102106 f1 (ppm) 